Linköping Studies in Science and Technology

Dissertations, No. 1772

\title{
Design of Energy-Efficient High-Performance ASIP-DSP Platforms
}

\author{
Andréas Karlsson
}

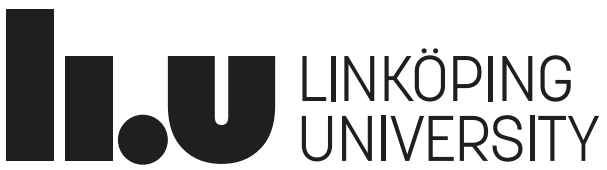

Department of Electrical Engineering

Linköping University

SE-581 83 Linköping, Sweden

Linköping 2016

ISBN 978-91-7685-745-8

ISSN 0345-7524 
Design of Energy-Efficient

High-Performance

ASIP-DSP Platforms

Andréas Karlsson

ISBN 978-91-7685-745-8

Copyright (C) Andréas Karlsson, 2016

Linköping Studies in Science and Technology

Dissertation No. 1772

ISSN 0345-7524

Department of Electrical Engineering

Linköping University

SE-581 83 Linköping

Sweden

Phone: +4613281000

Author e-mail: andreaskeisy.liu.se

\section{Cover image}

Chip layout of an ePUMA system with 8 compute clusters, each with a local controller, 8 compute cores and 1536kB of local compute data memory. The complete chip contains 73 processor cores and occupies $45 \mathrm{~mm}^{2}$ in 28nm FDSOI technology.

(Parts of this thesis are reprinted with permission from the IEEE.)

Printed by LiU-Tryck, Linköping University

Linköping, Sweden, 2016 


\section{Abstract}

In the last ten years, limited clock frequency scaling and increasing power density has shifted IC design focus towards parallelism, heterogeneity and energy efficiency. Improving energy efficiency is by no means simple and it calls for a reevaluation of old design choices in processor architecture, and perhaps more importantly, development of new programming methodologies that exploit the features of modern architectures.

This thesis discusses the design of energy-efficient digital signal processors with application-specific instructions sets, so-called ASIP-DSPs, and their programming tools. Target applications for such processors include, but are not limited to, communications, multimedia, image processing, intelligent vision and radar. These applications are often implemented by a limited set of kernel algorithms, whose performance and efficiency are critical to the application's success. At the same time, the extreme non-recurring engineering cost of system-on-chip designs means that product life-time must be kept as long as possible. Neither general-purpose processors nor non-programmable ASICs can meet both the flexibility and efficiency requirements, and ASIPs may instead be the best trade-off between all the conflicting goals.

Traditional superscalar- and VLIW processor design focus has been to improve the throughput of fine-grained instructions, which results in high flexibility, but also high energy consumption. SIMD architectures, on the other hand, are often restricted by inefficient data access. The result is architectures which spend more energy and/or time on supporting operations rather than actual computing. 
This thesis defines the performance limit of an architecture with an $\mathrm{N}$-way parallel datapath as consuming $2 \mathrm{~N}$ elements of compute data per clock cycle. To approach this performance, this work proposes coarsegrained higher-order functional (HOF) instructions, which encode the most frequently executed compute-, data access- and control sequences into single many-cycle instructions, to reduce the overheads of instruction delivery, while at the same time maintaining orthogonality. The work further investigates opportunities for operation fusion to improve computing performance, and proposes a flexible memory subsystem for conflict-free parallel memory access with permutation and lookup-tablebased addressing, to ensure that high computing throughput can be sustained even in the presence of irregular data access patterns. These concepts are extensively studied by implementing a large kernel algorithm library with typical DSP kernels, to prove their effectiveness and adequacy. Compared to contemporary VLIW DSP solutions, our solution can practically eliminate instruction fetching energy in many scenarios, significantly reduce control path switching, simplify the implementation of kernels and reduce code size, sometimes by as much as 30 times.

The techniques proposed in this thesis have been implemented in the DSP platform ePUMA (embedded Parallel DSP processor with Unique Memory Access), a configurable control-compute heterogeneous platform with distributed memory, optimized for low-power predictable DSP computing. Hardware evaluation has been done with FPGA prototypes. In addition, several VLSI layouts have been created for energy and area estimations. This includes smaller designs, as well as a large design with 73 cores, capable of 1280 integer GOPS or 256 GFLOPS at $500 \mathrm{MHz}$ and which measures $45 \mathrm{~mm}^{2}$ in 28nm FD-SOI technology.

In addition to the hardware design, this thesis also discusses parallel programming flow for distributed memory architectures and ePUMA application implementation. A DSP kernel programming language and its compiler is presented. This effectively demonstrates how kernels written in a high-level language can be translated into HOF instructions for very high processing efficiency. 


\section{Populärvetenskaplig sammanfattning}

Integrerade kretsar har idag en mycket central roll i vårt samhälle. De är en förutsättning för många moderna elektroniksystem och har revolutionerat vårt sätt att lära oss, arbeta, underhållas och kommunicera. I takt med att den integrerade kretsens minsta byggsten, transistorn, minskat i storlek, har det varit möjligt att konstruera mer kraftfulla system, där alltmer funktionalitet kan rymmas på en given kretsarea. Utöver detta har systemets arbetstakt, dess klockfrekvens, kunnat ökas, vilket direkt ger prestandavinster. De senaste tio åren har dock de positiva effekterna av fortsatt minskade kretskomponenter avtagit. Bidragande orsaker är de läckströmmar som uppstår när transistorerna görs mindre och att kretsarnas matningsspänning inte kan minskas i samma takt som tidigare. När nu den ensamma generella processorns prestandautveckling kraftigt avtagit har istället flerkärniga- och specialiserade processorer blivit normen och deras utveckling drivs med fokus på energieffektivitet. Detta får stora konsekvenser på hårdvaran, men även på hur moderna system ska programmeras.

Den här avhandlingen behandlar en speciell typ av processorer, digitala signalprocessorer (DSP), med applikationsspecifika instruktionsset. Applikationsspecifik innebär i detta sammanhang att processorn har utrustats med hårdvara anpassad för ett visst användningsområde, exempelvis trådlös kommunikation, digital ljud- och bildbehandling eller 
radar. Att processorn har specialanpassats är ofta en förutsättning för att dessa system ska kunna implementeras, p.g.a. de väldigt höga prestandaoch effektivitetskraven som ofta ställs. Däremot har de en del likheter, bl.a. att några få huvudalgoritmer körs väldigt frekvent. Dessa likheter som kan utnyttjas för att designa en processorplattform som kan rymma många applikationer, om viss anpassning kan göras.

I denna avhandling presenteras flera tekniker som kan minska energiförbrukningen i ett processorsystem. En huvuddel utgörs av att införa processorinstruktioner som utför väsentligt mycket mer arbete än traditionella finkorniga instruktioner, huvudsakligen för att minska totala strömförbrukningen från hämtning och avkodning av instruktioner. Den valda abstraktionsnivån för instruktionerna är noggrant vald för att passa digital signalbehandling och liknar funktionaliteten hos högre ordningens funktioner i funktionella språk. Utöver detta presenteras ett minnessystem vars syfte är att undvika de prestandaflaskhalsar som ofta uppstår på andra system med parallela beräkningselement. Att denna abstraktionsnivå är lämplig och tillräcklig utvärderas genom att implementera ett större antal DSP-algoritmer på demonstrationsplattformen ePUMA (embedded Parallel DSP processor with Unique Memory Access). Utvärderingen visar på vinster både i energieffektivitet och kodstorlek jämfört med andra samtida system.

Avhandlingen diskuterar även hur ovan nämnda processorsystem kan programmeras. Speciellt beskrivs designen av ett programmeringsspråk för DSP som samdesignats med hårdvaran för att med relativ enkelhet kunna utnyttja de föreslagna instruktionerna. Om vissa riktlinjer efterföljs i skriven högnivå-kod, kan koden i många fall enkelt översättas till slutgiltig maskinkod vars prestanda närmar sig plattformens teoretiska begränsning. 


\section{Preface}

This thesis includes material from the following first-author publications:

- Andréas Karlsson, Joar Sohl and Dake Liu.

Energy-efficient sorting with

the distributed memory architecture ePUMA.

In Proceedings of the International Symposium on Parallel and Distributed Processing with Applications (ISPA), 2015.

- Andréas Karlsson, Joar Sohl and Dake Liu.

Cost-efficient Mapping of 3- and 5-point DFTs to

General Baseband Processors.

In Proceedings of the International Conference on Digital Signal Processing (DSP), 2015.

- Andréas Karlsson, Joar Sohl and Dake Liu.

Software-based QPP Interleaving for

Baseband DSPs with LUT-accelerated Addressing.

In Proceedings of the International Conference on Digital Signal Processing (DSP), 2015.

- Andréas Karlsson, Joar Sohl and Dake Liu. ePUMA: A Processor Architecture for Future DSP.

In Proceedings of the International Conference on Digital Signal Processing (DSP), 2015. 
- Andréas Karlsson, Joar Sohl, Jian Wang and Dake Liu.

ePUMA: A unique memory access based

parallel DSP processor for SDR and CR.

In Proceedings of the Global Conference on Signal and Information Processing (GlobalSIP), 2013.

In addition, I have co-authored the following papers:

- Mehmet Ali Arslan, Flavius Gruian, Krzysztof Kuchcinski and Andréas Karlsson.

Code Generation for a SIMD Architecture

with Custom Memory Organization.

Accepted for presentation at Conference on Design and Architectures for Signal and Image Processing (DASIP), 2016.

- Essayas Gebrewahid, Mehmet Ali Arslan, Andréas Karlsson and Zain Ul-Abdin.

Support for Data Parallelism in the CAL Actor Language.

In 3rd Workshop on Programming Models for SIMD/Vector Processing, 2016.

- Joar Sohl, Andréas Karlsson and Dake Liu.

Conflict-free data access for

multi-bank memory architectures using padding.

In Proceedings of the International Conference on High Performance Computing (HiPC), 2013.

- Jian Wang, Andréas Karlsson, Joar Sohl and Dake Liu.

Convolutional decoding on deep-pipelined SIMD processor with flexible parallel memory.

In Proceedings of the Euromicro Conference on Digital System Design (DSD), 2012.

- Joar Sohl, Jian Wang, Andréas Karlsson and Dake Liu.

Automatic permutation for arbitrary static access patterns.

In Proceedings of the International Symposium on Parallel and Distributed Processing with Applications (ISPA), 2012. 
- Jian Wang, Andréas Karlsson, Joar Sohl, Magnus Pettersson and Dake Liu.

A multi-level arbitration and

topology-free streaming network for chip multiprocessor.

In Proceedings of the International Conference on ASIC (ASICON), 2011.

- Dake Liu, Andréas Karlsson, Joar Sohl, Jian Wang, Magnus Pettersson and Wenbiao Zhou.

ePUMA embedded parallel DSP processor with unique memory access.

In Proceedings of the International Conference on Information, Communications and Signal Processing (ICICS), 2011.

- Jian Wang, Joar Sohl, Andréas Karlsson and Dake Liu.

An efficient streaming star network for multi-core parallel DSP processor. In Proceedings of the International Conference on Networking and Computing (ICNC), 2011.

- Jens Ogniewski, Andréas Karlsson and Ingemar Ragnemalm.

Texture compression in memory and performance-constrained embedded systems.

In Proceedings of the IADIS International Conference Computer Graphics, Visualization, Computer Vision and Image Processing, 2011.

- Ingemar Ragnemalm and Andréas Karlsson.

Computing the Euclidian Distance Transform on

the ePUMA Parallel Hardware.

In Proceedings of the IADIS International Conference Computer Graphics, Visualization, Computer Vision and Image Processing, 2011. 


\section{Contributions}

The main contributions of this work can be summarized as:

- Analysis of DSP applications and current DSP processor designs: This thesis investigates the characteristics of typical DSP applications, information which is essential when designing efficient DSP processor architectures. Existing processor architectures, both from the research community and the commercial market, are analyzed and discussed, in order to find weak spots and suggest improvements. The goal is to see whether it is possible to merge applications such as baseband, multimedia, image processing and radar, under a common DSP platform and maintain high efficiency.

- Higher-order functional (HOF) instructions: HOF instructions are proposed as a way of improving energy efficiency, performance and reduce code size compared to contemporary DSP designs, by creating instructions that fuse large DSP computing subgraphs with data access and control. HOF instructions raises the level of abstraction of hardware instructions and bridges the semantic gap between hardware instructions and high-level languages.

- Analysis and design of DSP memory subsystems: The core-local memory requirements for DSP processor cores are thoroughly analyzed. A memory subsystem that supports penalty-free unaligned and out-of-order data access of vector data is presented as well as appropriate address generation circuitry. Lookup-table-based addressing is presented as a solution to support general addressing 
patterns. Special attention is also given to access of persistent data, specifically how to optimize the retrieval of program code, constant data and permutation vectors during DSP kernel execution.

- The ePUMA architecture: The ePUMA architecture is presented as a demonstration platform of a heterogeneous parallel multi-core DSP, with separate compute- and control processors. The ePUMA platform has been designed as a highly configurable platform, in order to be able to customize it to different application needs. The platform is analyzed and compared with other architectures.

- Design of compute-oriented DSP co-processors: The ePUMA architecture includes the ePUMA Matrix Processing Element (MPE), a DSP co-processor that implements the proposed HOF instructions and memory subsystem. This thesis presents its architecture in detail, discusses its design trade-offs and demonstrates why it may be a suitable architecture for compute-oriented DSP tasks.

- DSP kernel library: A large kernel library for the ePUMA MPE has been implemented and is used for MPE evaluation. The kernel library particularly demonstrates the effectiveness of HOF instructions and the flexible local memory subsystem.

- eOctave: The design of a domain-specific DSP programming language is presented: eOctave. The implementation demonstrates how high-level language constructs can be directly translated into HOF instructions and how highly efficient kernels can be implemented in a high-level language.

- System programming model: Programming models for heterogeneous scratchpad-based multi-core architectures are discussed. An appropriate programming model for the ePUMA demonstration system is presented. 


\section{Acknowledgments}

A lot of people have directly or indirectly been involved in the creation of this thesis. You all deserve my sincere thanks! I'd like to especially mention the following people:

- Prof. Dake Liu, for the supervision and opportunity to work in the ePUMA research project, and for your never-ending enthusiasm, encouragement and guidance.

- My fellow ePUMA Ph.D. student colleagues, Dr. Joar Sohl, Dr. Jian Wang and Magnus Pettersson, for all the work you've put in to the project, all interesting discussions and all the fun!

- Dr. Andreas Ehliar, for the co-supervision, all help and all discussions.

- Olof Kraigher, for introducing me to the ePUMA project as my master student supervisor and for your early contributions to the ePUMA project.

- Gunnel Hässler, Anita Kilander and Ylva Jernling, for all help with administrative issues.

- Anders Nilsson, for all help with software-related issues.

- Arta Alvandpour, for all help with EDA tools and CMOS memories.

- All ePUMA master thesis students, for all your valuable contributions to the project. 
- "Lunchlådemaffian", for all "truly important" discussions that enriched my everyday lunch experience.

- All staff at the Computer Engineering division, past and present, for all help, for being great colleagues and for making my working hours more enjoyable.

- My family and friends, for all your support and encouragement!

This research was supported by the Swedish Foundation for Strategic Research (SSF), projects DSP platform for emerging telecommunications and multimedia (RE07-0061) and HiPEC: High-Performance Embedded Computing (RE10-0081), and EU FP7-ICT project Multi-Base: Scalable Multitasking Baseband for Mobile Communications (216541).

Andréas Karlsson

Linköping, July 2016 


\section{Contents}

I Background 1

1 Introduction 3

1.1 Motivation .................... 5

1.2 Goals and scope . . . . . . . . . . . . . . 8

1.3 Thesis organization ............... 9

2 Processor architectures and energy efficiency 11

2.1 Energy consumption in static CMOS . . . . . . . . . . . 11

2.1.1 Interconnect- and off-chip memory access energy . 13

2.1.2 The end of Dennard scaling . . . . . . . . . . . . 14

2.2 Parallel processor architectures . . . . . . . . . . . 15

2.2.1 Superscalar and VLIW architectures . . . . . . . . 15

2.2.2 SIMD and vector architectures . . . . . . . . 18

2.2.3 Multi-core architectures ............. 19

2.3 Heterogeneity . . . . . . . . . . . . . . 20

2.3.1 Single-ISA multi-core heterogeneity . . . . . . . . 20

2.3.2 Multi-ISA multi-core heterogeneity . . . . . . . 20

2.3.3 Execution unit specialization . . . . . . . . . . 21

2.3.4 Full-custom platform heterogeneity . . . . . . . . 21

2.4 Memory hierarchy . . . . . . . . . . . . . 21

2.4.1 Shared memory and distributed memory . . . . . . 21

2.4.2 Memory coherency and memory consistency . . . . 24

2.4.3 Memory bandwidth . . . . . . . . . . . . 24

2.5 Energy reduction techniques ............ 25 
2.5.1 Voltage- and frequency scaling ......... . . . 26

2.5.2 Clock- and power-gating .......... 27

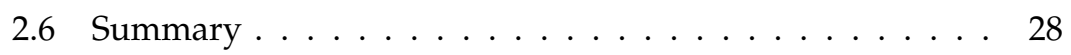

3 DSP application characteristics 29

3.1 Digital signal processing systems . . . . . . . . . . . . . . 29

3.2 Application examples . . . . . . . . . . . . . . 30

3.2.1 Software-defined radio . . . . . . . . . . 30

3.2.2 Media processing .............. 31

3.2 .3 Image processing . . . . . . . . . . . . 31

3.2 .4 Radar................... 32

3.3 Common characteristics ............. 32

3.3.1 Real-time constraints ............. 32

3.3.2 Vector/matrix operations ........... 32

3.3 .3 Data types ................ 32

3.3.4 Data access patterns ............ 33

3.3.5 Computing kernels . . . . . . . . . . . . 33

3.3 .6 Predictability ................... 34

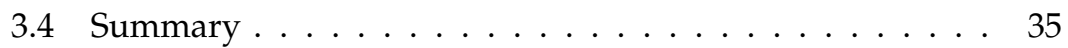

4 Research methodology $\quad 37$

4.1 General methodology . . . . . . . . . . . . . . . 37

4.2 Kernel-based hardware/software co-design . . . . . . . . 38

4.3 Retrospective ................ 40

II The ePUMA architecture 41

5 The ePUMA architecture 43

5.1 Design space exploration . . . . . . . . . . . . 43

5.1.1 Compute/control separation .......... . 44

5.1 .2 Memory subsystem . . . . . . . . . . . . 45

5.1 .3 Summary of architecture goals . . . . . . . . . 47

5.2 The ePUMA hardware architecture . . . . . . . . . . . . 48

5.2.1 The compute cluster . . . . . . . . . . . . 49 
5.2 .2 The memory hierarchy . . . . . . . . . . . 51

5.2 .3 The network-on-chip . . . . . . . . . . 52

5.3 System configurability .............. 53

5.4 Programming .................. 54

5.5 Application execution ................ 56

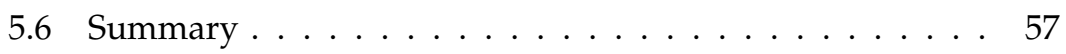

6 Related work $\quad 59$

6.1 Processor generators ................ 59

6.2 Texas Instruments DSPs . . . . . . . . . . . . . . 60

6.3 CEVA DSPs . . . . . . . . . . . . . . 61

$6.4 \mathrm{NXP} /$ Freescale DSPs . . . . . . . . . . . . 62

6.5 Coresonic LeoCore . . . . . . . . . . . . . . . . . . . 62

6.6 SODA and Ardbeg ................. 64

6.7 Movidius Myriad $2 \ldots . . \ldots 65$

6.8 The ELM architecture . . . . . . . . . . . . . . 66

6.9 The MediaBreeze architecture . . . . . . . . . . . . . 66

6.10 STI Cell Broadband Engine . . . . . . . . . . . . . . 66

6.11 x86, ARM and PowerPC . . . . . . . . . . . . 67

6.12 Other platforms . . . . . . . . . . . . . . 68

6.13 Summary ...................... 68

\section{Design of compute-optimized DSP co-processors 69}

7 Higher-order functional instructions $\quad 71$

7.1 Motivation . . . . . . . . . . . . . 72

7.2 Goals ......................... 73

7.3 Computing patterns ... . . . . . . . . 73

7.3.1 The map, reduce and map-reduce patterns . . . . . . . 74

7.3 .2 Block patterns . . . . . . . . . . . 75

7.4 Data access . . . . . . . . . . . . . 78

7.5 The higher-order functional instruction . . . . . . . . . 79

7.6 Kernel examples . . . . . . . . . . . . . . . . . . . 81

7.6.1 Element-wise operations . . . . . . . . . . . 81 
7.6.2 Convolution and FIR filtering . . . . . . . . 82

7.6.3 Fast Fourier Transform . . . . . . . . . . . . 83

7.7 Related instruction sets and architectures . . . . . . . . 84

7.7.1 VLIW and superscalar architectures . . . . . . . 84

7.7.2 SIMD and vector architectures . . . . . . . 85

7.7.3 Application-specific architectures . . . . . . . 87

7.7.4 Comparative summary . . . . . . . . . 88

7.8 Summary . . . . . . . . . . . . . . . . 91

8 Local memory architecture $\quad 93$

8.1 Memory subsystem requirements . . . . . . . . . . . 93

8.2 Multi-bank memories . . . . . . . . . . . . . . 96

8.2.1 Terminology .................. 96

8.2.2 Memory bank granularity . . . . . . . . . 98

8.2 .3 Memory width . . . . . . . . . . . 100

8.2.4 Memory size................ 103

8.3 Conflict-free parallel memory access . . . . . . . . . . . 103

8.3.1 Permutation ................. 105

8.3.2 Padding ................... 107

8.3.3 Multiple buffering . . . . . . . . . . 108

8.3 .4 Scalability .................. 110

8.4 Kernels and memory parallelism . . . . . . . . . . . . 112

8.4.1 Kernels with constant data . . . . . . . . . . 115

8.4.2 Bandwidth requirements for reduction patterns . . 115

8.4.3 Bandwidth requirements for map patterns . . . . 117

8.4.4 System-level considerations . . . . . . . . . . 118

8.5 Address generation . . . . . . . . . . . . . . . . . . 119

8.5.1 Specification by example . . . . . . . . . 120

8.5 .2 AGU features . . . . . . . . . . . . 126

8.6 Conflict-free unpredictable memory access . . . . . . . . 128

8.7 Related work . . . . . . . . . . . . . . . 133

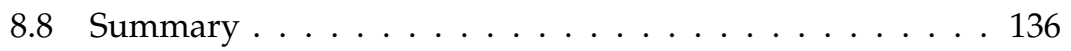


9 Handling the complexities of storage and access of persistent $\begin{array}{ll}\text { data } & 137\end{array}$

9.1 Problem definition ... . . . . . . . . . . 138

9.2 Motivation and goals . . . . . . . . . . . . . . 139

9.3 Related work . . . . . . . . . . . . . . . . 139

9.4 Kernel analysis . . . . . . . . . . . . . . . . . . . 141

9.4.1 Permutation table access . . . . . . . . . . . 144

9.4.2 Constant data usage behavior . . . . . . . . . . 145

9.5 Explicit data management and cache-based solutions . . . 146

9.6 Multi-bank memory . . . . . . . . . . . . . . 148

9.7 Pipeline organization . . . . . . . . . . . . . 150

9.8 Evaluation ..................... 152

9.9 Summary ........................ 156

10 Datapath design and optimization 157

10.1 Datapath requirements . . . . . . . . . . . . . 158

10.2 Designing an application-specific datapath . . . . . . . . 159

10.3 Datapath pipeline depth considerations . . . . . . . . . . 162

10.4 Optimizing arithmetic unit networks . . . . . . . . . . 165

10.4.1 General problem formulation . . . . . . . . . . . 167

10.4.2 General constraints for operation mapping . . . . . 171

10.4.3 Mapping instructions to the datapath . . . . . . 172

10.4.4 Optional constraints . . . . . . . . . . . 175

10.4 .5 Limitations . . . . . . . . . . . . 176

10.4.6 Evaluation . . . . . . . . . . . . . 176

10.4.7 Summary . . . . . . . . . . . . . . 179

10.5 Datapath reconfigurability . . . . . . . . . . . . 180

10.6 Summary . . . . . . . . . . . . . . . . . 182

11 The ePUMA Matrix Processing Element 183

11.1 Goals ...................... 183

11.2 The MPE architecture . . . . . . . . . . . . . . . . . . . 184

11.3 Physical characteristics . . . . . . . . . . . . . . 187

11.4 Pipeline scheduling . . . . . . . . . . . . . . . . 189 
11.5 Memory consistency . . . . . . . . . . . . . . . . . . . . . 189

11.6 Reducing performance unpredictability . . . . . . . . . . 191

11.6.1 Vector length independent execution and iteration capability . . . . . . . . . . . . . . . 191

11.6.2 Control flow removal . . . . . . . . . . . . . . 192

11.6.3 Predicated execution and control flow . . . . . . . 192

11.7 Register file addressing . . . . . . . . . . . . . . . . . 193

11.8 Element-based hardware repeat . . . . . . . . . . . . . . 194

11.9 MPE control . . . . . . . . . . . . . . . . . . . . . . . . 199

11.10 Summary . . . . . . . . . . . . . . . . . . . . . 201

IV System configuration and programming 203

12 Platform generation $\quad 205$

12.1 Usage scenarios . . . . . . . . . . . . . . . . . . . . 205

12.1.1 Massively parallel processing . . . . . . . . . . . 206

12.1.2 Dataflow DSP . . . . . . . . . . . . . . . . . . . . 206

12.1.3 Application-specific DSP . . . . . . . . . . . . . 207

12.1.4 General DSP . . . . . . . . . . . . . . . . . . 207

12.2 Architecture selection . . . . . . . . . . . . . . . . . . 208

12.3 Platform creation process . . . . . . . . . . . . . . 209

12.4 Summary . . . . . . . . . . . . . . . . . . . . . 211

13 Kernel programming 213

13.1 Goals . . . . . . . . . . . . . . . . . . . . . . . . . . 213

13.2 Kernel programming flow . . . . . . . . . . . . . . . . . . . . . . . 214

13.3 Related work . . . . . . . . . . . . . . . . . . . . . . . . . 215

13.4 The eOctave kernel programming language . . . . . . . 216

13.4.1 Motivation . . . . . . . . . . . . . . . . . . . 216

13.4.2 Design goals . . . . . . . . . . . . . . . . . 217

13.4.3 Design methdology . . . . . . . . . . . . . . . . 218

13.4.4 eOctave kernel example . . . . . . . . . . . . . . . 219

13.4.5 Compiler implementation . . . . . . . . . . . . 224

13.4.6 MPE kernel library implementation . . . . . . . 224 
13.5 Summary . . . . . . . . . . . . . . . . 225

14 System programming $\quad 227$

14.1 Parallel programming frameworks . . . . . . . . . . . . . 227

14.2 Kernel-based programming . . . . . . . . . . . . . 230

14.3 ePUMA programming flow . . . . . . . . . . . . 231

14.4 Programming tools . . . . . . . . . . . . . . . . 231

14.5 Operating system support . . . . . . . . . . . . . . 232

14.6 ePUMA application example . . . . . . . . . . . . . . 233

14.7 Summary . . . . . . . . . . . . . . . 233

$\begin{array}{llr}\text { V Evaluation } & 237\end{array}$

15 MPE evaluation $\quad 239$

15.1 Methodology . . . . . . . . . . . . . 239

15.2 Case study: Basic vector/matrix element-wise operations . 244

15.3 Case study: Reductions . . . . . . . . . . . . . . . . . 246

15.4 Case study: Data rearrangement . . . . . . . . . . . . . . 247

15.4.1 QPP interleaving . . . . . . . . . . . . . 249

15.5 Case study: Linear filters . . . . . . . . . . . . . . . . 251

15.5.1 Implementation of a general FIR-filter . . . . . . . . 251

15.5.2 Comparison with an FIR-filtering ASIC . . . . . . 256

15.5 .3 2D convolution . . . . . . . . . . . . 258

15.6 Case study: Discrete Fourier Transform . . . . . . . . . . . 261

15.6.1 Non-power-of-2 transforms . . . . . . . . . . 262

15.7 Case study: Histogram generation . . . . . . . . . . . . . 265

15.8 Case study: Sorting and non-linear filters . . . . . . . . . 268

15.8.1 Median filtering . . . . . . . . . . . . 273

15.9 General evaluation . . . . . . . . . . . . . . 275

15.10Alternative MPE versions . . . . . . . . . . . . . 278

15.10.1 Increasing processing width . . . . . . . . . . . 279

15.10.2 Improved floating-point performance . . . . . . . . 281

15.10.3 Advanced floating-point MPE . . . . . . . . . . 282

15.11Summary .................... 283 
16 System evaluation $\quad 285$

16.1 Sorting on ePUMA . . . . . . . . . . . . . . 285

16.1.1 Multi-way merging . . . . . . . . . . . . . 289

16.1.2 Evaluation . . . . . . . . . . . . . . 290

16.2 Other algorithms and applications . . . . . . . . . . . 293

16.3 System hardware evaluation . . . . . . . . . . . . . . . 294

16.4 Summary . . . . . . . . . . . . . . . . . . 294

VI Conclusions and future work 297

17 Conclusions $\quad 299$

17.1 Computing- and energy efficiency . . . . . . . . . . . 300

17.2 Predictability . . . . . . . . . . . . . . . 300

17.3 Final remarks . . . . . . . . . . . . . . . . . . . . 301

18 Future work $\quad 303$

18.1 Hardware aspects . . . . . . . . . . . . . . . 303

18.2 System tool-chain . . . . . . . . . . . . . . . . 304

18.3 Application development . . . . . . . . . . . . . 305

18.4 Release of ePUMA . . . . . . . . . . . . 305

$\begin{array}{ll}\text { References } & 307\end{array}$

$\begin{array}{lll}\text { VII Appendix } & 327\end{array}$

A ePUMA hardware/software components 329

B ePUMA development timeline 333

B.1 ePUMA version $1.0 \ldots \ldots 33$

B.2 ePUMA version $1.5 \ldots \ldots \ldots$. . . . . . . . . . . 334

B.3 ePUMA version $2.0 \ldots \ldots$. . . . . . . . . . . . . 334

B.4 ePUMA version $3.0 \ldots \ldots$. . . . . . . . . . . . 335

C MPE kernel library $\quad 337$ 


\section{Abbreviations}

ACC

ADC

AGU

ALU

API

ASIC

ASIP

AU

CC

$\mathrm{CCC}$

CISC

CMOS

CMP

CPU

CUDA

DAC

DCT

DFT

DLP

DMA

DRAM

DSP

DVFS

EDA
Accelerator

Analog-to-Digital Converter

Address Generation Unit

Arithmetic and Logic Unit

Application Programming Interface

Application-Specific Integrated Circuit

Application-Specific Instruction Set Processor

Arithmetic Unit

Compute Cluster

Compute Cluster Controller

Complex Instruction Set Computing

Complementary Metal Oxide Semiconductor

Chip-Multiprocessor

Central Processing Unit

Compute Unified Device Architecture

Digital-to-Analog Converter

Discrete Cosine Transform

Discrete Fourier Transform

Data Level Parallelism

Direct Memory Access

Dynamic Random-Access Memory

Digital Signal Processing or Digital Signal Processor

Dynamic Voltage and Frequency Scaling

Electronic Design Automation 


\begin{tabular}{|c|c|}
\hline FD-SOI & Fully-Depleted Silicon-On-Insulator \\
\hline FEC & Forward Error Correction \\
\hline FFT & Fast Fourier Transform \\
\hline FIFO & First In First Out \\
\hline FIR & Finite Impulse Response \\
\hline FPGA & Field-Programmable Gate Array \\
\hline FPU & Floating-Point Unit \\
\hline FIR & Finite Impulse Response \\
\hline GFLOPS & Giga-Floating-point Operations per Second \\
\hline GIOPS & Giga-Integer Operations per Second \\
\hline GOPS & Giga-Operations per Second \\
\hline GPP & General Purpose Processor \\
\hline GPU & Graphics Processing Unit \\
\hline HEVC & High Efficiency Video Coding (H.265) \\
\hline HOF & Higher-Order Function/Functional \\
\hline IDCT & Inverse Discrete Cosine Transform \\
\hline IDFT & Inverse Discrete Fourier Transform \\
\hline IFFT & Inverse Fast Fourier Transform \\
\hline IIR & Infinite Impulse Response \\
\hline ILP & Instruction-Level Parallelism or Integer Linear Programming \\
\hline IMC & Integrated Memory Controller \\
\hline IP & Intellectual Property \\
\hline IPC & Instructions per Cycle \\
\hline IR & Intermediate Representation \\
\hline ISA & Instruction Set Architecture \\
\hline ITRS & International Technology Roadmap for Semiconductors \\
\hline JPEG & Joint Photographic Experts Group \\
\hline LS & Local Store \\
\hline LSU & Load-Store Unit \\
\hline LTE & Long Term Evolution \\
\hline LU & Logic Unit \\
\hline LUD & LU Decomposition \\
\hline LUT & Lookup Table \\
\hline
\end{tabular}




$\begin{array}{ll}\text { LVM } & \text { Local Vector Memory } \\ \text { MAC } & \text { Multiply and Accumulate } \\ \text { MCU } & \text { Microcontroller Unit } \\ \text { MFC } & \text { Memory Flow Controller } \\ \text { MM } & \text { Main Memory } \\ \text { MMU } & \text { Memory Management Unit } \\ \text { MPE } & \text { Matrix Processing Element } \\ \text { MPEG } & \text { Moving Picture Experts Group } \\ \text { MPEG-4 AVC } & \text { MPEG-4 Advanced Video Coding (H.264) } \\ \text { NI } & \text { Network Interface } \\ \text { NoC } & \text { Network on Chip } \\ \text { NOP } & \text { No Operation } \\ \text { OS } & \text { Operating System } \\ \text { PCB } & \text { Printed Circuit Board } \\ \text { PHY } & \text { Physical } \\ \text { PMU } & \text { Power Management Unit } \\ \text { PPU } & \text { Packet Processing Unit } \\ \text { PM } & \text { Program Memory } \\ \text { PT } & \text { Permutation Table } \\ \text { QRD } & \text { QR Decomposition } \\ \text { RISC } & \text { Reduced Instruction Set Computing } \\ \text { RTL } & \text { Register Transfer Level } \\ \text { SAGU } & \text { Scalar Address Generation Unit } \\ \text { SDR } & \text { Software Defined Radio } \\ \text { SFG } & \text { Signal Flow Graph } \\ \text { SIMD } & \text { Single Instruction Multiple Data } \\ \text { SPMD } & \text { Single Program Multiple Data } \\ \text { SoC } & \text { System on a Chip } \\ \text { SVD } & \text { Singular Value Decomposition } \\ \text { TDP } & \text { Thermal Design Power } \\ \text { TLP } & \text { Thread-Level Parallelism } \\ \text { UAV } & \text { Unmanned Aerial Vehicle } \\ \text { VAGU } & \text { Vector Address Generation Unit } \\ & \end{array}$


VLIW Very Long Instruction Word

VLSI Very-Large-Scale Integration 


\section{Symbols}

$\alpha \quad$ Logical memory address

$B \quad$ The number of memory banks of a memory

$b \quad$ Physical memory bank index

$\beta \quad$ Physical memory address within a given memory bank

$L_{\text {pipe }} \quad$ Pipeline latency / length

$L_{\text {setup }} \quad$ Setup latency

$L_{w r} \quad$ Write-to-read latency

$L_{w r, r f} \quad$ Register file write-to-read latency

$L_{w r, m e m} \quad$ Memory write-to-read latency

$M \quad$ Total size of a memory

$M_{b} \quad$ Total size of a memory bank

$N \quad$ Total number of operations

$N_{i} \quad$ Number of operations in inner HOF dimension

$N_{o} \quad$ Number of operations in outer HOF dimension

$\nu \quad$ Natural processing width

$\nu_{\text {lane }} \quad$ Number of bits per processing lane

$\nu_{b} \quad$ Width of memory bank

$P \quad$ SIMD processing width / number of processing lanes

$P_{d p} \quad$ Number of datapaths

$P_{o p} \quad$ Effective operand bandwidth

$P_{\text {ports }} \quad$ Number of ports to multi-ported memory

$\Psi_{b} \quad$ Bank assignment function

$\Psi_{\beta} \quad$ Address assignment function 


\section{Part I}

\section{Background}





\section{Chapter 1}

\section{Introduction}

Modern society has rapidly become very much dependent on high performance digital signal processing technologies, a process which has been possible due to the rapid evolution of integrated circuit technology. It's a key enabler of technologies such as wireless communications, image-, audio-, speech- and movie processing, sensor array processing, biomedical signal processing and many other applications. High-speed wireless Internet access is available in very small devices, but the demand for even higher performance is very strong. New technologies such as autonomous cars and devices implanted in the human body may further increase performance and energy efficiency requirements. It is clear that we haven't seen the end of the demand for even higher system performance and there is clearly much to be done in in the area of signal processing, to be able to cope with the applications of tomorrow.

For roughly 50 years, digital system designers have enjoyed the device scaling predicted by Moore's law [1]. It has allowed an exponential growth of the number of transistors that can fit on a given chip area, and, in combination with increased clock frequencies, we have seen exponential growth of digital system performance. However, since the breakdown of Dennard scaling [2] in around 2005, a chip's power density is no longer constant with device scaling and is instead increasing. With commercial CPUs consuming in excess of 100 Watts of power, it is clear that the design focus had to shift from performance to performance per 
Watt, and consequently energy efficiency has become the new norm. It is especially important for battery-powered devices, where high power consumption results in unacceptable battery longevity.

Many of the processor circuit techniques that are used today were developed in the days when performance was the design norm and those techniques may not always represent the most energy-efficient choice. Several techniques have been mined out, such as instruction-level parallelism (ILP), and further increasing clock frequency is no longer feasible. The International Technology Roadmap for Semiconductors (ITRS) projects that the number of cores and system heterogeneity must scale aggressively to deliver the performance and energy efficiency required by the market [3]. Power management must be considered through the whole design process of a system-on-chip system, including system architecture and process technology, but also application software and programming methodology. In many product categories, the traditional $\mathrm{CPU}$ core is no longer central to product value and the interest has instead shifted towards graphics processing units (GPUs), digital signal processors (DSPs), field-programmable gate arrays (FPGAs) and accelerators. To unlock the potential of these massively parallel and heterogeneous systems, there is a strong need for improved parallel programming models and tools.

Figure 1.1 shows the trade-off between flexibility, performance and energy efficiency for different types of processing architectures, including general purpose processors (GPPs), general purpose DSPs (GP-DSPs), application-specific instruction set DSPs (ASIP-DSPs) and applicationspecific integrated circuits (ASICs). An ASIP-DSP can easily be more than two orders of magnitude more energy-efficient than a general purpose processor for appropriate processing tasks, due to specialization $[4,5,6]$. This is achieved through the use of more energy-efficient process technology, lower clock frequencies, less general instructions sets and application-specific instruction set additions and memory hierarchies to name a few examples. The cost of this specialization is less flexibility and typically also an increased programming effort. Still, it is a much more 


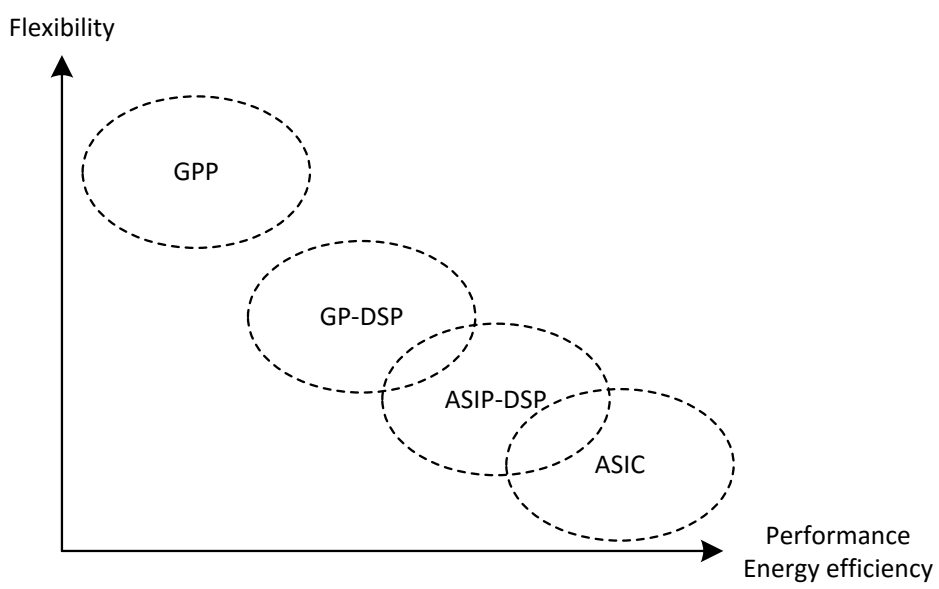

Figure 1.1: Comparison of signal processing architectures.

attractive solution than fixed-function ASICs, especially in multi-mode environments. An ASIP-DSP may be the best trade-off when we require moderate flexibility and energy efficiency that can come close to ASICs. Such DSPs is the main topic of this thesis.

\subsection{Motivation}

The specifications for a modern signal processing solution are full of conflicting requirements, which makes it non-trivial to design the perfect system. Typical requirements include:

- High performance: Many signal processing applications require very high compute performance. Off-the-shelf components may not provide the necessary performance within a given energy budget and one may be forced to specialize the architecture for its application domain.

- Low power consumption: Embedded systems often have limited cooling capabilities and run on battery. Low power operation is 
essential to deliver an acceptable level of product quality.

- High flexibility: The system must be able to be programmed, so that software upgrades and bug fixes can be applied. In multimode execution environments, it is beneficial if the processor can be used for many purposes to reduce overall cost.

- Long product lifetime: The lifetime of a signal processing product may be long and many products may need some room for future expansion.

- Low hardware cost: Many signal processing systems are high volume products on highly competitive markets. Low hardware cost per unit is essential to be able to deliver cheap products and still maintain some profit.

- Short time to market: A full system implementation, including both the hardware- and software design, must be possible to complete quickly, to stay ahead of competing companies.

A general-purpose of-the-shelf DSP processor may sometimes be an appropriate choice, since much of the hardware design phase is already done and the total product development time and cost may be reduced. However, if higher performance or lower power consumption is required, one may be forced to implement an application-specific platform. An ASIC solution may not be suitable, because of long development time and low flexibility. An ASIP-DSP represents a reasonable trade-off between all the requirements. Even though the development cost and implementation time of an application-specific processor can possibly be amortized over multiple products, its implementation time is still long, if done from scratch.

Instead of creating an application-specific processor from scratch, one can save a significant amount of time if one can start the design from some suitable base architecture and simply configure it for its specific use. Possible configurable parameters can include the selection of memory hierarchy, the number of processor cores and a set of application- 
specific instructions. To make the base architecture useful for many different applications, it is important that it is general enough to support them, but the generality should not come at too high a hardware cost. As we shall see, many DSP applications share similar features, which makes an excellent case for hardware reduction. By generating an applicationspecific platform from a general base architecture instead of starting from scratch, we get several benefits:

- Development time: The hardware development time will be significantly reduced.

- Application unknowns: The requirements of the application are possibly only partially known at hardware design time and it may be easier to do late modifications.

- Application uncertainty: Future software upgrades could require more hardware functionality for efficient execution. A more general architecture has a better chance of adapting to future needs.

- Design space exploration: It is much simpler to compare alternative architecture implementations.

- Hardware multiplexing: A more general architecture could possibly replace multiple very specific processors, to reduce hardware cost.

- Fewer hardware redesigns: If improved products are frequently released, it may be a better choice to keep generality to avoid costly hardware redesigns and verification.

- Code reuse: Critical DSP code is less likely to become obsolete and can be reused over time and products more easily.

- DSP kernel library: A base architecture can be shipped with a library of pre-implemented DSP algorithms of high quality, which helps to reduce software development time. 
- Shared tool-chain: Only a single platform tool-chain would have to be developed. The tool-chain would simply be configured with the parameters of the specific platform instance.

- Programmer experience: A programmer's experience with one platform instance is still very useful if he/she moves to work with another platform instance, since the programming methodology and tool-chain would be similar.

By designing an application-specific platform from a base architecture, we clearly gain many advantages. It's also the main motivation to why it is important to find such an architecture.

\subsection{Goals and scope}

The main goal of the presented research is to derive a flexible DSP platform that can quickly be adapted to many different signal processing applications. The research includes both the hardware architecture, as well as the programming tools necessary for efficient application implementation. To make the base architecture as flexible as possible, one of the main goals is to identify similarities between different applications, so that all platforms derived from our base architecture share some common features. A platform is then made application-specific by selecting appropriate implementation parameters and by adding applicationspecific instructions and/or accelerators to the design.

The research includes the development of a new type of hardware instruction paradigm, that reduces the energy consumption from instruction delivery and control operations and makes sure that the execution units are fully utilized. Local data access is also addressed, to find solutions for conflict-free parallel data access in DSP processors. To prove the efficiency of the proposed solutions, an extensive set of DSP algorithm kernels have been implemented, which are then compared with alternative architectures.

The research has led to the demonstration platform ePUMA (embed- 
ded Parallel DSP platform with Unique Memory Access), a collective effort by several people. The system-level design and network-on-chip is presented in [7]. Software-related aspects are detailed in [8]. This thesis primarily focuses on the processor core-level design and core-level toolchain issues. The work distribution in relation to the produced hardware components and software tools is summarized in Appendix A. ePUMA exists in several versions, which represent various stages of development and refinement of the ePUMA platform. These versions are summarized in Appendix B.

As this thesis primarily focus on application-specific DSPs, all ideas may not be fully applicable to other types of platforms, since additional constraints could prohibit the use of some of the proposed techniques. At the same time, the goal of this thesis is not to discuss any particular application in too much detail. Such research has been done at our division earlier $[9,10]$ and the great success of that research is one of the main sources of inspiration for this work.

\subsection{Thesis organization}

This thesis have been divided into the following parts:

Part I includes this introduction, as well as a review of processor design techniques and an analysis of the characteristics of DSP applications. In addition, the research methodology used in this work will be outlined.

Part II presents an overview of our demonstration platform ePUMA, the ePUMA Compute Cluster and a comparison with related contemporary architectures.

Part III presents the main hardware research, the work on the ePUMA Matrix Processing Element (MPE). It presents higher-order functional (HOF) instructions, design of local memory subsystems for DSPs and the MPE datapath design.

Part IV focuses on platform configuration and software aspects, such as the programming model for heterogeneous multi-core DSPs and especially the tool-chain for our ePUMA platform. The domain-specific 
language eOctave is presented, a DSP kernel programming language, codesigned with the ePUMA MPE processor to be able to effectively utilize its unique features.

Part V evaluates the presented research, both at the core-level and systemlevel.

Part VI presents the final conclusions and ideas about future work on the platform and related subjects.

This thesis also includes an appendix section, which lists all ePUMA hardware and software tools (Appendix A), the different versions of the ePUMA platform (Appendix B) and the complete list of implemented MPE algorithm kernels (Appendix C). Additional information about the ePUMA project can be found in the ePUMA manuals $[11,12,13]$. 


\section{Chapter 2}

\section{Processor architectures and energy efficiency}

This chapter will review the technology used to implement the processor architectures of today. Special attention will be given to reviewing alternative implementations from an energy efficiency perspective. To identify the sources of energy consumption in processors, we start by reviewing energy consumption in complementary metal oxide semiconductor (CMOS) circuits.

\subsection{Energy consumption in static CMOS}

A schematic of an inverter gate in CMOS technology is shown in Figure 2.1. The power consumption of a CMOS circuit can be modeled as

$$
\begin{aligned}
P_{\text {total }} & =P_{\text {dynamic }}+P_{\text {short-circuit }}+P_{\text {leakage }} \\
& =\left(C_{L} V_{D D}^{2}+V_{D D} I_{s c} t_{s}\right) f_{0 \rightarrow 1}+V_{D D} I_{\text {leak }}
\end{aligned}
$$

where $C_{L}$ is the switched capacitive load, $V_{D D}$ is the supply voltage, $I_{s c}$ is the peak short circuit current during gate switching, $t_{s}$ is the short circuit time, $f_{0 \rightarrow 1}$ is the equivalent number of gate output 0 to 1 transitions per second and $I_{\text {leak }}$ is the overall static leakage current [14]. If one instead is interested in the energy consumed over the execution of an al- 


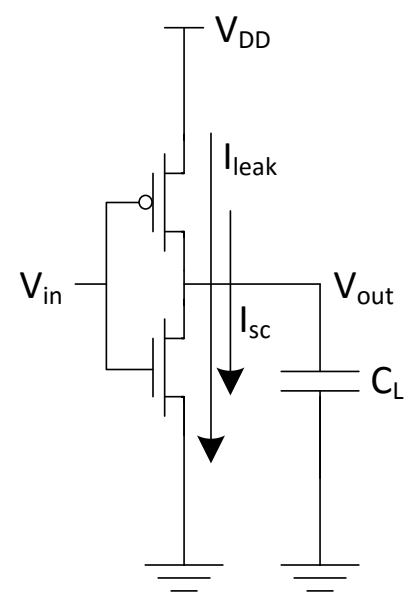

Figure 2.1: An inverter in CMOS technology.

gorithm or application, one can instead derive the following expression,

$$
E_{\text {total }}=\left(C_{L} V_{D D}^{2}+V_{D D} I_{s c} t_{s}\right) N_{0 \rightarrow 1}+V_{D D} I_{\text {leak }} t_{e x}
$$

where $N_{0 \rightarrow 1}$ is the number of times the total capacitive load $C_{L}$ is switched and $t_{e x}$ is the total execution time.

From the energy equation above, one can clearly see that effective approaches to reduce energy consumption would be to reduce the number of logic transitions and to reduce the supply voltage. Static energy consumption can be decreased by reducing the execution time. The energy consumption in integrated circuits have traditionally been dominated by dynamic energy consumption, but this is no longer a valid assumption. An additional problem is the parameters cannot be changed independently. Typically one cannot reduce the supply voltage without decreasing clock frequency, and thus performance, which would increase execution time. The target clock frequency of a design will highly influence several of these parameters, where a higher clock frequency target typically results in larger hardware area and therefore also higher dynamic and static energy consumption.

Device scaling has had a huge impact on energy efficiency for several 
decades. As transistors have been made smaller, the supply voltage and switched capacitance per gate have both decreased. This has made it possible to fit more functionality in the integrated circuit and increase performance without an exponential increase in power consumption. Unfortunately, the supply voltage in modern technologies is getting very close to the threshold voltage of the transistors. The roughly constant power density of integrated circuits predicted by Dennard [2] is therefore no longer a reality, since supply voltage reduction has slowed down [15].

The main goal of energy efficient processor design is to reduce the amount of energy consumed to execute a given task under some performance constraints. This is not the same as operating with a low power consumption, since low power consumption easily can be obtained by lowering performance, e.g. by lowering clock frequency and voltage. At the same time there is always some upper limit on allowed power consumption that must be met, due to practical limitations on a system's cooling capability. By keeping the performance high we may reduce the static energy consumed to run a particular algorithm, but if this is achieved through an excessive clock frequency, dynamic power consumption will be an issue. Instead, running at a moderate clock frequency, doing more work per clock cycle and reducing unnecessary logic switching may be the best approaches to reduce energy consumption.

\subsubsection{Interconnect- and off-chip memory access energy}

One aspect that is not immediately visible from the traditional energy consumption equations is interconnect. The problem with interconnect scaling is that local interconnect capacitance decreases as wires become smaller, but resistance per unit length increases. In practice this means that wires have not scaled as aggressively as transistors. It turns out that moving data around inside an integrated circuit, and moving data up and down in the memory hierarchy of a processor, can be a lot more energy consuming than actual compute operations. In addition, off-chip memory accesses are very expensive, and in some designs, such as in 
GPUs, off-chip accesses contribute with up to $20 \%$ of the whole GPUs power consumption [16].

Currently, it seems like transistor scaling may continue for at least 5-10 years and the current scaling trend suggests that data movement will be the dominating contributor to power consumption in future systems [17]. Data movement can be reduced at the architectural level, but using algorithms that require less data movement is equally important. Because of this, both hardware- and software designers must think of spatial locality, not only for performance reasons, but also for energy efficiency.

\subsubsection{The end of Dennard scaling}

As mentioned previously, a chip's power density can no longer be considered constant with scaling. This has some severe consequences for integrated circuit design. Transistors are still getting smaller, which makes it possible to put more transistors on a given chip area. However, the overall circuit activity must decrease in order to keep total power consumption constant. This issue has become known as the Dark Silicon problem $[18,19]$.

If not all transistors of a processor design can be used at the same time, we have to spend chip area in a way that minimizes switching, but still increases performance and energy efficiency. The size of memories can be increased, since memory is mostly a dark resource. Hardware multiplexing may be less useful, since specialized functional units are more energy efficient than more general ones, and the unrelated functional units could be turned off. Accelerators and specialized processor cores is another way forward. In practice all of these techniques have to be considered to design energy efficient systems. Many techniques will also directly impact software development. It is crucial that the programming models, programming tools and software programmers can adapt to these new design constraints, so that future systems can be successful. 


\subsection{Parallel processor architectures}

One of the best techniques to improve performance and energy efficiency is the exploit different forms of parallelism. Parallelism has been exploited for several decades in architectures such as superscalar processors and vector processors. Many of these techniques are primarily designed to increase performance, but they also improve energy efficiency, since the main alternative would be to increase clock frequency, which is not energy efficient or even practical. There are mainly three types of parallelism exploited in processor architectures:

- Instruction-level parallelism (ILP): Data dependencies between instructions in the instruction stream are evaluated at compile-time or run-time, to determine which instructions potentially could be executed in parallel over multiple execution units.

- Data-level parallelism (DLP): The same operation is performed on multiple data elements in parallel.

- Thread-level parallelism (TLP): Multiple instructions from multiple threads are executed in parallel on the hardware.

In practice, all three forms of parallelism are often supported. The following three subsections will survey the most common approaches to exploit these forms of parallelism.

\subsubsection{Superscalar and VLIW architectures}

The two most prominent examples of architectures exploiting ILP are the superscalar and Very Long Instruction Word (VLIW) architectures. The main difference between these two architectures is whether dependency checking between operations is done at run-time or compile-time.

Superscalar processors implement instruction-level parallelism by letting hardware logic inspect the sequential stream of instructions in order to find data dependencies, and issue independent instructions in parallel over multiple execution units [20]. The principle is shown in Figure 2.2. 


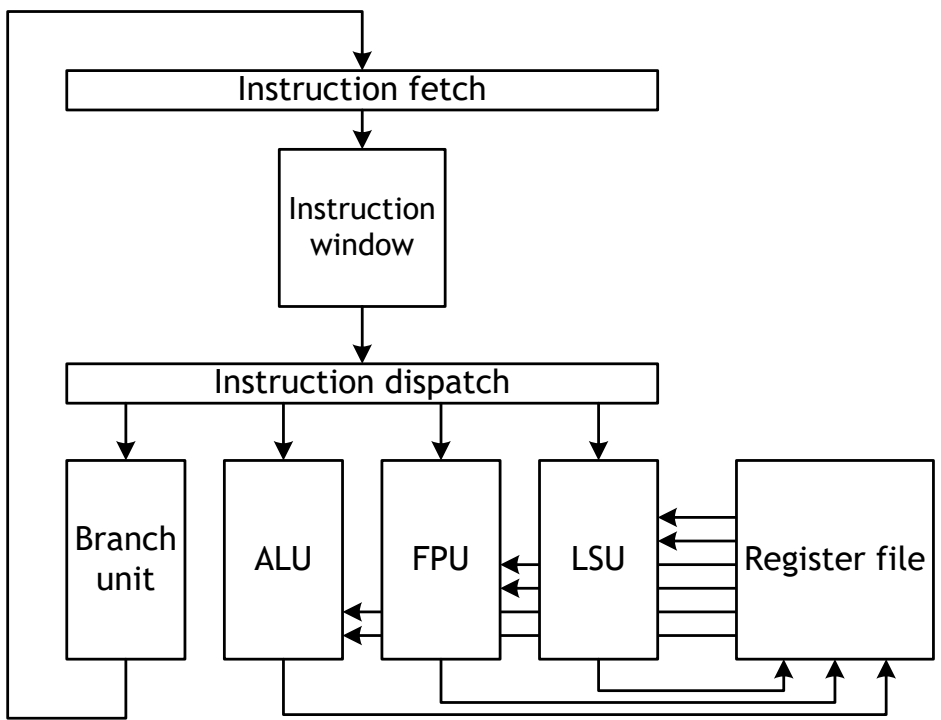

Figure 2.2: Superscalar processor organization.

It's an efficient way of improving performance in general-purpose processors and binary compatibility between processor generations is easily maintained, since the instruction scheduling is performed dynamically by the hardware. The possible performance improvement is primarily limited by the available parallelism in the instruction stream and the complexity of the dependency checking logic. In practical implementations one can expect diminishing returns after 6-8 parallel execution units and the complexity of the hardware increases rapidly, which is one of the main drawbacks. The dependency checking hardware consumes area and energy, which is why it is often avoided in low-power designs.

In a VLIW architecture, shown in Figure 2.3, instructions are grouped in a very long instruction word and executed in parallel on the hardware. The instruction scheduling is done by the compiler instead of the hardware, and the compiler guarantees that sub-instructions in the long instruction word are independent. The VLIW architecture is popular in 


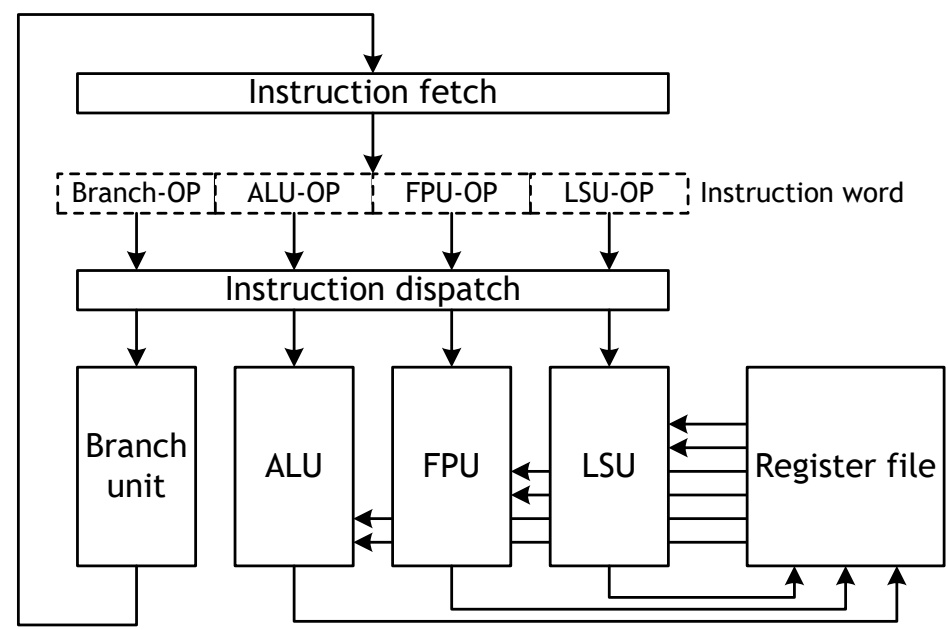

Figure 2.3: VLIW processor organization.

many low-power designs, since the dependency checking logic required in superscalar processors is not necessary. Binary compatibility is however hard to maintain over processor generations, if the number of execution units is modified. Also, since the instruction schedule is static, it is harder to do any useful work when a stall is encountered. This may be especially troublesome when a load-instruction results in a cache-miss.

Superscalar and VLIW processor designs can quite significantly improve performance over simpler designs. However, they have some drawbacks in common. To expose enough instruction-level parallelism, the compiler must often unroll loops and use software pipelining techniques, to efficiently use the hardware. This results in increased code size. The pressure on the register file is high, since all execution units may have to access it simultaneously. Also, it turns out that compilers typically are not able to fully utilize the potential of these architectures, which is why many platforms are shipped with hand-optimized kernel libraries. 


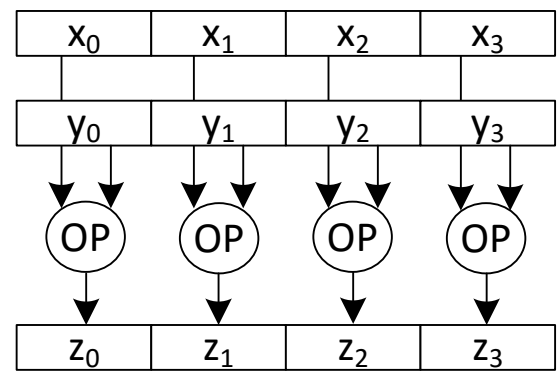

Figure 2.4: SIMD execution.

\subsubsection{SIMD and vector architectures}

Single instruction multiple data (SIMD) instructions perform the same operation on multiple data elements in parallel, as shown in Figure 2.4. A very similar concept is implemented in vector machines. The main difference is that SIMD instructions typically operate on fixed-length vector registers, whereas vector machines supply the number of elements as an instruction input parameter. Both techniques implement DLP inside a processor core and the potential speed-up is equal to the number of parallel execution units. The cost of instruction fetch and control is amortized over multiple operations, which improves energy efficiency. Vector architectures are nowadays less common, but SIMD instructions are commonly available in modern architectures [21, 22].

Early vector architectures often operated directly on off-chip main memory. The increasing performance gap between processor cores and off-chip memory has made this less attractive and most modern architectures instead operate on vector registers. To use a SIMD instruction, one must first load input data to vector registers, possibly align or shuffle operands to prepare for SIMD execution and then perform the SIMD operation. Additional shuffling of the results could also be necessary. Because of this overhead work, the practical performance improvement may be a lot less than expected.

SIMD instructions may be very effective to improve performance and 
energy efficiency. Their effectiveness are mainly limited by [23]:

- The application has limited data-level parallelism.

- The code is dominated by supporting instructions, such as data access and control.

- Unaligned- and out-of-order memory accesses are slow.

- Gathering data into SIMD registers is slow.

- The number of elements to process is not a multiple of SIMD processing width.

- SIMD instructions are not automatically emitted by the compiler.

Despite these drawbacks, 8-way SIMD architectures can typically improve performance for appropriate workloads with 2-4 times, if SIMD instructions are used explicitly. Unfortunately, automatic emission of SIMD instructions by compilers may be less successful [24].

\subsubsection{Multi-core architectures}

Multi-core architectures exploit TLP by executing multiple threads simultaneously on different processor cores. By doubling the number of cores one effectively doubles the theoretical performance of the system. Alternatively one may increase the number of cores and correspondingly decrease the clock frequency, to improve energy efficiency. To fully utilize a multi-core architecture, one must be able to divide the application into a suitable number of (mostly) independent tasks/threads. Multicore architectures are primarily limited by their shared resources, typically memory, and how often the threads they execute must communicate. Multi-core architectures are nowadays very common and have become essential for the success of many systems [25]. 


\subsection{Heterogeneity}

Slow improvements in single-core performance has been a reality for a long time and some algorithms only benefit slightly from homogeneous multi-core parallelism. One popular trend is therefore to use heterogeneous architectures [3]. Performance and energy efficiency can both be improved by specializing different cores for different kinds of tasks. Heterogeneity can show up in several forms, and this section will describe some of the most common ones.

\subsubsection{Single-ISA multi-core heterogeneity}

One type of heterogeneous multi-core architecture is single instruction set heterogeneous multi-cores. All cores use the same instruction set, but the cores may be organized differently to prioritize performance or energy efficiency. The main purpose of these designs is not to improve peak performance of the system, but rather to improve energy efficiency by using more energy efficient cores when performance requirements are not as strict [26]. This approach has become popular for application processors in mobile phones.

\subsubsection{Multi-ISA multi-core heterogeneity}

In contrast to the single-ISA architectures, multi-ISA architectures use different instruction sets for different cores. This implies some sort of instruction set specialization towards the application or the typical workload. These architectures may improve energy efficiency through their specialization, but may lose in generality. Software programming is affected by another level of difficulty, which is to select the most appropriate core for any given task. Sometimes the cores are specialized to such an extent that the best mapping is obvious, but in general it may be very difficult. 


\subsubsection{Execution unit specialization}

One approach to heterogeneity is to start from some architecture and specialize it towards some given source code. One may analyze the source code for hot-spots and devise specialized hardware to execute those hotspots. An example of such an architecture is the GreenDroid architecture [27]. At any given time, the processor executes its instruction stream either using the standard datapath, or, when a hot-spot is encountered, at a hot-spot-specific accelerator. The standard datapath and specific accelerators are all directly connected to the local data cache for fast switching. Energy efficiency may be improved by an order of magnitude.

\subsubsection{Full-custom platform heterogeneity}

For a given application with many different known workloads one may devise a highly customized multi-core architecture with many heterogeneous cores and accelerators. A typical example is a mobile phone system on a chip (SoC). In such an architecture, each accelerator or processor core may be highly specialized and aggressive power management is often used. The energy efficiency may be several orders of magnitude higher than a homogeneous general-purpose chip.

\subsection{Memory hierarchy}

The memory hierarchy of a processor architecture has a huge impact on its performance and energy efficiency, as well as its programming. This section will briefly discuss the most important characteristics of memory hierarchies.

\subsubsection{Shared memory and distributed memory}

One of the most important design choices is whether an architecture should use a shared memory or a distributed memory hierarchy. In a shared memory subsystem, shown in Figure 2.5(a), all processors process data in the shared main memory. In a distributed memory design, 


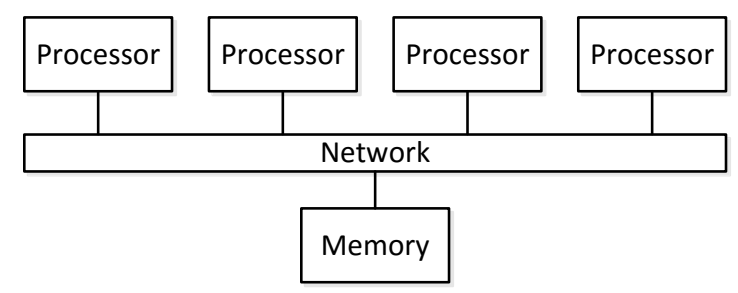

(a) Shared memory system.

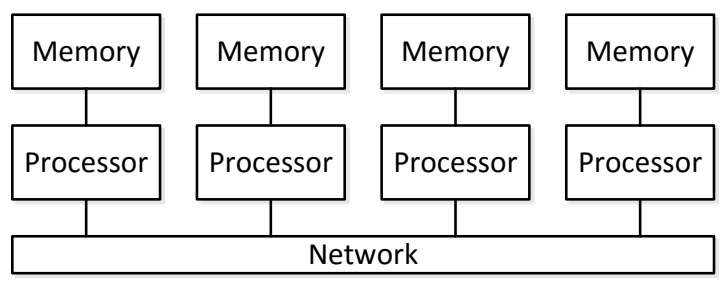

(b) Distributed memory system.

Figure 2.5: The organization of a shared memory system and a distributed memory system respectively.

shown in Figure 2.5(b), each processor has its own private memory and it can only operate directly on this memory. Communication with other processors is explicit.

In practice, many modern systems use a shared memory model, but with physically separated local memories. All processors share a common address space, but keep copies of recently used data and instructions in private caches or cache hierarchies. The main reasons are that the main memory load is significantly decreased, but also the fact that small on-chip caches may be significantly faster compared to off-chip main memory. It is equally common that distributed memory architectures, in addition to their own private on-chip memory, have a single off-chip main memory space for large scale storage.

Table 2.1 compares shared- and distributed memory. In a shared memory system, the main memory will be a potential bottleneck. If caches are introduced we may improve performance, but execution time 
Table 2.1: General characteristics of architectures based on shared memory and distributed memory respectively.

\begin{tabular}{l|cc}
\hline \hline Property & Shared memory & Distributed memory \\
\hline Performance & Low & High \\
Predictability & Low & High \\
Hardware cost & High & Low \\
Energy consumption & High & Low \\
Scalability & Low & High \\
Programming & Easy? & Hard? \\
\hline \hline
\end{tabular}

could become unpredictable, which complicates the implementation of signal processing applications with real-time constraints. In addition, even though we improve performance, data is fetched on demand when a cache miss occurs, which can stall the processor for hundreds of clock cycles in the worst case. In a distributed memory architecture, data is fetched using explicit communication, which may be done before the data is needed to minimize waiting time.

The hardware cost of shared memory is typically higher, because of the hardware overheads associated with cache memories, but also since copies of the same data may exist in multiple levels of the cache hierarchy. The energy consumption will also be higher. The main reason for using shared memory is programming simplicity, but whether or not it is true depends on the circumstances. It is possibly simpler to generate a basic implementation of some algorithm on a shared memory system. However, if one wants fully utilize the architecture, one must carefully optimize the code, which in general is not easy at all. On the other hand, distributed memory architectures may not be suitable for applications where communication is unpredictable or when the algorithms are not easily broken down into smaller pieces that can be transferred efficiently using explicit communication. 


\subsubsection{Memory coherency and memory consistency}

Two related and easily confused properties of memory are memory coherency and memory consistency [28]. Coherency is a property of an individual memory location, that is whether a write to that memory location will eventually be seen by all other processors or not. Consistency on the other hand refers the order of all memory accesses to any memory location. A strict memory consistency model behaves more predictably, but performance may be lower, whereas a weaker memory consistency makes programming more difficult, but performance may be better.

Memory coherency and memory consistency are mostly discussed in shared memory systems. In these systems there is typically hardware support to implement cache coherency and some form of memory consistency model. This ensures that multiple processors can do co-operative execution and communicate through this shared resource. In a distributed memory system, the memory is instead under the programmer's control. Consequently we do not require extra hardware for coherency and consistency.

\subsubsection{Memory bandwidth}

The memory bandwidth of a computer systems is very important for its performance and many applications and algorithms are bound by memory performance rather than compute performance. It's also very easy to generate code that becomes memory bound unnecessarily. In case the system has a hierarchy of memories, such as a cache hierarchy, the memory bottleneck can occur at any link in the hierarchy and one must carefully trade-off the available bandwidth at every memory access link.

There are many techniques one could use to increase memory throughput, such as using wider busses, increase bus frequency, divide memories into multiple memory banks or use multi-port memory. Most techniques however come with a quite significant cost, especially if memory is located off-chip. As discussed earlier, data movement is also very energy consuming and should be avoided if possible. These are two good rea- 
sons why many systems have quite restricted memory bandwidth.

Since there is a limit to how much speed-up one can practically expect from multi-core parallelization, and the fact that clock frequency is not increasing, processor designers are moving towards heterogeneous designs and application-specific instruction set additions. When the instruction set is extended with more powerful instructions, the load on the memory subsystem increases, since less cycles is spent on the actual computing. The logical conclusion is that memory bandwidth must increase in order to avoid memory bottlenecks. The biggest issue is off-chip main memory, which easily can become a bottleneck for the entire system. Since it is hard to circumvent this issue and expensive to simply increase the bandwidth, it is necessary to find ways to utilize local memory bandwidth as much as possible and avoid using the off-chip memory. Algorithms that take this limitation into account are likely to be much better choices in future computer systems, as we can expect that this issue will increase.

\subsection{Energy reduction techniques}

This section will discuss several techniques to reduce energy consumption. We will start by quantifying the energy consumers in a processor.

There are several ways to breakdown processor energy consumption. One way is based on components, such as memories, datapaths and register files. This breakdown makes it hard to separate necessary work from overhead work. In this context we consider datapath computing to be the actual work done by the processor. Data delivery is necessary, since the datapath needs data to work with, but from a performance perspective it is best if this can be performed in parallel with datapath computing. Instruction delivery on the other hand, does not do any useful work and we consider it overhead. It simply tells the processor what to do. Instruction delivery energy can be reduced through better instruction set design or by reducing programmability, until we end up with a completely non-programmable ASIC. 


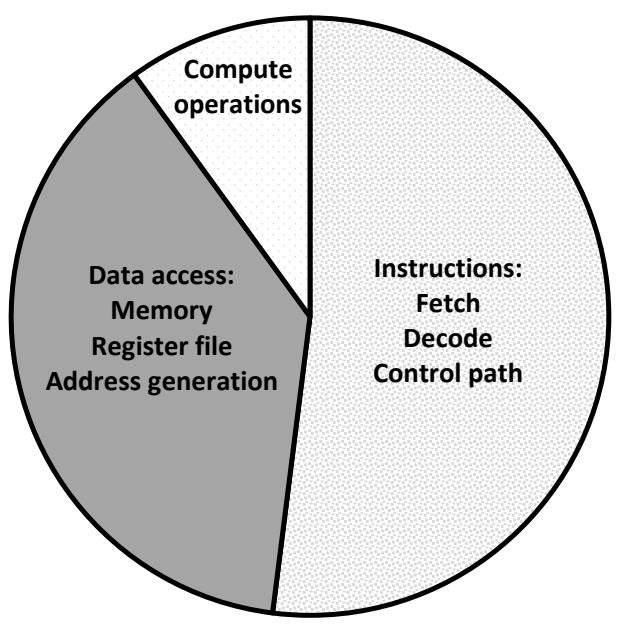

Figure 2.6: Power consumption breakdown of a simple single-issue RISC-DSP.

Based on a simple single-issue RISC-DSP design developed by our research group, power consumption can be roughly broken down as in Figure 2.6. Similar values have also been reported by others [29, 30]. If the processor can execute several instructions per clock cycle (e.g. a superscalar or VLIW architecture), the data consumption pace and computing pace increase accordingly and the energy consumption ratios may stay roughly the same. It is clear that instruction fetching is power hungry. It includes reading instructions from memory, but also instruction decoding and the dynamic power consumed by switching in the processor's control path. It is obvious that if an instruction can instruct the processor to do more work, we can spend more energy on the actual work that we want to perform. Adding more complex instructions and applicationspecific instructions seem to be good ways to achieve this effect.

\subsubsection{Voltage- and frequency scaling}

As discussed in Section 2.1, the parameter which has the largest impact on energy consumption is probably the supply voltage and it affects both dynamic and static energy consumption. The main issue is that a reduced supply voltage will also require clock frequency reduction, to en- 
sure that the circuit operates correctly. A reduced clock frequency results in lower power consumption, but also reduced performance.

Today it is very common that systems use dynamic voltage and frequency scaling (DVFS), to modify the supply voltage and clock frequency at run time [31]. The idea is to avoid running the circuit faster than it needs to. For a signal processing system, especially one with real-time constraints, the goal is to run the circuit fast enough to meet real-time deadlines, but not faster than this, since we will waste energy. This technique is not the main focus of this work, but it is essential for a commercial system.

\subsubsection{Clock- and power-gating}

The clock network is a large energy consumer, primarily because it is constantly switching and must be distributed to all flip-flops in the design. In some designs it may be the source of almost half of the chip's energy consumption [32]. Clock gating is a very useful technique to reduce the energy consumption. It may be applied in a course-grained manner, e.g. to turn off the clock to an entire inactive processor core, or at a fine-grained level, at the level of individual flip-flops.

Another useful technique is power gating, that is to turn off the power supply for inactive circuits. Power gating is typically under the control of some power management unit (PMU) which tries to reduce static power consumption. Like clock gating, power gating can also be applied at a course-grained and a fine-grained level. One interesting technique is to turn of the power to unused execution units [33].

This thesis will primarily focus on reducing energy consumption of active circuits, thus we will not discuss course-grained clock- and power gating at any length. Fine-grained clock gating may be applied automatically by synthesis tools. Although fine-grained power gating is useful for our purposes as well, this is outside the scope of this thesis and it will only be discussed briefly. 


\subsection{Summary}

This chapter reviewed parallel processor architectures and their memory hierarchies, and discussed processor design from an energy perspective. Key insights include the fact the static power consumption no longer can be ignored and that instruction and data movement is much more power hungry than actual computing. This implies that micro-optimizations for reduced power consumption in processor datapaths may only give marginal benefits, if data delivery, and especially instruction delivery, are not attacked first. 


\section{Chapter 3 \\ DSP application characteristics}

In order to design an appropriate DSP processor for a given application, it is important to know the application's computing characteristics. A key element is to identify the algorithms to be executed, but other properties such as the required data types and memory requirements must also be determined. Since one of the main goals of this research is to design a base system suitable for many applications, we are very much interested in what different DSP applications have in common. This will be of great value in the design process.

\subsection{Digital signal processing systems}

The idea of digital signal processing is to digitally manipulate some physical signal [4]. A basic digital signal processing system is shown in Figure 3.1. The physical signal, e.g. air vibration or electro-magnetic radiation, is measured by some sensor, which turns the physical signal into an analog electrical signal. The analog signal is then digitized by an analog-todigital converter (ADC), which samples and quantizes the signal at regular time intervals. The digital signal may then be processed by a digital signal processor, after which it can be converted back to a physical signal, 


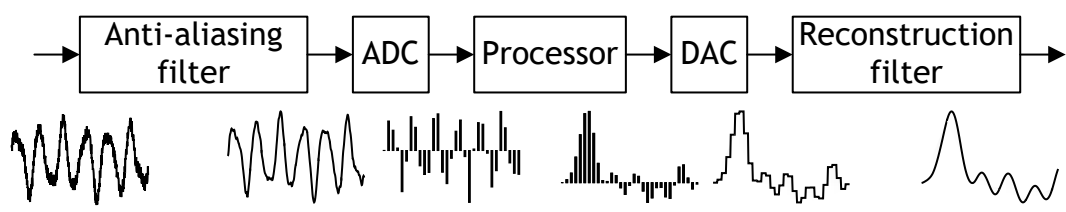

Figure 3.1: A digital signal processing system.

through a digital-to-analog converter (DAC) and an actuator or visual representation system. In addition, it is also common that analog filters are inserted before the ADC, for anti-aliasing, and after the DAC, for reconstruction. Other systems are also possible, such as systems designed to simply process some input for long-term digital storage. However, this system setup represents the vast majority of actual systems.

\subsection{Application examples}

This section will present some common signal processing applications. While this is far from an exhaustive review, it will give important insights in application requirements and shared features.

\subsubsection{Software-defined radio}

A software-defined radio (SDR) system implements most of the radio signal processing in software, as opposed to fixed hardware solutions. It allows higher flexibility than fixed circuitry and hardware multiplexing in multi-standard scenarios. The performance and energy efficiency requirements are very high, and many systems run on battery power [34].

Most of the computations are done on relatively long vectors of complex data, and the control flow is often simple and predictable [9]. Many algorithms may be implemented with fixed-point operations, although some operations in newer communication standards are more easily implemented with floating-point operations, such as channel matrix inversion. There are strict real-time requirements to guarantee correct operation [35]. In addition, software-defined radio systems are often fitted 
with several accelerators for tasks that do not map efficiently to processors, e.g. Turbo decoding [36]. Important algorithms include many types of filters and Fourier transforms (FFT/IFFT).

\subsubsection{Media processing}

Media processing primarily involves processing of audio and video in real-time. A typical example is decompression of compressed video formats to be displayed on a TV screen, computer or mobile device. Operations are often performed on smaller blocks of pixels, typically $4 \times 4,8 \times 8$ or $16 \times 16$ squares and there is plenty of data-level parallelism [37]. The working set is typically one or multiple video frames, which requires off-chip storage.

Media applications may require flexible addressing for best performance and a lack of support for unaligned memory accesses can result in unacceptable levels of performance degradation [38]. Filtering and transformations are common, as well as bit-level operations, typically mapped to accelerators in more efficient system designs [39].

\subsubsection{Image processing}

Digital image processing encompass a wide variety of applications with different computing characteristics, including simple filtering, transformations, color space conversion or image compression, but also more complex operations such as classification, pattern recognition and feature extraction [40, 41]. It may be used for any number of purposes such as automatic reading of car number plates or road signs, real-time inspection in product manufacturing or inspection of medical images or satellite images. Many operations are massively parallel and are excellently suited for wide-SIMD and/or multi-core architectures. Data types vary, all the way from bit-level processing to floating-point. 


\subsubsection{Radar}

Modern radar systems use digital signal processing to implement features such as object identification, tracking and targeting. The dynamic range of the signals can be high and floating-point is useful. Filtering and transforms are common and there is plently of data-level parallelism.

\subsection{Common characteristics}

This section will summarize the most important common features of DSP applications.

\subsubsection{Real-time constraints}

Communication systems typically have timing constraints that must be followed and media applications require that the audio/video signals can be processed fast enough to produce an acceptable frame rate. Failure to comply with the timing constraints results in loss of quality, such as noticeable stuttering in video playback or a communication system that is unable to transmit or receive any information. Real-time constraints are very commonly found in DSP applications.

\subsubsection{Vector/matrix operations}

Most signal processing applications have an abundance of data-level parallelism. Operations are performed on vectors or matrices, and it is also quite common that several vectors/matrices can be processed in parallel. One thing that is very important to consider is data access, since several algorithms require out-of-order access to data elements.

\subsubsection{Data types}

To reduce hardware cost, improve energy efficiency and increase performance, most signal processing implementations try to use data types which require as few bits as possible [4]. Hardware support for 64-bit 
data types and floating-point is often omitted. This may increase the software design effort, but the benefits can make it worthwhile.

The current trend is that DSP processors are designed with hardware support for larger word lengths and floating-point is increasingly common. One explanation is that the energy cost of floating-point operations is not as high anymore. Two other reasons are that newer applications standards may have higher dynamic range requirements and the use of floating-point can sometimes reduce the time-to-market. Fixedpoint DSPs are however still widely available on the market.

\subsubsection{Data access patterns}

Many data access patterns show up frequently, such as 1D vector access, $2 \mathrm{D}$ and $3 \mathrm{D}$ block accesses, row-major and column-major vector access of matrices and strided memory accesses. Some specific addressing patterns, such as bit-reversed addressing patterns are of great importance. However, the size of the vectors and matrices vary significantly, as well as the memory strides, which makes flexible data access capabilities a requirement if a wide range of applications should be supported.

\subsubsection{Computing kernels}

Most DSP applications are very much characterized by the fact that the overall execution time is dominated by the execution of a smaller set of kernel routines [42]. Typical kernels are shown in Table 3.1. These routines may be used so frequently that their efficient implementation is crucial to the success of the hardware platform. This property can also make it simple to estimate the performance of the application, if the performance of the individual kernels is known.

Multiply- and accumulate (MAC) operations are very central. Many common filters, transforms and vector/matrix operations can be implemented with essentially only MAC operations. There are excellent opportunities for adding application-specific instructions to improve performance even more. 
Table 3.1: Typical digital signal processing kernels.

\begin{tabular}{l|l}
\hline \hline Category & Kernel \\
\hline Simple element-wise & add/sub/mul/min/max/and/or, ... \\
Reductions & sum, min/max, dot product, ... \\
Filters & FIR, IIR, interpolation, decimation, ... \\
Transforms & FFT, DCT, Walsh, Hadamard, ... \\
Math functions & $1 / x, 1 / \sqrt{x}$, sin, cos, exp, log , ... \\
Vector operations & sort, interleave, prefix, ... \\
Matrix operations & mul, transpose, inversion, \\
& QRD, LUD, SVD, ... \\
Table access & table lookup, histogram, ... \\
\hline \hline
\end{tabular}

\subsubsection{Predictability}

Many DSP computing kernels are highly predictable in several ways. The implementations of e.g. filters and Fast Fourier Transform, have predictable control flow and statically known compute- and data access patterns. Predictable in this sense does not necessarily mean that the execution time of the kernel will be predictable. However, if the data access patterns are predictable and the hardware design doesn't introduce unpredictability (such as possible cache misses), the execution time of the kernel can be determined very precisely (or exactly). This is very useful to guarantee that real-time constraints can be met. There are of course exceptions, such as iterative methods in digital image processing.

In addition to predictable kernels, many applications are also predictable on the application-level. The order in which kernels should be invoked or the time when different data blocks is needed may be known statically. These properties makes it possible to design hardware architectures that allow static processing pipelines or explicit data movement, so that data is available where it needs to be before actual use. 


\subsection{Summary}

This chapter reviewed the characteristics of some typical signal processing applications. Most applications are characterized by the execution of a smaller set of important kernels that have abundant data-level parallelism. The same kernels show up frequently. The kernels, but sometimes also entire applications, are typically very predictable in terms of control flow and data access patterns. This property is very useful when designing an efficient DSP architecture. 


\section{Chapter 4 Research methodology}

This chapter will summarize the research methodology used in this research project. To some extent it will cover the creative process and discuss how the research methodology changed as the project progressed.

The ePUMA research project as a whole is divided into four separate projects:

- System-level hardware: Multi-core architecture and network-onchip.

- Core-level hardware: Processor core design.

- System-level tool-chain: Multi-core programming tools.

- Core-level tool-chain: Tools and programming languages for DSP kernel design.

Since this thesis primarily involves the core-level hardware and corelevel tool-chain, this summary will focus on those parts. The whole research project and its components are summarized in Appendix A.

\subsection{General methodology}

The overall design process is very much influenced by the analysis of DSP applications, but also the fact that it should be possible to special- 
ize the architecture for a specific application. The design process can be summarized as:

1. Analysis of DSP applications and related work. Identify areas for improvement.

2. State the goals and scope for the architecture.

3. Design the configurable base architecture and decide the configurable parameters. Create a proof-of-concept demonstrator.

4. Choose an application. Identify suitable kernels.

5. Map application and kernels to the platform. Modify architecture and tool-chain if necessary. Evaluate!

6. Retain successful kernels, instruction set additions and other modifications in the platform design database for future use.

7. Repeat from step 4, until enough applications are covered.

8. Evaluate platform by configuring the architecture for different applications.

In reality, the development process has not followed this process strictly. In practice, several applications have often been investigated in parallel, often by different people. Steps 4-7 can easily turn into an endless loop and practically one must decide when to stop. Fortunately, the fact that DSP applications share many similarities saves a lot of work. Also, before the actual hardware RTL code is written, the application and kernels can be evaluated in architectural simulation.

\subsection{Kernel-based hardware/software co-design}

The methodology used for processor core-level design is centered on efficient implementation of DSP compute kernels. During the course of the research project, two different approaches have been used. The first one can be summarized as: 
1. Select a new kernel for implementation.

2. Check if the kernel can be efficiently executed with the already available hardware, if so goto 4 .

3. Modify address generators and/or implement new processor instructions.

4. Write the kernel code and benchmark the implementation. If not $\mathrm{OK}$, try alternative implementations.

\section{Done!}

Although good performance can be achieved with this method, the focus is on the hardware and it disregards the software implementation. In practice many kernels may have to be implemented in assembly. In contrast, the following methodology puts equal emphasis on hardware and software:

1. Select a new kernel for implementation.

2. Create a kernel implementation in a "pseudo"-high-level language, an implementation which represents the desired final kernel code.

3. Decision: Can the kernel be compiled, or should it be supported (partly/fully) by an optimized library implementation?

4. Modify arch. and/or compiler if necessary. If the kernel should be implemented as optimized assembly, do so.

5. Benchmark kernel. If not OK, go back to step 2.

6. Done!

This approach starts from a software prototype and envisions a kernel implementation that can be compiled. As the research progressed, this approach turned out to be much more successful. 


\subsection{Retrospective}

Many things can be said about the research methodology. A too strong focus on hardware can turn out to be extremely limiting. Early on it may seem that the programming is manageable, but when application complexity increases, the programming productivity can turn out to be very low. A hardware/software co-design approach is likely to be much more successful, as it turned out in our case. When using a "pseudo"-high-level language implementation or kernel model to guide the development, it is easier to envision the final implementation of both hardware and software. It gives a clearer sense of direction and especially one ensures that a high-level language version is feasible. It is very important to consider the programming model and tool-chain as early as possible. 


\section{Part II}

\section{The ePUMA architecture}





\section{Chapter 5}

\section{The ePUMA architecture}

This chapter will describe our demonstration platform ePUMA, and the thought-process behind its implementation. The focus will be on the system-level hardware and system-level tools, while the core-level hardware and tools will be left for later chapters. Although the research presented in this thesis primarily discusses core-level issues, it is still very important to understand the system-level architecture, since it strongly influences the core-level design. The full list of the ePUMA hardware/software components is given in Appendix A. The ePUMA platform has undergone several redesigns over the years, all of which are summarized in Appendix A. This chapter will focus on the latest design.

\subsection{Design space exploration}

Since one cannot possibly implement and evaluate the consequences of every design decision in a large design such as a processor platform, some of the decisions have to be based purely on intuition. That is also the main purpose of this section, to discuss different design choices and disregard some possibilities, simply to limit the design space. Chapters 2 and 3 will serve as the basis for the decisions and the architecture goals will be presented. 


\subsubsection{Compute/control separation}

As discussed in Chapter 3, DSP applications are often dominated by kernel execution. The kernels are characterized by predictable computing on vectors and matrices and statically known data access patterns. The control structures are often simple and require only simple iteration. The application that invokes a kernel may be arbitrarily complex, but once a kernel is running it can run independently. This is an excellent opportunity for parallel execution. Based on this observation, it is clear that execution can be divided into compute work and control work, and these tasks could be run separately on compute cores and control cores respectively. This makes it possible to specialize the cores for their specific purpose. Some generalizing characteristics of a compute core could be:

- SIMD/vector-style execution: The cost of instruction delivery is amortized over multiple operations and performance is improved.

- Complex datapath: An application-specific datapath with complex instructions ensures that a large number of arithmetic operations is carried out every clock cycle and energy efficiency is improved [30].

- Memory parallelism: Multiple memories, multi-bank memory or multi-port memory may be utilized to avoid memory bottlenecks. A vector datapath may require multiple vector memory accesses every cycle to achieve full utilization.

- Long pipeline: Due to the simple control structures of compute kernels, a long pipeline may be used to utilize pipeline parallelism.

A control core may instead benefit from the following properties:

- Simpler RISC pipeline: A control core may typically execute compiled C code. However, as long as control work is not dominating the application, such a processor can be made small and simple to reduce hardware cost and to improve energy efficiency. 
- Single memory space: By using a single memory for data and instructions, it is possible to compile standard reference $C$ code without making any modifications.

- Shorter pipeline: The code may be more unpredictable than compute kernel code. Since it may execute complex control flow, a shorter pipeline with lower penality for unpredictable jump instructions may be useful.

If the application is split into control work and compute work, we are more likely able to fully utilize expensive resources such as vector memories and vector datapaths. A smaller processor core will be more energy efficient for smaller compute and control tasks, whereas a maximally utilized compute-optimized vector core can perform much work very quickly. Figures 5.1(a) and 5.1(b) compares the execution on homogeneously and heterogeneously configured multi-processors. The homogeneous processor time-interleaves control work and compute work, whereas the heterogeneous uses the control cores and compute cores for their specific purposes. The wide vector datapaths are highly utilized on the compute cores and area/energy can be saved with the smaller control processors. If the application is very compute-heavy, one may couple multiple compute-processors with each control processor, as shown in Figure 5.1(b).

It is much less obvious how control- and compute cores should be physically connected and how they should interact. A tight integration has some obvious benefits, such as a lower cycle time cost for starting and stopping compute jobs on a compute core. This means less overhead, especially in case the compute jobs are short. The integration will be further discussed in Section 5.2.1.

\subsubsection{Memory subsystem}

Based on our focus on energy efficiency and performance, a distributed scratchpad-based memory subsystem may be the best choice for an efficient DSP system. It is clear that exposing the memory hierarchy to the 


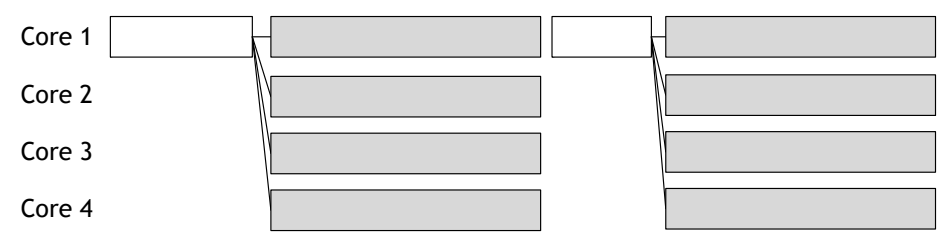

(a) Homogeneous multi-processor system.

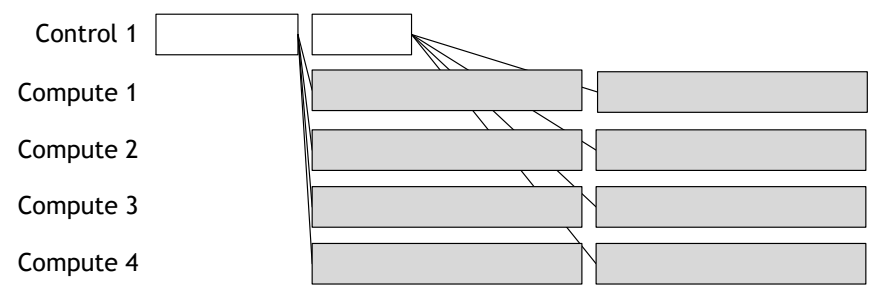

(b) Heterogeneous off-loading multi-processor system.

Figure 5.1: Comparison of homogeneous and heterogeneous processing.

programmer and tool-chain can have some significant performance benefits. It also exposes data locality directly, which can be used to improve energy efficiency.

There are many reasons why a shared memory cache-based architecture is less appropriate [43]. First of all, scratchpad memories are cheaper in terms of area, since they don't require tag arrays or coherency hardware. In addition, if the cache hierarchy has multiple levels and a particular data element is cached in the innermost cache-level, a copy of the data also exists in the outer level caches. The result is that the available on-chip memory can never be optimally utilized. In addition, caches consume more energy. Reading the tag array may consume 33\% additional energy in relation to the cache's data area and the cache controller can be even more energy hungry [44].

A scratchpad-based memory system has several other advantages, in addition to the area and energy efficiency benefits. It behaves pre- 
dictably, since the programmer is responsible for data movement. Data transfers and compute operations can be scheduled in larger blocks that overlap in time, which is more efficient than using smaller memory transfers with current memory technology [45]. The core-level hardware design can also be simplified. Since local data access is predictable and the data is always available, the core-level hardware does not have to deal with caches misses. The hardware cost and verification time is reduced. The predictability issues with cache-based systems can partly be eliminated by techniques such as cache locking, but this doesn't eliminate the extra hardware cost and energy consumption overhead.

The downside of the scratchpad-based architecture is possibly the programming effort. Also, it may not be the best choice if the application is unpredictable. If a memory access cannot be predicted before the data is needed, preloading of data may not be possible and shared memory may be more suitable than programmer-scheduled data transfers. Still, since our assumption is that most of the interesting applications will be predictable, there is much to gain by using a scratchpad-based memory subsystem.

\subsubsection{Summary of architecture goals}

Based on the previous discussion, some design goals were set early for ePUMA:

- Orthogonalize computing, data access and control - the cycle time cost of data access and control should be hidden behind compute operations.

- Predictable hardware behavior and predictable execution time - reduces hardware cost and simplifies hardware and software design.

- Reduce energy consumption - especially target the energy cost of instruction delivery, but also provide means to reduce unnecessary data movement. 


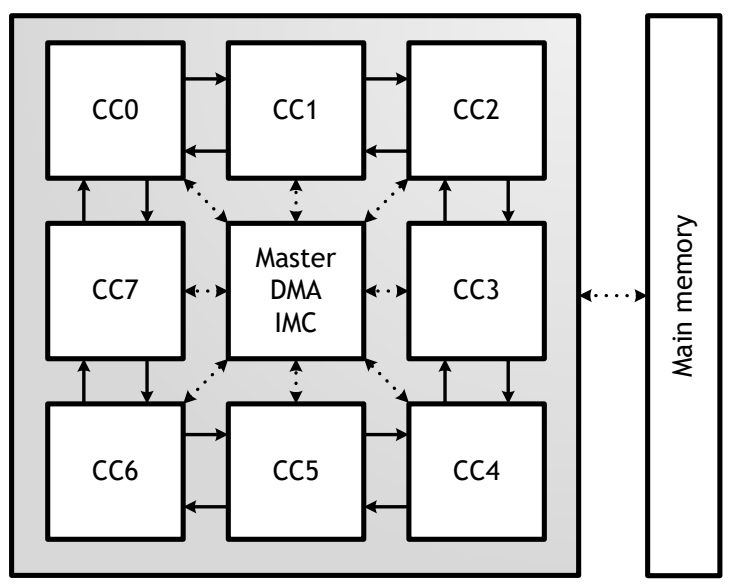

Figure 5.2: ePUMA architecture overview.

- Complex instruction datapath - performs many operations per cycle on vector/matrix data and bridges the semantic gap between hardware and high-level languages.

- Flexible local data access - support flexible addressing capabilities to cover many applications and eliminate the problems with data access in traditional SIMD/vector architectures.

- Assembly-free kernel implementation - provide an alternative kernel programming approach to avoid assembly programming.

Although some goals may not be possible to fulfill entirely, they provide a clear sense of direction.

\subsection{The ePUMA hardware architecture}

A block diagram of the ePUMA platform is shown in Figure 5.2. The main components are the master processor and up to 8 compute clusters, interconnected by a star network, a bidirectional ring-network and a serial bus [7]. The master processor controls the application, manages the off-chip main memory and offloads computing tasks to the compute 
clusters. The offloading design was chosen simply because of the predictability property of typical applications. In this case it is possible to centralize the control over main memory, to reduce hardware cost and be able to more effectively utilize and schedule the use of the main memory.

The architecture uses distributed memory. The master processor may issue compute tasks to compute clusters by explicitly moving program code and data to the clusters. The master processor on the other hand, runs code residing in main memory through the use of data- and instruction caches. The master uses caches simply because of simplified application development, but also because the additional hardware cost associated with caches for a single master has very little effect on total system cost. Additionally, since we are primarily interested in compute-heavy applications, the possible unpredictability introduced by caches on this level should not influence overall execution time by much.

The hardware architecture of the master processor is a simple RISC processor design. It could, with relative ease, be replaced by a more powerful VLIW or superscalar design, but in the context of this project, the architecture of the master is relatively unimportant. The master has instead been designed for low hardware cost and low power consumption and the main goal is to be able to execute compiled C code.

\subsubsection{The compute cluster}

The architecture of an ePUMA compute cluster is shown in Figure 5.3. In ePUMA's offloading computing model, these clusters act as the main computation resources of the platform. Each compute cluster externally act as a single-core processor. However, internally a cluster is divided into multiple cores dedicated to specific tasks.

A compute cluster contains a local scalar RISC controller, the Compute Cluster Controller (CCC) and a configurable number of Matrix Processing Elements (MPE) and optional accelerators. The controller runs the main thread of execution in the cluster, manages communication with other clusters, manages local memories and off-loads computing tasks to the MPEs. The MPEs are DSP processors optimized for vector and matrix 


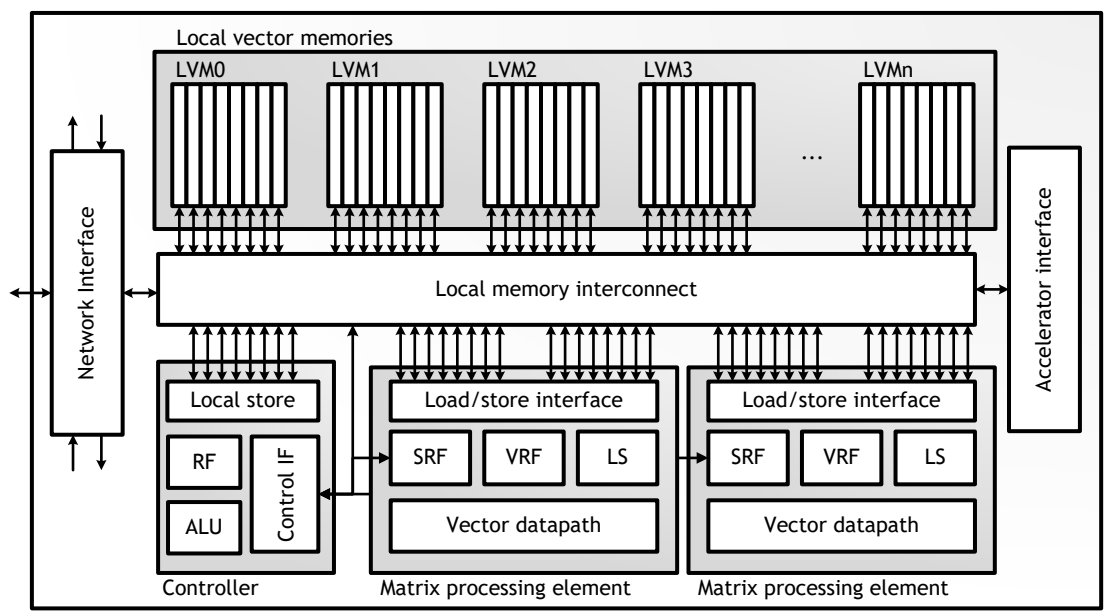

Figure 5.3: Overview of the ePUMA compute cluster architecture.

computing. By executing control- and compute tasks on separate cores, computing and control can be overlapped and the cores can be specialized. An additional benefit is that a system-level programmer can view compute kernels as black-boxes and simply execute them. Since the compute kernels are run on separate compute cores, system programming and kernel development can be done independently.

Local storage of compute data is provided by a set of Local Vector Memories (LVM). The number of LVMs in a cluster can be configured to match the number of compute resources. However, typically one would at least expect 2 or 3 LVMs per MPE. Memory parallelism is essential for maximum performance, since a normal processor instruction with binary operations could require up to three memory accesses ( 2 source operands, 1 destination operand). Each LVM is multi-banked to allow penalty-free unaligned and out-of-order data access, a common problem in many other SIMD/vector processors [46] [47].

The LVMs are connected to the compute resources through a local interconnection network. To minimize data transfer time and data transfer energy consumption, the controller can reassign memory banks to different cores as execution progresses so that no actual data movement has to be performed. One memory bank can also be assigned to the system 
on-chip network which allows overlapped network transfers and computing without interference. In addition to the LVMs, each compute resource stores code and other data in its own local store (LS). This ensures that all resources can fetch instructions and kernel constants without interfering with each other.

Communication between the cluster controller and any compute resource, such as MPEs, is done through shared registers. This, in combination with the reassignable LVMs, ensures that inter-processor communication overhead is minimized. It makes it possible to switch from processor-based processing to accelerator-based processing very quickly, but also from one MPE to another, which makes sense especially if the MPEs are heterogeneously configured.

The MPEs are very central to the ePUMA design. They are the platform's main computation resource and will, in principle, run all DSP computation kernels. Since DSP applications are very kernel-centered, their design very much affect the performance and energy efficiency of the platform. Together with the LVM memories, they will also occupy the majority of the hardware area of a final ePUMA design, typically in the order of $80 \%$. The design of the MPEs is one of the main topics of this thesis and Part III will cover the MPE hardware design extensively. Software related issues, both at the MPE-level and system-level, will be covered in Part IV.

\subsubsection{The memory hierarchy}

The ePUMA memory hierarchy is summarized in Figure 5.4. The off-chip main memory is the main storage resource. The master processor uses a cache-based architecture, whereas the compute clusters uses scratchpad memory storage. As mentioned earlier, each processor in a compute cluster has its own local store, primarily used for program code, and a set of LVMs for compute data. The lowest level in the storage hierarchy are the processor/accelerator registers. 


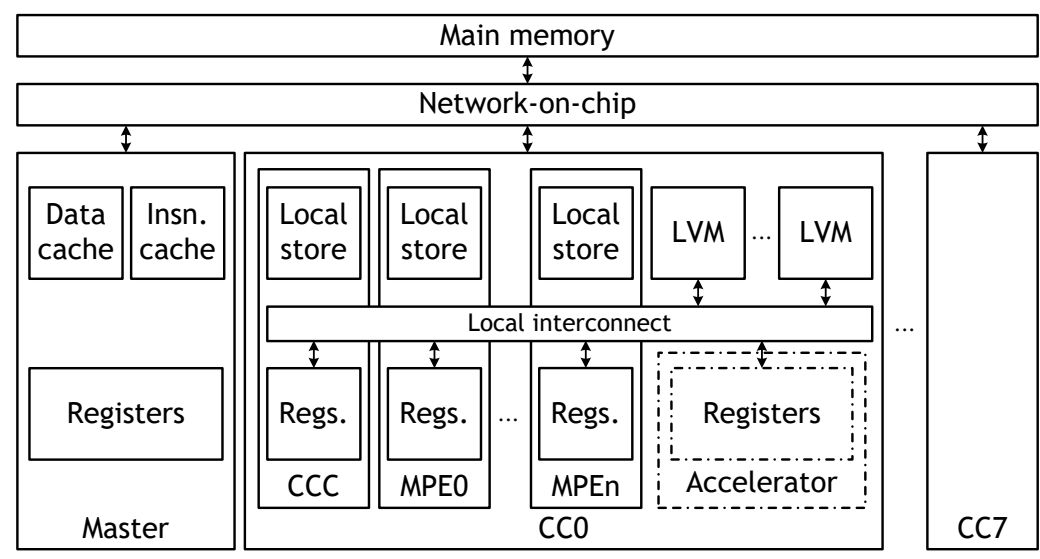

Figure 5.4: ePUMA memory hierarchy.

\subsubsection{The network-on-chip}

The ePUMA NoC connects the main memory, master processor and compute clusters. From a high-level perspective it shares many similarities with other networks, such as the STI Cell's network [48]. It's divided into the following sub-networks:

- Star network: The Star network moves data between external offchip main memory and compute cluster local memories, including both LVMs and local stores. The Star network supports both pointto-point and broadcast communication [49].

- Ring network: The bidirectional ring network can be used for communication between compute clusters and makes it possible to implement a streaming computing model efficiently. It supports both point-to-point streaming of data as well as broadcast operations [50].

- Serial bus: The serial bus is low-cost serial network designed for low latency mailbox communication. It's mainly used for notification and synchronization between compute clusters. By using the serial bus for shorter messages, one can avoid interrupting long latency stream data transfers on the Ring network. 
The Star- and Ring-networks use a Tx+Rx communication model. This implies a co-operation of transmitter and receiver on the network. When the transmitter/receiver both have issued a send/receive operation, stream data will be transferred and the network can be viewed as a first in first out (FIFO) buffer. The network hardware makes sure that the data communication is synchronized. The network physical links do not carry address information, to save implementation cost. Address generation is instead distributed, where external memory addresses are generated by a DMA controller and local addresses for LVMs and local stores are generated locally in the compute clusters.

The Star- and Ring-networks physical links are 128 bits wide. This was chosen as a reasonable trade-off between performance and cost. 128bit vector computing is also a reasonable trade-off that works well for both SDR as well as image- and video-processing. This value may be configurable in the future.

\subsection{System configurability}

One of the main goals of the platform is configurability. In theory it is a good idea to keep as many parameters as possible configurable, but it also complicates the architecture selection process. Every configurable parameter also increases the implementation effort of the configurable platform design.

Table 5.1 lists the most interesting configurable parameters of the ePUMA platform and some typical values. The most interesting parameters are the number of compute clusters and their individual configurations. In addition one may tune the on-chip-network and change the cache sizes for the system master processor.

Table 5.2 lists the configurable parameters of a compute cluster, where the number of MPEs and the number of LVMs are probably the most interesting design decisions. Also important is the local interconnect network between processor cores and memories. Especially one may want to constrain the possible interconnections to some extent, since a full 
Table 5.1: Configurable parameters of the ePUMA platform.

\begin{tabular}{l|l}
\hline \hline Property & Typical value \\
\hline Number of compute clusters & $2-8$ \\
Network topology & E.g. Ring or Full crossbar \\
Network link size & $128-b i t s$ \\
Master I-cache size & $16-1024 \mathrm{kB}$ \\
Master D-cache size & $16-1024 \mathrm{kB}$ \\
\hline \hline
\end{tabular}

Table 5.2: Configurable parameters of the ePUMA compute cluster.

\begin{tabular}{l|l}
\hline \hline Property & Typical value \\
\hline Number of MPEs & $1-8$ \\
Number of LVMs & $3-24$ \\
LVM size & $1-128 \mathrm{kB}$ \\
CCC local store size & $1-128 \mathrm{kB}$ \\
Interconnect topology & Custom \\
Individual MPE configuration & See Chapter 11 \\
Optional accelerators & - \\
\hline \hline
\end{tabular}

crossbar between all cores and memories may be expensive. All MPEs may be individually configured, which is further described in Chapter 11. The platform's configurability allows a small system to be implemented, possibly with only a single MPE. Maximally, the current design allows 8 compute clusters, each configured with $8 \mathrm{MPEs}$, for a total of 64 MPEs.

\subsection{Programming}

The programming of ePUMA can be subdivided into two separate tasks: system programming and kernel programming. The system-level programming is the process of developing software for the master processor and compute cluster controllers. The system programming process 
is very similar to the programming of other distributed memory architectures, such as STI Cell [51]. C compilers are available for the master processor and the cluster controllers. The main tasks are to divide computing tasks between the compute clusters, decide how communication should be performed and how to use the local vector memories and offchip main memory. The system programmer may call compute kernels to accelerate computing tasks.

Kernels are provided by two means: kernel programming or the kernel library. Using kernels from an already available library of highly optimized kernels should be the best choice in general, but all possible kernels can't be predicted by the platform designer. Still, most computing platforms today provide libraries that handle many types of applications and common operations [52,53]. In case a kernel is not available, the kernel has to be implemented. In this case we would like to avoid assembly programming as much as possible.

Many DSP platforms are exclusively programmed in C and assembly. Assembly is frequently used for the kernels to achieve best performance and utilization of the processor platform. The main problem is that $C$ compilers are not good enough to exploit the hardware automatically and automatic vectorization is far from successful [24, 54].

A C compiler takes high-level language code and translates it into very basic operations, such as additions, multiplications and memory accesses. An optimizing compiler would then try to match these very simple operations with more complex instructions and auto-vectorize if possible. The $\mathrm{C}$ language allows direct access to memory through pointers, which complicates code analysis and may prevent automatic parallelization. This feature is sometimes very useful, but it is not necessarily the best way to express computation.

For ePUMA the approach is different. The goal is to write code at a higher level of abstraction and avoid lowering the level of abstraction in the compiler as well. As an example, if a programming language allows the programmer to use a function or operator that performs e.g. complex radix-4 butterfly operation and the processor has an equivalent instruc- 


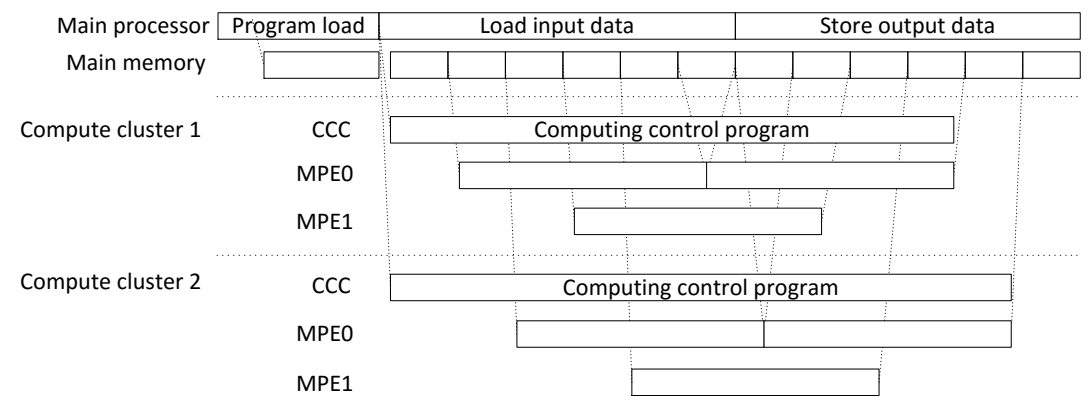

Figure 5.5: Execution time behavior on ePUMA.

tion, this could be automatically emitted. This avoids the cumbersome and possibly not successful step of having to write the butterfly with additions and multiplications and then let the compiler try to match the code with the hardware instruction. As the hardware instruction complexity increases, this makes even more sense.

This introduction only serves as a brief background to ePUMA programming. Part IV will cover this topic in greater detail.

\subsection{Application execution}

This section will describe an execution scenario on the ePUMA system. Figure 5.5 shows a simple Gantt chart of execution time behavior. The system has been configured with two compute clusters, each with two MPEs. A data-level massively parallel application has been chosen for its simplicity, but it represents many practical algorithms, such as image transformations or image filtering. The execution behavior is as follows:

1. Execution starts from the system main processor, which initiates a broadcast of the compute cluster program. In this example, both clusters execute the same program code.

2. The main processor starts the execution on all compute clusters (starts execution on the compute cluster controllers). 
3. The main processor sets up the transfer of the first input data. All clusters wait for input data.

4. When an input data block is received, the cluster controller assigns the input data memory to an MPE and starts MPE computing. Meanwhile, the main processor can load more input data.

5. All MPEs are eventually running. The main processor may continue to send even more input data to spare LVMs. This makes sure that as soon as an MPE finishes a compute job, it may immediately be restarted with new input data.

6. MPEs will eventually have produced output data, which is scheduled for storage to main memory.

7. Finally, the parallel section is finished and the final output data is stored to main memory.

To summarize, the work distribution is as follows: The main processor controls main memory and schedules compute tasks. In this simple scenario it uses a static schedule and the main memory data access pattern is easily predictable. The compute cluster controller manages the LVMs, assigns LVMs to MPEs and starts and synchronizes their execution. The MPEs simply perform computing. The main processor and compute clusters are synchronized by the NoC hardware. Main memory transfers and network communication only commence after both ends of the communication has accepted the communication.

\subsection{Summary}

This chapter described the system-level architecture of the ePUMA platform. The most important features are the clustered compute resources, the compute/control separation, the shared pool of multi-banked local vector memories and the vector compute processors. In addition, system programming and system execution behavior were discussed. Most importantly, this chapter described how the system-level design fulfills the 
architecture goals that were described in Section 5.1.3 and the remainder of this thesis will be devoted to the fulfillment of these goals at the processor core-level. 


\section{Chapter 6 Related work}

This chapter will briefly review other processor architectures and other means of generating an ASIP-DSP. There is certainly no shortage of platforms. For many applications there are specialized designs available, but more general designs are equally common. Some DSP manufacturers produce chips themselves, while others only sell the right to their intellectual property (IP). Since an exhaustive review is far from possible, we look at some of the more interesting platforms, many of them which have similar characteristics as ePUMA.

\subsection{Processor generators}

A processor generator generates a processor platform from some architecture description. There are several alternatives available from both the research community and commercial actors. Some examples are NoGAP [55], LISA [56] and Synopsys ASIP Designer [57].

A processor generator is very much limited by what can be expressed in the architecture description, but if a suitable description can be made, tools such as assemblers, compilers, simulators and RTL code could be automatically generated. Unfortunately, processor generators typically assume that the processor is organized as most other processors, with typical RISC instructions sets and fairly standardized memory hierarchies, to which one can make some application-specific additions. It may 
make it impossible to implement architectures such as ePUMA.

In contrast, if an ePUMA platform instance is generated, many design aspects are already fixed and the design time can be reduced. ePUMA is C programmable on the system-level, but on the kernel-level the focus is to create an architecture for high compute efficiency, rather than good support for $\mathrm{C}$ programming. This allows us to target energy efficiency aspects much more aggressively, such as reducing instruction fetching.

\subsection{Texas Instruments DSPs}

Texas Instruments (TI) offers a wide selection of off-the-shelf DSPs, such as general-purpose fixed-point and fixed/float-hybrids, as well as platforms specifically designed for media, communications and car infotainment. Most of their processors are available in multiple configurations, which means that at least the number of cores can be selected. TI DSPs very much rely on VLIW architectures with shared memory and cache hierarchies [58,59]. The processors may come equipped with special hardware to accelerate certain algorithms, e.g. FFT, and many peripheral interfaces.

The most powerful TI DSP, the c66x series, is similar in capability to ePUMA. Both support fixed- and floating-point operations and have somewhat similar instruction sets. The main differences are that the TI chip has hardware support for double-precision floating-point, whereas ePUMA has more powerful application-specific instructions. Furthermore, the c66x is a cache-based shared memory homogeneous architecture, while ePUMA is a heterogeneous distributed memory architecture.

Figure 6.1 shows a block diagram of the c66x DSP. One interesting thing to note is the fact that instruction fetching bandwidth is very high, which is necessary to support the wide VLIW instructions, although this is certainly not good from an energy perspective. Also, data cache bandwidth is quite low, half of datapath capability. In ePUMA the situation is the exact opposite. The ePUMA design has assumed that multiple data 


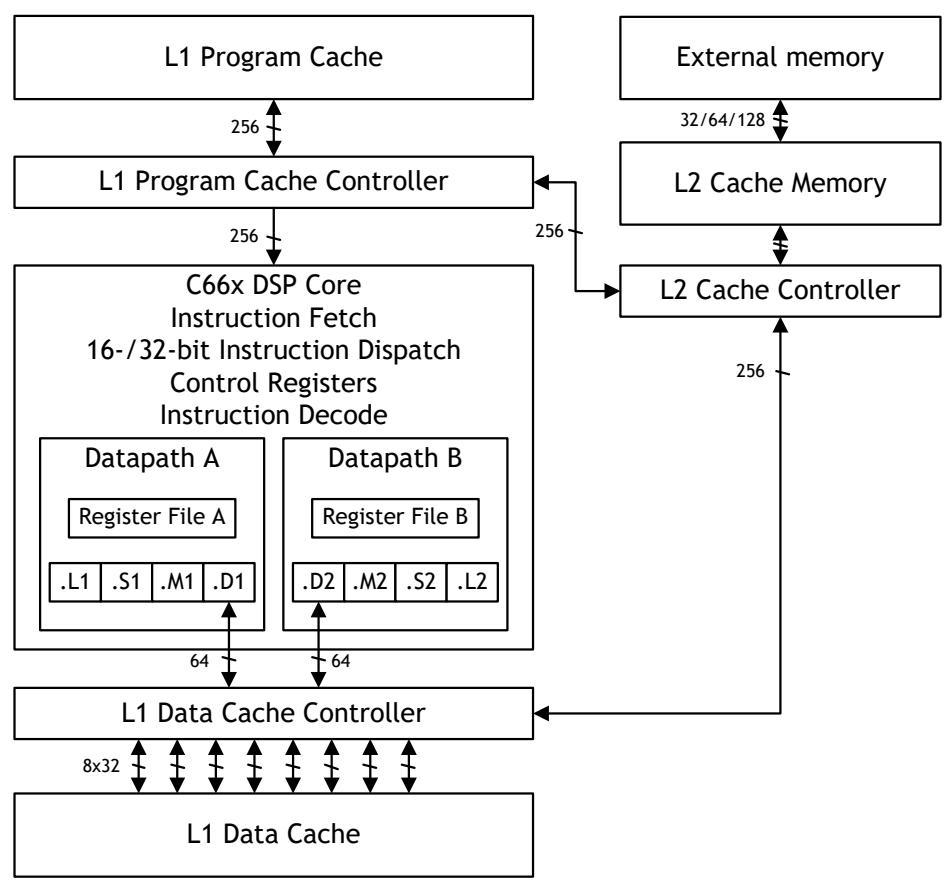

Figure 6.1: Texas Instruments c66x DSP architecture.

vectors could need to be accessed every cycle in order to avoid a memory bottleneck, and the architecture provides the necessary memory parallelism to cope with this situation. As we will see later, many TI DSP kernels are limited by exactly this fact. Only a few kernels are able to exploit the register file for data reuse as intended, simply because there is too little work to do per data element.

\subsection{CEVA DSPs}

CEVA sells DSP processor IPs for communications and multimedia. Like TI designs they are based on cache-based memory hierarchies and wide VLIW execution, extended with application-specific accelerators and peripherals. An example is shown in Figure 6.2. It depicts the XC4500 architecture, which is a multi-core architecture for wireless communi- 


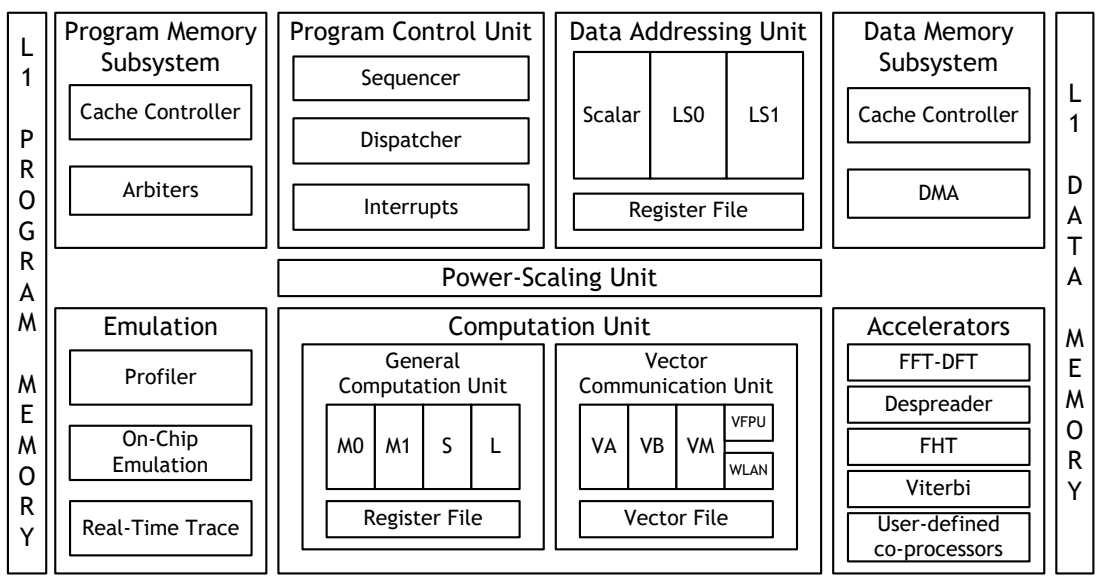

Figure 6.2: The CEVA XC4500 architecture.

cations. The computation unit is, unlike the TI design, divided into a general computation unit and a vector computation unit, which may improve energy efficiency. The architectures are otherwise quite similar, at least from a high-level perspective.

\subsection{NXP/Freescale DSPs}

Like TI and CEVA, NXP is another DSP company with similar offerings. Several designs are products from Freescale, which has since been bought by NXP. The DSPs are typically VLIW architectures with cachebased shared memory. The DSPs are programmed in C with inline assembly and like TI and CEVA, DSP libraries are provided for many critical kernels.

\subsection{Coresonic LeoCore}

The LeoCore architecture, shown in Figure 6.3, is a DSP architecture optimized for baseband processing $[9,60]$. Being a result of research at our research group, it is not surprisingly an inspiration to the work presented in this thesis. The basic design is similar to an ePUMA com- 


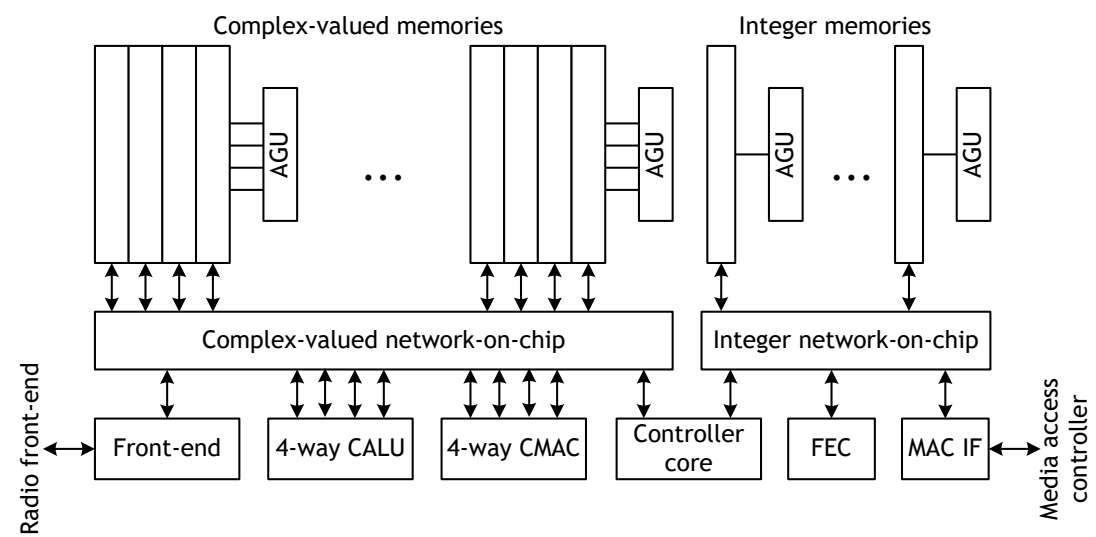

Figure 6.3: The LeoCore architecture.

pute cluster. A controller is responsible for managing execution and a set of complex-valued- and integer memories are connected to the execution units and accelerators using a heterogeneous network-on-chip. Each memory has its own AGU, which can be configured to access data according to some addressing pattern. The controller code may execute standard RISC instructions or send vector instructions to specialized vector execution units, such as a complex ALU and a complex MAC unit. This differs from the ePUMA design, where the CALU and CMAC corresponds to MPEs, which are complete processors with their own instruction flow. An additional difference is the generalized addressing capabilities of the MPE.

The LeoCore design is certainly efficient in its intended application domain, but may require modifications to work in other applications. That is one of the main goals of ePUMA, to expand the applicability of the architecture to as many applications as possible. ePUMA solves this by generalizing the instruction set and provide more flexible address generation. A potential problem with the LeoCore architecture is that a single source of instructions may not be enough to fill the execution units with work, if the assumption of long vector computing doesn't hold. This is one reason why the ePUMA MPE has its own instruction flow. As we will see later, the MPE also more aggressively targets the instruction delivery energy problem. To summarize, LeoCore may be 


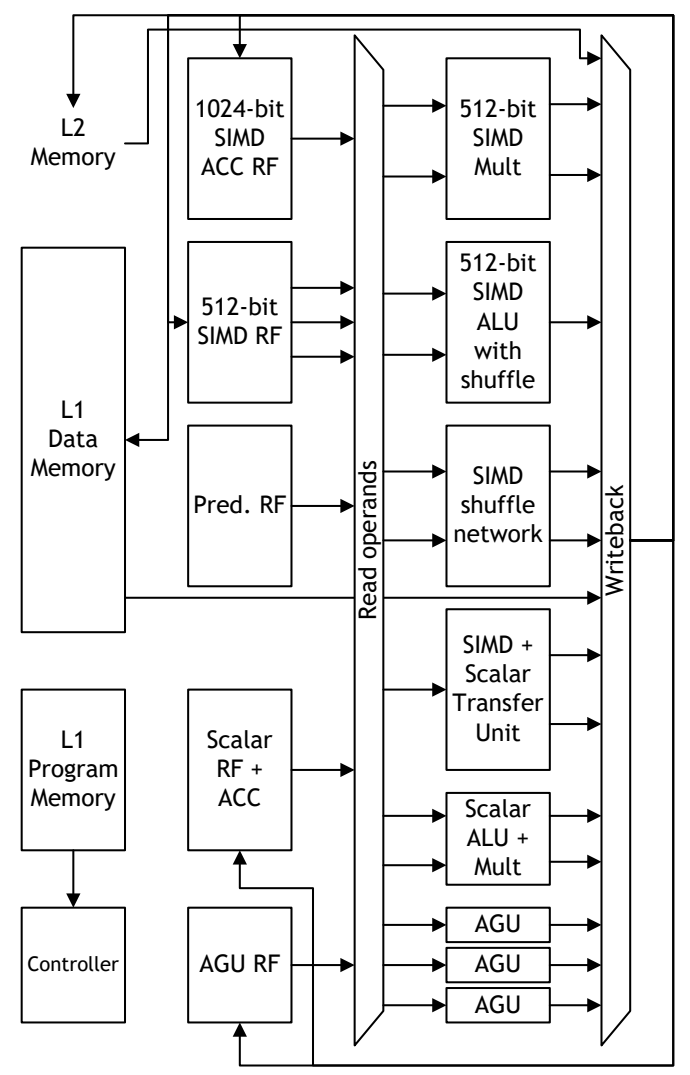

Figure 6.4: The Ardbeg architecture.

more energy efficient in the baseband processing domain, while ePUMA can support more applications without modifications.

\subsection{SODA and Ardbeg}

The research architecture SODA and its commercial improvement Ardbeg target wireless communications [61]. A schematic of Ardberg is shown in Figure 6.4. In some sense, SODA and Ardbeg are more similar to ePUMA than the previously mentioned architectures. One of the main ideas is to distribute the work between control processors and compute processors, where the compute processors use very wide vector pro- 


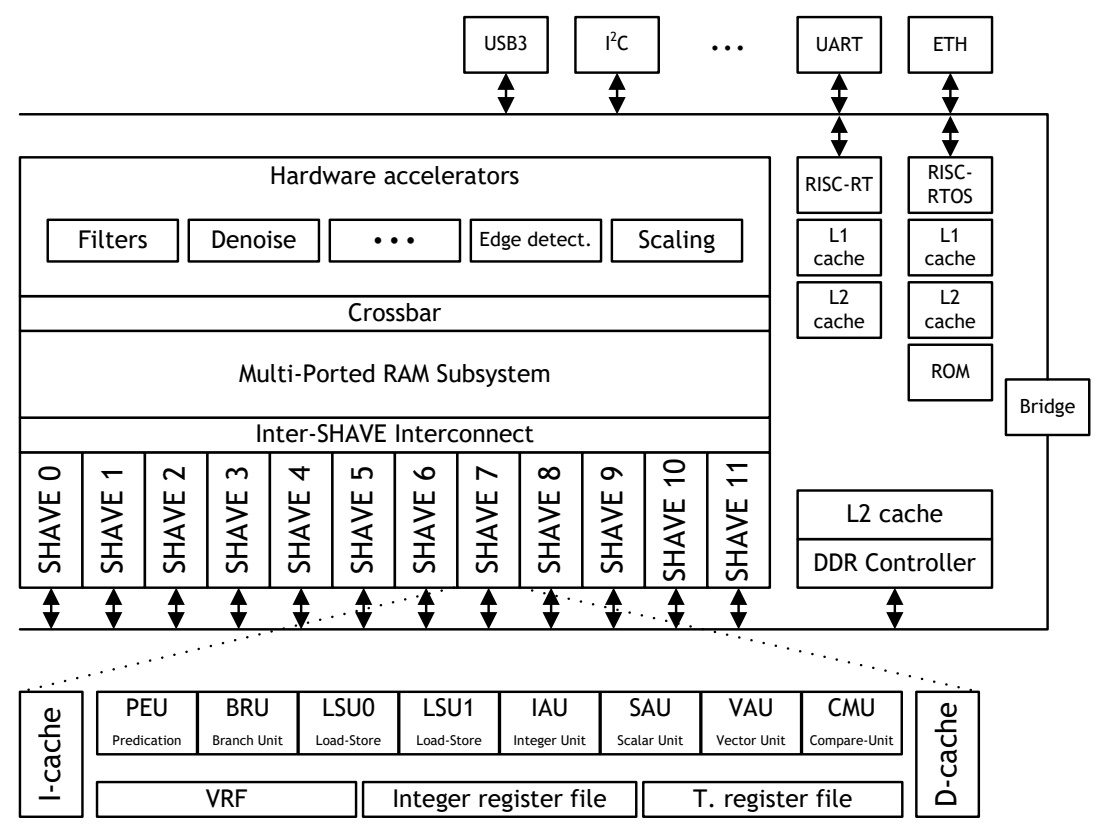

Figure 6.5: The Movidius Myriad 2 platform.

cessing. There are on the other hand several differences, such as somewhat limited and non-orthogonal vector data access, 2-way VLIW support (Ardbeg only) and no specific optimizations for instruction fetching energy reduction.

\subsection{Movidius Myriad 2}

Movidius produces DSPs for vision-related applications, such as smart cameras, robotics and security. One of their main products is the Myriad 2 Vision Processor Unit, shown in Figure 6.5. It contains 12 vector VLIW processors, imaging/vision accelerators, many peripheral interfaces, separate control processors and a cache-based memory hierarchy. 


\subsection{The ELM architecture}

The ELM research architecture addresses energy consumption in embedded processors [29]. It includes techniques such as instruction registers for reducing instruction fetching energy and explicit operand forwarding to reduce operand fetching energy. Still, even though instruction registers may reduce the energy consumed from instruction fetching, it doesn't reduce the dynamic energy consumed by control path switching.

\subsection{The MediaBreeze architecture}

The MediaBreeze multimedia processor introduces the concept of very wide instructions that are executed over many cycles, which may reduce both instruction fetching energy and control path switching [23]. An instruction can encode several levels of nested loops, conditional execution, computation and data access. The flexibility of a single instruction is very high, which results in a very large instruction word of up to 120 bytes. To implement the proposed functionality, the architecture decouples computation and data access and one of the main goals is to reduce the cost of supporting instructions for SIMD computing. The designers propose that this kind of design would be a useful addition to general-purpose processors, to improve performance and energy efficiency in multimedia applications.

\subsection{STI Cell Broadband Engine}

The STI Cell Broadband Engine is not designed to be a DSP processor, but it shares several features with ePUMA, such as a similar on-chip network and a similar offloading design [51]. Our own experiments show that the ePUMA design can be two orders of magnitude more energy efficient than Cell, while it sometimes may have similar or better performance, even on some non-typical DSP tasks [6]. This section might shed some light on this situation. 


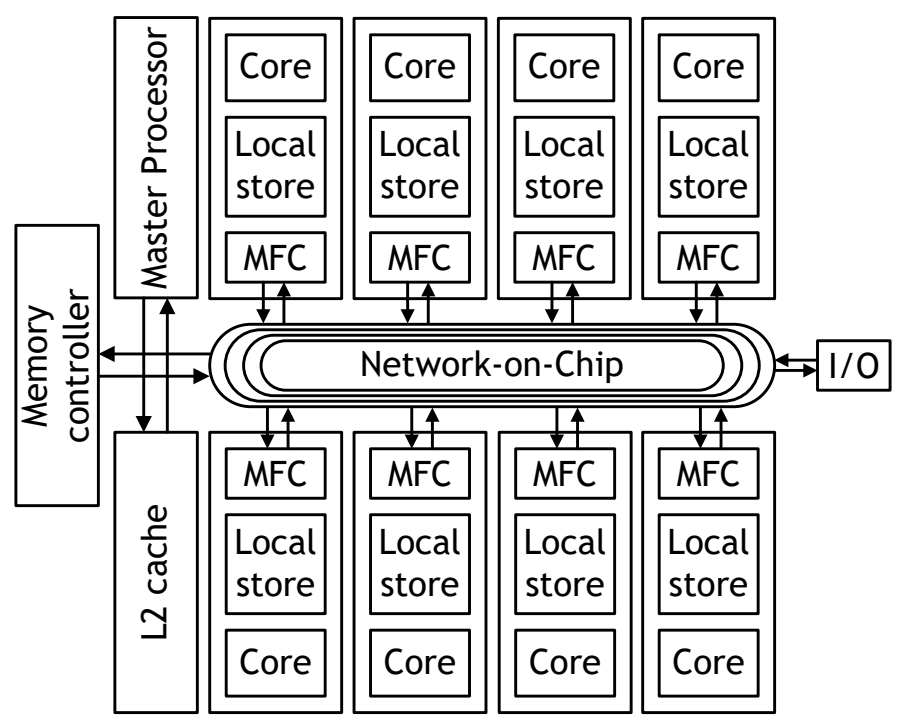

Figure 6.6: STI Cell architecture.

A block diagram of Cell is shown in Figure 6.6. The Cell processor is designed for a much higher clock frequency than ePUMA, which is certainly not beneficial from an energy perspective. Secondly, Cell lacks memory parallelism. Program code and data share a single memory and network communication, instruction fetching and operand fetching compete for the same resource. In addition, it may be hard to fully exploit the processor's execution units. For some kernels, $20 \%-40 \%$ of the processor cycles are wasted due to stalls because of branch instructions [62]. In the same experiment, an additional 10\%-34\% are wasted due to data dependencies and supporting instructions consume additional cycles, thus the ratio of compute cycles to total cycles is very low.

\subsection{1 x86, ARM and PowerPC}

The Intel x86 architecture is today the most commonly used processor for desktop PCs and servers. Likewise, the ARM architecture is the most commonly used application processor in handheld devices. Another ex- 
ample of a general-purpose processor architecture is the PowerPC. Processors based on these architectures are superscalar designs enhanced with one or multiple SIMD extensions, such as SSE, NEON and AltiVec [22, 21, 63]. Although ePUMA by no means is designed to be a direct competitor to these architectures, it is still interesting to compare the energy-efficiency of general-purpose designs with application-specific designs.

\subsection{Other platforms}

There are of course many more interesting platforms from manufacturers such as MediaTek, Qualcomm, Analog Devices and others. In many cases there is limited information about the exact details of their DSP subsystems and in this context it's best to avoid speculation about their designs.

\subsection{Summary}

This chapter reviewed alternatives to the ePUMA architecture, specifically other research- or commercial platforms and platform generators. ePUMA places itself in between the platform generators and the fixed systems, by providing some system configurability, but not at the level of detail of a processor generator. Processor generators are often constrained by some basic assumptions, which are strictly not necessary for compute-optimized DSPs. In contrast, commercial DSP platforms typically use a VLIW instruction set, which is certainly not that energy efficient, although it is possibly better than superscalar designs. There are some exceptions to this rule, such as the LeoCore and MediaBreeze architectures, but those architectures focus on specific applications. 


\section{Part III}

\section{Design of compute-optimized DSP co-processors}





\section{Chapter 7}

\section{Higher-order functional instructions}

The aim of many modern processor design approaches, such as superscalar and VLIW designs, is to increase the number of executed instructions per clock cycle (IPC). This makes sense, since a processor with higher IPC, and equally powerful instructions, will get more work done per time unit. The main drawback is the high energy cost of instruction delivery, as discussed in Section 2.5. At least in some computing domains, rather than increasing IPC, it makes much more sense to make the instructions more powerful. In fact, the best possible energy efficiency would be achieved if $I P C=0$ and the device still performs its task, since no energy would be spent on instructions. However, this would render the device completely non-programmable and the goal should instead be to seek a reasonable trade-off between programmability and energy efficiency that makes sense for the application domain.

This chapter will introduce the concept of higher-order functional (HOF) instructions, a concept that is excellently suited to implement many common DSP compute kernels. The name has been chosen due to its similarity with higher-order functions in functional programming languages such as F\# [64] and Haskell [65]. The idea is to increase the level of abstraction of the processor instruction set, by merging compute op- 
erations, data access and control into more complex instructions, to encode the most common computing patterns more efficiently and reduce the energy consumed from instruction delivery. Instead of operating on scalars in registers, or a fixed number of packed scalars in a SIMD register, we instead allow one- or multi-dimensional arrays of arbitrary size as operands and let the hardware handle the traversal of the data structures automatically. Such instructions may, depending on operand size, execute for hundreds or thousands of clock cycles, with only an initial instruction delivery cost and significantly reduced control path switching.

\subsection{Motivation}

The analysis of DSP kernels so far has revealed the fact that many kernels perform vector/matrix operations with predictable data access patterns. It is quite clear that SIMD or vector-style execution should be very beneficial in this scenario. Also, by implementing common operation subgraphs as hardware instructions, energy can be saved, by internalizing intermediate data to the processor's datapath and avoid explicit intermediate data storage [30]. If the instruction set is further specialized towards the target application, even more energy can be saved. Since the major hardware cost of a processor is memory area, the cost of adding application-specific instructions may be very small, but can improve performance considerably. In contrast, superscalar- and VLIW architectures increase arithmetic throughput using parallel execution units with relatively simple operations, which require a continuous stream of instruction input to be fully utilized. The flexibility of superscalar- and VLIW architectures is high, because the level of control over execution units is fine-grained, but as we shall see, it is often unnecessarily high for DSP kernels and it comes with a significant energy cost. This serves as our main motivation to find an alternative approach. 


\subsection{Goals}

The main goal of the research can be said to be the reduction of overhead energy. In principle, we would like that energy is only spent on datapath computing and the necessary memory operations to retrieve the compute data. At the same time we target high-performance DSP applications, which means that we must be able to meet high performance requirements. The efficient use of the available resources is essential.

To guide the processor development process and to be able to measure the success of all efforts, the following definition of the performance limit of an architecture may be used:

The performance limit of an architecture has been reached when all P-way binary datapaths consume $2 P$ data elements per clock cycle.

This definition implies that the bandwidth of all datapaths is fully utilized. The datapath may perform an arbitrarily complex function on its incoming data and we can search for opportunities for operation fusion to improve performance and energy efficiency. At the same time, the definition defines a relationship between data access performance and compute performance. If a $P$-way datapath can accept $2 P$ input elements per clock cycle, the data access capabilities of the processor should be able to provide exactly that, preferably regardless of the data access pattern. For this purpose, we must also seek a flexible enough memory subsystem that can cope with the most frequently used data access patterns in DSP, without any cycle time overhead.

\subsection{Computing patterns}

Higher-order functions define powerful patterns that can be used to express computation. These patterns are suitable for parallel processing on wide datapaths. The main purpose of classifying instructions into categories is to be able to determine desired hardware behavior. If a new application-specific instruction is added to the processor, it is enough to determine its pattern class, and a few additional parameters, so that the 


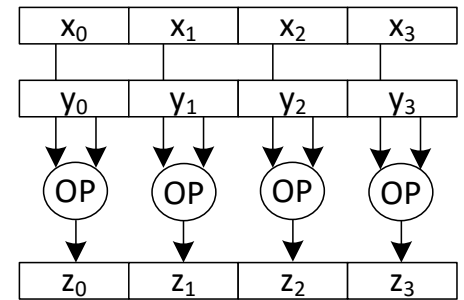

(a) map

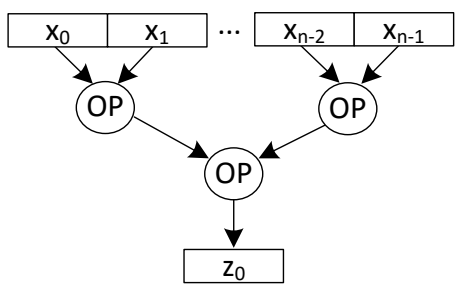

(b) reduce

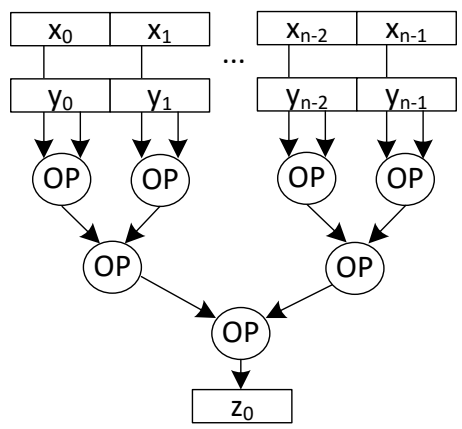

(c) map-reduce

Figure 7.1: The map, reduce and map-reduce patterns.

scheduling of events (such as memory writeback) and behavior (such as address generation behavior) can be determined. Our analysis of DSP kernels has identified the map, reduce and map-reduce patterns as the most important, patterns which will now be described.

\subsubsection{The map, reduce and map-reduce patterns}

The map pattern applies a function to every element in a list. As an example, a list of the elements $[1,3,-5,-4]$ and the function $a b s$ (absolute value), returns the list $[1,3,5,4]$. It may also accept multiple lists, in which case each function accepts the $i$ th element of all lists as input. Such a situation is shown in Figure 7.1(a). Any simple element-wise operation can be implemented with this pattern, such as additions and multiplica- 


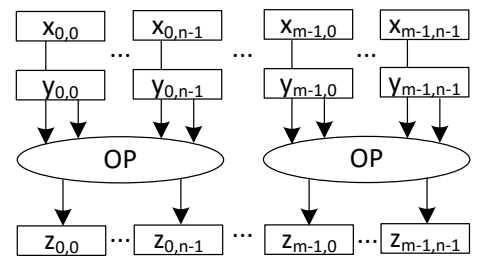

(a) block-map

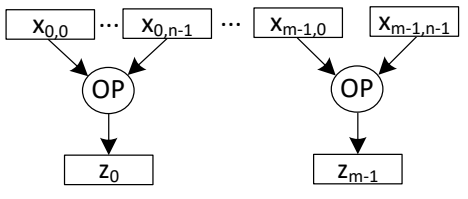

(b) block-reduce

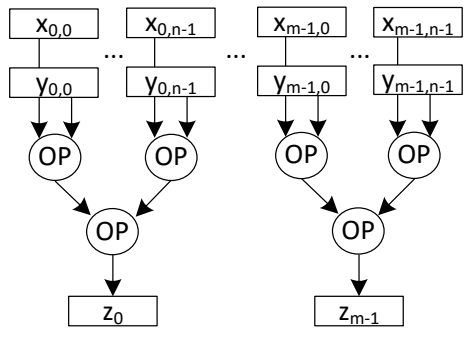

(c) block-map-reduce

Figure 7.2: The block-map, block-reduce and block-map-reduce patterns.

tions, as well as more complicated transcendental operations.

The reduce pattern reduces a list to a single value given some reduction operation, as shown in Figure 7.1(b). It can be used to implement operations such as the sum, minimum or maximum of a vector. The map-reduce pattern, shown in Figure 7.1(c), combines a map and a reduce operation. One of the most commonly used DSP operations, multiplication and accumulation, can be described with this pattern.

\subsubsection{Block patterns}

The map, reduce and map-reduce patterns can be generalized into block patterns, as exemplified in Figure 7.2(a), where the map pattern has been expanded into block-map. Instead of operating on single elements, each function operates on a block of data, which allows much more complex functions to be implemented. Examples of this include butterfly instructions for Fast Fourier Transform (FFT), special instructions for Discrete 
Cosine Transform (DCT) and instructions for sorting or prefix operations. Similarly, reduce and map-reduce may be expanded. In this context, blockreduce is defined as performing reductions of multiple blocks, whereas block-map-reduce is a combination of a map or block-map, followed by a block-reduce. These two patterns are shown in Figures 7.2(b) and 7.2(c). The block-reduce pattern can be used for computing the sum, min or max of each row / column of a matrix and block-map-reduce can implement an FIR-filter or convolution. The introduction of block patterns essentially introduces two computing dimensions to an instruction: the block size and the number of blocks. As we will see, this is very useful to produce compact code, as many DSP kernels are implemented with loop nests of two levels or more. There is however one practical implementation detail worth considering. The block-reduce and the reduction part of block-mapreduce are easily able to handle any block size and number of blocks by introducing one or a few accumulators. The number of elements in a block to block-map or the map part of block-map-reduce may instead be a fixed value defined by the datapath structure. A block size larger than $P$ will require storage of any number of intermediate results and may result in complex or expensive hardware.

In addition to code compaction, block-level patterns may also increase performance beyond traditional SIMD/vector computing. Consider a scalar datapath that is expanded into a $P$-way SIMD datapath through simple replication. The maximum possible relative speed-up naturally becomes $P$. However, the block-map pattern allows arbitrary functions of $P$ inputs (or $2 P$ inputs in case of two input lists) and possibly state, which allows the exploitation of both pipeline width and depth. As an example, consider the implementation of 16-point Fast Fourier Transform (FFT) on a $P$-way complex-valued datapath. A 1-way datapath could only perform a single complex multiplication per clock cycle, whereas a 2-way datapath (shown in Figure 7.3(a)) may perform both twiddle-factor pre-multiplication/scaling and a butterfly operation in a single step. A 4-way datapath, shown in Figure 7.3(b), can exploit an additional level of adders/subtractors, to complete a radix-4 butterfly. In 


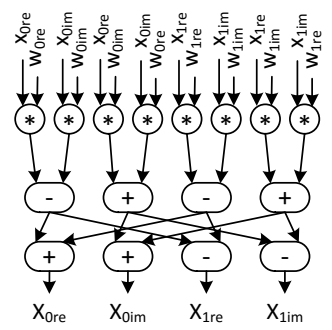

(a) Radix-2 butterfly.

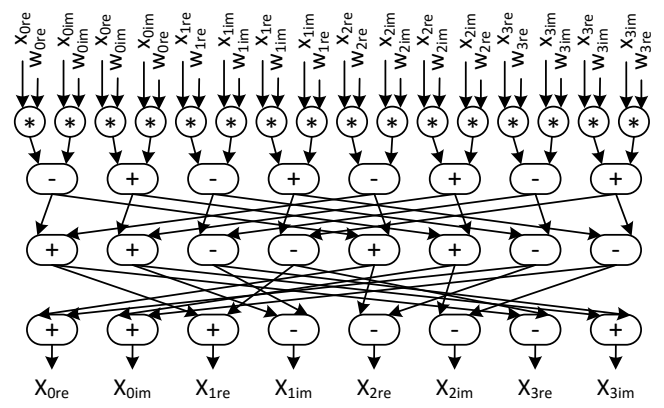

(b) Radix-4 butterfly

Figure 7.3: Implementations of radix-2- and radix-4 butterflies on 2- and 4-way complex datapaths.

Table 7.1: Theoretical performance of block-level $P$-way datapaths for Fast Fourier Transform.

\begin{tabular}{l|lcl}
\hline \hline $\mathbf{P}$ & Capability & Rel. speed-up & Pipeline depth \\
\hline 1 & 1 radix-2 BF/4 cycs & 1 & 1 mul, 1 add \\
2 & 1 radix-2 BF/cyc & 4 & 1 mul, 2 add \\
4 & 1 radix-4 BF/cyc & 16 & 1 mul, 3 add \\
16 & 1 FFT/cyc & 128 & 2 mul, 6 add \\
\hline \hline
\end{tabular}

the extreme case, a 16-way datapath could reach the throughput of one 16-point FFT per clock cycle.

The relative theoretical performance of the different FFT-specialized datapaths is shown in Table 7.1. This analysis ignores potential data access overhead, pipeline effects and possible optimizations. The performance clearly scales faster than $P$, but on the other hand, a larger datapath has a higher cost and other instructions may not benefit from a large pipeline depth, which may lead to limited hardware reuse. 


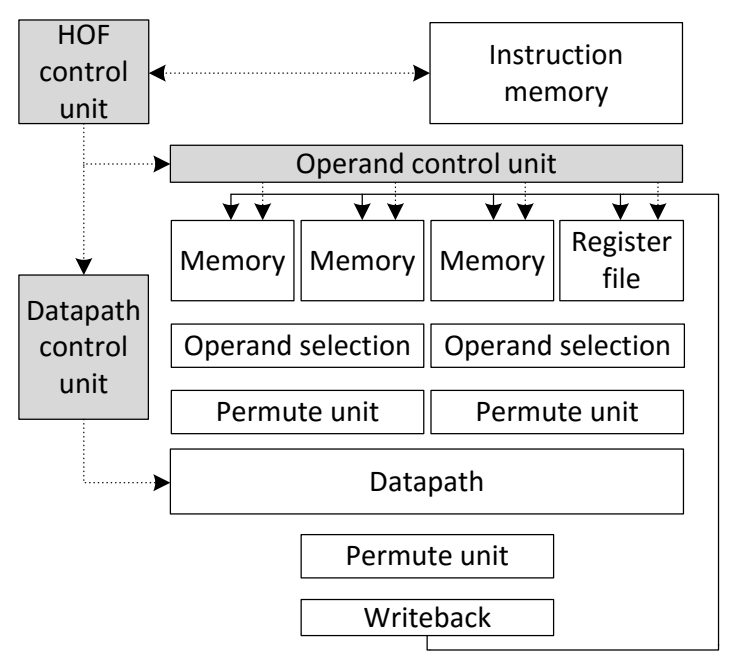

Figure 7.4: High-level organization of processor pipeline to execute HOF instructions.

\subsection{Data access}

The introduction of the previously described computing patterns suggests that an instruction could traverse large quantities of data, quantities so large that register file storage may not be appropriate. Furthermore, many kernels require out-of-order data access. To reach the computing limit of a parallel datapath and fully utilize the potential for long vector computing, it should be possible to perform out-of-order data access to memory without performance penalty. A possible pipeline organization with parallel processing and parallel memory is shown in Figure 7.4. In this structure, data access is fully orthogonal to computing. The problem is then simply to find an appropriate datapath for the application and a general enough data access path that supports the required access patterns. Note that this model does not exclude the use of a register file. A register file will be useful for fast access to variables. It may even hold frequently accessed shorter vectors for energy reduction purposes, since register file accesses may consume less energy than memory accesses. 


\subsection{The higher-order functional instruction}

The general (two-dimensional) higher-order functional instruction proposed by this work may be described by the following pseudo-assembly syntax:

$$
N_{o} * \text { operation }\left[N_{i}\right] \text { dst } \operatorname{src} 0 \quad \operatorname{src} 1
$$

where operation defines the datapath operation, $N_{i}$ is the inner computing dimension (number of operations per block), $N_{o}$ is the outer computing dimension (number of blocks), and $d s t, \operatorname{src} 0$ and $\operatorname{src} 1$ are the destination and source operands respectively. The set of available operations (or functions) is defined by the instruction set, which may be of any of the previously described patterns.

We will now analyze the performance limit of a processor implementing HOF instructions. Consider the implementation of HOF instructions with the proposed patterns using a single parallel datapath of processing width $P$. The minimum cycle time $t_{\geq c y c}$ of an instruction is

$$
t_{\geq c y c}=\left\lceil N_{o} N_{i} / P\right\rceil
$$

The datapath and data access hardware may become extremely complex to achieve this cycle time, since the processing of multiple blocks may have to be overlapped in time, especially when $P$ and $N_{i}$ are relatively prime. If we constrain the implementation so that two blocks may not be executed at the same time on the datapath, that is we completely issue the processing of one block of $N_{i}$ elements before we may start issuing data for the next block. The lower bound then becomes

$$
t_{\geq c y c}=N_{o}\left\lceil N_{i} / P\right\rceil
$$

This cycle time can only be reached if operands can be delivered at a high enough pace, which we model using the effective operand delivery bandwidth $P_{o p}$. As an example, the binary map pattern requires $3 N$ read/write operations, which places stringent constraints on data access. The 
lower cycle time bound then becomes:

$$
\begin{aligned}
\text { Unary map }: t_{\geq c y c} & =\max \left(N_{o}\left\lceil N_{i} / P\right\rceil,\left\lceil N_{o}\left(2 N_{i}\right) / P_{o p}\right\rceil\right) \\
\text { Binary map }: t_{\geq c y c} & =\max \left(N_{o}\left\lceil N_{i} / P\right\rceil,\left\lceil N_{o}\left(3 N_{i}\right) / P_{o p}\right\rceil\right) \\
\text { Reduce }: t_{\geq c y c} & =\max \left(N_{o}\left\lceil N_{i} / P\right\rceil\left\lceil N_{o}\left(N_{i}+1\right) / P_{o p}\right\rceil\right) \\
\text { Unary map-reduce }: t_{\geq c y c} & =\max \left(N_{o}\left\lceil N_{i} / P\right\rceil,\left\lceil N_{o}\left(N_{i}+1\right) / P_{o p}\right\rceil\right) \\
\text { Binary map-reduce }: t_{\geq c y c} & =\max \left(N_{o}\left\lceil N_{i} / P\right\rceil,\left\lceil N_{o}\left(2 N_{i}+1\right) / P_{o p}\right\rceil\right)
\end{aligned}
$$

Note that although it may be impractical to overlap different blocks in time on the same datapath, the same may not be true for data access. The actual value of $P_{o p}$ depends on several factors and may not even be an integer. Naturally it depends on the available operand delivery bandwidth of the hardware, but it may also depend on processor pipeline organization, the data access pattern and how the data has been distributed over memory or register file resources. As an example, if both source lists and the destination list to binary map are placed in the same single-ported memory, actual compute performance will be limited to $1 / 3$ of datapath capability because of memory conflicts. The capabilities for operand access is very important to avoid operand delivery bottlenecks, and will be further discussed in Chapters 8 and 9.

The HOF concept does not exclude the use of multiple datapaths. Instead of a single $P$-way datapath, we can use $P_{d p} P$-way datapaths to process multiple blocks simultaneously. If data access is ignored, the lower limit on cycle time is reduced to

$$
t_{\geq c y c}=\left\lceil N_{o} / P_{d p}\right\rceil\left\lceil N_{i} / P\right\rceil
$$

The advantage is that higher performance can be achieved. On the other hand it increases the data access load, further restricts memory address generation and may leave datapaths unused for one-dimensional instructions. 
Listing 7.1: Pseudocode for vector addition.

Listing 7.2: Possible pseudo-assembly implementation. In this context ++ represents consecutive addressing of elements.

1 add[N] $\mathrm{C}++\mathrm{a}++\mathrm{b}++$

\subsection{Kernel examples}

This section will exemplify the use of HOF instructions, to demonstrate simple kernel implementation and analyze kernel performance. We derive lower bounds on cycle time and also lower bounds on cycle time considering a pipelined processor implementation, disregarding possible data access bottlenecks. The goal of the processor implementation is to approach these lower bounds and they help us to measure our success.

\subsubsection{Element-wise operations}

Consider the code in Listing 7.1, which represents element-wise vector addition. This can be implemented in pseudo-assembly as shown in Listing 7.2 and the theoretical lower bound on cycle time using a single $P$-way datapath is defined by Equation 7.4. Considering a pipelined processor implementation, the lower cycle time bound is

$$
t_{\geq c y c, p i p e}=\lceil N / P\rceil+L_{\text {pipe }}
$$

where $L_{\text {pipe }}$ represents the fact that when a processor is pipelined, there will be at least a certain pipeline delay before the result is actually written to the destination. This delay may be hidden by issuing other unrelated instructions.

In principle, any element-wise operation can be implemented in this way. Operations that require more than one cycle per element to com- 
Listing 7.3: Pseudocode for 1D-convolution. The coefficients vector have been reversed and edge cases are ignored for simplicity.

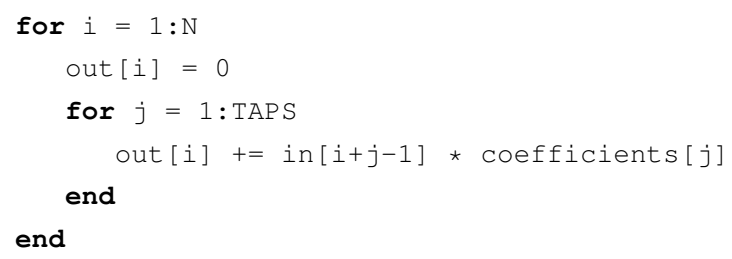

plete are also covered and the performance can be modeled by a corresponding reduction of datapath parallelism $P$. In addition it should be noted that the data structure to be processed does not have to be a vector of consecutive elements. An $m \times n$ matrix where the rows/columns are stored consecutively may be processed with the same instruction if $N=m \cdot n$. Non-consecutive data access, on the other hand, requires special care and will be discussed later.

\subsubsection{Convolution and FIR filtering}

Consider the code in Listing 7.3, which represents a high-level pseudocode implementation of a very frequently executed computing pattern: 1D convolution. This code may be translated into pseudo-functional style, as shown in Listing 7.4. It uses repeated map-reduce with multiplication and addition respectively, which corresponds to multiply-andaccumulate (MAC) or a dot product. If the datapath provides a block-mapreduce-based multiply-and-accumulate reduction instruction, here called tmac, the assembly code may look like in Listing 7.5. A lower bound on pipelined cycle time is

$$
t_{\geq c y c, p i p e}=N\lceil T A P S / P\rceil+L_{\text {pipe }}
$$


Listing 7.4: $1 \mathrm{D}$ convolution in a pseudo-functional style.

Listing 7.5: $1 \mathrm{D}$ convolution in a pseudo-assembly. ++ represents incremental addressing, \% represents modulo addressing and values within parenthesis represent the pointer modification to perform after each inner dimension has been processed.

\subsubsection{Fast Fourier Transform}

The Fast Fourier Transform (FFT) is best implemented as a set of processing layers [66]. For simplicity we only consider FFTs where the number of points $N=4^{L}$, which can be implemented with $L$ complex radix-4 butterfly layers. A possible pseudo-assembly implementation is shown in Listing 7.6. The lower bound on cycle time is

$$
t_{\geq c y c}=L \cdot\lceil(N / 4) / P\rceil
$$

where $P$ in this case represents the number of complex radix- 4 butterflies the datapath can process per cycle. However, if we consider a pipelined implementation, there is a data dependency between butterfly layers and we can expect that there will be some delay $L_{w r}$ before we can start reading input for a subsequent layer, either because of an actual data dependency or because of a resource conflict (such as the data being stored in a single-ported memory). A lower bound on cycle time considering the pipeline can then be calculated as

$$
t_{\geq c y c, p i p e}=L \cdot\left(\lceil(N / 4) / P\rceil+L_{w r}\right)-L_{w r}+L_{\text {pipe }}
$$

Note that there is no data dependency after the final layer, but instead we may expect some latency before the pipeline is empty. 
Listing 7.6: Pseudo-assembly implementation of $4^{L}$ FFT. The $c r 4 b f$ instruction represents a complex radix-4 butterfly instruction.

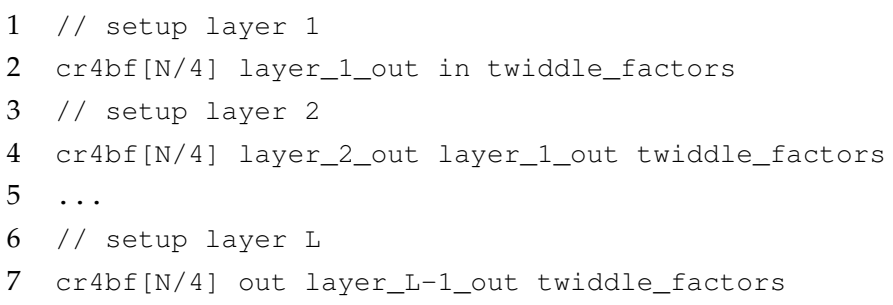

It may be of interest to estimate the actual cycle time. Each layer may require some setup code, which takes at least $L_{\text {setup }}$ cycles to execute. This overhead may be hard to hide for the first layer. However, for subsequent layers, the setup code may be overlapped with the write-readdelay $L_{w r}$, at least if resource conflicts can be avoided. In this case we can estimate the actual cycle time as

$$
\left.t_{c y c} \approx L_{\text {setup }}+L\lceil(N / 4) / P\rceil+(L-1) \cdot \max \left(L_{\text {setup }}, L_{w r}\right)\right)+L_{\text {pipe }}
$$

\subsection{Related instruction sets and architectures}

This section will compare the HOF instruction set architecture concept with related work from the commercial market and academia.

\subsubsection{VLIW and superscalar architectures}

VLIW and superscalar architectures have high flexibility and may have relatively high performance because of parallel execution of fine-grained instructions. Their drawbacks include relatively high energy consumption, large code size, limited scalability, high instruction delivery requirements and high register file pressure. One distinct advantage is the relatively mature compiler technology for these architectures, although the results may be far from perfect. 
Generating high performance code for these architectures may be far from trivial. In the DSP domain, the critical part is typically loop nests, which have to be unrolled and scheduled carefully [67]. Listing 7.7 shows an implementation of a real-valued dot product on a commercial VLIW-DSP processor. Unrolling and software pipelining is used to fill eight parallel execution units with work. The code becomes large and complex. With an unroll factor of 2, it only accepts vectors with an even number of elements. Also, the code doesn't implement an epilogue, which means that the main loop will continue reading unnecessary data, which in the worst case could lead to cache misses. A similar dot product implementation which contains an epilogue is almost twice as long. A HOF version is shown in Listing 7.8. It accepts an arbitrary number of elements, doesn't perform any unnecessary memory operations and requires only a single instruction.

Many superscalar and VLIW architectures are so called load-store architectures, which means that memory is always accessed via load/store instructions and compute instructions can only operate on registers. This is not required for a HOF architecture. The HOF concept promotes the use of complex instructions, to improve performance and reduce instruction delivery. Relatively speaking, VLIW and superscalar architectures only implement simpler instructions. There are of course exceptions to these rules, where CISC architectures such as the Intel x86 can both operate directly on memory and implements complex instructions, such as transcendental operations [22].

\subsubsection{SIMD and vector architectures}

SIMD and vector architectures, including also VLIW and superscalar architectures enhanced with SIMD instructions, have some features in common with the HOF concept. However, HOF instructions are designed to be more self-sufficient, because they pack control and data access information with the compute operation. Typical SIMD/vector instructions that perform the actual computations must be aided by load and store instructions for doing memory to register transfers, use explicit 
Listing 7.7: A possible implementation of dot product on a VLIW processor [68].

\begin{tabular}{|c|c|c|c|c|c|}
\hline 1 & & & LDW & . D1 & $\star \mathrm{A} 4++, \mathrm{A} 2$ \\
\hline 2 & 11 & & LDW & . D2 & $\star B 4++, B 2$ \\
\hline 3 & || & & MVK &. $\mathrm{S} 1$ & $50, \mathrm{~A} 1$ \\
\hline 4 & $1 \mid$ & & ZERO & . L1 & A 7 \\
\hline 5 & | | & & ZERO &. $\mathrm{L} 2$ & B7 \\
\hline 6 & & {$[\mathrm{~A} 1]$} & SUB &. $\mathrm{S} 1$ & $\mathrm{~A} 1,1, \mathrm{~A} 1$ \\
\hline 7 & | | & & LDW & . D1 & $\star \mathrm{A} 4++, \mathrm{A} 2$ \\
\hline 8 & || & & LDW &. $\mathrm{D} 2$ & $\star \mathrm{B} 4++, \mathrm{B} 2$ \\
\hline 9 & & [A1 ] & SUB &. $\mathrm{S} 1$ & $\mathrm{~A} 1,1, \mathrm{~A} 1$ \\
\hline 10 & | | & {$[\mathrm{A} 1]$} & B &. $\mathrm{S} 2$ & LOOP \\
\hline 11 & || & & LDW & . D1 & $\star \mathrm{A} 4++, \mathrm{A} 2$ \\
\hline 12 & || & & LDW &. $\mathrm{D} 2$ & $\star \mathrm{B} 4++, \mathrm{B} 2$ \\
\hline 13 & & {$[\mathrm{~A} 1]$} & SUB &. $\mathrm{S} 1$ & $\mathrm{~A} 1,1, \mathrm{~A} 1$ \\
\hline 14 & 11 & {$[\mathrm{~A} 1]$} & B &. $\mathrm{S} 2$ & LOOP \\
\hline 15 & || & & LDW & . D1 & $\star \mathrm{A} 4++, \mathrm{A} 2$ \\
\hline 16 & $1 \mid$ & & LDW &. $\mathrm{D} 2$ & $\star \mathrm{B} 4++, \mathrm{B} 2$ \\
\hline 17 & & [A1 ] & SUB &. $\mathrm{S} 1$ & $\mathrm{~A} 1,1, \mathrm{~A} 1$ \\
\hline 18 & | | & {$[\mathrm{A} 1]$} & B & .52 & LOOP \\
\hline 19 & || & & LDW & . D1 & $\star \mathrm{A} 4++, \mathrm{A} 2$ \\
\hline 20 & 11 & & LDW &. $\mathrm{D} 2$ & $\star \mathrm{B} 4++, \mathrm{B} 2$ \\
\hline 21 & & & MPY & . M1X & $\mathrm{A} 2, \mathrm{~B} 2, \mathrm{~A} 6$ \\
\hline 22 & 11 & & MPYH & . M2X & $\mathrm{A} 2, \mathrm{~B} 2, \mathrm{~B} 6$ \\
\hline 23 & 11 & {$[\mathrm{~A} 1]$} & SUB &. $\mathrm{S} 1$ & $\mathrm{~A} 1,1, \mathrm{~A} 1$ \\
\hline 24 & 11 & {$[\mathrm{~A} 1]$} & B &. $\mathrm{S} 2$ & LOOP \\
\hline 25 & 11 & & LDW &. $\mathrm{D} 1$ & $\star \mathrm{A} 4++, \mathrm{A} 2$ \\
\hline 26 & | | & & LDW &. $\mathrm{D} 2$ & $\star \mathrm{B} 4++, \mathrm{B} 2$ \\
\hline 27 & & & MPY & . M1X & $\mathrm{A} 2, \mathrm{~B} 2, \mathrm{~A} 6$ \\
\hline 28 & $\mid 1$ & & MPYH & . M2X & $\mathrm{A} 2, \mathrm{~B} 2, \mathrm{~B} 6$ \\
\hline 29 & 11 & {$[\mathrm{~A} 1]$} & SUB &. $\mathrm{S} 1$ & $\mathrm{~A} 1,1, \mathrm{~A} 1$ \\
\hline 30 & | | & {$[\mathrm{A} 1]$} & B &. $\mathrm{S} 2$ & LOOP \\
\hline 31 & 11 & & LDW &. $\mathrm{D} 1$ & $\star \mathrm{A} 4++, \mathrm{A} 2$ \\
\hline 32 & $\mid 1$ & & LDW &. $\mathrm{D} 2$ & $\star \mathrm{B} 4++, \mathrm{B} 2$ \\
\hline 33 & LOO & P : & & & \\
\hline 34 & & & $\mathrm{ADD}$ & . L1 & $\mathrm{A} 6, \mathrm{~A} 7, \mathrm{~A} 7$ \\
\hline 35 & 11 & & $\mathrm{ADD}$ &. $\mathrm{L} 2$ & $B 6, B 7, B 7$ \\
\hline 36 & | | & & MPY & . M1X & $\mathrm{A} 2, \mathrm{~B} 2, \mathrm{~A} 6$ \\
\hline 37 & 11 & & MPYH & . M2X & $\mathrm{A} 2, \mathrm{~B} 2, \mathrm{~B} 6$ \\
\hline 38 & 11 & {$[\mathrm{~A} 1]$} & SUB &. $\mathrm{S} 1$ & $\mathrm{~A} 1,1, \mathrm{~A} 1$ \\
\hline 39 & $\mid 1$ & {$[\mathrm{~A} 1]$} & B &. $\mathrm{S} 2$ & LOOP \\
\hline 40 & | | & & LDW &. $\mathrm{D} 1$ & $\star \mathrm{A} 4++, \mathrm{A} 2$ \\
\hline 41 & 11 & & LDW &. $\mathrm{D} 2$ & $\star \mathrm{B} 4++, \mathrm{B} 2$ \\
\hline 42 & & & $\mathrm{ADD}$ &. $\operatorname{L1X}$ & $\mathrm{A} 7, \mathrm{~B} 7, \mathrm{~A} 4$ \\
\hline
\end{tabular}


Listing 7.8: An example of a HOF implementation of dot product.

counters and conditional jump instructions to implement iteration and memory addressing may have to be performed separately. Rearrangement of data may have to be performed with time-consuming shuffle instructions $[22,51]$. If the number of elements to be processed is not a multiple of SIMD width, special code to handle such cases may have to be added. Many SIMD architectures put additional restrictions on memory access, such as alignment requirements. Even something as simple as scalar to vector expansion may take additional time. Because of these restrictions it may be hard to reach the relative speedup of the processing width in practice.

\subsubsection{Application-specific architectures}

For specific application domains there are instruction set architectures similar to the HOF concept, such as the already mentioned LeoCore architecture [60]. This architecture can also process e.g. an FFT layer with a single instruction and operates directly on memory. The differences include the two-dimensionality of HOF instructions and the generalized addressing, which will be discussed later. There are also other differences, which are not related to the instruction set. Similarly, the MediaBreeze architecture implements very wide multi-cycle instructions for media applications [69]. The instructions pack even more information than a HOF instruction, but are targeted toward media only. The idea of HOF instructions is to support a wide variety of applications, at least applications that perform vector/matrix processing.

Some architectures focus on specific types of operations, instead of specific applications. One example is architectures for matrix operations or linear algebra $[70,71,72]$. The main goal is typically not energy reduction, but instead to improve performance by using specialized datapaths. These architectures may not even use multi-cycle instructions, 
even though it would be possible. The HOF concept covers matrix operations and linear algebra, but also many other operations and aims both for high performance and high energy efficiency.

\subsubsection{Comparative summary}

To summarize the advantages of HOF instructions, compared to the other mentioned approaches, the following can be said:

- Possibility for better hardware utilization and performance: Once a HOF instruction is running, it may execute over many cycles. The maximum utilization of the hardware is then handled by the hardware designer, rather than the compiler or programmer, which can result in higher performance.

- More complex operations: The hardware designer may implement an arbitrarily complex datapath to accelerate any given function. This is certainly possible for the other types of architectures as well, but the traditional approach is simply to replicate datapaths, or introduce different datapaths for different kinds of operations, which is more limited in terms of performance scalability.

- High performance for multiple-short-vector processing: Because of the two-dimensional HOF computing patterns, data access and control bottlenecks may be avoided even for processing of short vectors. If source- and destination operand addressing is flexible enough, it may be possible to describe multiple short vectors operations with a single HOF instruction. In contrast, a SIMD or 1Dvector architecture may instead spend more time on the supporting work (e.g. setting address pointers) than the actual compute work.

- Smaller code size: The written or generated assembly code is compact to read and the final binary may be small.

- Less program code movement: A smaller kernel binary means that less kernel code has to be loaded from off-chip memory. This saves 
both time and energy. In addition, if the kernels are small, the onchip memories may hold more kernels, which results in less frequent kernel code reloads.

- Less control path switching: Dynamic energy consumption in the processor's control path can be reduced. An instruction executing over several cycles may keep most control signals stable for its duration.

- Less instruction fetching: If instructions execute over many cycles, the instruction memory is less frequently used. This reduces energy consumption and/or frees up the instruction memory to be used for other purposes.

- Less intermediate data storage: Register file pressure may be significantly reduced. When more complex instructions are implemented, many intermediate results are simply flowing through the datapath, rather than requiring explicit register file storage.

- Processing width independence: In contrast to typical SIMD architectures, the HOF concept does not explicitly expose the width of the datapath, meaning that the datapath width could be modified without program modification. The hardware may also transparently handle vectors of lengths other than multiples of datapath width. Depending on the actual hardware implementation, some restrictions may apply.

- Higher level of abstraction: Higher-order functional instructions can hide architecture complexity and increase the level of abstraction of hardware instructions. It provides more powerful building blocks for the DSP programmer and compiler.

- Hardware support for higher-order functional programming: High-level code written using higher-order functions may have direct equivalents in hardware. The translation to machine code may be simplified. 
Although it would be theoretically possible to issue multiple HOF instructions over multiple datapaths, a single HOF instruction already encodes lots of parallelism. If we constrain the architecture to only issue maximally a single instruction per clock cycle, instruction scheduling is simplified. Compared to VLIW and superscalar architectures, the hardware or compiler does not have to consider the effects of parallel instruction issuing and we can still achieve high performance.

There are of course several disadvantages of the proposed instructions, including:

- The HOF concept is not helpful for non-vector computing.

- The pipeline may be longer, due to a larger datapath depth which may also be put in series with memory accesses. This may reduce the performance of code with data dependent decision making.

- The instruction word may be long to encode all flexibility.

- The processor may not be suitable to execute complex control flow.

- The processor becomes more vulnerable to appropriate instruction set selection.

Many of these disadvantages could be troublesome in general, but are much less so in the DSP domain. First of all, vector computing is the norm and many kernels are extremely predictable. The disadvantage of a long individual instruction word will be offset by much fewer instructions. As we shall see later, a practical HOF implementation may still have shorter instruction words than a VLIW processor. It is certainly true that a HOF-based processor may execute general control flow slower than a VLIW- or superscalar processor. However, a pure HOF-based processor is possibly only used as a co-processor to a second processor that executes the control flow. The most critical part then becomes instruction set selection. 


\subsection{Summary}

This chapter introduced the concept of higher-order functional instructions and demonstrated how they may implement common DSP kernels. Although the concept may not be useful for general code, it addresses the computing patterns typically found in DSP kernels and can potentially reduce the energy consumption from instruction delivery and control path switching significantly. The savings will be quantified in the later chapters. 


\section{Chapter 8 \\ Local memory architecture}

In order to approach the performance limit of a $P$-way binary datapath, and fully unlock the performance potential of HOF instructions, we've already identified that we could need a local memory bandwidth of up to $3 P$ elements per clock cycle, considering both two source operands and one destination operand. This bandwidth may have to be sustained over thousands of cycles. Additionally, many algorithms require out-oforder access to data, which many traditional SIMD/vector architectures do not handle without a loss of performance. If this performance bottleneck is to be eliminated, data access and computing must be made fully orthogonal, which means that data access cost is hidden behind computing. The goal of this chapter is to determine the desired properties of a memory subsystem and its address generation capabilities, in order to be able to handle the most commonly occurring access patterns efficiently.

\subsection{Memory subsystem requirements}

A memory subsystem designed for a specific application could be designed from the exact known addressing patterns. However, to support general addressing patterns, the memory subsystem must be able to support the following:

- Selection: The memory subsystem must be able to select some (or 
all) elements from some data set for processing on the datapath (up to $3 P$ elements per cycle).

- Duplication: The memory subsystem must be able to duplicate selected elements. A common data access operation is to duplicate a scalar to all inputs of the datapath.

- Permutation: It should be possible to rearrange the elements to some other order than memory storage order. This is especially important for e.g. block-map operations, since all datapath inputs may not be equivalent.

For fully flexibility, these operations must be supported for both readand write-operations.

A common memory structure for SIMD processors is shown in Figure 8.1(a). Each memory word stores $P$ elements and only a single vector may be accessed per cycle. Full performance is only achieved for aligned consecutive data. In other cases, the data has to be prepared for parallel processing, which is typically performed in some temporary storage, such as a register file. The same memory could be equipped with multiple access ports, but this solution has a high hardware cost.

A more flexible solution is shown in Figure 8.1(b). The memory has been divided into $P$ parallel memory banks and any memory access pattern that doesn't cause multiple accesses to the same memory bank can be serviced without any loss of performance. This structure may also be multi-ported, as shown in Figure 8.1(c), with $P_{\text {ports }}$ ports per memory bank.

If $P_{\text {ports }}=P$, we can support any addressing pattern without performance penalty. The main problem is that the memory cost scales roughly linearly with $P_{\text {ports }}$, but the amount of memory stays the same. Furthermore, memories with many access ports may not be available in practice. Multi-bank memory is often sufficient and this approach is nowadays popular in both DSP processors [58] and GPUs [73]. Especially if the data access patterns are statically known, the data sets can be prepared for conflict-free parallel memory access under memory bank constraints, 


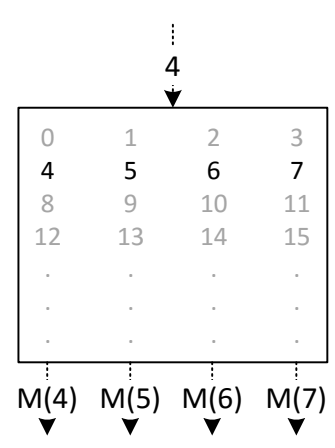

(a) Single-bank.

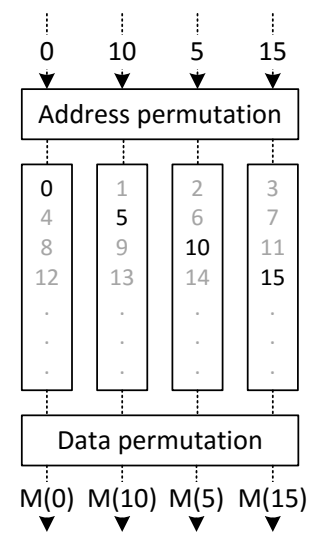

(b) Multi-bank.

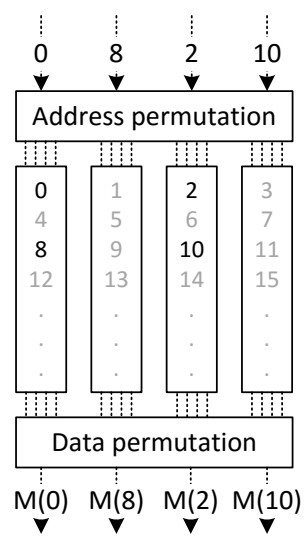

(c) Multi-port.

Figure 8.1: Memory structures for parallel data access.

to guarantee conflict-free access or a minimal number of conflicts.

Most often we consider the three instruction operands to be disjoint. This means that the worst-case required bandwidth of $3 P$ elements per clock cycle can be regarded as three separate accesses, each of $P$ elements. If this is not the case, it may be possible to split source and destination buffers and use ping-pong buffering. This assumption eases the memory subsystem requirements, as multiple concurrent reads/writes to the same location would require multi-port memory.

Table 8.1 compares the three different memory organizations for $P$ way parallel vector operand access. All three organizations perform equally well for aligned consecutive addressing, however the single-bank memory performs far worse for unaligned and out-of-order accesses. Multi-banking however has a certain cost, which we model with the relative cost factor $C_{M B}$. The actual value of $C_{M B}$ depends on several factors and will be discussed in Section 8.2.2. The performance gained by using multi-bank memory has been demonstrated in many other publications, such as [38]. The relative speed-up by switching to multi-bank memory may approach $P$ for algorithms such as FFT [8]. 
Table 8.1: Comparison of parallel memory organizations.

\begin{tabular}{|c|c|c|c|c|}
\hline & \multicolumn{3}{|c|}{ Cycles per access } & \multirow[b]{2}{*}{ 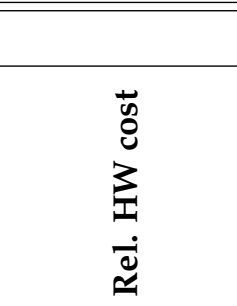 } \\
\hline Arch. & 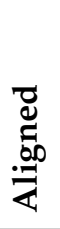 & 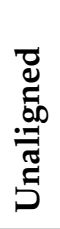 & 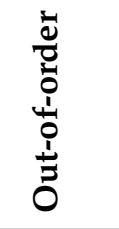 & \\
\hline Single-port single-bank & 1 & 2 & $\leq P$ & 1 \\
\hline Single-port multi-bank & 1 & 1 & 1 & $\sim C_{M B}$ \\
\hline Multi-port multi-bank & 1 & 1 & 1 & $\sim P_{\text {ports }} \cdot C_{M B}$ \\
\hline
\end{tabular}

\subsection{Multi-bank memories}

This section will discuss trade-offs regarding the design of a local memory subsystem based on multi-bank memory. Since many processor designs are dominated by memory area and memory energy consumption, these parameters will have a huge impact on processor characteristics.

\subsubsection{Terminology}

In order to discuss parallel memory access and multi-bank memory, an appropriate terminology will now be introduced. The terminology is not the same as, but largely inspired by [74], which also discusses the theory about parallel memory access in greater detail.

Consider a parallel memory with $B$ memory banks, as shown in Figure 8.2. Each bank is of size $M_{b}$ memory words and the total size of the overall memory is $M=B \cdot M_{b}$. For simplicity we assume that each bank may service maximally a single request per clock cycle. In addition to the memory modules, the parallel memory also includes address generation circuitry, which generates the addresses for data selection, duplication and the desired order of elements. The actual rearrangement of the data is handled by a permutation network.

A logical memory address $\alpha$ is translated to a corresponding phys- 


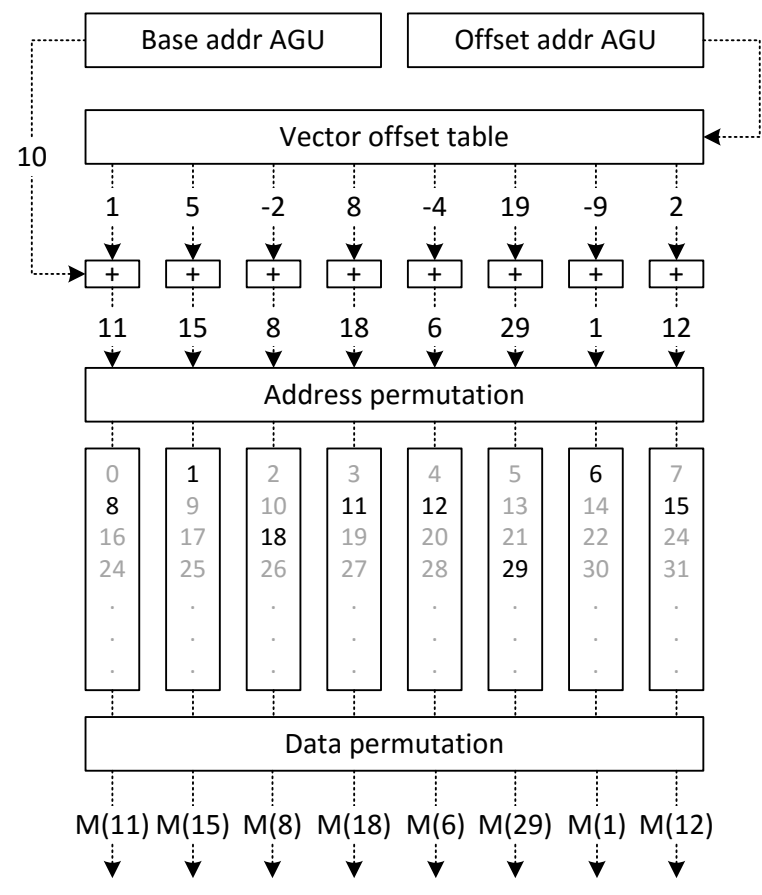

Figure 8.2: A local memory with address generation units (AGUs).

ical bank $b \in\{0, \ldots, B-1\}$ and physical address within that bank $\beta \in$ $\left\{0, \ldots, M_{b}-1\right\}$, using a bank assignment function $\Psi_{b}(\alpha)$ and address assignment function $\Psi_{\beta}(\alpha)$ respectively. To fully utilize the memory, each logical memory address must be translated into a unique $(b, \beta)$-pair.

In order to address a $B$-way parallel memory, $B$ logical memory addresses have to be generated. We subdivide this problem into the generation of a scalar base address $\alpha_{\text {base }}$ and a permutation vector $\alpha_{\pi}$. The logical vector address is typically generated as

$$
\alpha_{i}=\alpha_{\text {base }}+\alpha_{\pi, i} \quad \text { for } i \in\{0, \ldots, B-1\}
$$

although more complex formulations is certainly possible and sometimes beneficial [75]. The translation to a physical location may be performed 
with the following equations

$$
\begin{array}{ll}
b_{i}=\Psi_{b}\left(\alpha_{i}\right)=\alpha_{i} \bmod B & \text { for } \alpha_{i} \in\{0, \ldots, M-1\} \\
\beta_{i}=\Psi_{\beta}\left(\alpha_{i}\right)=\left\lfloor\alpha_{i} / B\right\rfloor & \text { for } \alpha_{i} \in\{0, \ldots, M-1\}
\end{array}
$$

These bank- and address assignment functions results in the logical address layout of Figure 8.2. These functions are not always the best choice, as we shall see later. However, they result in a very simple hardware implementation, especially when $B \in\left\{2^{x}: x \in \mathbb{N}\right\}$.

The possibly most interesting property of a parallel memory access is whether a certain memory access is conflict-free with respect to the bank assignment function or not.

Definition: A bank assignment function $\Psi_{b}$ is called conflict-free with respect to a vector address $\alpha$, if all pairs of addresses $\alpha_{i}$ and $\alpha_{j}$, when $\alpha_{i} \neq \alpha_{j}$ result in

$$
\Psi_{b}\left(\alpha_{i}\right) \neq \Psi_{b}\left(\alpha_{j}\right), \quad i, j \in\{0, \ldots, B-1\}
$$

This definition implies that a vector address $\alpha$ resulting in multiple requests to any given bank is not conflict-free w.r.t. the bank assignment function, if the requests ask for different physical addresses $\beta$ within the bank. If all requests are to the same physical address, the vector address is still conflict-free. This is necessary to support duplication without any performance loss.

\subsubsection{Memory bank granularity}

One of the most important design parameters of a multi-bank memory is the bank granularity: how many bits a memory word should consist of. In the extreme case, the width of a single bank is only a single bit, which means that the memory can support out-of-order access on the bit-level. Fine-grained multi-banking comes with great cost: narrow banks will lead to higher area cost per memory bit and higher energy consumption. Additionally, more memory addresses have to be generated, which complicates address generation. 
Table 8.2: Typical data width requirements for a few selected applications.

\begin{tabular}{l|c}
\hline \hline Application & Bit widths \\
\hline Communications & $16-32$ \\
Forward Error Correction (FEC) & $1 / \#$ softbits \\
Image processing & $8-16$ \\
Media & $(1) / 8-16$ \\
Radar & 32 \\
\hline \hline
\end{tabular}

The best choice of bank granularity is highly dependent on the application's needs. Table 8.2 summarizes some typical data type widths for a few selected workloads. It is however not enough to simply determine the required data types. In principle one only needs enough granularity to avoid bottlenecks for the most frequently executed unaligned- or outof-order accesses of the application, since straight-line aligned accesses can be handled easily with only a single bank. Furthermore, if some fine-grained out-of-order accesses are not performance critical, one may temporarily convert a smaller data type, or handle the fine-grained accesses with lower performance. Sometimes it may even be possible to do this without any performance degradation.

Table 8.3 shows an example of the relative cost of multi-banking. This only covers the cost of the actual memory modules, but this is typically dominating over all other hardware costs by far. This example uses a natural vector width of 128 bits and the results are gathered from 65nm CMOS memories. The cost is normalized to the 128 bits per bank case, which corresponds to single-bank memory. The actual relative cost depends on the size of the memory. Each row represents a specified number of 128-bit vectors. The general trend is that the relative cost per bit decreases as the memories gets larger, but the overhead is still large for larger fine-grained multi-banked memories. The choice of a fine-grained multi-banking setup has to be carefully motivated. 
Table 8.3: Relative cost of multi-banking for 128-bit wide memories in $65 \mathrm{~nm}$ CMOS as a function of memory size (the total number of 128-bit vectors). Each row has been normalized in relation to the single-banked case (128 bits per bank).

\begin{tabular}{l|cccccc}
\hline \hline & \multicolumn{7}{|c}{ Bits per bank } \\
\# vectors & 1 & 8 & 16 & 32 & 64 & 128 \\
\hline 256 & 11.3 & 2.13 & 1.60 & 1.24 & 1.07 & 1 \\
512 & 8.0 & 2.25 & 1.63 & 1.25 & 1.09 & 1 \\
1024 & 6.3 & 2.04 & 1.57 & 1.25 & 1.10 & 1 \\
2048 & 5.7 & 1.78 & 1.56 & 1.24 & 1.14 & 1 \\
4096 & 4.6 & 1.57 & 1.33 & 1.22 & 1.07 & 1 \\
\hline \hline
\end{tabular}

\subsubsection{Memory width}

So far the discussion has assumed that the natural processing width of $\nu$ bits per vector operand and clock cycle also means that each multibank memory has the same width. Consider, as an example, a parallel datapath that processes 128-bit per operand and cycle. This means that the architecture would be a 16-way architecture for 8-bit data types, 8way architecture for 16-bit data types and 4-way architecture for 32-bit data types. In such a case, the total width of a multi-bank vector memory would also be 128 bits wide, divided into $B$ memory banks, each of width $\nu_{b}=\nu / B$ bits.

\section{Use cases for $B>P$-memories}

Assume that each of the $P$ datapath lanes consume the same number of bits $\nu_{\text {lane }}$ as the width of a memory bank $\nu_{b}$. Further assume that the memory vector accesses request data from $P$ different conflict-free addresses. In order to avoid a memory bottleneck, the number of memory banks should be $B \geq P$. If $B>P, P$ memory banks will be activated and $B-P$ memory banks will remain inactive with each memory request. This may seem like a waste of memory banks, but there are several reasons why one may consider this approach. One reason would be to 
increase total memory size, although it may be more efficient to simply increase the size of the banks, rather than adding more banks. A better reason would be to avoid memory conflicts.

Increasing the number of banks beyond $P$ would leave no benefits for consecutive addressing. However, a very common access pattern is strided accesses, here called stride- $n$, where $n$ represents the distance between two elements. The SIMD parallelism $P$ of most processors is typically chosen as $P=2^{x}$ for some $x \in \mathbb{N}$. Unfortunately, the most common strides are also powers of two. If the number of banks $B$ is a power of two, we frequently will end up with non-conflict-free memory access using the standard bank assignment function of Equation 8.2.

As an example, consider the stride-2 vector access $\alpha=[0,2,4,6]$ for a processor with $P=B=4$. Addresses 0 and 4 would be mapped to memory bank 0 and addresses 2 and 6 would be mapped to memory bank 2. This access is therefore not conflict-free. However, if the number of banks $B$ is increased, especially to a value that is relatively prime in relation to $P$, any stride- $2^{x}$ access of $P$ elements would be conflict-free w.r.t. to the standard bank assignment function [74]. If $B=5$, we instead get $\Psi_{b}(\alpha)=[0,2,4,1]$ which clearly are all different memory banks. Using this memory, stride-5 accesses (and other multiples of 5) would not be conflict-free, but this stride is much less common in practice.

The positive aspects of using a prime number of memory banks was early identified and implemented in some supercomputer designs [76]. In addition to the extra memory bank(s), there will be an increased cost for logical- to physical address translation, since $\Psi_{b}$ and $\Psi_{\beta}$ will require fixed-divisor dividers and modulo hardware. It is possible to modify these functions into

$$
\begin{aligned}
& \Psi_{b}(\alpha)=\alpha \bmod B \\
& \Psi_{\beta}(\alpha)=\left\lfloor\alpha / M_{b}\right\rfloor
\end{aligned}
$$

and the whole memory will still be generated if $B$ is a prime number larger than 2 and the memory bank size $M_{b} \in\left\{2^{x}: x \in \mathbb{N}\right\}$, which at least simplifies the division [74]. 
There are two good reasons to not choose $B>P$, besides hardware cost. First of all, if $B$ and $P$ are chosen to be relatively prime to avoid bank conflicts, it becomes less obvious how to handle data types of different bit widths. All discussion so far has implicitly assumed that there is only a single data type width. In practice the processor may have to support multiple of the standard data type widths of 8, 16, 32 or 64 bits, or possibly some application-specific bit width(s). As an example, an 8-way 16-bit processor with 9 memory banks, would leave 4.5 memory banks per word for 32-bit data types. However, the strongest reason why $B>P$ may be unnecessary is because DSP kernels typically have statically known or predictable data access patterns, which means that data can be prepared (rearranged) to support conflict-free data access anyway. If vector addresses are guaranteed to be free from conflicts, we get predictable performance and can simplify the hardware design.

\section{Use cases for $B<P$-memories}

Consider an application whose execution time is completely dominated by kernels which perform overlapped memory accesses, meaning that $\alpha_{i}=\alpha_{j}$ for at least some pair $i \neq j, i, j=\{0, \ldots, P-1\}$. In such a case it may be beneficial to choose $B<P$ to save memory cost. Alternatively, if a HOF architecture has been equipped with $P_{d p}$ parallel $P$-way datapaths which perform overlapped accesses, we may choose $B<P_{d p} \cdot P$. If we want to guarantee predictable performance, we must guarantee that any vector access at most requests $B$ unique addresses.

One example which would be benefit from this setup is an architecture that has been designed for high-performance signal filtering, by implementing $P_{d p} P$-way datapaths that can perform multiply- and accumulate reduction. If we consider a general FIR-filter implementation, where each datapath processes a single output sample at a time, we can approach a relative speed-up of $P_{d p}$ using $P_{d p}$ datapaths (compared to a single one) with only $B=P+P_{d p}-1$ memory banks. In principle we get a relative speed-up of $P_{d p}$ with only $\sim\left(P+P_{d p}-1\right) / P$ times extra hardware cost, since memories typically dominate implementation cost. 


\subsubsection{Memory size}

A final important parameter of any memory is its size. There are no easy guidelines for its selection. However, kernels which read the same data multiple times benefit significantly if all data can be kept in the local memory. An FFT implementation which requires multiple passes over the data is a good example. If intermediate data has to be stored in offchip memory, it is not unlikely that the performance may decrease (and energy consumption increase) by at least an order of magnitude.

\subsection{Conflict-free parallel memory access}

This section will discuss how to achieve conflict-free access of multibank memory under static access pattern assumptions. Static in this case implies that the data access patterns are known at the latest before kernel run-time and are not generated/modified during kernel computing. We require that they are known before kernel run-time, so that data access patterns potentially can be prepared in parallel with previous kernel computing, but not so that it influences the run-time of the kernel. This is a less strict requirement than requiring exact knowledge of the addressing pattern at compile-time, although this is typically the case.

The concept of conflict-free access will be illustrated through the following example: Consider the access of any $4 \times 4$ block of pixels from an image with width $W$ and height $H$, as shown in Figure 8.3. We will assume that the processing width and number of banks are equal $(P=$ $B$ ) for simplicity and that the standard bank assignment function from Equation 8.2 is used. The value of $P$ also reflects how many pixels the data access path will have to retrieve from memory every clock cycle. The values of $P$ and $B$ are of extreme importance, since they influence which memory accesses are conflict-free or not.

Assume that we want to access the rows of the pixel block consecutively. Given a base address to the pixel in the upper left corner of the pixel block (the base pixel), the rest of the pixels (including the base pixel) 


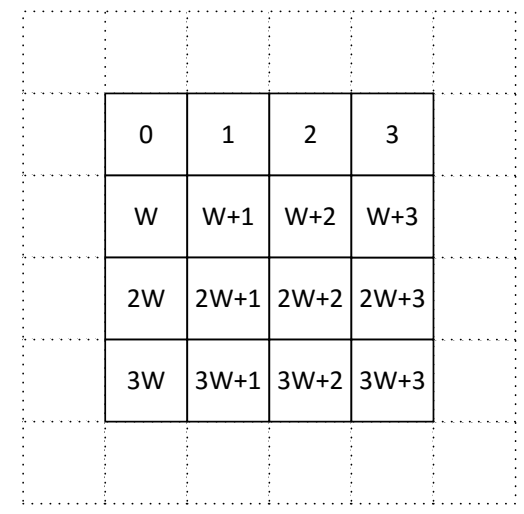

Figure 8.3: Accessing $4 \times 4$-blocks of pixels from an image. The values in the image represent the memory location offset of all pixels in the block relative the base pixel (pixel 0).

can be retrieved using the following vector of offsets:

$$
\begin{array}{r}
{[\phi(0,0), \phi(0,1), \phi(0,2), \phi(0,3), \phi(1,0), \phi(1,1), \phi(1,2), \phi(1,3),} \\
\phi(2,0), \phi(2,1), \phi(2,2), \phi(2,3), \phi(3,0), \phi(3,1), \phi(3,2), \phi(3,3)]
\end{array}
$$

where $\phi(a, b)=a \cdot W+b$. We call such a vector an offset vector and this vector may be used by a computing kernel to generate memory addresses using an address generator. If $W$ is a known fixed constant, the vector may be generated during compilation, otherwise it may be generated prior to kernel computing, as soon as the value of $W$ is known. When the standard bank assignment function is used, it is enough to know the offset vector and the given processing width $P$ to determine whether the generated memory accesses will be conflict-free or not.

Under a given processing width $P$, an offset vector of length $L$ will be subdivided into sub-vectors of length $P$ and the traversal of the offset vector will take at least $\lceil L / P\rceil$ cycles. An offset vector considered under a given processing width we refer to as a permutation table, which contains $\lceil L / P\rceil$ permutation vector entries. If $P$ does not evenly divide $L$, the last permutation table entry will be shorter than $P$. 
Using an architecture with $P=B=4$, the access of a $4 \times 4$-pixel block should take $\lceil 16 / 4\rceil=4$ clock cycles. The corresponding permutation table will be conflict-free, since each permutation vector corresponds to a single row of consecutive elements in the pixel block. However, under e.g. $P=B=8$, each permutation vector corresponds to the access of a rectangle of $2 \times 4$ pixels. This access may or may not be conflict-free, depending on the value of $W$, which has to satisfy $W=c \cdot B+4$, for an arbitrary constant $c \in \mathbb{N}$, to ensure that all pixels in the rectangle are stored in different memory banks. The issues with generating offset vectors that are conflict-free under multiple processing widths will be further discussed in Section 8.3.4.

To require that our input data structures have certain dimensions to ensure conflict-free access is certainly not an attractive solution and it is easy to find access patterns that will result in conflicts. Consider for example the case when we want to access the columns instead of the rows of the pixel block, corresponding to a stride- $W$ data access. If $W$ is a multiple of $B$ we are guaranteed to get conflicts for any $P>1$. We could of course store the image column by column instead to solve this issue. However, what happens if we need both row access and column access? This frequently occurs in image- and video compression standards such as JPEG and H.264 [77, 78]. To solve this issue, we need some other data distribution. We'll consider two different approaches in this work: permutation and padding.

\subsubsection{Permutation}

The usage of permutation simply implies that we rearrange the order of elements in memory to completely avoid, or at least minimize, the number of bank conflicts for the access patterns that we want to support. Consider that we want to support both row-wise and column-wise conflict-free access of the pixel blocks from Figure 8.3 under $P=B=4$. Further assume that $W=c \cdot B, c \in \mathbb{N}$, meaning that all pixels in any column end up in the same bank using row-wise storage. By simply rotating each row a different number of times, as shown in Figure 8.4, 


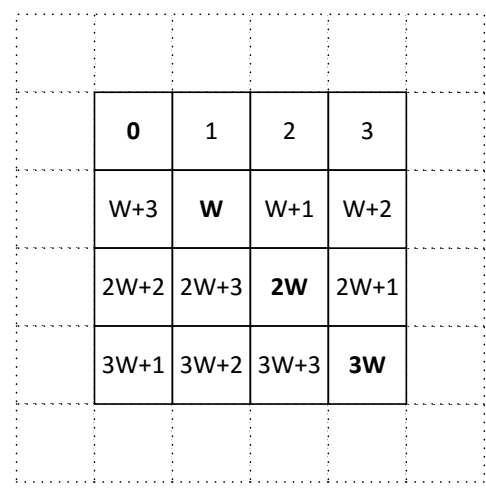

Figure 8.4: Conflict-free row- and column-wise access under $P=B=4$ enabled by permutation of rows.

both row- and column accesses can be made conflict-free. The following permutation vectors allow access to the rows and columns:

$$
\begin{aligned}
\alpha_{\pi}(\text { row } 0) & =[\phi(0,0), \phi(0,1), \phi(0,2), \phi(0,3)] \\
\alpha_{\pi}(\text { row } 1) & =[\phi(1,1), \phi(1,2), \phi(1,3), \phi(1,0)] \\
\alpha_{\pi}(\text { row } 2) & =[\phi(2,2), \phi(2,3), \phi(2,0), \phi(2,1)] \\
\alpha_{\pi}(\text { row } 3) & =[\phi(3,3), \phi(3,0), \phi(3,1), \phi(3,2)] \\
\alpha_{\pi}(\operatorname{col} 0) & =[\phi(0,0), \phi(1,1), \phi(2,2), \phi(3,3)] \\
\alpha_{\pi}(\operatorname{col} 1) & =[\phi(0,1), \phi(1,2), \phi(2,3), \phi(3,0)] \\
\alpha_{\pi}(\operatorname{col} 2) & =[\phi(0,2), \phi(1,3), \phi(2,0), \phi(3,1)] \\
\alpha_{\pi}(\operatorname{col} 3) & =[\phi(0,3), \phi(1,0), \phi(2,1), \phi(3,2)]
\end{aligned}
$$

One positive aspect of permutation is that no extra data memory is consumed, since elements are only rearranged. The main drawback is that we may require many permutation vectors, which will consume storage space. In addition, if we want to perform random access of rows and columns, it may take extra time to figure out which permutation vector to apply, once we know what row/column to access. The problem of finding conflict-free permutation tables can be formulated as e.g. an ILPproblem (Integer Linear Programming) and be solved automatically [46]. 


\subsubsection{Padding}

An alternative to permutation is to use padding. By inserting dummy elements at well-chosen locations (in this case after each image row) we can avoid bank conflicts, as shown in Figure 8.5. Any row or column may then be accessed with the same row/column permutation vector, by simply supplying the address to where the row or column starts. The row and column permutation vectors are

$$
\begin{aligned}
\alpha_{\pi}(\text { row }) & =[\phi(0,0), \phi(0,1), \phi(0,2), \phi(0,3)] \\
\alpha_{\pi}(\operatorname{col}) & =[\phi(0,0), \phi(1,1), \phi(2,2), \phi(3,3)]
\end{aligned}
$$

and the actual addresses of the rows/columns can be calculated using the relation $\alpha=\alpha_{\text {base }}+\alpha_{\pi}$, which results in

$$
\begin{array}{rlrl}
\alpha(\text { row }) & =0(W+1)+\alpha_{\pi}(\text { row }) & =[\phi(0,0), \phi(0,1), \phi(0,2), \phi(0,3)] \\
\alpha(\text { row } 1) & =1(W+1)+\alpha_{\pi}(\text { row }) & =[\phi(1,1), \phi(1,2), \phi(1,3), \phi(1,4)] \\
\alpha(\text { row } 2) & =2(W+1)+\alpha_{\pi}(\text { row }) & =[\phi(2,2), \phi(2,3), \phi(2,4), \phi(2,5)] \\
\alpha(\text { row } 3) & =3(W+1)+\alpha_{\pi}(\text { row }) & =[\phi(3,3), \phi(3,4), \phi(3,5), \phi(3,6)] \\
\alpha(\text { col } 0) & =0+\alpha_{\pi}(\text { col }) & & =[\phi(0,0), \phi(1,1), \phi(2,2), \phi(3,3)] \\
\alpha(\text { col } 1) & =1+\alpha_{\pi}(\text { col }) & & =[\phi(0,1), \phi(1,2), \phi(2,3), \phi(3,4)] \\
\alpha(\operatorname{col} 2) & =2+\alpha_{\pi}(\text { col }) & & =[\phi(0,3), \phi(1,4), \phi(2,5), \phi(3,6)] \\
\alpha(\text { col } 3) & =3+\alpha_{\pi}(\text { col }) &
\end{array}
$$

Compared to permutation we require fewer permutation vectors and can reuse them for any row/column access. The downside is that the data structure requires more memory space ${ }^{1}$. As with permutation, suitable padding layouts may be found through ILP [47].

\footnotetext{
${ }^{1}$ It can be argued whether or not the padding elements actually waste memory space, since the padding space can be filled with other unrelated, yet still useful data. Consecutive addressing of padding elements will often even be free from memory conflicts.
} 


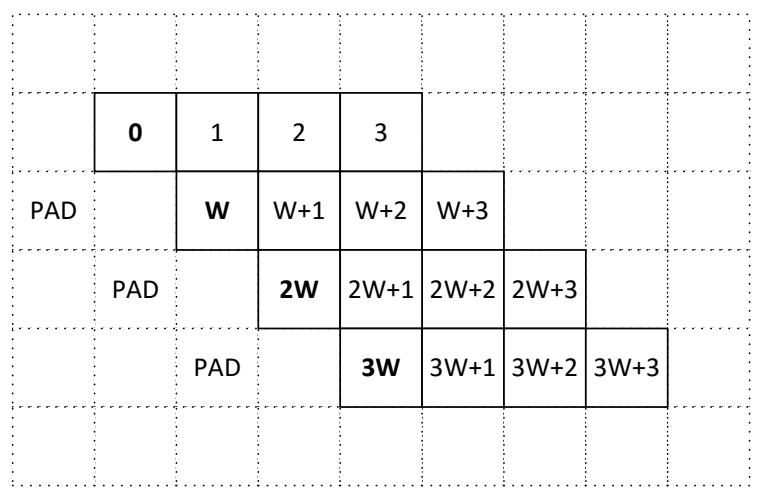

Figure 8.5: Conflict-free row- and column-wise access under $P=B=4$ using padding.

\subsubsection{Multiple buffering}

A very useful technique to speed-up processing is to use multiple buffering. When a complete data structure is going to be updated, we might instead create a new buffer at a new location and store the result there. If we have access to multiple data memories, we may even store the data structure in a different memory, to avoid memory resource conflicts during processing, without having to use multi-port memory. The new buffer doesn't necessarily have to use the same storage layout as the old one, and may instead be modified to allow conflict-free memory accesses for subsequent accesses to the buffer. If we fill an entire buffer once and then read each element of the buffer maximally once (write-once-readonce), it is always possible to find a permutation table that is conflict-free, regardless of the read- and write access patterns [79].

To illustrate the use of conflict-free memory access under multiple buffering, we shall consider the implementation of two-dimensional Discrete Cosine Transform (2D-DCT) and quantization, which is a part of image/video-compression standards that typically require both row- and column access to pixels in a pixel block. Regardless of the exact 2D-DCT variant used, we can typically sub-divide the computation into two 1DDCTs, by first applying 1D-DCT to all rows of the block, followed by 1D-DCT on the columns. The quantization step is a simple element-wise 
Listing 8.1: Pseudocode for 2D-DCT.

$1 \mathrm{~A}=\operatorname{transpose}(1 \mathrm{~d}-\operatorname{dct}(\mathrm{A}))$

$2 A=\operatorname{transpose}(1 d-\operatorname{dct}(A))$

$3 \mathrm{~A}=$ elementwise-multiply (Quant, A)

multiplication of the 2D-DCT result, with a fixed quantization matrix. This can be implemented, as shown in Listing 8.1, where $A$ is an $n \times n$ pixel block, Quant is an $n \times n$ quantization matrix and the function $1 d-d c t$ performs row-wise 1D-DCT to all rows of the input matrix/block. Given that we have implemented flexible multi-bank memory access with permutation, we can eliminate the execution time overhead of transpose that is unavoidable on most other architectures.

Once again, assume that $P=B=4, W=c \cdot B, c \in \mathbb{N}$, the pixel block size is $4 \times 4$ pixels and that the image is originally stored row-wise. The first 1D-DCT can easily be applied to all rows, since the elements of the rows are stored consecutively. However, if the result of the first 1D-DCT is written back to the original location in the natural order, it will be impossible to read the columns conflict-free for the column-wise 1D-DCT. If we however write the result to a new location, and at write-time apply some technique such as permutation or padding, we can make sure that the subsequent column accesses are conflict-free. The quantization step can finally restore the original data layout if desired. The procedure is shown in Figure 8.6. This example clearly demonstrates the advantages of having both read- and write permutation capability.

The proposed method has several advantages. First of all, if data access is orthogonal to computing, it is possible to remove any cycle time overhead of the data access pattern in this example. Furthermore, the use of multiple buffers allows us to hide the addressing complexity inside the kernel, since the compute kernel can use simple row-wise storage for input and output. Finally, since data access is not a bottleneck (it can deliver $P$ elements per clock during the whole computing process), we can use an arbitrarily complex $P$-way datapath, to reach a datapath 


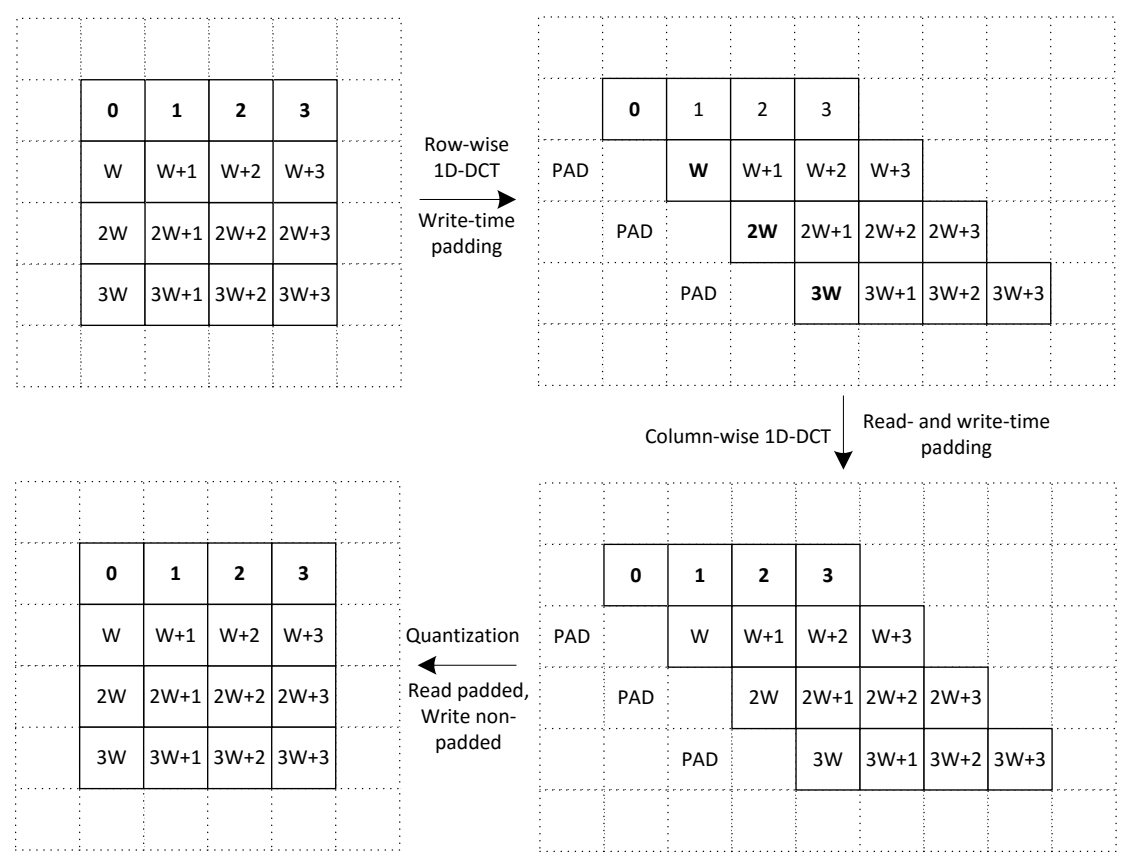

Figure 8.6: Conflict-free memory access under multiple buffering.

throughput of one 1D-DCT per clock cycle, resulting in high processing speed.

\subsubsection{Scalability}

The HOF instruction concept is naturally scalable to any processing width $P$. The same goes for the memory subsystem and address generation circuitry, which at least theoretically can be scaled to support any number of banks or an arbitrary bank granularity. However, great care has to be taken regarding permutation tables, which we demonstrate in the following example.

Consider the problem of transposing a $4 \times 4$ matrix. To perform this operation with one read-write operation per matrix element, one may read the matrix data as shown in Figure 8.7(a). Considering a processor with $P=B=4$ and the standard bank assignment function from Equa- 


\begin{tabular}{|c|c|c|c|c|c|c|}
\hline 0 & 1 & 2 & 3 & 0 & 5 & 10 \\
\hline 4 & 5 & 6 & 7 & 8 & 13 & 2 \\
\hline 8 & 9 & 10 & 11 & 4 & 9 & 14 \\
\hline 12 & 13 & 14 & 15 & 12 & 1 & 6 \\
\hline
\end{tabular}

$\begin{array}{ll}\text { (a) A } 4 \times 4 \text { matrix. } & \text { (b) A possible conflict-free permuta- }\end{array}$ tion table for $P=B=4$.

\begin{tabular}{llllllll}
\hline $\mathbf{0}$ & $\underline{5}$ & 10 & 15 & $\mathbf{8}$ & $\underline{13}$ & $\mathbf{2}$ & 7 \\
4 & $\underline{9}$ & 14 & 3 & 12 & $\underline{1}$ & 6 & 11
\end{tabular}

(c) The same table under $P=B=8$ has bank conflicts.

$\begin{array}{rrrrrrrr}0 & 5 & 10 & 15 & 4 & 9 & 14 & 3 \\ 8 & 13 & 2 & 7 & 12 & 1 & 6 & 11\end{array}$

(d) A rearranged table which is conflict-free for $P=B=$ $2^{n}, n \in \mathbb{N}$.

Figure 8.7: A practical example of the potential problems with permutation tables when scaling processing width.

tion 8.2, one may use the permutation table depicted in Figure 8.7(b). However, if the system is later scaled to $P=B=8$, the resulting permutation table is not conflict-free, as shown in Figure 8.7(c). The permutation table would have been conflict-free, if the 4-element vectors instead were processed in the order given in Figure 8.7(d). On the other hand, note that both permutation tables are conflict-free for $P=B=16$. This raises the question whether a particular kernel binary can be compatible 
with any architecture.

Scalability can be considered as an additional constraint when generating permutation tables. In general however, it is impossible to support scalability to any processing width and still maintain maximum possible performance. For example, a $P$-way architecture will require permutation tables of size $c \cdot P, c \in \mathbb{N}$, to maximize processor utilization and therefore there is no upper bound on the required permutation table size. A better practical solution is possibly to formulate the data access pattern in a processing width independent way, and then generate/compile code for the current width, since the generation of a permutation table for a given processing width can be performed automatically [8]. Alternatively, one can generate tables that are conflict-free for a smaller set of practically useful processing widths, such as a few powers of 2 .

\subsection{Kernels and memory parallelism}

The two previous sections primarily discussed the access of a single $P$ way operand stored in some (multi-banked) data memory. However, as previously mentioned, an instruction may require up to $3 P$ memory operations per cycle, in case the instruction is a binary map or block-map instruction. This section will discuss the practical need of different kernels in order to find possible opportunities for hardware reduction without significantly sacrificing performance.

Chapter 3 classified some typical DSP kernels into categories, a categorization that now will be repeated and used to discuss memory bandwidth requirements. Although there is some overlap between categories, kernels can be roughly categorized as:

- Simple element-wise: This category includes kernel that just perform simple unary and binary arithmetic and logical operations such as addition, subtraction, absolute value, minimum, maximum, and, or, xor and more. These operations can be described by the HOF map pattern, requiring $2 P$ (for unary) or $3 P$ (for binary) data access operations per cycle. However, quite frequently the require- 
ments are reduced, because one input operand is a scalar constant or a vector of constants.

- Reductions: Operations such as calculating the sum of a vector, or minimum/maximum of each row/column of a matrix are included in this category. These operations map to reduce and block-reduce patterns.

- Filters: Filter-kernels typically perform binary block-map-reduce operations, where one of the two input operands is a fixed set of constants, so called filter coefficients.

- Transforms: Transforms such as FFT and DCT perform binary blockmap, with one operand being constant transform coefficients (in case of FFT called twiddle factors).

- Math functions: Math functions, such as transcendental operations, are similar to the simple element-wise operations, but are significantly more complex to implement computation-wise. If implemented by software, intermediate results may be stored in a register file to reduce memory requirements. If implemented in hardware they belong to the map pattern, but may require much less memory bandwidth than usual, because each individual operation can require many cycles to complete. These operations are typically computed using some iterative approach, series expansions or lookup tables.

- Vector operations: Vector operations in this context are operations which perform some transformation of a complete vector, where the operation cannot be seen as operations on individual vector elements. Examples include sorting the vector, prefix operations and interleaving. These kernels typically require several different instructions to complete, but they are often of the unary block-map type.

- Matrix operations: Examples of matrix operations include matrix multiplication, transpose, inversion, and decompositions such as 
Table 8.4: A summary of typical data access bandwidth requirements for different kernel categories. In addition, the use of constant data (such as filter and transform coefficients) is indicated.

\begin{tabular}{l|cc}
\hline \hline Category & Constants? & Data access ops./cyc \\
\hline Simple element-wise & Yes/No & $2 P / 3 P$ \\
Reductions & No & $P(+1) /(2 P(+1))$ \\
Filters & Yes & $2 P(+1)$ \\
Transforms & Yes & $3 P$ \\
Math functions & Yes/No & $<2 P$ \\
Vector operations & No & $2 P / 3 P$ \\
Matrix operations & No & $3 P$ \\
Table access & No & $2 P$ \\
\hline \hline
\end{tabular}

Cholesky, QRD, LUD and SVD. While some are simple data rearrangements, other operations require a complex implementation with many instructions of the binary map type with few opportunities for simplification.

- Table access: Kernels using table access (e.g. table lookup or histogram generation) typically perform very simple computations, but perform data dependent memory access, which goes against the data access predictability of most other kernels. Unpredictable data access will be further discussed in Section 8.6.

Table 8.4 summarizes typical data access bandwidth requirements of these kernel categories, presented as number of data access operations required per cycle. It also lists whether the category use constant data for its implementation, which creates some opportunities for hardware simplification. The following subsections will discuss such opportunities in greater detail. 


\subsubsection{Kernels with constant data}

Filters, transforms and to some extent simple element-wise operations and math functions, frequently use constant data for their implementation. Based on the kernels implemented in this work, the following observations have been made: Sometimes the set of constants is small and frequently reused, which is an excellent opportunity for register file storage. Also, the need for out-of-order and unaligned vector access to constants is rare, which makes it possible to use a single-banked memory configuration for constants. Of particular interest in this case is the instruction memory. Constant data may often be placed as part of the kernel binary and when a many-cycle HOF instruction is executing, the instruction memory would then be free to load constants, which would reduce the need of one data memory. This issue will be discussed in Chapter 9.

\subsubsection{Bandwidth requirements for reduction patterns}

Reduction and filter kernels use reduce, map-reduce, block-reduce and blockmap-reduce patterns with a worst-case memory bandwidth requirement of $2 P+1$ data elements per cycle. The actual memory pressure will depend on the number of elements in each reduction. For block patterns, an output element will be produced every $\left\lceil N_{i} / P\right\rceil$ cycles, where $N_{i}$ is the inner compute dimension (or number of elements per block).

To evaluate the practical performance one can expect from these patterns on different data access configurations, we shall now consider the performance of block-reduce or block-map-reduce on single, dual and triple multi-bank memory configurations. Each memory can either load an input vector or store the scalar output of a reduction when needed. Since output store operations do not use the entire width of a $P$-way memory, we also consider two configurations that include store buffers. These store buffers are used to buffer $P$ data elements before the output is actually written to memory. This analysis assumes that the target addresses of a buffered vector store operation does not result in bank conflicts.

The achieved performance is heavily dependent on the values of $P$ 


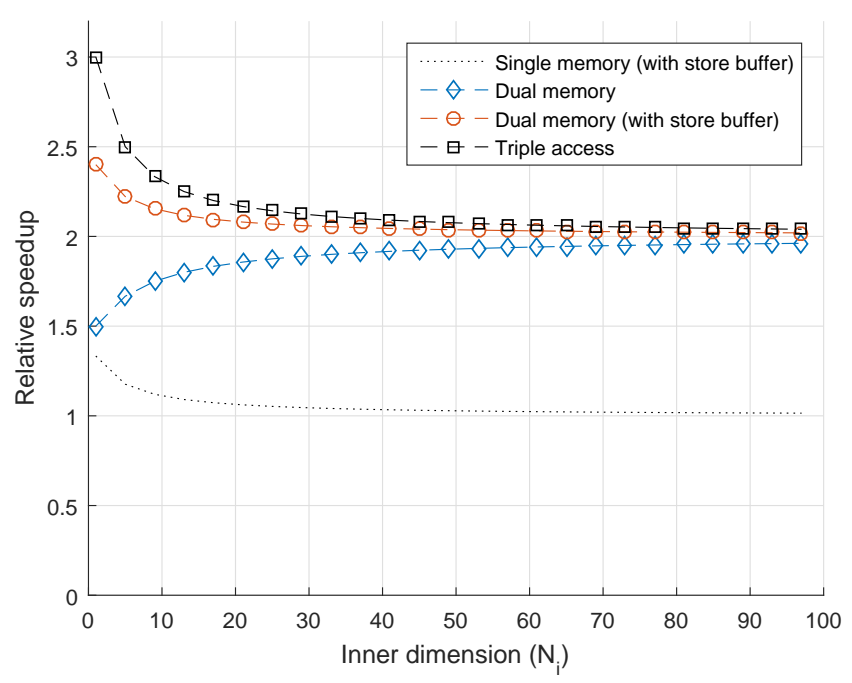

(a) Processing width $P=4$

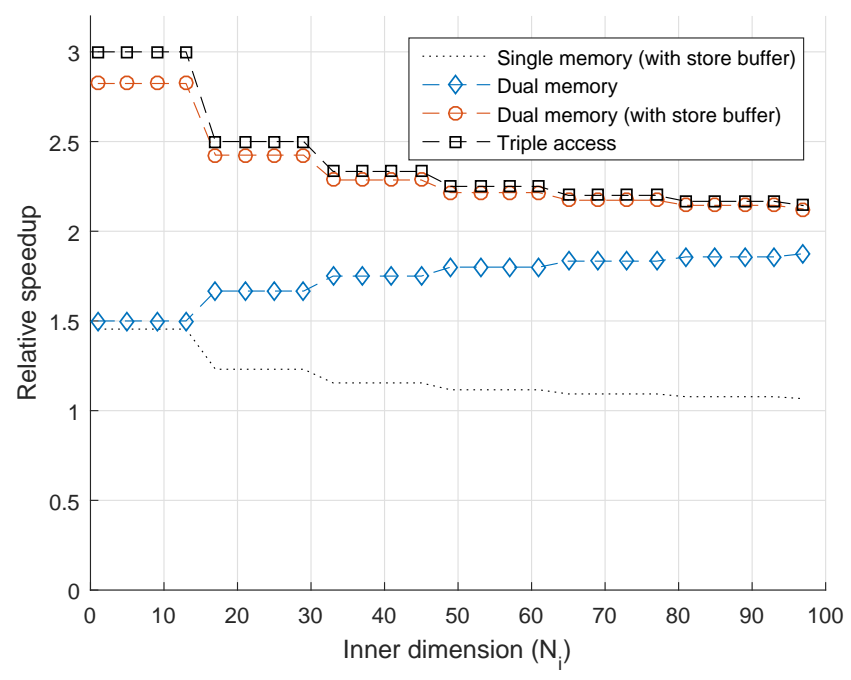

(b) Processing width $P=16$

Figure 8.8: Relative speedup of different memory configurations, compared to a single multi-bank memory without a store buffer. 
and $N_{i}$. Figures 8.8(a) and 8.8(b) show the relative speedup compared to a non-buffering single memory setup for $P=4$ and $P=16$ respectively. For small values of $N_{i}$ there are larger variations in performance and buffering has a significant effect. For larger $N_{i}$, output values are less frequently produced and buffering has less of an impact. The general conclusion is that more than two inputs memories does not have significant effects on performance, if buffering is introduced. Furthermore, if any operand is placed in e.g. a register file, there will be even less pressure on the memories.

\subsubsection{Bandwidth requirements for map patterns}

As we've seen earlier, kernels performing binary map and block-map require the most memory bandwidth. Transform kernels may potentially be simplified due to their use of constants. This primarily leaves matrix operation kernels as one of the most problematic.

The implementation of matrix operations often requires row / columnwise vector operations such as

$$
a=a-b \cdot c
$$

which can be used to implement QR- and LU-decompositions [80]. Since we do not consider ternary operations in this work, the operation must be subdivided into temp $=b \cdot c$ followed by $a=a-t e m p$. The vector temp may be placed in a register file to reduce the memory bandwidth requirements from $3 P$ to $2 P$ memory operations per cycle. The dual use of vector $a$ in the second operation can be resolved using multiple buffering. A potential problem is the length of vector temp, which may not always fit the register file and the operation may then have to be subdivided further. The general conclusion is that dual memory may sometimes be enough even for matrix operations, although considerably more effort may have to be placed on the kernel implementation and/or other hardware features. This will be further discussed in Section 11.8. 


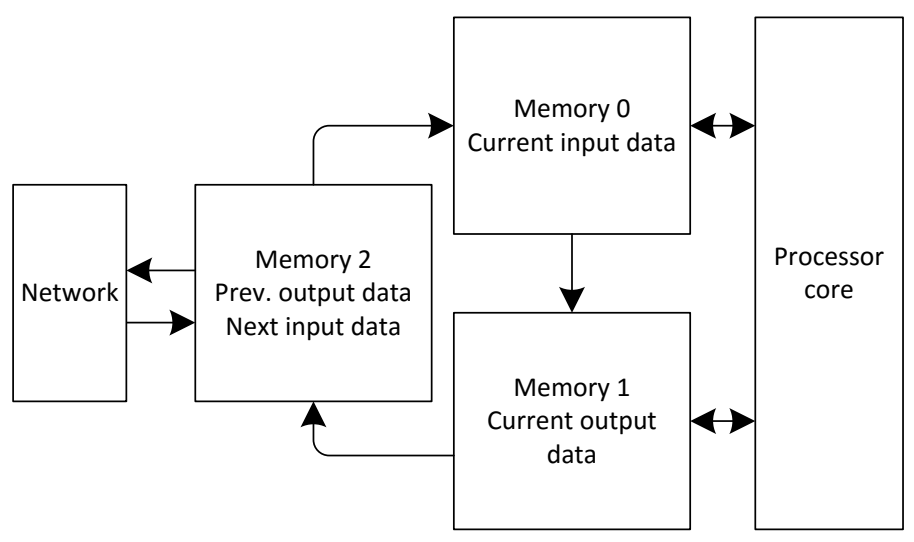

Figure 8.9: Overlapping main memory loads/stores with computing.

\subsubsection{System-level considerations}

The memory bandwidth requirements have so far been discussed in an isolated environment. In practice, loads and stores between off-chip main memory and local memories will consume additional local memory bandwidth. One solution that allows asynchronous loads/stores and still results in predictable kernel execution time is shown in Figure 8.9. By introducing a spare memory which is used for main memory transfers, data can be moved without interfering with kernel execution. When kernel execution has terminated, new input data may already be available in the spare memory. By a simple memory switch, the new input data can be handed over to the compute processor immediately and the newly generated output data may be transferred back to main memory.

The proposed solution can almost eliminate all inactive time for a compute core, but it is certainly not for free. Additionally, it requires a certain level of predictability of the overall software running on the system to guarantee that computing and data access can overlap and the main memory bandwidth must be sufficient. If the number of cores is increased without a corresponding increase in main memory bandwidth, the compute-to-bandwidth ratio of the executed kernels must eventually 
increase in order to avoid a main memory bottleneck. If the compute-tobandwidth ratio increases, the effect of overlapped computing and data access becomes smaller.

A potential compromise in this situation is to design the system with three local data memories per processor core. It allows the use of overlapped computing/data access if kernel computing is only marginally improved (or not improved at all) by increasing the number of memories of computing memories from two to three. At the same time, kernels that benefit from using three memories can still do so when desired.

\subsection{Address generation}

To achieve orthogonal computing and data access in practice, it is essential that memory address generation can be separated from datapath computing. In such a case, the features of the address generation units (AGUs) will determine what memory access patterns can actually be achieved. This will be the topic of this section.

An access pattern which is statically fully known can theoretically be fully tabulated and placed in a lookup table (LUT). If the LUT can be accessed in parallel with the access of the data memory, all run-time cycle cost of addressing can be eliminated, at least if the loading of the LUT from main memory to some local storage is ignored. This however results in very large LUTs and no potential for any run-time data access decisions.

In practice, most data access patterns can at least to some extent be compute-time-generated by an AGU with relatively simple arithmetic operations. Additions are very frequently used. The need for multiplications can often be eliminated through strength reduction and recursive address generation [81]. Memory addressing can be broken down into the following three subcomponents:

- Configuration-component: An AGU is pre-configured for memory addressing before use. This can include simple operations such as setting an address register, but more complex alternatives are cer- 
tainly possible. Since configuration is performed before the actual memory data is accessed, it may cost additional processor cycles, an overhead that we would like to minimize.

- Instruction-component: Information delivered by the instruction that wants to access the memory data. Typical information delivered by an instruction is how to use and update an AGU configuration and this is possibly the most critical addressing subcomponent. If the flexibility is too low, we may require frequent AGU reconfigurations that cost clock cycles. However, unnecessary flexibility costs in terms of a larger instruction word size.

- LUT-component: A LUT that is used together with the AGU configuration to generate the final memory addresses. In practice, we would like to minimize the use of the LUT component, as it costs in terms of memory storage, but at the same time this component is one important key to high performance data access.

To illustrate how the three components can typically be used, discuss different implementation trade-offs and start drafting the requirements on an AGU, we shall consider the implementation of an image filter.

\subsubsection{Specification by example}

Consider an application where image filtering using two-dimensional convolution kernels has been identified as one of the most critical processing tasks. All practically relevant convolution kernels for the application are separable, meaning that the 2D-kernel can be broken down into 1D row-wise filtering, followed by 1D column-wise filtering or vice versa [40]. To accelerate the computation, a $P$-way datapath, with $P \in$ $\left\{2^{x}: x \in \mathbb{N}\right\}$, has been designed so that it can accept a complete row of the image as a block to a block-map operation, $P$ pixels at a time. After some pipeline latency, $P$ pixels are generated per clock cycle. The datapath retains some internal state during processing, to handle the data dependencies between neighboring pixels. The radius of the filter kernel 


\begin{tabular}{|c|c|c|c|c|c|c|c|c|}
\hline $\mathbf{R}_{\mathbf{0}}$ & $\mathrm{G}_{0}$ & $\mathrm{~B}_{0}$ & $\mathbf{R}_{\mathbf{1}}$ & $\mathrm{G}_{1}$ & $\mathrm{~B}_{1}$ & $\mathbf{R}_{\mathbf{2}}$ & $\mathrm{G}_{2}$ & $\cdots$ \\
\hline PAD & $\mathbf{R}_{\mathbf{W}}$ & $\mathrm{G}_{\mathrm{W}}$ & $\mathrm{B}_{\mathrm{W}}$ & $\mathbf{R}_{\mathrm{W}+\mathbf{1}}$ & $\mathrm{G}_{\mathrm{W}+1}$ & $\mathrm{~B}_{\mathrm{W}+1}$ & $\mathbf{R}_{\mathrm{W}+\mathbf{2}}$ & $\cdots$ \\
\hline $\mathrm{B}_{2 \mathrm{~W}-1}$ & PAD & $\mathbf{R}_{\mathbf{2 W}}$ & $\mathrm{G}_{2 \mathrm{~W}}$ & $\mathrm{~B}_{2 \mathrm{~W}}$ & $\mathbf{R}_{\mathbf{2 W + 1}}$ & $\mathrm{G}_{2 \mathrm{~W}+1}$ & $\mathrm{~B}_{2 \mathrm{~W}+1}$ & $\cdots$ \\
\hline $\mathrm{G}_{3 \mathrm{~W}-1}$ & $\mathrm{~B}_{3 \mathrm{~W}-1}$ & PAD & $\mathbf{R}_{\mathbf{3 W}}$ & $\mathrm{G}_{3 \mathrm{~W}}$ & $\mathrm{~B}_{3 \mathrm{~W}}$ & $\mathbf{R}_{\mathbf{3 W + 1}}$ & $\mathrm{G}_{3 \mathrm{~W}+1}$ & $\cdots$ \\
\hline
\end{tabular}

Figure 8.10: Storage of an RGB image, with padded rows to allow conflict-free column access.

is not important in this example, as it only affects the internal structure and latency of the datapath.

A color image with dimensions $W=W I D T H$ and $H=H E I G H T$ has been placed in local memory. Each pixel is stored as a triplet of three color components, one component per memory bank and $B=P$. The exact image format is less important (typical examples could be RGB or $\mathrm{YCbCr}$ ), but since filters are often applied to each component separately, we need stride- 3 memory access to read a row of any component. Each row has already been padded with a dummy element, to allow later conflict-free column access. The storage layout is shown in Figure 8.10.

A simple non-vectorized pseudocode implementation is shown in Listing 8.2. The implementation of the actual filtering is hidden in the filterrow function, but in this context we are only interested in the data access patterns. Based on this pseudocode, it would be simple to generate the addresses required to access multiple values from the input image, although multiplication is required. A strength-reduced version of the same code is shown in Listing 8.3. The multiplications are removed by using recursive address generation. A recursive implementation may certainly be beneficial for non-vectorized code, but a direct hardware implementation is less attractive.

As discussed earlier, memory addresses can be generated using a scalar base address and a vector of memory offsets. Given the base address $\alpha_{\text {base }}$ of an image row, the entire row can be fetched using the offset 
Listing 8.2: A simple filter implementation, exposing the data access patterns.

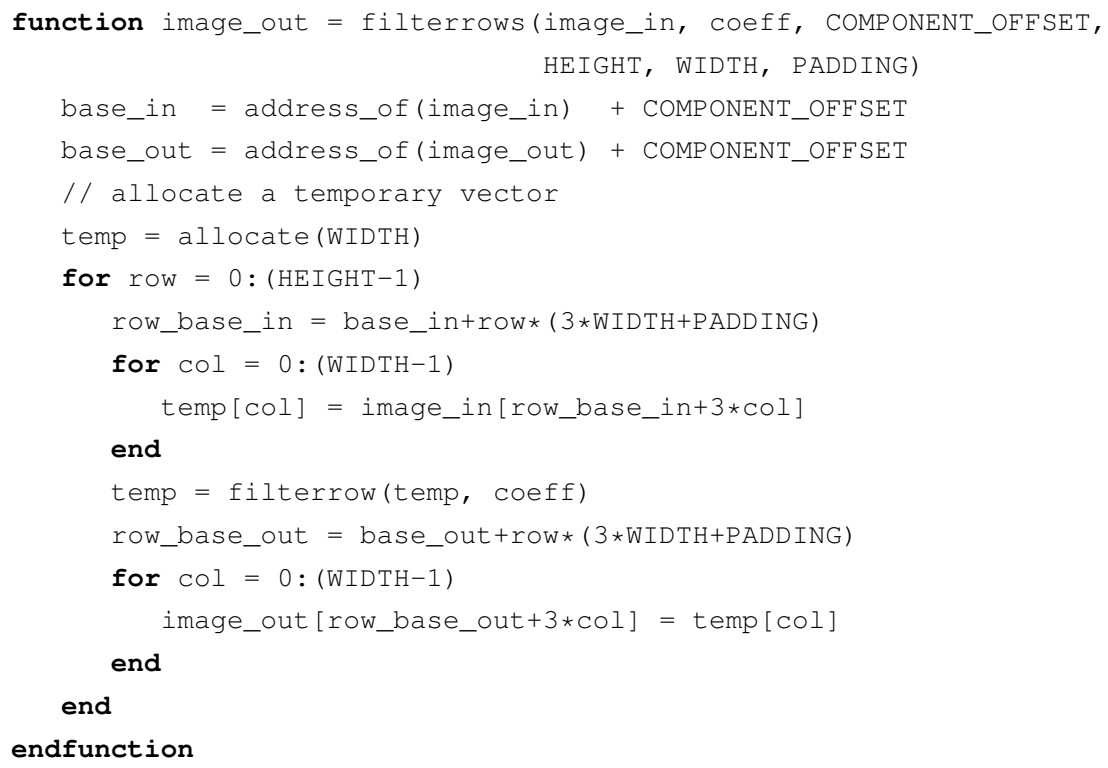

vector

$$
[0,3,6,9, \ldots, 3 \cdot(\text { WIDTH }-1)]
$$

resulting in an offset vector with WIDTH entries. Depending on processing width, $P$ entries from the offset vector would be used every clock cycle and logical memory addresses are calculated as

$$
\alpha_{i}=\alpha_{\text {base }}+\alpha_{\pi, i} \quad \text { for } i \in\{0, \ldots, P-1\} .
$$

The recursive address generation is then replaced with LUT access. The LUT solution has the advantage that it results in a simpler hardware implementation and supports more complex access patterns.

So far we have assumed that the scalar base address $\alpha_{\text {base }}$ remains constant during processing of an image row, and the offset vector is used to address all elements of interest, relative to the base address. However, this does not necessarily have to be the case and we can reduce permutation table size significantly if we allow $\alpha_{\text {base }}$ to change every clock cycle. 
Listing 8.3: The filter implementation, after applying strength reduction.

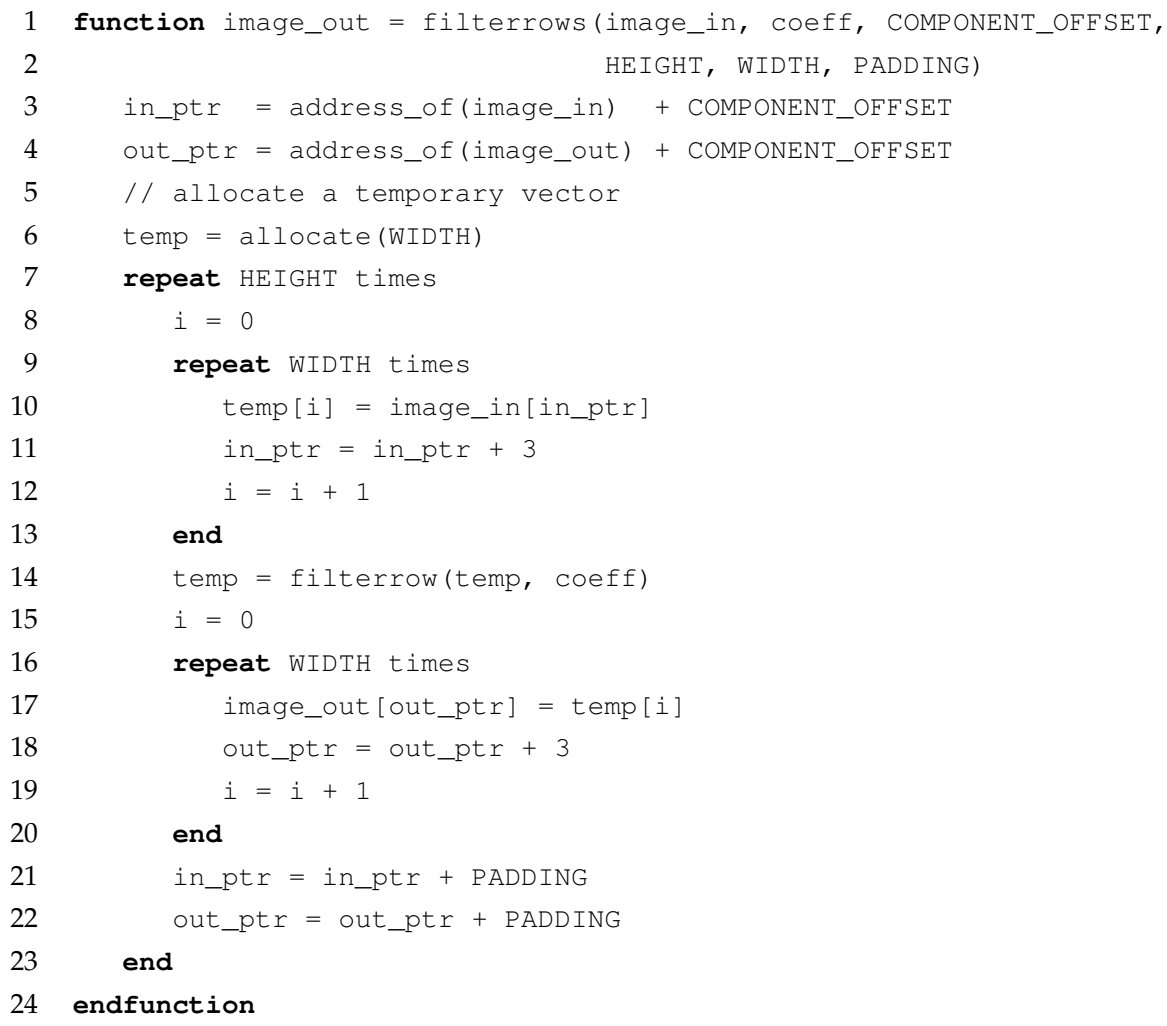


Consider two vector addresses that should be generated in two consecutive clock cycles, $\alpha^{i}$ and $\alpha^{i+1}$. Both are generated from the same base address $\left(\alpha_{\text {base }}^{i}=\alpha_{\text {base }}^{i+1}\right)$, but with different permutation vectors $\alpha_{\pi}{ }^{i}$ and $\alpha_{\pi}{ }^{i+1}$. All vectors are of length $P$. Let $\left|\alpha_{\pi}\right|$ denote the normalization of a permutation vector, where normalization is defined as

$$
\begin{aligned}
\left|\alpha_{\pi}\right| & =\alpha_{\pi}-\alpha_{\pi, 0} \\
& =\left[\alpha_{\pi, 0}-\alpha_{\pi, 0}, \alpha_{\pi, 1}-\alpha_{\pi, 0}, \ldots, \alpha_{\pi, P-1}-\alpha_{\pi, 0}\right] \\
& =\left[0, \alpha_{\pi, 1}-\alpha_{\pi, 0}, \ldots, \alpha_{\pi, P-1}-\alpha_{\pi, 0}\right]
\end{aligned}
$$

If $\left|\alpha_{\pi}{ }^{i}\right|=\left|\alpha_{\pi}{ }^{i+1}\right|$ (element-wise equality), we can generate the two vector addresses as

$$
\begin{aligned}
\alpha_{i}^{i} & =\alpha_{\text {base }}^{i}+\alpha_{\pi, i}^{i} \quad \text { for } i \in\{0, \ldots, P-1\} . \\
\alpha_{\text {base }}^{i+1} & =\alpha_{\text {base }}^{i}+\alpha_{\pi, 0}^{i+1}-\alpha_{\pi, 0}^{i} \\
\alpha_{i}^{i+1} & =\alpha_{\text {base }}^{i+1}+\alpha_{\pi, i}^{i} \quad \text { for } i \in\{0, \ldots, P-1\} .
\end{aligned}
$$

This means that we only need the first permutation vector, if $\alpha_{\text {base }}^{i+1}$ can be recursively generated from $\alpha_{b a s e}^{i}$. For the image processing example, the normalized permutation for all accesses is

$$
\left|\alpha_{\pi}\right|=[0,3,6, \ldots, 3 \cdot(P-1)]
$$

and if we only store $\left|\alpha_{\pi}\right|$ in memory, we can generate all addresses within an image row if $\alpha_{\text {base }}^{0}$ is properly initialized, and new base addresses are generated as

$$
\alpha_{\text {base }}^{i+1}=\alpha_{\text {base }}^{i}+3 P \quad \text { for } i \in\{0, \ldots,\lceil W I D T H / P\rceil-1\}
$$

By compressing the permutation vectors as proposed, we introduce a certain dependence to the processing width $P$. Whether this matters or not depends on the situation. For binary compatibility over multiple $P$, one must provide a permutation vector for the largest possible $P$. If code can be generated from source, one can generate code for any given $P$. Regardless of this, the flexibility is still higher than architectures providing 
Listing 8.4: Final pseudo-HOF-assembly implementation.

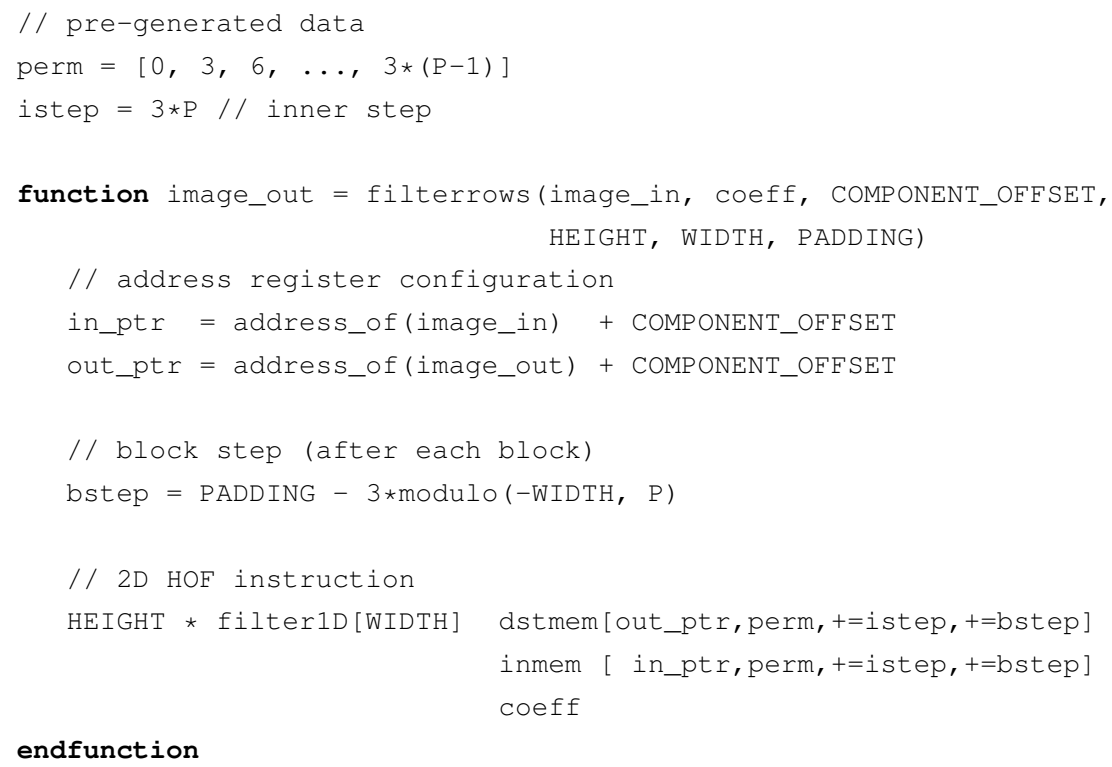

different instruction subsets/extensions for different processing widths, where not having a certain instruction means that some code cannot be executed at all.

To fully utilize the two-dimensional processing of HOF instructions, it must be possible to modify address pointers after each block has been processed. In this particular example, this may be used to compensate for the row padding. A final pseudo-assembly implementation is shown in Listing 8.4. The permutation vector and inner step applied to modify the $\alpha_{\text {base }}$ every clock cycle are pre-generated and delivered with the kernel binary. The input and output pointers are set and then continuously incremented during HOF processing. The block step, applied after each block, is calculated and compensates for the case where WIDTH is not a multiple of $P$. The modulo operation is easily calculated with a bitwise and-operation if $P$ is a power of 2 . As soon as memory pointers and memory step registers are set (the configuration component), the instruction word provides the necessary information about how to update 
the address pointers (the instruction component) and which permutation vector or table to apply (the LUT-component).

\subsubsection{AGU features}

The previous example demonstrates several AGU requirements to enable orthogonal computing/addressing with compact permutation tables. The design of a proper AGU is a complex optimization problem with many factors to consider: hardware cost, instruction word length, permutation table size, total code size, application/kernel requirements, generality/flexibility and energy consumption. It also effects how often HOF execution must be terminated unnecessarily, because some certain address register update feature that would be useful is not available. Based on practical experience gained from kernel implementation, the following set of AGU features may be a reasonable trade-off for DSP kernels on a HOF-based architecture:

- Address registers: A set of general or special registers, which can be used to hold base addresses for memory addressing.

- Addressing table registers: A set of general or special registers, which can be used to address a table of addressing data, such as a permutation table.

- Addressing enhancement registers: A set of general or special registers, to enhance addressing capabilities. These can include step registers (for programmable address register update steps) or top and bottom registers for modulo addressing.

- Every-cycle address register update: Address register update functionality, applied every clock cycle. Typical operations include incrementing one word (data type dependent), incrementing one vector $(=P)$, incrementing by a programmable step, fetch a step size from a LUT of steps or no update.

- Block-level address register update: Address register update functionality, applied after each HOF-block. The most common opera- 
tions are to increment the address register by a programmable step or perform no update.

- Every-cycle permutation vector retrieval: Retrieve and apply a permutation vector every cycle.

- Every-cycle permutation table address update: Modify the permutation table address register. Typical updates include incrementation to the next vector or no update.

- Modulo addressing: A table of steps or table of permutation vectors require circular addressing.

- Fixed address register offset: A fixed value supplied by an instruction, which offsets the base address or permutation table pointer.

A block diagram of an AGU supporting the proposed features is shown in Figure 8.11.

To reduce configuration overhead and reduce the number of permutation vectors required, one can additionally consider implementing the following features:

- Common base updates: Incrementing an address register by 1 (or possibly the size of a larger data type) or $P$ is very common and may be supported directly by the hardware, to avoid the configuration of a specific step register.

- Common permutation vectors: The most common permutation vectors are the "normalized-duplicated-scalar" (such as $[0,0,0, \ldots$, $0])$ and "normalized-consecutive" $([0,1,2, \ldots])$ permutation vectors, which may be supported directly by the hardware.

By introducing these features, many kernels can be implemented without requiring any explicitly stored permutation vectors or base update values, which frees them from all AGU configuration overhead, except for the address register setup.

By allowing address tables or permutation tables to be fetched from a register file, we make sure that we can support general addressing. The 


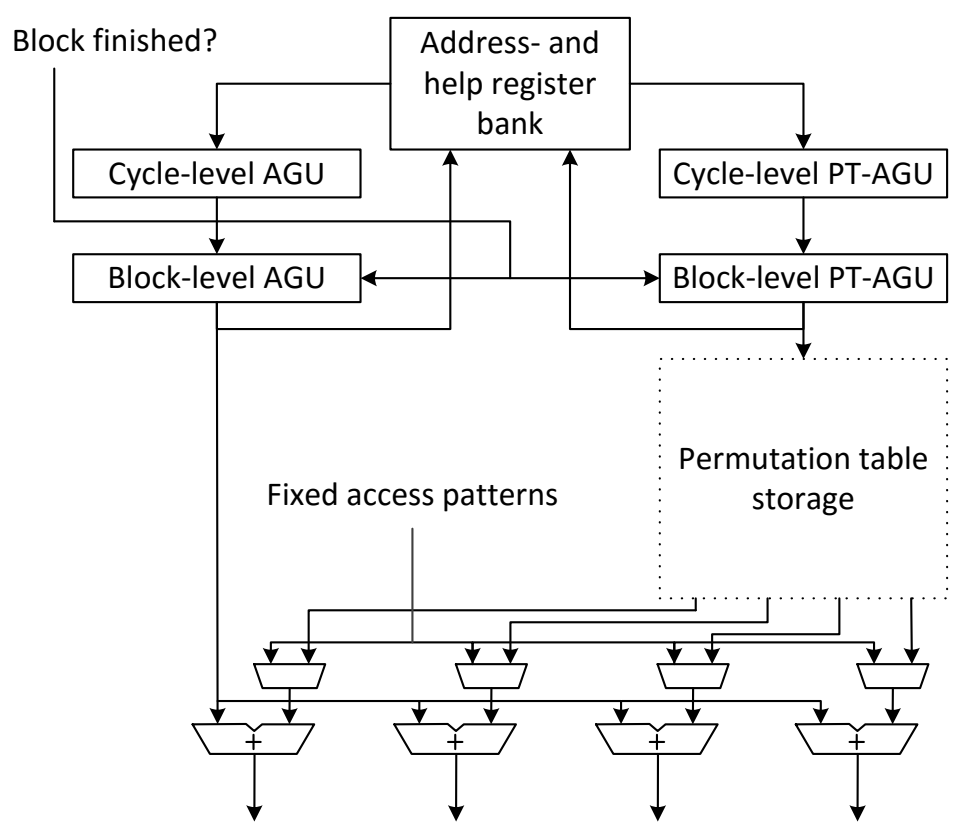

Figure 8.11: A block diagram of possible address generation circuitry for a HOF architecture.

remaining features are used simply to eliminate execution time overhead of addressing, reduce permutation table size and prolong possible $\mathrm{HOF}$ execution.

\subsection{Conflict-free unpredictable memory access}

So far we've discussed data access patterns that can be determined statically, in which case we organize our data to minimize data access overhead. If we can guarantee that our access patterns are conflict-free, we not only achieve high performance, but can also simplify the hardware implementation and reduce memory cost.

In scenarios where it is non-trivial or perhaps impossible to guarantee conflict-free data access with the standard bank assignment, one may take more extreme measures to guarantee that the memory can be ac- 


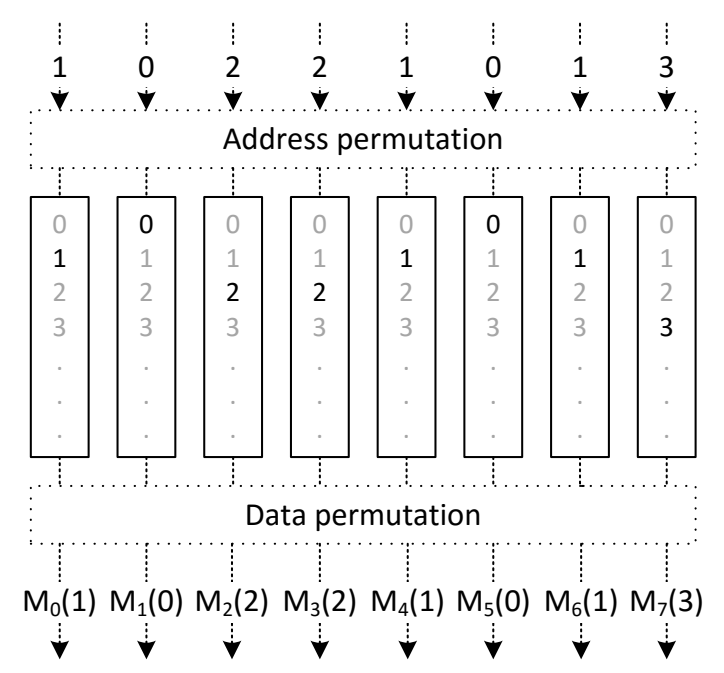

Figure 8.12: 8-way multi-bank memory divided into individual memory spaces for each processing lane.

cessed free from conflicts. This may happen in case the memory access pattern is based on input data one has no control over. Still, if we somehow can manipulate the distribution of unpredictable data to guarantee conflict-free access, we could potentially improve performance significantly.

The simplest solution to avoid potential bank conflicts for unpredictable access is to not use parallel memory access at all, but the performance is low. To improve on this, we may modify the bank assignment function (temporarily) to divide the memory space into multiple memory subspaces, e.g. one for every processing lane, as shown in Figure 8.12. The bank- and address assignment and functions are then changed into:

$$
\begin{array}{lr}
b_{i}=\Psi_{b}\left(\alpha_{i}\right)=i & \text { for } i \in\{0, \ldots, B-1\} \\
\beta_{i}=\Psi_{\beta}\left(\alpha_{i}\right)=\alpha_{i} & \text { for } \alpha_{i} \in\left\{0, \ldots, M_{b}-1\right\}
\end{array}
$$

where $M_{b}$ is the size of a memory bank. If the memory space is divided into as many partitions as there are memory banks, an access to address 
$\alpha$ by processing lane $i$ would be serviced by memory bank $i$ and no conflicts occur. This behavior can easily be implemented as a special memory addressing mode ${ }^{2}$.

The proposed bank assignment allows $P$-way reads and writes as long as we can work with $P$ unsynchronized memory spaces. This is useful for e.g. histogram generation, where one can accumulate data into $P$ separate vectors and then finally merge the $P$ sub-results. However, if the memory spaces must be kept in sync, every scalar written to memory must be distributed to all memory banks. This results in $P$-way reads and 1-way writes.

Consider that the number of memory banks is equal to the processing width $(B=P)$, and $W$ elements have been written to a memory buffer using the standard bank assignment function with no duplicated elements. We now want to perform $R$ unpredictable reads to this buffer. To guarantee conflict-free access we can either read 1 element at a time from the buffer, which takes at least $R$ cycles, or prepare the data for conflict-free access by duplicating elements ${ }^{3}$. Duplicating $W$ elements $P$ times takes at least $W$ cycles, but the read operations can then be performed in $\lceil R / P\rceil$ cycles. Due to pipelining some write latency $L_{w r}$ will be experienced before the first read can be performed. If we consider that we may choose between sequential read operations and parallel read operations with pre-processing, the overall lower bound on cycle time will be

$$
t_{\geq c y c, b e s t}=\min \left(t_{\geq c y c, s e q}, t_{\geq c y c, p a r}\right)
$$

\footnotetext{
${ }^{2}$ Note that the same behavior can be emulated by software under the standard bank assignment function, by simply performing the address calculation $\alpha_{\text {new }}=B * \alpha_{\text {old }}+i$. This however costs in terms of execution time overhead.

${ }^{3}$ Here we assume the worst-case, which is that the duplication of data has to be performed as a separate processing step. In practice, it may be possible to merge this duplication with the write operations of a previous processing step, to reduce or completely eliminate this overhead.
} 


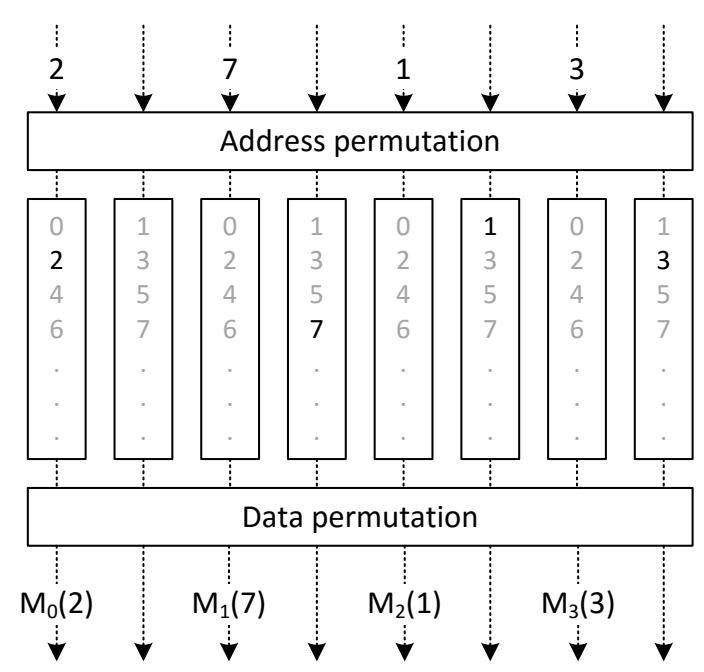

Figure 8.13: An 8-way multi-bank memory divided into 4 memory spaces. If the read operations are unpredictable and the memory spaces must be kept in sync, we can achieve 4-way read operations and 2-way write operations with this configuration.

where

$$
\begin{aligned}
& t_{\geq c y c, s e q}=R \\
& t_{\geq c y c, p a r}=W+L_{w r}+\lceil R / P\rceil
\end{aligned}
$$

For $R \leq W$, sequential memory access should always be a better choice, regardless of the values of $P$ and $L_{w r}$.

It is possible to improve the parallel performance. Instead of dividing the memory into $P$ memory spaces, we can divide it into $r$ memory spaces, with $r \leq P$, as shown in Figure 8.13. This means that we can only perform $r$-way reads, but since the write accesses are predictable we can improve write performance to $P / r$-way writes (assuming $P$ is a multiple of $r$ ), since data duplication is easily made conflict-free. This changes the lower cycle time bound for parallel data access to

$$
t_{\geq c y c, p a r}=\lceil W /(P / r)\rceil+L_{w r}+\lceil R / r\rceil .
$$


To determine the value of $r$ that minimizes total cycle time, we use the following approximation

$$
\begin{aligned}
& t_{\geq c y c, p a r}=\lceil W /(P / r)\rceil+L_{w r}+\lceil R / r\rceil \approx \\
& t_{\gtrsim c y c, p a r}=r W / P+L_{w r}+R / r
\end{aligned}
$$

and perform the following calculations:

$$
\begin{array}{r}
\frac{\partial}{\partial r}\left(r W / P+L_{w r}+R / r\right)=0 \Rightarrow \\
W / P-R / r^{2}=0 \Rightarrow \\
r=\sqrt{\frac{P R}{W}}
\end{array}
$$

In practice $r$ must be integer, but it should also evenly divide $P$. Since the derivative is monotonic, the optimum value under these constraints is (if not already a feasible value) the closest feasible integer smaller or larger than $r$, both of which must be tested. From hereon we call the best feasible value under these constraints $r_{\text {best }}$.

One of the most frequently occurring situations is the case when $R=$ $W$, from hereon called $N$. By using $r=P$ as originally suggested, sequential reading would always outperform parallel reading because of the overhead of data duplication. However, now we know that $r_{\text {best }}=$ $\sqrt{P}$, which results in a lower cycle time bound of

$$
t_{\gtrsim c y c, p a r}=\sqrt{P} N / P+L_{w r}+N / \sqrt{P}=2 N / \sqrt{P}+L_{w r} .
$$

Compared to the sequential execution time, we would like to know when $t_{\gtrsim c y c, p a r}<t_{\geq c y c, s e q}$, which is equivalent to

$$
\begin{aligned}
& 2 N / \sqrt{P}+L_{w r}<N \Rightarrow / P>4 / \Rightarrow \\
& N>\frac{L_{w r}}{(1-2 / \sqrt{P})} .
\end{aligned}
$$

This shows that it is possible to achieve the same or better performance using parallel memory access even in the case of $R=W$, although the processing width must be $P>4$. However, in general it is best if the ratio of $R / W$ is as large as possible, in which case the performance of parallel 
access with $r=P$ approaches the performance of predictable memory access patterns.

The relative speedup of parallel processing based on the ratio of $R / W$ for $P=16$ is compared to sequential processing in Figure 8.14. This plot assumes that $L_{w r}<<W$ or $L_{w r}<<R$ (or both), so that memory read time or write time totally dominates over $L_{w r}$. Two curves are shown, one which represents an architecture that only allows $r=P$ and the other representing an architecture which uses different memory addressing modes based on the $R / W$ ratio, and divides the memory space according to $r_{\text {best }}$. For small $R / W$ ratios, sequential processing performs best, because data preparation for parallel access dominates over read access time. For large $R / W, r_{\text {best }}=P$ and both architectures perform the same. The benefits of using $r_{\text {best }}$ show up when $R / W \sim 1$. This benefit increases with larger values of $P$.

\subsection{Related work}

Most modern processor designs with SIMD capability or similar, provide at least some mechanism to allow parallel processing on the datapath even in the presence of unaligned and out-of-order accesses. They can be divided into two main classes: register file-based parallel data access or memory-based parallel data access.

Architectures focusing on register file-based data access allow little flexibility for memory access $[22,51]$. Instead, data is first loaded from memory to a register file, where all data preparation is performed. These operations are typically more restricted than the proposed memory subsystem, because register file shuffles typically only allow data from two vector registers to be used as input, whereas multi-bank memory allow data from an entire memory to be fetched. This potentially leads to many shuffle operations before the data preparation is complete, unnecessary data may be fetched, more instructions are fetched, and execution time is prolonged. If the shuffling pattern must be placed adjacent to the shuffle instruction, there is less potential to reuse common permutation vec- 


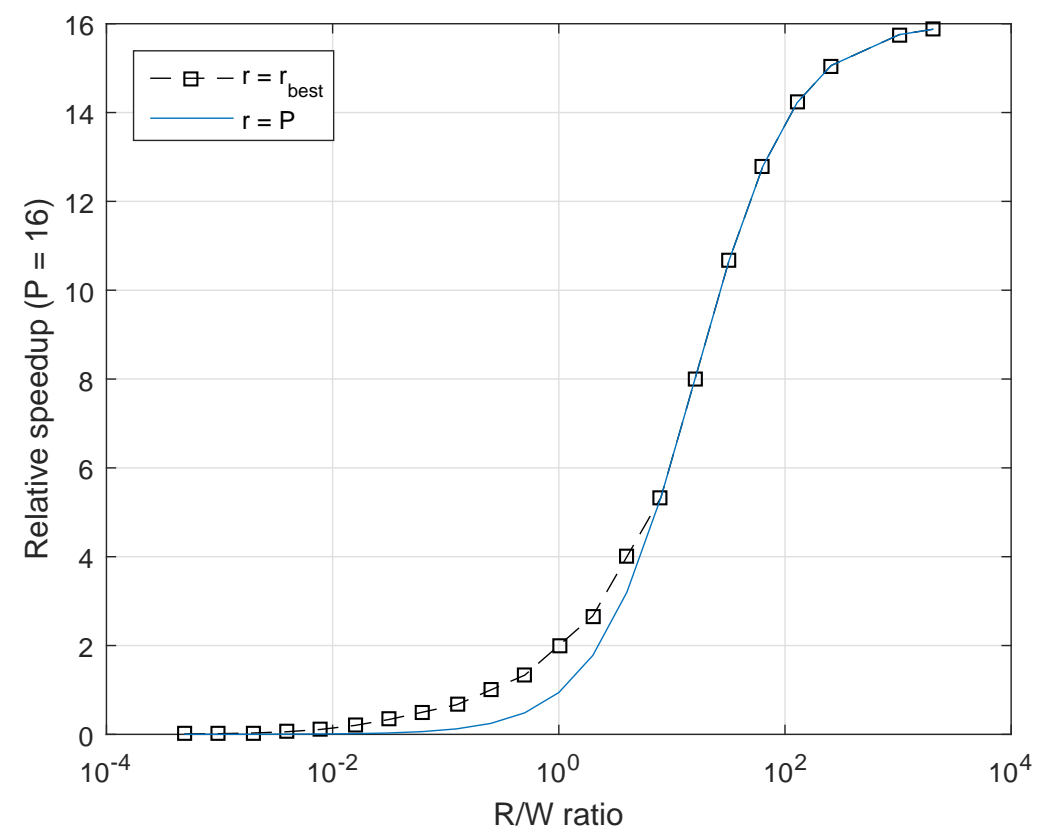

Figure 8.14: Relative speedup based on the $R / W$-ratio compared to sequential memory access for $P=16$.

tors/tables, which increases code size unnecessarily.

Similarly to multi-bank memory, it is possible to divide a register file into multiple banks. Several works focus on dividing a larger register file into smaller parts or introduce a cache hierarchy to the register file, in order to improve IPC, reduce latency or reduce the number of read and write ports $[82,83]$. These designs are not targeting vector access explicitly, but rather try to support many (unrelated) scalar accesses. However, there are examples of improvements for vector access, such as permutation vector registers which implicitly apply a permutation with each access to the register, potentially reducing shuffling overhead [84]. Reducing the number of required shuffle instructions has also been investigated by others [85].

Since the advantages of multi-bank memory can be quite significant, 
this feature is nowadays common in many types of architectures, including GPUs [73], general purpose DSPs [58], application-specific DSPs [60] and special-purpose hardware. Some architectures, such as GPUs and several general-purpose DSPs, primarily generate memory addresses using processor instructions and handles potential memory conflicts automatically, but with degraded performance. This results in more expensive hardware solutions with increased instruction fetching requirements. Our solution is to base data access on lookup-tables, which increases data fetching, but reduces instruction fetching, which potentially removes execution time overhead and reduces energy consumption. As we shall see in the next chapter, the fetching of permutation vectors can most often be completely hidden with cheap hardware solutions, at least in the DSP domain.

Application-specific DSPs on the other hand may take a different approach. By having full knowledge of the application at design time, the processor can be designed to support only the exact addressing patterns necessary for the application. This improves efficiency at the cost of generality. Generating fixed permutation datapaths for streaming data has been discussed by others $[86,87]$. These works sometimes only consider pure permutations: selection or duplication is not allowed.

The special addressing needs in DSP processing is also a well-studied subject $[88,89]$. However, the introduction of HOF instructions introduces more complexity to the address generation, because of their twodimensional nature. A slight increase in AGU complexity may in this case have significant effects on instruction fetching, and since fetching and decoding is typically much more energy consuming than address generation, we expect this increase in AGU complexity to be greatly offset by the benefits for instruction delivery. This will be investigated in later chapters. 


\subsection{Summary}

This chapter discussed the design of memory subsystems for conflictfree parallel memory access, with a special focus on multi-bank memory and their AGUs. The proposed memory subsystem and address generation circuitry is scalable to arbitrary processing width and bank granularities, and the cost of different bank granularities were estimated. We further investigated the memory requirements for different DSP kernels, in order to determine a reasonable level of memory parallelism. Finally, the special address generation requirements for HOF instructions were discussed and opportunities for performance improvement of unpredictable data access patterns were investigated. 


\section{Chapter 9}

\section{Handling the complexities}

\section{of storage and access of}

persistent data

The previous chapter addressed the access of dynamic data: that is the input, output and intermediate computing data of compute kernels. This chapter will instead be devoted to persistent data: data that remain constant during kernel execution. As already discussed, many kernels will require access to constants for their implementation. In addition, the access of dynamic data may require access to permutation vectors which must be stored and retrieved efficiently. In the worst case we need access to multiple vectors of constants/permutations at the same time. This chapter will investigate the usage of persistent data in actual kernels and compare different implementation strategies. The goal is to be able to support the most commonly occurring persistent data access patterns, with little or no execution time penalty, while still keeping the hardware cost low. 


\subsection{Problem definition}

The persistent data of a kernel can be divided into the three following categories:

- Kernel binary: The binary code of the compute kernel.

- Permutation tables: Permutation tables of statically known memory access patterns ${ }^{1}$.

- Kernel constants: Other constant data that is not permutation tables, such as pre-calculated data lookup-tables, FFT twiddle factors or filter coefficients.

In this context, persistent data is the data a kernel uses that doesn't change during kernel execution. Note that it doesn't say anything about what happens with that data between kernel executions, nor does it say anything about what may happen with other data sharing the same data storage resource as an active kernel. However, if we require that an active kernel's persistent data remains constant during kernel execution, some hardware simplifications can be made. Instructions, constants and permutation vectors may be stored temporarily in processor internal registers and we don't have to track any updates to these values. It is enough if the old internal state is flushed when a new kernel is started.

Any kernel naturally requires a kernel binary, while permutation tables and constants are optional. However, in the worst-case, any instruction can theoretically access up to three dynamic memory operands, all of which may be out-of-order accesses that require permutation vectors. This would cause one instruction load and three permutation vector loads for a total of four persistent memory vector loads per clock cycle. In case a local dynamic memory access is replaced by a kernel constant load, the persistent data access pressure stays the same. This represents a very high data access load which must be dealt with.

\footnotetext{
${ }^{1}$ Permutation tables created dynamically during kernel execution are excluded. Such tables can be placed e.g. in a register file.
} 


\subsection{Motivation and goals}

The problem just described may at first seem like an architecture-specific problem. However, the need to be able to access instructions and constant data is universal and any architecture must principally be able to address memory based on the contents of a lookup-table. The main problem that we try to solve is thus not related to functional capability, but rather to performance. Specifically we would like to be able to approach the performance limit of an architecture as defined by our design goals from Section 7.2. Since our data access hardware should be able to deliver at least $2 P$ input elements per clock cycle to the datapath, and those accesses could require permutation vectors, permutation vector fetching and dynamic data fetching must in some sense overlap. This capability is beyond the scope of most other architectures. The goal of this chapter is thus to investigate what possible solutions we can apply and investigate their cost in terms of hardware. As we soon shall see, the possible solutions to this problem are very much dependent on the behavior of actual kernels.

\subsection{Related work}

Many architectures, such as the STI Cell, store all local data in a single memory and accept the potential memory bottleneck associated with it [51]. If the processor can only perform simpler operations, a register file can significantly off-load the memory subsystem. However, as soon as datapath capability increases, memory pressure will eventually also increase and something must be done to avoid a memory bottleneck.

Cache-based architectures typically implement a lowest-level cache separated into an instruction cache and a data cache. This is common in both general-purpose processors [90] and DSP processors [58]. Instructions reside in the instruction cache, while dynamic data is placed in the data cache. Other persistent data may end up in either the data cache or the instruction cache depending on how it is used. For these archi- 
tectures it makes perfect sense to separate instructions from other data, since they typically execute one or more instructions every clock cycle and the demands on instruction delivery are high.

Specialized architectures may introduce memories dedicated to specific purposes to partly or fully eliminate any potential memory access conflicts. By placing e.g. instructions, dynamic data and constants in different memories, one may even optimize the word size of each memory individually [60]. While we do not exclude this possibility in a final system, our main goal is to design a configurable base architecture which can be configured towards multiple applications and it is therefore important that our basic design is relatively general. We seek a general solution that covers the most commonly used data types.

Many architectures perform rearrangement operations using the register file, or alternatively, use specialized memory addressing modes designed for a particular algorithm or application. When a register file is used, the data access pattern is typically stored as an immediate operand or in an additional register. The most common implementations of register file shuffling are more limited than direct memory access to multibank memory. Also, if the access patterns are embedded as immediate values in the kernel code, it limits the reuse of access patterns. The experience gathered during the course of this research shows that many patterns reappear frequently, which is an excellent opportunity for persistent data reduction. Our goal is to take performance and energy efficiency further, by avoiding compute-time overheads for data access and avoid data duplicates.

In this context one may also optimize the retrieval of instructions. One approach is to introduce a smaller filter cache between the local store or level-1 cache and the instruction decoder, for the purpose of energy reduction [91]. It may even be placed after the instruction decoder, to avoid the decoding energy cost [92]. Another approach is to introduce loop caches [93]. Such caches only cache the code of loops, under the argument that such code is executed many times. A loop can be detected in several ways. One way is to start caching instructions as soon as a 
hardware repeat instruction is detected, since this certainly indicates a loop. Some architectures also assume that a loop has been detected as soon as a backward branch has been detected.

An alternative to caches is to use software-managed register files for instruction storage. This gives a higher level of control over instruction movement and energy benefits may be observed [29]. This requires that the tool-chain or programmer indicate which parts of the code will benefit the most from being loaded to temporary storage, but the overhead of cache hardware is eliminated. Our approach on the other hand tries to avoid the problem entirely, or at least significantly reduce it, by making a single instruction, a HOF instruction, self-sufficient for many cycles. Still, some kernels may not benefit as much from vector processing, which could make these techniques useful anyway.

\subsection{Kernel analysis}

The most straight-forward solution to the persistent data access problem is to duplicate all persistent data over multiple memories during kernel loading or to use multi-port memory. This would allow multiple simultaneous read operations, but the hardware cost is high. To reduce hardware cost, one may dedicate each memory to its specific purpose and decide the size of each memory based on kernel requirements. Unfortunately, different kernels have very different needs, which makes it hard to decide an appropriate size for each memory. An alternative is to let the software load constants or permutation vectors to some temporary location, e.g. a register file. However, this consumes additional time and may greatly reduce performance if new data has to be loaded frequently. In such a scenario, the usefulness of HOF instruction execution is also reduced. The key to designing an appropriate persistent data access architecture, is to determine the actual persistent data access behavior of real kernels.

As with a dynamic memory architecture, to design an appropriate memory architecture for persistent data, we must consider both the or- 
ganization of the memory or memories, as well as the required address generation capabilities. If some required persistent data is available in memory, but it cannot be addressed efficiently, one may be forced to duplicate data and organize the duplicate differently. A typical example is $2^{x}$-FFT. Each layer of FFT requires its own set of data access vectors and twiddle factors. If appropriate persistent data addressing is available, only the largest twiddle factor set from the worst-case layer has to be stored, because all required twiddle factors of the other layers are a subset of the twiddle factors required for the worst-case layer. However, to efficiently access the correct vectors, strided data access must be available. If our FFT implementation should support multiple sizes of FFT computation (such as several $2^{x}$-point FFTs) with the same twiddle factor set, the potential savings become even greater.

It is certainly attractive to be able to store all persistent data in a single memory, at least if it can be done without performance issues. First of all it means that a kernel (with its associated permutation vectors and constants) can be transferred from main memory to local storage as a single software package with a single memory transfer. Secondly, one can avoid sizing issues that show up when using multiple memories, which potentially leads to better memory utilization.

A simple classification, such as the one in Table 9.1, can be used to guide us through the design process. The classification is based how intensively a certain kernel needs access to a particular type of persistent data. In this classification the presence of HOF instructions (or similar vector instructions) is assumed. A kernel that primarily executes vector instructions may therefore have very low instruction fetching requirements, which would leave a potential instruction memory free for other purposes.

We can quickly identify four benign cases: Given the presence of vector instructions, operations which are often directly supported by hardware such as (long) vector/matrix addition/subtraction, $\min / \mathrm{max}$, absolute value, element-wise multiplication/division/reciprocal or transcendental functions, logical operations or shift operations, with consec- 
Table 9.1: Kernels divided into categories based on how intensively the kernel uses a certain type of persistent data.

\begin{tabular}{l|l}
\hline \hline Kernel category & Example kernels \\
\hline Non-intensive & $\begin{array}{l}\text { simple (long) vector/matrix } \\
\text { operations (add/sub, ...) }\end{array}$ \\
\hline Instruction-intensive & $\begin{array}{l}\text { traditional RISC/SIMD code, } \\
\text { short vector computing, } \\
\text { non-vectorizable code }\end{array}$ \\
\hline Constant data-intensive & filters (e.g. FIR) \\
\hline Permutation-intensive & data rearrangement, interleaving \\
\hline Constant\&perm.-intensive & $\begin{array}{l}\text { transforms (FFT, 2D-DCT, ...), } \\
\text { image operations, ... }\end{array}$ \\
\hline All-intensive & - \\
\hline \hline
\end{tabular}

utively addressed elements, can be classified as non-intensive, since a single instruction may execute for tens, hundreds or even thousands of clock cycles. The three remaining benign cases are cases when only a single type of persistent data stresses the memory. Traditional RISC code is typically very much instruction-intensive, but in case vector instructions are available there are often better ways to express the computation. Still, when vectors are very short or non-existing, kernels may become instruction-intensive. The remaining two cases are constant dataintensive kernels, such as filtering kernels which repeatedly apply filter coefficients, or permutation-intensive kernels, such as interleaving (which simply rearranges data) or kernels using permutations over small data blocks ${ }^{2}$.

Several important kernels use more than one persistent data source continuously. Examples include FFT, which requires both twiddle factors and permutation vectors for bit-reversed addressing or 2D image filter-

\footnotetext{
${ }^{2}$ Conflict-free access of data blocks using general permutation is possibly only useful for smaller blocks, since the cost of permutation tables for larger block sizes may be huge. In this case, padding is a better choice.
} 
ing, which requires both filter coefficients and possibly an access pattern to retrieve the correct pixels to filter. Fortunately, these kernels are easily vectorized and the number of permutation vectors can be made few, which makes it possible to store the permutation vectors in a register file or cache. This only leaves the constant coefficients, which in the case of FFT can be quite many. However, since the computations are easily vectorized and the permutation vectors are few, the memory is free to fetch twiddle factors and a single local store still seems to be enough.

Based on practical kernel implementations, the use of a single persistent data memory has turned out to rarely be an issue, as long as appropriate temporary storage for frequently accessed data is provided. The full list of kernels this analysis is based on is found in Appendix C. Temporary storage could be implemented either by introducing data caches, by reusing the general register file or by introducing special-purpose register files. The goal of the temporary storage is to make sure that any real-world kernel would very rarely need to access more than one vector from persistent data memory every clock cycle. The two following subsections will discuss the needs for permutation table access and constant data access separately.

\subsubsection{Permutation table access}

Chapter 8 discussed two different methods to achieve conflict-free access, and their persistent data usage differ to some extent. Kernels that extensively use padding often only require a single permutation vector [47] per vector instruction. For example, if a matrix has padded rows, to allow both row-wise and column-wise conflict-free access, rows can be accessed without any permutation vector, while a single permutation vector may be used to extract columns. In case padding is not satisfactory, one can turn to general permutation, which may improve dynamic memory usage density, but increases the size of permutation tables. In this case, it is typically beneficial to divide a larger data structure into blocks, blocks which have side lengths of the processing width $P$ or a multiple thereof $[46,8]$. 
Permutation vector usage behavior typically falls into one of the three following categories:

1. A single or a few vectors are repeatedly used (e.g. for padding).

2. 2D data blocks with side lengths of processing width $P$ are repeatedly accessed (e.g. block-wise permutation).

3. A very large set of permutation vectors are accessed consecutively (e.g. general interleaving).

Based on these patterns, a cache or explicitly managed buffer with $P^{2}$ entries will handle the two first cases perfectly. Permutation tables are most often accessed consecutively and repeatedly, meaning that some form of circular addressing is more or less mandatory. Fortunately, random accesses into permutation tables are very rare. For the last case, it is hard to draw a conclusion on how many permutation vectors are actually required, since this is highly application dependent. However, this situation is rare, and when it happens, the permutation vectors are often the only source of memory pressure, which means they may be fetched directly from memory without any performance issues.

\subsubsection{Constant data usage behavior}

Typical kernels access constant data in one of the four following ways:

1. An entire table of elements is accessed once. All elements are visited in a consecutive order, or alternatively using strided vector access.

2. Same as above, except that each element (or sub-vector of elements) is accessed multiple times before continuing with the next.

3. A table of elements is accessed circularly.

4. A table of elements is accessed randomly (table-lookup).

Alternative 1 gain little from temporary storage, since elements are only accessed once. Alterative 2 gain from a single-entry storage location, but 
otherwise the scenario is the same as alternative 1. Alternative 3 can benefit from temporary storage. The problem is that the entire buffer must fit in temporary storage for optimal results and the number of table entries is very kernel-dependent. Alternative 4 may benefit from temporary storage if elements are accessed multiple times, otherwise not. Performance becomes unpredictable.

Alternatives 1 and 2 are found in e.g. FFT. It seems that a single vector cache could be of good use for a very small hardware cost. Also it wouldn't introduce any unpredictable behavior in cache replacement, since there anyway is only a single entry to replace. Making the cache larger than a single entry can give very kernel-specific benefits, but it is hard in general to decide what a good size should be, since the actual need could vary from one to thousands of entries. A good default implementation seems to be a cache or temporary storage location with a single vector entry, with the possibility to extend it based on application needs.

\subsection{Explicit data management and cache-based solutions}

The kernel analysis provides some hints regarding an appropriate size for temporary storage of permutation vectors and constants, and how these data sets are typically addressed. However, it doesn't say how this temporary storage should be implemented. Perhaps most importantly, one has to decide whether to use a cache-based solution or explicitly managed buffers/register files. The following lists summarizes the most important advantages and disadvantages of these two alternatives:

\section{Explicitly managed memory buffers:}

+ Lower energy cost for access to already loaded data.

+ No cache hardware required.

+ Predictable behavior. 
+ Possibly higher performance for irregular access patterns.

- Lower performance, if the processor cannot issue buffer loading instructions in parallel with other compute instructions.

- Larger code size, due to explicit data loads.

- May require frequent explicit reloading for larger data sets.

- Higher instruction delivery energy, especially if long vector operations need new data frequently.

- Higher initial tool-chain implementation effort.

\section{Cache-based buffering:}

+ Memory handled automatically.

+ Higher performance, since data loads can be overlapped with computing.

+ Lower instruction delivery energy.

+ Simpler programming/code generation.

+ Better utilization of HOF instructions.

- Possibly lower performance for irregular access patterns.

- Higher hardware cost.

- Possibly higher energy cost to access cached data, due to cache overhead.

- Possibly unpredictable performance.

The choice may not be immediately clear. Explicitly managed buffers would suffer from lower performance, since buffer fills will consume clock cycles if we keep the design principle of maximally one active instruction at a time. As an example, a 256 point FFT would consume 33\% more cycles if twiddle factors cannot be loaded in parallel with butterfly computing (overheads excluded). HOF instructions may not execute for very long before buffer refill, in case the tables are large and data is not reused. In cases where every single entry is only used once, half of the execution time would be spent on buffer fills, which is certainly 
not attractive. This situation turns out to be common, which is a serious problem with the explicitly managed buffer approach. This is the main reason why this approach has been abandoned in this research.

If a cache is introduced, additional design choices must be made, such as cache line size, associativity and possibly replacement policy [94]. It makes sense to use a cache line size of a single vector to avoid unnecessary data loads and to avoid having to align data to a certain cache line boundary for best performance. The performance of a set associative or fully associative cache will depend on the replacement policy [95]. Based on the fact that permutation tables and constant data tables are often linearly or cyclically addressed, the most recently used (MRU) replacement policy could be a good choice. However, the kernels implemented so far have shown no benefit from a set associative cache. The simplicity and predictability of a direct-mapped cache is quite attractive and often sufficient.

\subsection{Multi-bank memory}

As with dynamic data, one should also investigate the potential benefits of using multi-bank memory. Although there may certainly be exceptions, the implemented library of kernels indicate that unaligned vector access to constants or permutation vectors is rarely useful. It is sufficient to be able to access aligned vectors or sub-vectors, such as aligned halfvectors or individual words. For these scenarios, multi-bank memory is not necessary, at least from a performance perspective.

For instructions, the situation could be different. If the processor uses variable length instructions, it may happen that an instruction spans two memory vectors, as shown in Figure 9.1(a). This is not a problem, except when instructions are not executed linearly, e.g. when a jump or call instruction is taken, or every time a hardware loop begins a new iteration. If the target instruction spans two vectors, an additional cycle may be spent to fetch the entire instruction before execution can continue. Unfortunately there are kernels with very small hardware loops, where this 


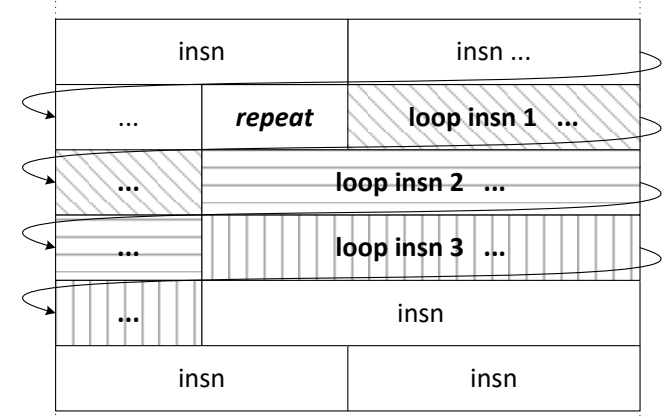

(a) Code with unaligned loop start.

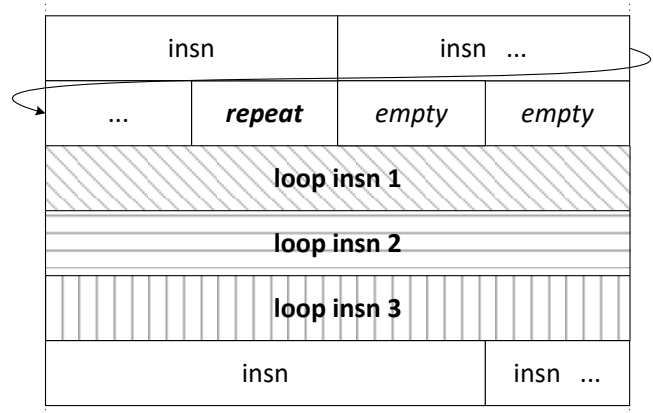

(b) Code with aligned loop start, padded with an empty instruction.

Figure 9.1: A comparison between an unaligned loop and a loop that has been aligned using an empty instruction. If all instructions are single-cycle instructions, the unaligned loop may consume 4 cycles per loop iteration, while the aligned loop will only cost 3 .

penalty could add $10-30 \%$ of extra execution time.

There are many possible solutions to this issue. One of the simplest, and possibly cheapest from a hardware perspective, is to align all jump target instructions that span two vectors in the kernel, by inserting filler instructions, here called empty instructions, as shown in Figure 9.1(b). Such an instruction performs no operation, but unlike a nop (no operation) instruction, it does not consume any execution time, as long as 
there is some other instruction in the instruction vector to process. Although this consumes some extra code space, it is significantly cheaper in terms of code size than loop unrolling, which does not eliminate the issue, but only reduces it. It is also cheaper in terms of hardware than inserting loop caches, increasing the instruction fetching bandwidth or adding multi-bank memory. The insertion of empty instructions is easily automated in an assembler.

\subsection{Pipeline organization}

So far we've concluded that a direct-mapped cache-based solution might be the best choice for access to constant data and permutation vectors. To support permutation and constant data access simultaneously, we introduce separate caches for constants and permutation vectors. Additionally, each dynamic memory port may require permutation vectors, which means that we also need multiple permutation vectors caches. All persistent data is stored in a single local store and the caches are filled as needed.

The local store is generally a potential source for hardware conflicts, and it is important to decide how this resource should be used. Quite naturally, an instruction must first be fetched before its requirements of permutation vectors and constants are known. Furthermore, the permutation vectors must be available before dynamic data memory can be accessed. To implement a streaming computation flow for HOF instructions, these precedence constraints can result in a processor pipeline structure as the one shown in Figure 9.2. The local store provides instructions to the instruction decoder, permutation vectors for up to two source operands and possibly constant data as one operand. After any potential permutation vectors are read, dynamic memory is read, the data is operated on in a datapath and finally written to a destination location. The destination may also require a write-back permutation vector. Each time a permutation vector or constant data vector is read from the local store, it is also cached. 


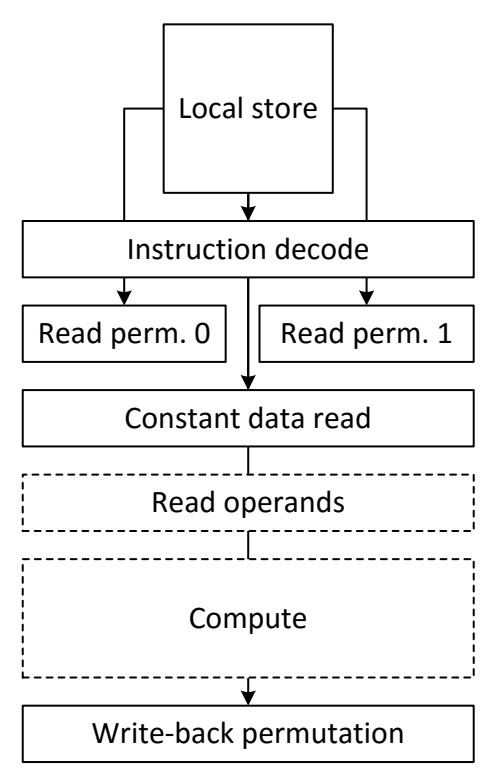

Figure 9.2: Persistent data memory usage in a possible HOF-processor pipeline.

If the actual reads of the local store are organized in time as shown in Figure 9.2, a single iteration of an instruction ${ }^{3}$ may read a source permutation vector, a write-back permutation vector and a vector of constants without any performance reduction, since the memory reads are distributed over multiple pipeline stages. Two read permutations may conflict for the use of the local store, if they are concurrent in time. However, two simultaneous read permutations are rare in practice, and if one of them is already cached, no performance degradation will be noticed anyway. Although a single instruction iteration rarely conflicts with itself, this organization means that it can conflict with other instruction iterations and other instructions.

The proposed pipeline organization can be implemented in such a way that as long as only a single memory access is required at any time,

\footnotetext{
3"Single iteration of an instruction" refers to the fact that HOF instructions are multicycle instructions. A single instruction iteration refers to a $P$-way chunk of the total work of the instruction.
} 


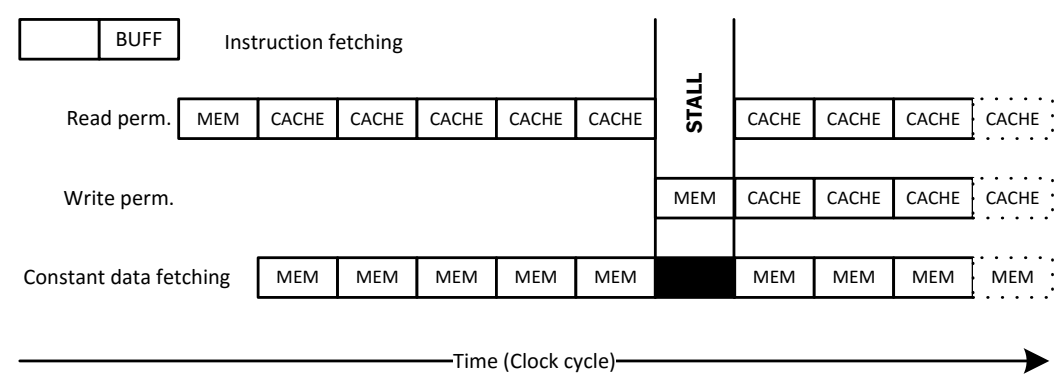

Figure 9.3: Persistent data memory usage over time. The introduction of small caches for permutation vectors and constants can greatly reduce memory access conflicts. Cycles that would otherwise be idle are best spent on buffering more instructions.

no performance penalties will be noticed. Additionally, there is no performance difference between a cache-hit or cache-miss, as long as cachemisses do not lead to multiple simultaneous memory access requests. Consider a multi-cycle HOF instruction using one permutation vector for memory read and one for memory write, a typical scenario when using padding. Additionally, every cycle we need to access a new unique vector of constants. This situation shows up in e.g. FFT computing. The use of the persistent memory subsystem may then look like the Gantt chart in Figure 9.3. The read permutation vector is read and cached before constants are fetched. When the write-back permutation vector is required, the access of constants has to be paused and one processor cycle is lost. However, after this point, no more slow-downs will affect the instruction. Any memory idle time may be used to buffer more instructions. Also, since we assume that the data is persistent, there are no issues with memory write-back when cache lines are evicted.

\subsection{Evaluation}

Since we have not yet introduced a practical HOF-based processor, we shall evaluate the proposed persistent data memory architecture in general terms based on what we expect from a real-world kernel implemen- 
tation. In addition we include an all-intensive synthetic worst-case kernel for comparison. In general it is simple to implement a real-world kernel that triggers worst-case behavior as well, by simply accessing dynamic memory data using large tables of unnecessary permutation vectors and avoid using HOF functionality. However, we assume that the kernels are well implemented.

Table 9.2 lists some typical kernels that we will consider. This table indicates whether or not multi-cycle HOF instructions can be used to implement the main work of the computation. Merge sort is one of the few examples where this is not possible. This is because data access depends on input data. After a merge operation has been performed, data dependent pointer updates must be performed, a computing pattern not directly supported by a single HOF instruction. This makes this kernel instruction-intensive. The table also indicates the use of permutation vectors and constant data, where Few indicates that the kernel may be implemented with so few persistent data vectors of the given type that they fit entirely in a typical $P$-entry cache. Many indicates the opposite, a $P$ entry cache is too small to hold all required vectors. For some kernels the number of permutation vectors or constants depends on problem size, which is indicated by Few-Many.

In addition to the persistent data usage, Table 9.2 also indicates what cycle time overhead one can expect by using the proposed memory architecture compared to an optimal architecture were no conflicts ever occur. The actual overhead depends on many factors, such as processing width, instruction set and what method for conflict-free access is used, so this value represents an estimate. It turns out that for most practical kernels, we expect no cycle time increase at all, because the kernel maximally requires a single source of persistent data or multiple sources with so low intensity that they will not conflict in a processor pipeline. For kernels that may expect some overhead, the overhead depends on the problem size $N_{i}$. It is however important to note that many of these kernels are typically used to process many blocks of data at the same time, and the number of blocks to process $N_{o}$ doesn't affect the cycle time overhead, 
Table 9.2: Cycle time penalty when using the proposed persistent data memory architecture instead of an optimal one for some typical compute kernels. Note that most kernels have a fixed overhead, independent of how many blocks to process, since some or all persistent data vectors may be cached after the first block has been processed. Few and Many in this context refers to whether or not the number of static data vectors are few enough or too many to fit entirely in a typical cache-setup of proposed size.

\begin{tabular}{|c|c|c|c|c|}
\hline Kernel & 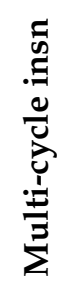 & 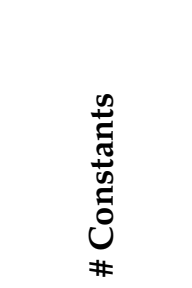 & 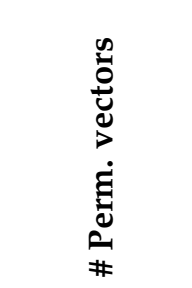 & 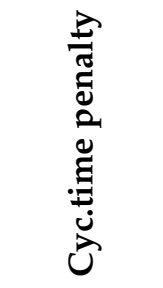 \\
\hline Simple element-wise & Yes & - & - & None \\
\hline$N_{i}$-point FFT & Yes & Many & Few & $\sim \log _{4} N_{i}$ \\
\hline Matrix ${ }^{*}$ Matrix & Yes & - & - & None \\
\hline Matrix ${ }^{*}$ Const matrix & Yes & Few-Many & - & None \\
\hline Matrix (QRD/LUD/inv) & Yes & - & $($ Few $)$ & None \\
\hline Sparse matrix * vector & Yes & - & - & None \\
\hline Matrix transpose & Yes & - & Few & None \\
\hline FIR-filter & Yes & Few-Many & - & None \\
\hline$N_{i} \times N_{i}$ 2D-DCT & Yes & Few & Few & $\sim N_{i}^{2} / P$ \\
\hline Bitonic sort & Yes & - & Few & None \\
\hline Merge sort & No & - & - & None \\
\hline Histogram generation & No & - & - & None \\
\hline Median filtering & Yes & - & Few & None \\
\hline Interleaving & Yes & - & Few-Many & None \\
\hline Function (e.g. Taylor) & Yes & Few-Many & - & None \\
\hline Morphological ops. & Yes & & Few & None \\
\hline$N_{i} \times N_{i} 2$ D convolution & Yes & Few & Few & $\sim N_{i}^{2} / P$ \\
\hline Worst case (synthetic) & No & Many & 2xMany & $3 N_{i} N_{o} / P$ \\
\hline
\end{tabular}


because of caching. There are bound to be exceptions to this, but among these examples the only exception is the worst case synthetic kernel.

Table 9.3 compares the relative characteristics of a few discussed memory architectures. This analysis assumes that a typical cache consumes a few percent of the area of memory and that a cache read access costs $10 \%$ of the energy of a memory access, values which are based on a typical architecture configuration and memory data sheet data. The single memory architecture has no temporary storage for permutation vectors or constants, which means that an instruction may require up to 4 cycles every time it is executed. No caching occurs. The dedicated memory architecture has 4 dedicated memories, possibly individually sized for some specific purpose, meaning that the area depends quite heavily on configuration. In general however, this solution has a very high hardware cost and tends to be 3-4 times larger than a single memory, but access conflicts are completely eliminated. Energy consumption relative to the single memory architecture depends on memory configuration, since smaller memories may cost less to access, but since some kernels require quite large memories for constants, the number of memory accesses and access energy tend to be similar to the single memory architecture.

The two final architectures use smaller register files or caches respectively as temporary storage. Due to the heavy spatial and temporal locality of permutation vectors and constants demonstrated in this chapter, the performance reaches similar levels to the dedicated memory approach for practical kernels. Because of the small buffers required, the memory area cost is not much higher than that of a single memory. Energy consumption can be significantly reduced, because of cheaper accesses to the smaller buffers. This rough comparison is not able to distinguish between an approach with explicitly managed buffers and a cache-based approach. The difference in area is negligible due to the very small simple cache implementation. The performance is similar if data is reused many times. The drawbacks of the explicitly managed buffer approach are primarily an increased code size and lower performance if data reloading is frequent, as discussed in Section 9.5. 
Table 9.3: Approximate relative characteristics of a few different possible persistent data memory architectures. The ranges represent the fact the actual value depends on memory configuration, but also the fact that the values depends on the executed kernel, since some kernels doesn't use permutation vectors or constants and therefore do not gain anything from more elaborate memory configurations.

\begin{tabular}{l|l|l|l}
\hline \hline Implementation & Area & Rel. speed-up & Energy \\
\hline Single memory arch. & 1 & 1 & 1 \\
Dedicated memory arch. & $1.1-4$ & $1-4$ & $\sim 1$ \\
Explicitly managed buffer arch. & 1.1 & $1-\sim 4$ & $0.1-1$ \\
Proposed cache-based arch. & 1.1 & $1-\sim 4$ & $0.1-1$ \\
\hline \hline
\end{tabular}

\subsection{Summary}

This chapter evaluated the design possibilities for a persistent data memory subsystem. The main purpose was to avoid the bottlenecks of a single memory, and solve the issue of retrieving constants and permutation vectors with very low execution time overhead, while still keeping the hardware cost low. Real-world kernels were analyzed in terms of persistent data access behavior. The analysis showed that most kernels can use small sets of permutation vectors or constants with extreme spatialand temporal locality, which makes a single persistent data memory with small caches for permutation vectors and constants an acceptable compromise between cost and performance. For kernels that do not behave in this way, there is typically only a single source of persistent data pressure at a time and continuous loading from memory is feasible without performance loss. 


\section{Chapter 10}

\section{Datapath design and optimization}

This chapter will discuss the design and implementation of parallel datapaths suitable for HOF architectures. HOF instructions move beyond the performance of SIMD instructions, principally by allowing the datapath to implement any operation to the set of all input data, as long as the instruction belongs to any of the previously described HOF computing patterns. We may exploit both datapath width, datapath depth and internal state, to maximally accelerate any general- or application-specific computing need. The implementation of such instructions may lead to complex networks of arithmetic units which we would like to reutilize for as many purposes as possible. At the same time, hardware cost must be kept low and compatibility with a possibly already existing kernel library and tool-chain may be required. A significant portion of this chapter is spent on deriving an optimization model which can be used to minimize datapath network interconnect. Finally, we will also investigate the cost of reconfigurable datapaths. 


\subsection{Datapath requirements}

Whereas the previously proposed memory subsystem is general enough to support most data access patterns efficiently, the same may not be true for any datapath. The addition of a well-chosen application-specific instruction can easily improve performance by an order of magnitude in some situations. At the same time, the datapath is often the largest and most energy consuming piece of logic in a processor. Adding unnecessary instructions may introduce high cost.

When designing an application-specific datapath for a HOF-based architecture, many things must be considered:

- Input/output bandwidth: A $P$-way datapath should generally be able to accept $2 P$ input elements and generate $P$ output elements, to support the worst-case map-based patterns.

- Datapath depth: To support reduce-patterns at maximum performance, with binary reducers, the datapath depth must at least be $\log _{2} P$ arithmetic units. To support reductions larger than datapath width, some type of accumulator is also required, increasing the depth to $\log _{2}(P)+1$ arithmetic units.

- Data types: Which are the required data types of the application? The choice between data types of the same width is primarily a matter of datapath cost and energy efficiency, but choosing between data types of different widths will also affect processing performance and storage density.

- Application-specific instructions: What application-specific instructions should be added to the design? May they reuse the basic datapath hardware, or should they be implemented by their own dedicated hardware?

- Compiler compatibility: A configurable platform may include a compiler for the architecture. Although we may choose any instruction set for our architecture, if we want to be able to use the al- 
ready existing compiler without modifications, we may have to implement some instructions that the compiler expects to be available on any platform instance.

- Kernel/instruction set co-design: Any given kernel (e.g. from a pre-existing kernel library) may assume that some instructions are available, otherwise the kernel cannot function. A kernel library may have to provide alternative implementations depending on instruction availability. Similarly, if we consider creating a new kernel, the potential benefits of datapath additions should be investigated.

The general design space is very large and one may have to perform several iterations of implementation and benchmarking before the final design is decided [4].

\subsection{Designing an application-specific datapath}

The design of an application-specific datapath is primarily a matter of knowing the application. One approach to finding suitable instructions is to analyze already existing application code to find common or performance critical computation patterns [96], but even better performance can be achieved if the datapath and kernel code are co-designed. The performance requirements of the different kernel algorithms is possibly the most important aspect. A high-level basic application implementation using algorithm kernels, and possibly already existing kernel implementations from a kernel library, may be of great help in determining an appropriate datapath.

A configurable platform may be shipped with a basic datapath structure that can support many types of operations. To exemplify such a datapath, we're going to consider a datapath designed from the structure of a complex radix- 4 butterfly operation. It turns out that this structure can support many other common operations and is therefore a suitable basic structure. Furthermore, FFT performance is very important in 


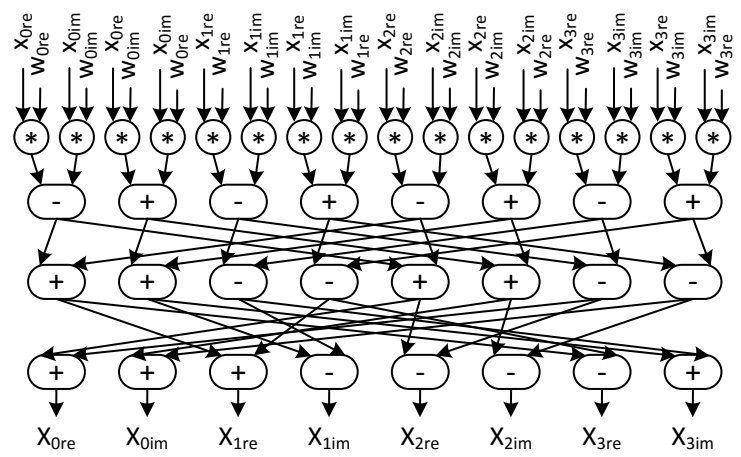

Figure 10.1: A datapath structure based on a complex radix-4 butterfly.

many applications and the butterfly structure is regular, has a suitable datapath width and depth, and is deep enough to support full reductions, if we add an additional layer of accumulation. The structure has been shown earlier, but is repeated in Figure 10.1 for convenience. Some computation structures in DSP can be implemented with pre-adders, but these are less common. Such operations could be implemented with multiple instructions.

If the adders/subtractors are generalized into more general arithmetic units, and we allow arbitrary interconnections between the layers of arithmetic units, we can implement many other operations using the same hardware. Figure 10.2(a) exemplifies a reduce-operation, a complex dot product. To allow vectors longer than the datapath width, two accumulators may be introduced, for the real- and imaginary parts respectively. By allowing min/max-operations in the arithmetic units, it is possible to implement a sorting network [97], as shown in Figure 10.2(b). Application-specific instructions, such as H.264 DCT shown in Figure 10.2(c), can also be added [78, 98]. This method of hardware multiplexing can significantly reduce overall datapath cost.

Depending on the actual data type supported by the multipliers and arithmetic units, it may also be possible to use the same hardware to implement operations on larger data types with the same hardware. If the multipliers are $n \times n$-bit fixed-point multipliers, $2 n \times 2 n$ multiplications 


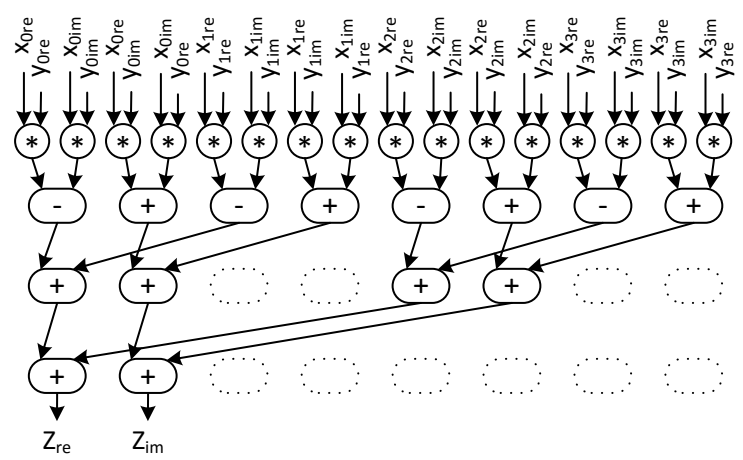

(a) Complex dot-product reduction.

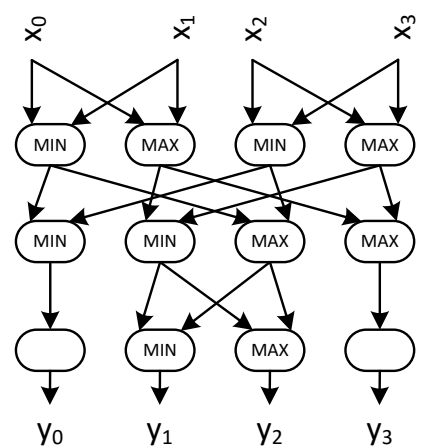

(b) A sorting network.

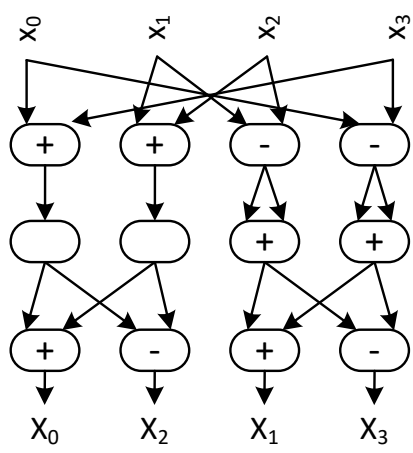

(c) H.264 Forward DCT.

Figure 10.2: The mapping of instructions to a datapath structure based on complex radix -4 butterfly operations.

can be implemented as shown in Figure 10.3. The same may not be true for other data types, such as floating-point values.

A complete generalized datapath structure that we will consider in this work is shown in Figure 10.4. The multipliers and arithmetic units operate on fixed-point values of some width. The sizes of multipliers and arithmetic units can be chosen so that no internal overflows can occur. Accumulators are introduced for all processing lanes, and typical DSP fixed-point post-processing features, such as scaling, rounding and saturation are added after the accumulators. Floating-point operations 


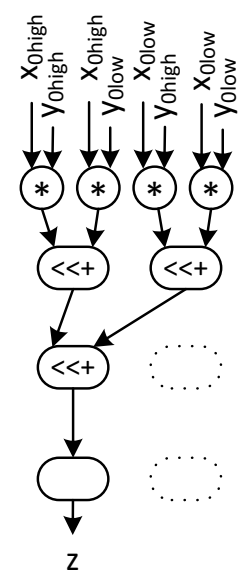

Figure 10.3: $2 n \times 2 n$-bit multiplication using $n \times n$-bit multipliers. The shift-andadd operation shifts one of the operands before addition.

can also be implemented on this structure, primarily by reusing the multipliers and arithmetic units and by adding some additional logic (not shown). Full IEEE-754 compatibility can be used if desired [99].

A configurable platform bundled with a pre-defined datapath structure may in some cases fulfill most of the processing requirements of an application. A platform designer may select a subset of the instructions from an already existing database of instructions that have been mapped to the pre-defined structure. In addition, one may add new instructions to the structure by simply forming new paths in the interconnect network between arithmetic units, or introduce a completely new additional datapath for some specific purpose.

\subsection{Datapath pipeline depth considerations}

The datapath pipeline depth is possibly one of the most critical design parameters. A longer pipeline depth may increase performance for some workloads, since it may allow the implementation of more complex datapath networks. Especially for long HOF execution, the pipeline depth 


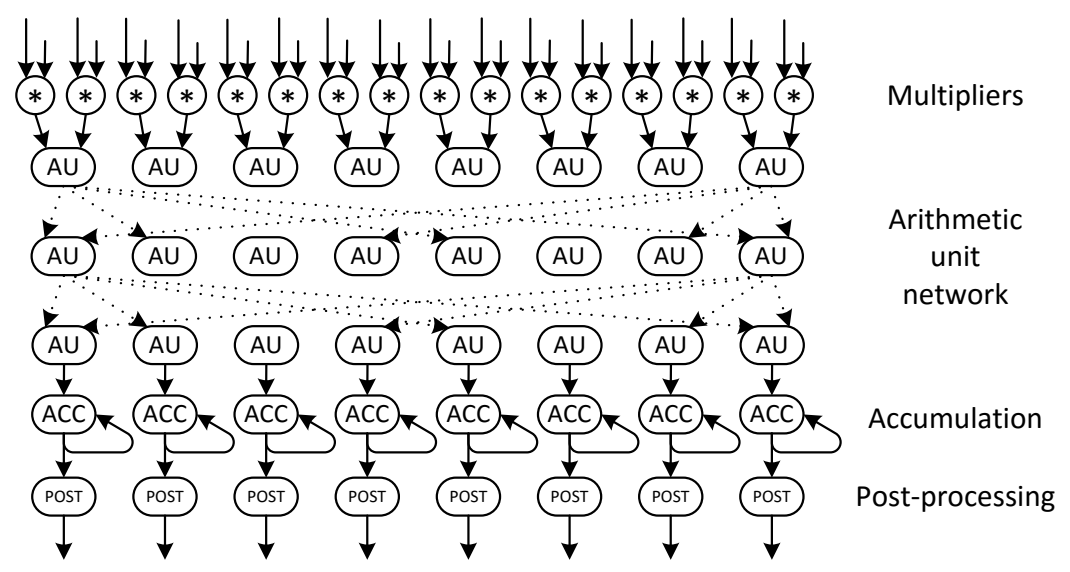

Figure 10.4: A complete datapath for fixed- and floating-point DSP processing.

has little effect on performance, but the potential increase in arithmetic units can increase performance significantly. The HOF design principle promotes the use of complex instructions and internalizing intermediate results within the processor's datapath, which may increase performance and energy efficiency significantly [30]. However, in cases where this is not useful, a large datapath depth may instead reduce performance. This can happen if we operate on short vectors, the operations are simple and there are data dependencies between instructions which cannot be internalized to the datapath. The problem can be counteracted in several ways, such as introducing multiple datapaths with different latencies, or by introducing bypass networks, at a cost of some additional result write-back circuitry. However, the problem is aggravated with increasing processing width $P$, if we want to keep full performance for e.g. reduce-operations.

Consider a case where $N$ elements should be reduced over a datapath of width $P$. A $P$-way datapath can be implemented as shown in Figure 10.5(a), and the vector may then be reduced over $\lceil N / P\rceil$ clock cycles. One may be forced to use this approach for some operators, however it results in a long critical path or long pipeline with $P$ sequentially con- 


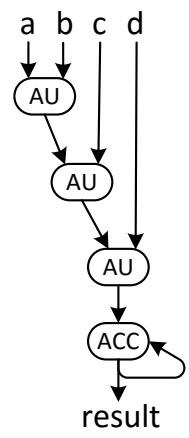

(a) Sequential reduction.

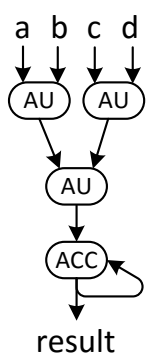

(b) Tree-based reduction.

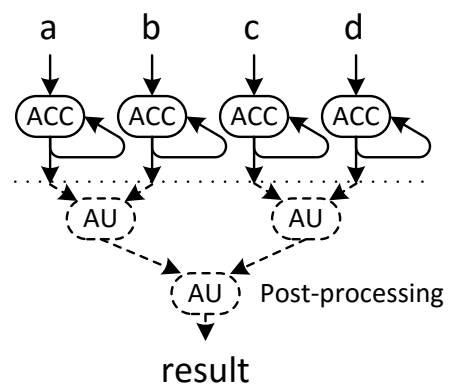

(c) Parallel reduction. Elements are reduced over multiple independent accumulators. The accumulators are finally combined in a post-processing step.

Figure 10.5: Three different reduction implementations.

nected arithmetic units (AUs). A better approach is to use the design in 10.5(b), which reduces the pipeline depth to $\log _{2}(P)+1$ AUs. A third alternative is shown in Figure 10.5(c), in which case $P$ parallel accumulators is used. Each datapath lane accumulates its own sub-results. After the input vector has been reduced, the accumulators can be combined in a post-processing step, which can be performed with the already existing hardware. In this case, the pipeline depth is short, but the lower bound on cycle count increases to $\lceil N / P\rceil+\left\lceil\log _{2}(P)\right\rceil$. 
Our example datapath from Figure 10.4 has a datapath depth suitable to implement tree-based reductions for many operations. This is preferred over parallel reduction, because of the higher performance. Additionally, we have already seen several examples of other types of instructions that can exploit this datapath depth. Some operations, such as floating-point addition/subtraction reduction, may instead be implemented using parallel reduction. This is because the additional complexity of floating-point operations may increase the datapath's critical path. Strictly speaking, many floating-point operations are not even associative [99], but in this context we often prioritize performance over possible round-off and cancellation effects.

\subsection{Optimizing arithmetic unit networks}

The implementation of datapaths with complex networks of arithmetic units leads to an optimization problem regarding instruction mapping. The overall design goal of a datapath is to minimize energy consumption and hardware cost under some performance constraints. A firstorder approximation of these quantities that we can use as a simplified optimization objective, is the number of links in the interconnect network and possibly also what operations each arithmetic unit can actually perform. This section will present an integer linear programming (ILP) model to address the issue [100]. It is not only applicable to processor datapaths, but also to similar processing pipelines in dedicated hardware implementations.

Before presenting the ILP model, there are several related subjects that should be mentioned. Lots of research has been spent on methods that try to find suitable custom instruction candidates for a given application, possibly directly from the application's source code [101, 102, 103, 27]. The focus of these works is however not the cost of the hardware, but rather to find suitable instructions that would improve e.g. performance the most. In [104] the focus is optimizing functional unit multiplexing, but the cost of interconnect is not considered. In [105] interconnect costs 


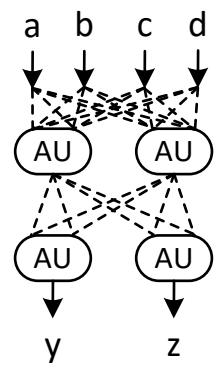

Figure 10.6: A small datapath pipeline and the potential interconnect paths.

are considered, but the logical datapath structure is fixed. The focus is instead to minimize the hardware of the fixed structure, by e.g. finding shared path segments. All of these topics are of interest to platform design in general, but not the focus of the optimization model that now will be presented.

We introduce our ILP model by considering a simple example: Consider a datapath with 2 input data vectors of 2 elements and 1 output data vector of 2 elements, as shown in Figure 10.6. We'll denote the 4 individual input elements as $a, b, c, d$ and the outputs $y, z$. The datapath consists of a pipeline of 2 layers of arithmetic units, each with 2 arithmetic units (AUs). Each arithmetic unit has 2 operand inputs and a single output. An arithmetic unit has been designed so that it can perform addition, subtraction, minimum, maximum, absolute value or simple bypass (no operation). Multiplication with 2 can be performed by doing $x+x=2 x^{1}$. We want to map the three following instructions to the datapath:

- INSN1: $y=a+b+c+d, z=a+b-(c+d)$

- INSN2: $y=\min (a, 2 b), z=\max (a, 2 b)$

- INSN3: $y=|a|+b+c, z=|a|-(b+c)$

A possible mapping of datapath operation INSN1 is shown in Figure 10.7(a). It introduces at least 8 links in the datapath pipeline, regardless

\footnotetext{
${ }^{1}$ Depending on the number format, there may be better ways to multiply with 2 . This only serves as a simple example.
} 
of mapping. We now map INSN2 and try to reuse the already existing links introduced by the first instruction. Two alternatives of equal cost in terms of added links are shown in Figure 10.7(b) and Figure 10.7(c). One arithmetic unit has to perform $2 b$, which leaves the other arithmetic unit to pass on the value of $a$ in the first layer. Regardless of which operation is mapped to which arithmetic unit, a minimum of two new links are required. Now, if we map INSN3 based on the two alternative mappings of INSN2, we can map it as shown in Figure 10.7(d). Based on mapping INSN2 - alternative 1 we need to add one more link to be able to perform $b+c$. Mapping INSN3 based on INSN2 - alternative 2 requires no new links, because the earlier choice already introduced the necessary links. The links of the two alternative complete datapaths are shown in Figure 10.7(e) and Figure 10.7(f).

Iterative mapping clearly doesn't guarantee optimal results, at least not in terms of the number of interconnect paths. It is hard to predict what effect each of multiple possible mappings will have on later instruction mappings. Instead, we are interested in finding a global optimum. Note that in many circumstances, finding an optimum solution to an optimization problem is not feasible, because the required run-time must be in the order of seconds or milliseconds. However, a datapath is possibly only generated once and then shipped in millions of copies. Optimization run-time is much less of an issue and even finding a $1 \%$ datapath area reduction may lead to significant cost savings. Even though we may not be able to find an optimal solution in reasonable time, we can typically spend much more time on trying to find it.

\subsubsection{General problem formulation}

We will now formulate the problem in more general terms. Consider an array of pipelined arithmetic units with a pipeline depth of $L$ arithmetic units. Further assume that each arithmetic unit can perform both unary and binary operations. In the case of unary operations, the unary operation can be performed on any of the two operand ports to the arithmetic unit. By default we also assume that all binary operations are commu- 

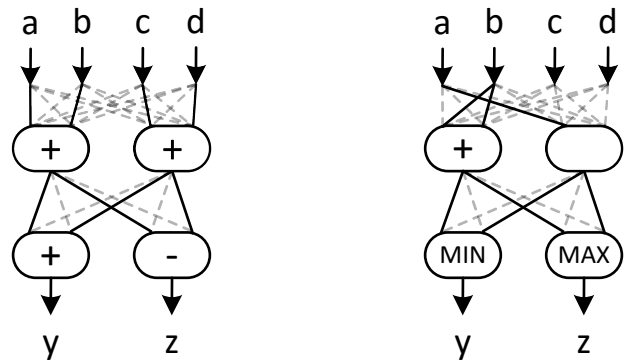

(a) Possible mapping of INSN1. (b) Possible mapping of INSN2 alternative 1 .
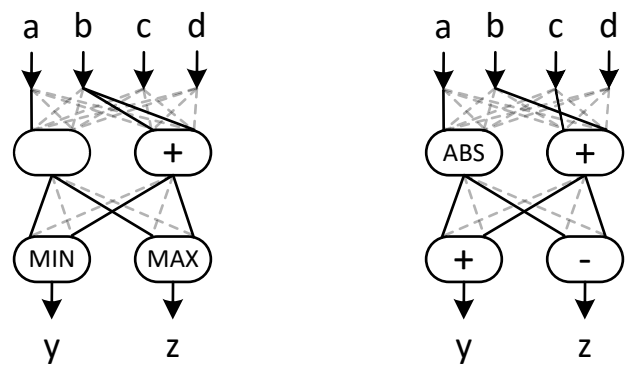

(c) Possible mapping of INSN2 - (d) Possible mapping of INSN3. alternative 2 .
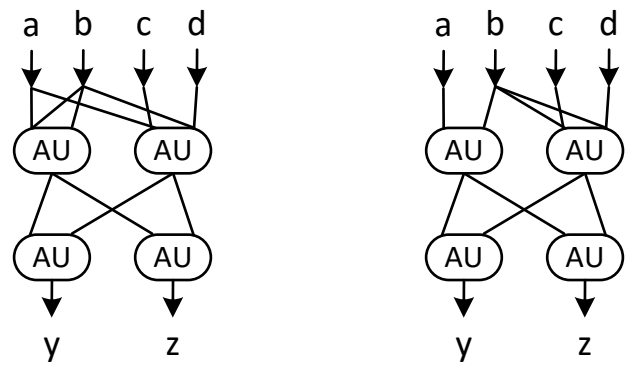

(e) A complete datapath based (f) A complete datapath based on the mapping alternative 1 of on the mapping alternative 2 of INSN2.

INSN2.

Figure 10.7: The mapping of instructions to a given datapath structure. 
tative. This covers many interesting binary operations, such as addition and minimum/maximum. Some operations, such as subtraction are not commutative, since $a-b \neq b-a$. To handle such operations we instead assume that an arithmetic unit can perform both $a \oplus b$ and $b \oplus a$. This behavior can easily be restricted if desired.

We introduce the two following ILP model variables:

- Paths $\Gamma_{l s d p}$ : The set of all possible interconnect paths in the interconnection network. The variables are binary variables, where a value of 1 means that the path should be included in the final datapath, and 0 indicates exclusion. The subscript $l$ indicates the pipeline layer, $s$ indicates the source (the datapath input or any previous arithmetic unit in the pipeline), $d$ indicates the destination arithmetic unit and $p$ indicates the exact input port on the arithmetic unit.

- Operation mapping $\mathcal{O}_{\text {iluo }}$ : Defines which arithmetic operation is mapped to which arithmetic unit for all instructions. The instructions that should be mapped to the datapath are first given a unique index $i$. Each instruction defines operations to perform in every pipeline stage $l$. For each layer, the operations to be mapped are enumerated. If operation $o$ of instruction $i$ is mapped to arithmetic unit $u$, in pipeline stage $l, \mathcal{O}_{\text {iluo }}$ is set to 1 , otherwise 0 .

The general problem setup is shown in Figure 10.8. Note that we do not introduce any restrictions on how many inputs or outputs exist, how many arithmetic units there are in each layer or similar. However, we do assume that one possible mapping of an instruction exists that can be used when building the optimization model, so that all individual operations can be identified and enumerated. As an example, if instruction INSN1 from our previous example is used, which was

$$
y=a+b+c+d \quad z=a+b-(c+d)
$$




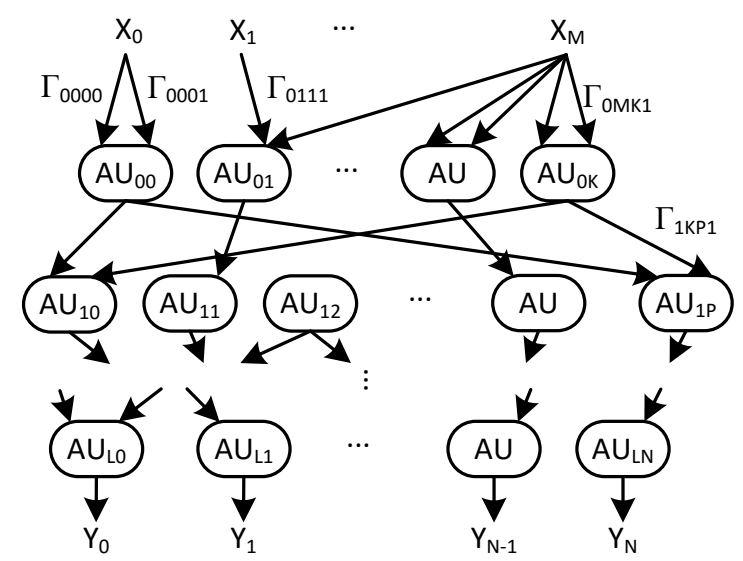

Figure 10.8: General datapath structure.

we can identify the following operations:
OP1: $t 1=a+b$
OP2: $t 2=c+d$
OP3: $y=t 1+t 2$
OP4: $z=t 1-t 2$

Given an initial mapping of all instructions, the optimization process is then used to find better mappings.

Given these model variables, we may use many different optimization goals, which are easily setup based on the path variables $\Gamma_{l s d p}$. Some possible examples include:

- Number of paths: The number of paths will be directly proportional to the number of physical wires that must be routed between execution units, thus a low number of preferable.

- Minimize (maximum) fan-in: High fan-in to an execution unit input port means that this port requires a larger and potentially slower multiplexer. We'd like to keep this as low as possible.

- Minimize (maximum) fan-out: High fan-out is equivalent to that an execution unit has many successors. A higher fan-out means a 
higher electrical load, which slows down the circuitry and possibly increases energy consumption.

- Avoid expensive paths: If we consider a target physical layout or placement of arithmetic units, one may penalize physically long paths or other paths that present a higher load, or paths that complicate routing.

One, several or all of these objectives can be weighted to form an objective function, such as:

$$
\text { minimize } \sum_{\forall l, \forall s, \forall d, \forall p}\left(\alpha_{l s d p} \Gamma_{l s d p}\right)+\beta \mu+\gamma \nu
$$

The first part represents the number of paths, possibly with individual weighting factors $\alpha_{l s d p}$ to model different costs for different paths. The variables $\mu$ and $\nu$ represent the maximum fan-in and maximum fan-out respectively, each individually weighted with $\beta$ and $\gamma$. These may be generated by the following constraints:

$$
\begin{gathered}
\text { Fan-in: } \forall l, \forall d, \forall p: \sum_{\forall s} \Gamma_{l s d p} \leq \mu \\
\text { Fan-out: } \forall l, \forall s: \sum_{\forall d, \forall p} \Gamma_{l s d p} \leq \nu
\end{gathered}
$$

To complete the model, we now need to set constraints on the paths and operation mapping variables.

\subsubsection{General constraints for operation mapping}

We start by introducing general constraints for operation mapping. Each arithmetic unit can, for any instruction, maximally be allocated one operation. This may be formulated as:

$$
\forall i, \forall l, \forall u: \sum_{\forall o} \mathcal{O}_{i l u o} \leq 1
$$

Although the internal connections of the datapath are arbitrary, the inputs and outputs to the datapath should be ordered to implement the 
correct function. The simplest way of modelling ordered inputs is to introduce a virtual layer of arithmetic units, layer 0 , with fixed mappings. The constraint may be formulated as,

$$
\forall i, l=0, \forall u, \forall o: \text { if } u==o \text { then } \mathcal{O}_{\text {iluo }}=1 \text { else } \mathcal{O}_{\text {iluo }}=0,
$$

which means that virtual arithmetic unit $u$ is implementing virtual operation $o$. Ordered outputs can be implemented on the final layer, layer $\mathrm{L}$, using the following constraints:

$$
\forall i, l=L, \forall u, \forall o: \text { if } u \neq o \text { then } \mathcal{O}_{\text {iluo }}=0 .
$$

Note that we may fix the location of all potential source operands, although all of them are possibly not used by an instruction. However, we do not set any value for $\mathcal{O}_{\text {iluo }}$ when $u=o$ and $l=L$, since all instructions may not use all datapath outputs. We simply make sure that an output will be mapped correctly, if it exists.

\subsubsection{Mapping instructions to the datapath}

To map an instruction to the datapath, we need to map all of its individual operations and then generate constraints that produce the necessary interconnect paths. Given that an operation $o$ of instruction $i$ should be mapped to one of the arithmetic units in layer $l$, we can start by adding the constraint

$$
\sum_{\forall u} \mathcal{O}_{i l u o}=1
$$

which ensures that the operation is mapped to exactly one of the arithmetic units in layer $l$. If a particular operation $\mathcal{O}_{0}$ has predecessor operation(s) $\operatorname{pred}\left(\mathcal{O}_{0}\right)$ in the previous arithmetic unit layer, we must add appropriate constraints to add interconnect paths. Since the predecessor operation(s) pred $\left(\mathcal{O}_{0}\right)$ may be mapped to arbitrary arithmetic units in a previous layer (input operands excluded), we need to generate constraints for all possible mappings of $\mathcal{O}_{0}$ and $\operatorname{pred}\left(\mathcal{O}_{0}\right)$. Also, since $\mathcal{O}_{0}$ may be a commutative operation, the predecessors can be mapped to the input ports of an arithmetic unit in any order. 
Given that an operation may have one or two predecessor operations, and that the order of the inputs may be arbitrary, we can identify a few potential cases:

- Single predecessor, fixed port: An operation has a single predecessor operation, whose result must be mapped to a fixed input port, either input port 0 or port 1.

- Single predecessor, arbitrary port: An operation has a single predecessor operation and an arithmetic unit can perform the operation on any input port.

- Dual predecessors, non-commutative: An operation has two predecessors, the operation is non-commutative and the arithmetic unit does not support both $a \oplus b$ and $b \oplus a$. Mapping must be restricted to the correct ports.

- Dual predecessors, commutative: An operation has two predecessor operations, the operation is commutative or the arithmetic unit supports commutative execution of the operation. The order is unimportant.

These four cases results in different constraints to be set when mapping an operation.

Starting from the simplest case, which is an operation with a single predecessor that should be mapped to a fixed port $p$, we can implement constraints as follows: If operation $o$ is mapped to AU $u$ in layer $l$ and the predecessor operation $o_{p}$ is mapped to $\mathrm{AU} u_{p}$ in layer $(l-1)$, the following constraints will add the appropriate interconnect path:

$$
\forall \text { pairs }\left\{u, u_{p}\right\}: \Gamma_{l u_{p} u p} \geq \mathcal{O}_{i l u o}+\mathcal{O}_{i(l-1) u_{p} o_{p}}-1 \text {. }
$$

The constraint must be generated for all pairs of $\left\{u, u_{p}\right\}$, since the operations can be mapped arbitrarily in general. This constraint is easily extended into the arbitrary port case, by instead generating

$$
\forall \text { pairs }\left\{u, u_{p}\right\}: \Gamma_{l u_{p} u 0}+\Gamma_{l u_{p} u 1} \geq \mathcal{O}_{\text {iluo }}+\mathcal{O}_{i(l-1) u_{p} o_{p}}-1
$$




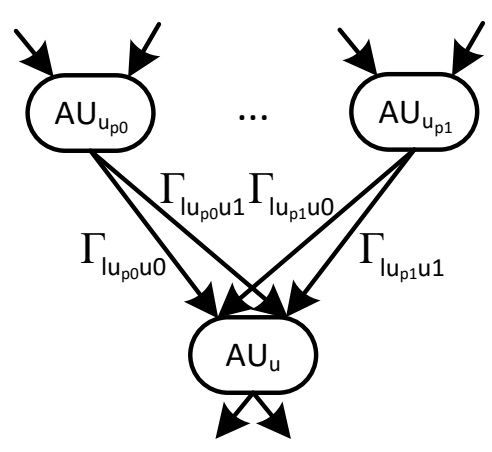

Figure 10.9: Mapping binary commutative operations to the datapath.

which ensures that a path exists to at least one of either input port 0 or 1 .

Binary operations require additional attention. The unary case constraints for the fixed-port case and commutative case respectively, are still valid for binary operations, if they are introduced for the two predecessor operations separately. If operation $o$ is mapped to AU $u$ in layer $l$ and the predecessor operations $o_{p 0}$ and $o_{p 1}$ are mapped to AUs $u_{p 0}$ and $u_{p 1}$ respectively in layer $(l-1)$, the fixed-port constraints are

$$
\begin{aligned}
& \forall \text { pairs }\left\{u, u_{p 0}\right\}: \Gamma_{l u_{p 0} u 0} \geq \mathcal{O}_{i l u o}+\mathcal{O}_{i(l-1) u_{p 0} o_{p 0}}-1 \\
& \forall \text { pairs }\left\{u, u_{p 1}\right\}: \Gamma_{l u_{p 1} u 1} \geq \mathcal{O}_{i l u o}+\mathcal{O}_{i(l-1) u_{p 1} o_{p 1}}-1
\end{aligned}
$$

and in the commutative case

$$
\begin{aligned}
& \forall \text { pairs }\left\{u, u_{p 0}\right\}: \Gamma_{l u_{p 0} u 0}+\Gamma_{l u_{p 0} u 1} \geq \mathcal{O}_{i l u o}+\mathcal{O}_{i(l-1) u_{p 0} o_{p 0}}-1 \\
& \forall \text { pairs }\left\{u, u_{p 1}\right\}: \Gamma_{l u_{p 1} u 0}+\Gamma_{l u_{p 1} u 1} \geq \mathcal{O}_{i l u o}+\mathcal{O}_{i(l-1) u_{p 1} o_{p 1}}-1 .
\end{aligned}
$$

However, in the commutative binary case, this is not enough. Specifically, these constraints do not prohibit that the results from both predecessors are mapped to the same input port.

If the current operation and its predecessors are mapped to some given arithmetic units, there are four potential paths that must be considered, as shown in Figure 10.9. The constraint from Equation 10.12 makes sure that either path $\Gamma_{l u_{p 0} u 0}$ or path $\Gamma_{l u_{p 0} u 1}$ exist and likewise for 
Equation 10.13 and paths $\Gamma_{l u_{p 1} u 0}$ and $\Gamma_{l u_{p 1} u 1}$. To make sure that both predecessors are not mapped to the same input port, we add the following help variable and constraints:

$$
\begin{aligned}
& x_{u u_{p 0} u_{p 1}}=\mathcal{O}_{i l u o}+\mathcal{O}_{i(l-1) u_{p 0} o_{p 0}}+\mathcal{O}_{i(l-1) u_{p 1} o_{p 1}}-2 \\
& \forall \text { triplets }\left\{u, u_{p 0}, u_{p 1}\right\}: \Gamma_{l u_{p 0} u 0}+\Gamma_{l u_{p 1} u 0} \geq x_{u u_{p 0} u_{p 1}} \\
& \forall \text { triplets }\left\{u, u_{p 0}, u_{p 1}\right\}: \Gamma_{l u_{p 0} u 1}+\Gamma_{l u_{p 1} u 1} \geq x_{u u_{p 0} u_{p 1}}
\end{aligned}
$$

The help variable $x_{u u_{p 0} u_{p 1}}$ in Equation 10.14 is equal to 1, if the three operations are mapped to arithmetic units $u, u_{p 0}$ and $u_{p 1}$ respectively. Otherwise $x_{u u_{p 0} u_{p 1}}<1$. If $x_{u u_{p 0} u_{p 1}}=1$, at least one of $\Gamma_{l u_{p 0} u 0}$ or $\Gamma_{l u_{p 1} u 0}$ is equal to 1 according to Equation 10.15. As an example, if $\Gamma_{l u_{p 0} u 0}=0$ then $\Gamma_{l u_{p 1} u 0}=1$. Additionally, since $\Gamma_{l u_{p 0} u 0}=0$, then $\Gamma_{l u_{p 0} u 1}=1$ according to Equation 10.12. Since $\Gamma_{l u_{p 1} u 0}=1$ and $\Gamma_{l u_{p 0} u 1}=1$ this means that predecessor 1 has been mapped to port 0 and predecessor 0 has been mapped to port 1 , which is a valid mapping. Similar arguments can made if any other path variable is zero and only valid mappings will be generated. Note that nothing prevents all path variables to be 1 , which may happen when we map multiple instructions to the datapath.

We have not discussed the case when a single predecessor is routed to both inputs of a receiving binary operation. This can easily be handled as well. However, in practice it may be better to route the predecessor to only one of the two input ports and duplicate the value inside the receiving arithmetic unit.

\subsubsection{Optional constraints}

There are many additional constraints that can be added to the model if desired. Any of these optional constraints must be added with care, as adding too tight constraints may result in a model with no feasible solutions. Instead of penalizing fan-in, fan-out and expensive paths in the objective function, one can forbid some mappings completely. A largest allowed fan-in can be set as

$$
\forall l, \forall d, \forall p: \sum_{\forall s} \Gamma_{l s d p} \leq M F I(l, d, p)
$$


where $\operatorname{MFI}(l, d, p)$ is the desired maximum fan-in for a particular input port of an arithmetic block in layer $l$. Similarly, the maximum fan-out of an arithmetic unit output can be bounded by setting

$$
\forall l, \forall s: \sum_{\forall d, \forall p} \Gamma_{l s d p} \leq M F O(l, s)
$$

Any undesired path in the interconnect network can be disallowed by simply setting

$$
\forall \text { PROHIBITED PATHS: } \Gamma_{l s d p}=0
$$

In addition to path constraints, one can add operation mapping constraints. This is suitable to do if all arithmetic units are not equal and some AUs simply cannot perform certain operations. This is easily added as:

$$
\forall \text { PROHIBITED OPERATION MAPPINGS: } \mathcal{O}_{\text {iluo }}=0
$$

\subsubsection{Limitations}

The proposed optimization model is built from a pre-existing mapping of an instruction, which defines all operations and at which pipeline layer each operation should be performed. However, in some cases it may be possible to move operations between layers for better results. It may also be possible to change which operations to perform. As an example, an instruction $z=(a+b)+(c+d)$ could be changed into e.g. $z=(a+c)+(b+$ $d)$, which algebraically gives the same results. None of these possibilities are covered by the current optimization model. One may consider this limitation when doing the initial mapping for the instructions that may be affected.

\subsubsection{Evaluation}

The proposed optimization model has been evaluated on the example datapath structure from Figure 10.4, by targeting the arithmetic unit network of 3 layers with 8 arithmetic units per layer. A set of 155 instructions with 31 unique network configurations was used for the evalua- 
tion. Note that many actual instructions use the same network configuration and only differ in the arithmetic operation performed at the arithmetic units. The instructions mapped to the datapath include general and application-specific instructions for multiple applications, including simple SIMD instructions, real-valued and complex-valued reductions, 2, 3, 4 and 5-point butterfly instructions, MPEG/JPEG/H.264 DCT and IDCT acceleration instructions, filter-accelerating instructions, multiple sorting networks, median calculation instructions and prefix operations.

The original mapping of the instructions have been made by hand in the order that they were originally implemented during the research process. As each new instruction was mapped, some level of effort was spent to avoid adding many new paths compared to the already existing instructions. This is a very tedious process with sub-optimal results. If 8 operations should be mapped to a given layer in the datapath, this can be done in $8 !=40320$ ways and the mapping over all layers in $(8 !)^{(3-1)} \approx 1.6 \cdot 10^{9}$ ways, since there are 3 layers. Note that the final layer can only be mapped in one way, as the order of the datapath outputs would change otherwise. Now, considering the possible combinations of 31 different instructions, we end up with a total of $(8 !)^{(3-1) \cdot 31} \approx 3.5 \cdot 10^{285}$ possible ways to map the operations. In practice, all instructions do not map 8 operations per layer. The actual number of possibilities for the set of test instructions is $1.9 \cdot 10^{237}$, which is still huge value. Additionally, for any mapping of all operations and instructions, one may also choose which signal is routed to which input port, at least for commutative operations. The original hand-made implementation introduces 153 paths in the network.

The datapath was optimized using the Gurobi ILP optimizer [106]. Figure 10.10 shows the best result in number of paths found over optimization time when optimizing for a minimal number of paths. The lower bound of the LP-relaxed model is also reported. The solver finds a solution of equal cost compared to the hand-made implementation in roughly 35 seconds. After 2600 seconds, a solution with 115 paths is found. The solver was run for a total of 50000 seconds, but no better so- 


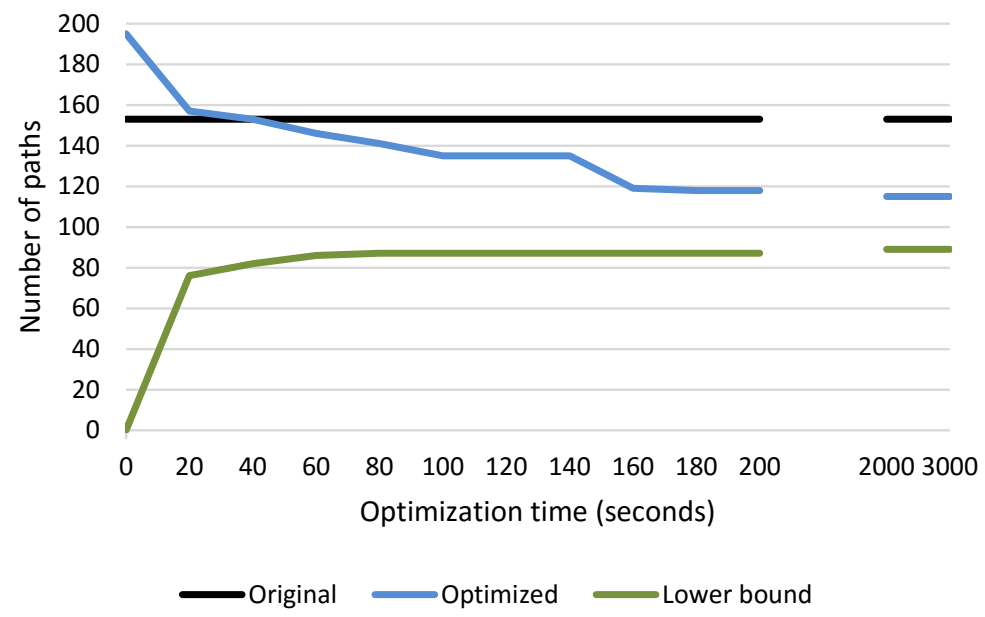

Figure 10.10: Optimization result over run-time.

lution was found. At this time, the lower bound was 89 paths. Although an optimal solution was not found in reasonable time, the number of paths was reduced by $25 \%$.

It is of interest to see what an iterative instruction mapping could achieve. This was investigated by using the ILP model and adding only one instruction at a time. The solver was allowed to run until the optimum was found, after which the operation mapping of that instruction was fixed and the model was resolved with a newly added instruction. The result will depend on what order the instructions are added, but also what actual optimum is chosen at each stage, since there may be multiple choices of equal cost. The instructions were sorted according to how many paths they would individually require. Instructions of equal complexity were added in random order. Two alternative instruction orderings were tested: sorted from largest to smallest (LF, largest-first) and smallest to largest (SF, smallest-first). This resulted in 124 and 133 paths respectively after optimization. 
Table 10.1: Relative hardware cost and energy consumption for the arithmetic unit network and complete datapath before and after optimization.

\begin{tabular}{l|c|cc|cc}
\hline & & \multicolumn{2}{|c|}{ Relative area [\%] } & \multicolumn{2}{c}{ Relative energy [\%] } \\
Datapath ver. & \#Links & AU-net & Datapath & AU-net & Datapath \\
\hline Original & 153 & $100 \%$ & $100 \%$ & $100 \%$ & $100 \%$ \\
Iterative, SF & 133 & $86 \%$ & $95 \%$ & $87 \%$ & $95 \%$ \\
Iterative, LF & 124 & $81 \%$ & $93 \%$ & $87 \%$ & $90 \%$ \\
Global search & 115 & $76 \%$ & $91 \%$ & $72 \%$ & $85 \%$ \\
\hline \hline
\end{tabular}

In order to estimate the savings in actual hardware cost and energy consumption, the four different datapaths were synthesized with a $28 \mathrm{~nm}$ FD-SOI technology library. Energy consumption was estimated by running 10 typical kernels, e.g. FIR-filtering, FFT and matrix multiplication. The kernels were simulated at the gate-level with switching activity and the average energy consumption was calculated. The results are shown in Table 10.1. Values are reported both for the AU-network in isolation and for the complete datapath. The fully optimized model resulted in a $24 \%$ reduction of area and $28 \%$ reduction in energy consumption in the AU-network, compared to the original datapath. This resulted in an overall reduction of $9 \%$ area and 15\% energy for the whole datapath.

\subsubsection{Summary}

The suggested datapath optimization model can automate instruction mapping and deliver significant savings in area and energy, both for processor datapath structures, but also other similar pipelined structures in custom processing elements. If a datapath design is generated by selecting instructions for a database of pre-defined instructions, which is then enhanced with additional custom application-specific instructions, an optimized datapath can be generated within minutes or hours, depending on datapath complexity and how much effort one wants to spend on the optimization process. It is a valuable tool in a platform generation toolbox and can eliminate considerable manual labor. 


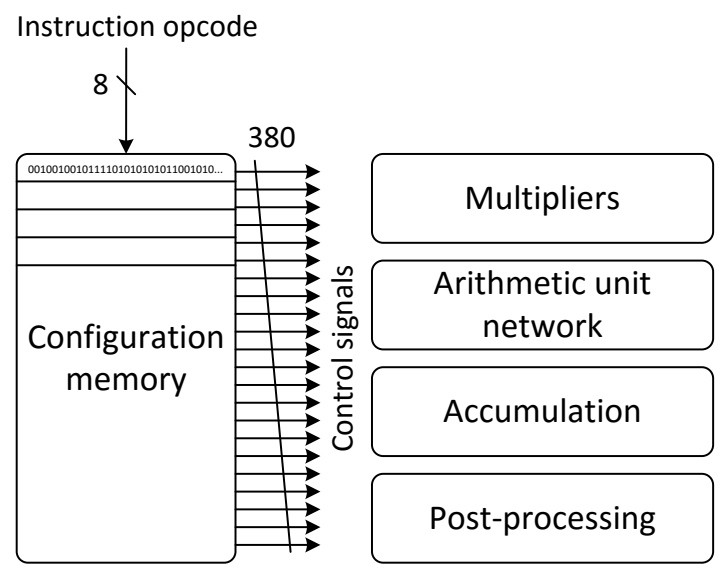

Figure 10.11: A reconfigurable datapath structure.

\subsection{Datapath reconfigurability}

Reconfigurability is a property of a system, which allows changes in behavior by loading different configurations [107,108], such as a processor instruction set. It can be applied at many different levels, from gate-level manipulations, to the interconnect network between functional units or beyond. This may make it possible to push some design decisions from platform design-time until run-time, improve flexibility or to create new functionality based on updated application standards.

One possible way to make a processor datapath reconfigurable is to expose all datapath control signals to a configuration memory, as shown in Figure 10.11. A datapath opcode is given as an input to the configuration memory and the control signals for that particular instruction is then fetched from the memory. The configuration memory can be programmed at e.g. system startup time, or before any kernel execution. It allows different instruction sets to be loaded depending on application needs.

Reconfigurability introduces flexibility, but has a significant cost. First of all, the decoding logic for the datapath control is replaced by a much more expensive configuration memory. Secondly, there are less oppor- 
Table 10.2: Hardware cost and power consumption for fixed-function versus reconfigurable datapath. The optimized version of the fixed-function datapath is also included.

\begin{tabular}{l|cc|cc|cc}
\hline \hline & \multicolumn{5}{|c}{ Relative hardware area/power [\%] } \\
Unit & Fixed function & Optimized & Reconfigurable \\
& Area & Power & Area & Power & Area & Power \\
\hline MUL stage & $30 \%$ & $17 \%$ & $30 \%$ & $15 \%$ & $30 \%$ & $18 \%$ \\
AU stage & $31 \%$ & $28 \%$ & $24 \%$ & $20 \%$ & $58 \%$ & $71 \%$ \\
ACC stage & $33 \%$ & $36 \%$ & $30 \%$ & $32 \%$ & $33 \%$ & $39 \%$ \\
Control signals & $1 \%$ & $1 \%$ & $1 \%$ & $1 \%$ & $132 \%$ & $15 \%$ \\
Other & $4 \%$ & $17 \%$ & $4 \%$ & $17 \%$ & $4 \%$ & $21 \%$ \\
\hline Total & $\mathbf{1 0 0} \%$ & $\mathbf{1 0 0} \%$ & $\mathbf{9 0} \%$ & $\mathbf{8 5} \%$ & $\mathbf{2 5 8} \%$ & $\mathbf{1 6 5} \%$ \\
\hline \hline
\end{tabular}

tunities for synthesis optimizations if the datapath function is not fixed. In addition to an increased hardware cost, there are several other potential drawbacks such as increased energy consumption and increased signal propagation delay which could limit clock frequency. There are also effects on software, since the datapath configuration must be loaded at run-time, costing additional energy and execution time. To motivate a reconfigurable datapath the benefits must outweigh these drawbacks.

The cost of implementing the reconfigurable datapath from Figure 10.11 has been compared to a fixed-function datapath. A datapath opcode size of 8 bits was used, which makes it possible to store 256 different instructions. There are a total of 380 control signal bits to our datapath, which makes the size of configuration memory $256 \cdot 380=97280$ bits.

The relative cost and power consumption of a fixed-function and a reconfigurable datapath is shown in Table 10.2. The datapath named Fixed-function is the original datapath with hand-mapped instructions. The datapath version named Optimized is the datapath which has been optimized using the ILP model from Section 10.4. The power consumption was once again extracted as an average from gate-level simulations of 10 standard kernels. We can clearly see that reconfiguration does not 
come for free. The configuration memory has a very high hardware cost, but the other datapath components increase in area as well, especially the AU network. This is because in the fixed-function datapath, many interconnect paths and other functions will be removed because they are not used. However, for the reconfigurable datapath, all paths will be kept, which ensures that we can implement arbitrary interconnections. The energy overhead is not as severe as the area overhead, but is still very high. One important reason why the energy consumed by control signals is not too high is because control signal lookup is significantly reduced by multi-cycle HOF execution. It is certainly possible to decrease the overhead of the reconfigurable datapath by several means. One possibility is to implement all instructions of the fixed-function datapath as fixed instructions in the reconfigurable datapath, and only provide a very small memory for a few programmable instructions. This would reduce the cost of the configuration memory, but not the datapath logic overhead. Another possibility is to reduce the possible paths in the arithmetic unit network, but this restricts what new instruction that can be added. Still, the overhead of making the datapath reconfigurable may still be high, and it may not be worth its cost.

\subsection{Summary}

This chapter discussed design considerations regarding the implementation of processor datapaths. An important design consideration in the context of a configurable platform is to be able to keep compatibility with an existing kernel library. Additional application-specific instructions may then be added to the datapath structure. An optimization model was presented, which can be used to reduce datapath interconnect and improve energy efficiency, once an appropriate instruction set has been selected. Finally, the cost of a reconfigurable datapath was investigated. Although a reconfigurable datapath may have some benefits, the reduced energy efficiency and increased hardware cost may make it less useful in practice. 


\section{Chapter 11 \\ The ePUMA Matrix Processing Element}

The previous chapters have presented the HOF instruction concept and proposed suitable memory subsystems for such an architecture. Additionally, the design and optimization of complex datapath structures have been discussed. To be able to evaluate these proposals and collect some real-world system data, the ePUMA Matrix Processing Element (MPE) will now be introduced. The MPE is a practical example of a HOF-based architecture that is used as a compute-optimized DSP within an ePUMA compute cluster. A large library of DSP kernel routines have been implemented and will be used to evaluate the hardware concepts in relation to other contemporary architectures. Although this is only a single instance of a HOF-based architecture, it will still be useful to estimate trends for other similar architectures. In addition, other important architecture features will be discussed, and some important consequences of using a HOF-based architecture will be outlined.

\subsection{Goals}

Although the MPE can be considered an application-specific processor after it has been configured with application-specific instructions, one of 
the main design goals is to exploit the similarities between relevant application functions to enable maximum reutilization of the basic architecture. In order to design an appropriate demonstration platform, the main MPE configuration that will be used for evaluation has been selected in such a way that it represents a reasonable trade-off between the requirements of the applications highlighted in Chapter 3. The possibly most important design parameter is the size of the memories, as memories will typically dominate total implementation cost. In order to support a wide range of compute kernels, memory sizes must be selected so that the relevant kernels can be implemented on an isolated MPE. Algorithms that must be executed over multiple MPEs or even multiple compute clusters involve many other system aspects, which are not directly relevant to the evaluation of the core-level concepts.

The overall main goal of the MPE is to demonstrate the benefits of HOF computing and the proposed memory subsystem. In order to make a fair comparison with other architectures, the MPE is made similar in capability to the most relevant competitors. Since most of the data available for comparison is based on general-purpose DSPs, we will primarily use a general MPE configuration. This means that the architecture is equipped with a complete set of general- and application-specific instructions for multiple applications, and support for a wide range of standard data types.

\subsection{The MPE architecture}

The MPE architecture has been designed to act as a tightly coupled accelerator in an ePUMA compute cluster. The cluster's main processor, the compute cluster controller (CCC), executes the main execution thread in a cluster. It may call compute kernels, which off-loads work to one of possibly multiple local MPEs. The CCC assigns a set of LVM memories to the MPE, which stores the input-, output- and intermediate data. The MPE will then execute its compute kernel independent of the CCC until the kernel terminates. 


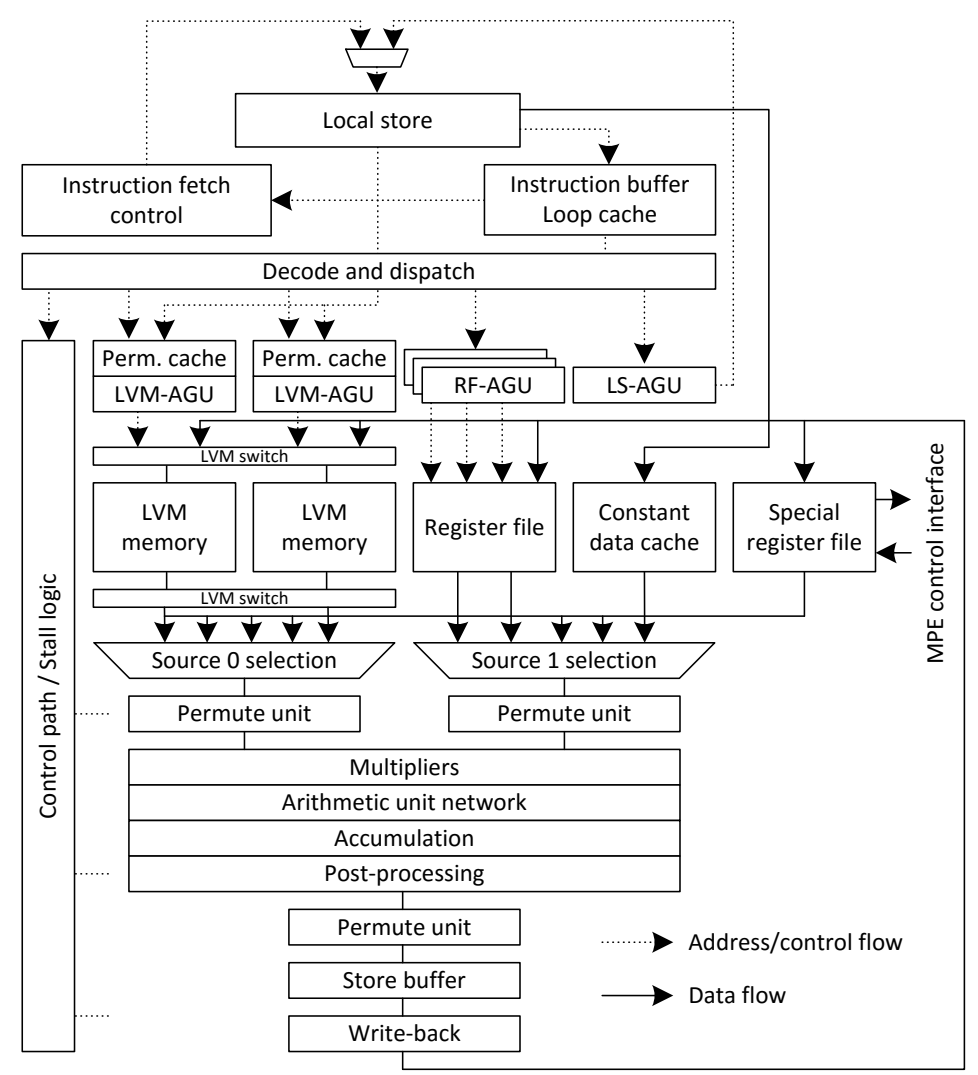

Figure 11.1: Overview of the MPE architecture.

Figure 11.1 depicts the main components of an MPE. It includes the already mentioned LVM memories and their related permutation vector caches, a cache for constant data, a single local store for instructions, permutation tables and constants, and the example datapath that was introduced in Figure 10.4. Three permutation units are available for individual duplication and permutation of operands for the two source operands and the destination operand respectively. In addition, it contains a general register file for general compute data and memory addresses, and a special register file which contains parameter registers, 
Table 11.1: ePUMA MPE specification.

\begin{tabular}{l|l}
\hline \hline Property & Value \\
\hline Natural processing width & 128 -bits \\
Instruction width & $32 / 64 / 128$ bits \\
\hline Num LVMs & 3 \\
LVM size & $64 \mathrm{kB}$ \\
LVM banks/mem & 8 \\
LVM bank width & 16 bits \\
\hline Local store size & $16 \mathrm{kB}$ \\
Local store width & 128 bits (single bank) \\
Register file size & $128 \mathrm{~B}$ \\
Special registers & $256 \mathrm{~B}$ \\
Permutation cache size & $128 \mathrm{~B}$ \\
Constants cache size & $16 \mathrm{~B}$ \\
\hline Datapath latency & $1 / 4$ cycles (typical) \\
Data types & $8-, 16-$, 32-bit real fixed-point \\
& $16-, 32-$ bit complex fixed-point \\
& 32 -bit real/complex floating-point \\
\hline \hline
\end{tabular}

address registers, status registers and other control registers. The special register file is visible to the CCC and is used to control the MPE.

The default configuration for MPE evaluation is summarized in Table 11.1. The natural processing width of 128-bits and LVM memories with 8 16-bit memory banks per memory have been selected as a good trade-off between processing performance and area. The natural processing width of SIMD architectures vary greatly from architecture to architecture, and some even make it variable for increased flexibility [61, 109, 110]. For evaluation purposes, 128 bits is a reasonable trade-off that works well for many situations. Also, the hardware complexity of the permutation units and the datapath grows more than linearly, so a fixed not too wide processing width makes sense.

A HOF instruction may require many instruction word bits to encode 


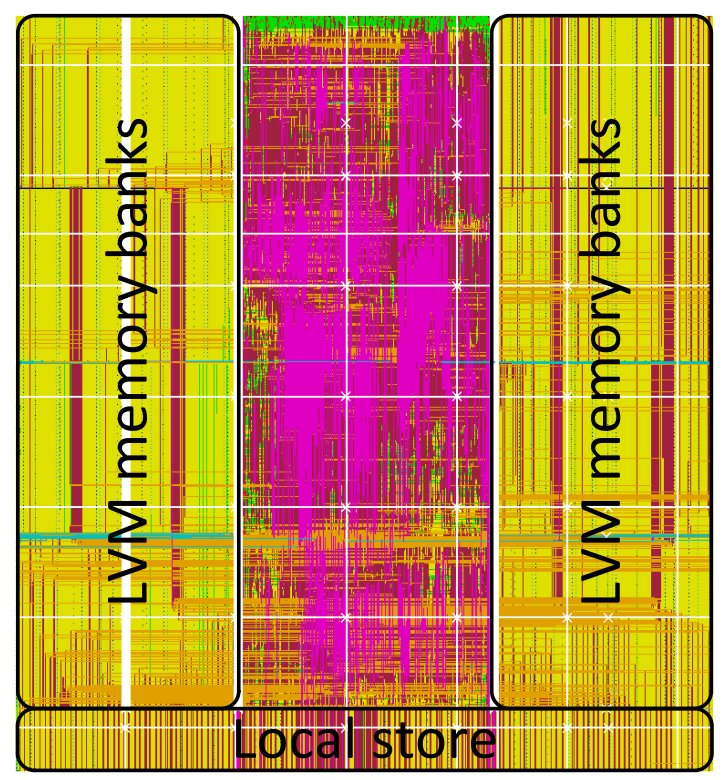

Figure 11.2: The VLSI layout of an MPE.

all control and address information. On the other hand, such an instruction may need support from multiple configuration instructions before execution, operations which may be rather simple. A variable length instruction format has therefore been chosen, so that common control operations can be encoded with fewer bits. Although a fixed-length format may be simpler to implement, the potential code size savings are considerable. Furthermore, instruction fetching requirements are reduced, which reduces the load on the local store. The maximum size of 128 bits for an instruction is still smaller than the instruction word in many typical VLIW DSPs [58].

\subsection{Physical characteristics}

Several physical layouts of MPEs have been implemented for evaluation purposes. One such layout is shown in Figure 11.2, which includes the 
Table 11.2: Hardware characteristics of an MPE including 2 LVM memories.

\begin{tabular}{l|l}
\hline \hline Property & Value \\
\hline Design frequency & $500 \mathrm{MHz}$ \\
Target tech. & 28nm FD-SOI \\
Logic area & $0.14 \mathrm{~mm}^{2}$ \\
Memory area & $0.29 \mathrm{~mm}^{2}$ \\
Total area & $0.43 \mathrm{~mm}^{2}$ \\
Logic power & $20 \mathrm{~mW}$ \\
Memory power & $50 \mathrm{~mW}$ \\
Total power & $70 \mathrm{~mW}$ \\
\hline \hline
\end{tabular}

processor core, a local store and two tightly coupled LVM memories. In a full system the LVM memories are a shared resource within a compute cluster and their placement relative to the core is likely different, which will impact signal timing and energy efficiency. At the same time, the LVMs should not be ignored. Any MPE will require some memories for compute data. Time-sharing of the memories between cores is unfeasible, primarily since many practical kernels will approach $100 \%$ time utilization of the memories. It is therefore reasonable to include the cost of LVM memories in total MPE cost. The MPE layout is based on a design frequency of 500MHZ and is implemented in 28nm FD-SOI technology.

Table 11.2 lists MPE area and power consumption. Of the total area, $68 \%$ is used for memories, which leaves $32 \%$ for the core logic. The largest logic component is the datapath, which consumes $57 \%$ of the logic area or $18 \%$ of the total area (memory+logic). This is also the critical part in terms of timing. The remaining $43 \%$ of the core area (13\% of total) is shared among all other components such as register files, permutation caches, the instruction decoder and the control path. In some sense these components can be considered fixed and should be available on any MPE instance for compatibility reasons. However, this part is rather small and a platform designer can greatly affect the total hardware cost by simply changing the size of the memories and the datapath instruction set. 


\subsection{Pipeline scheduling}

An important design decision is the visibility of the processor's pipeline. Simpler processors may use a statically scheduled pipeline, which means that the program code must take the pipeline into account to produce correct behavior. For instance, a data dependency between two instructions must be handled explicitly by inserting unrelated instructions in between. If no unrelated instructions are available, NOP-instructions must be inserted. In contrast, a dynamically scheduled pipeline handles data hazards and structural hazards automatically using stall logic.

A dynamically scheduled pipeline has many advantages and this has been chosen for the MPE. First of all, a HOF instruction reading and writing to the same LVM memory under many clock cycles is hard to handle statically with a single-ported memory. Execution would have to be paused at regular intervals to create time slots for memory writeback. Additionally it may become impossible to execute an assembly kernel on different MPE configurations if they are organized differently. By using a dynamically scheduled pipeline we also introduce the possibility for some features which may make it impossible to statically determine whether or not a data hazard exists. One such feature is register file addressing, which will be described in Section 11.7. The main drawbacks of the dynamic pipeline is increased hardware cost and an increase hardware verification effort. Energy consumption may increase, but could also decrease since we do not have to fetch and decode NOP instructions.

\subsection{Memory consistency}

The MPE implements a simplified form of stall logic for LVM memories to guarantee that any memory read will return the most recently written value to that location. The example MPE configuration has LVM memories with 8 memory banks each. This means that up to 8 memory addresses may be generated per clock cycle and memory. Tracking reads and writes to individual memory locations may come with a great cost. 
Additionally, since memory addresses are generated dynamically by the AGUs, it could be that one instruction reads some memory location before the write addresses of an earlier instruction are not even yet generated. Also, since long HOF instructions are executed non-atomically (over multiple cycles), potential self-dependencies between the source and destination operands may introduce race conditions.

The MPE deals with the above mentioned problems as follows: Selfdependencies of a HOF instruction must be guaranteed to be safe by the programmer or tool-chain. A fixed latency between reads and writes should not be assumed, since this could be different for different MPE instances. If dependencies are present, a HOF instruction should be split into multiple instructions. This behavior is motivated by the fact that any HOF instruction which uses the same memory for both source and destination operands would see a large reduction of performance otherwise. The hardware guarantees sequential memory consistency between different instructions by making sure that all memory writes of one instruction are completed before a subsequent instruction may read the same memory [111]. This may seem like a performance issue, but most kernels use HOF instructions for streaming computations over many cycles. When a HOF instruction terminates, it is simplest to wait for all memory writes to be completed before a subsequent instruction can read the newly produced data, not because of data dependencies but rather because the memory is single-ported and will be busy the same total number of cycles regardless of memory operation ordering.

Some kernels may be negatively affected by following sequential consistency strictly. The MPE solves this by making it possible to disable the memory stall logic. In this case, memory barrier instructions must be inserted to guarantee correct operation. The memory consistency mode can be selected when a kernel starts, or during kernel execution by modifying a control register. This feature will be evaluated in Chapter 15. 


\subsection{Reducing performance unpredictability}

An important design goal of the MPE is to be able to eliminate most sources of performance unpredictability, which eases application performance analysis. This may be of huge practical importance when analyzing real-time constraints. Because the LVM memories are local and exclusive to the core during processing, and the execution time of HOF instructions are predictable, many MPE compute kernels are guaranteed to be executed with a fixed execution time. Many kernels accept the number of input elements as a kernel input parameter and the actual execution time will naturally depend on it, but once it is known, the execution time can easily be determined. Unpredictability is easily introduced into any kernel by inserting some data dependent control flow. In practice, however, performance unpredictability in DSP kernels can often be avoided with proper hardware support. This section will briefly survey the features implemented in the MPE.

\subsubsection{Vector length independent execution and iteration capability}

The HOF concept naturally handles vectors of arbitrary length and the vector size can even be passed in a register. In contrast, SIMD processors operate on vector registers with fixed width and multiple instructions is required to handle vectors of other lengths. For maximum performance of arbitrary vector lengths, one may have to provide different implementations with various degrees of loop unrolling and other optimizations. Together with other uncertainties such as branch prediction and cache misses, the execution time cannot be determined exactly.

In addition to the two levels of iteration control provided by a HOF instruction, the MPE supports an additional level of iteration: hardware loops. It allows a group of instructions to be repeated multiple times, where the iteration value may be instruction-carried or register-carried. The use of hardware loops makes it easy to predict instruction flow without the use of e.g. branch prediction. 
Listing 11.1: Absolute value implementation using an if-else-statement.

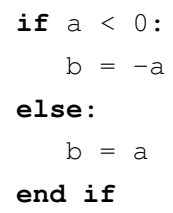

\subsubsection{Control flow removal}

Some unpredictability can be avoided with proper instruction set support. Consider for example the computation of elementwise absolute value of an input vector $b=a b s(a)$, without an absolute value instruction. It may be implemented as shown in Listing 11.1. Depending on the outcome of the conditional check, the execution time may vary. To make matters worse, if positive and negative values are roughly equally common and randomly distributed, a branch predictor will make many mispredictions. An absolute value instruction solves all of these issues. During the implementation of the MPE kernel set, the potential for moving control flow from the instruction flow to the datapath has been investigated when applicable. This has resulted in many instructions, including absolute value, saturation, thresholding, clipping, add-compareselect and other instructions.

\subsubsection{Predicated execution and control flow}

Predicated instruction execution is the conditional execution of an instruction [107]. It may sometimes be used to replace more expensive conditional branch instructions. As an example, an if-else-statement can be translated into machine code that first evaluates the condition and then executes both the if-branch and the else-branch. The instructions in the if-branch and else-branch respectively, will only take effect conditionally based on the outcome of the condition evaluation. To determine if an instruction should be executed or not, each instruction must be tagged with a condition code, which consumes additional instruction 
word space. Since all instructions are executed, the performance can be predictable.

For branches that are easily predicted, a branch predictor works well and the condition code bits in the instruction word simply consume unnecessary space, which may be better used for other purposes, such as increasing the number of architectural registers [112]. For hard-to-predict branches, predicated execution may be a better choice [113]. In [114] the authors propose a scheme where the hardware can switch between branch prediction and predication dynamically based on a run-time confidence estimation.

The MPE implements predication as a vector mask, similarly to the implementation in CRAY-1 [115]. For example, a vector compare instruction can be used to generate a vector of comparison results. These results can then be used as a mask for a subsequent vector operation. For instructions based on the map pattern, predication will be used as a write-mask. For reduce and map-reduce instructions, predication will instead be used as a read mask, which makes it possible to exclude some elements from the reduction based on a run-time decision.

Most of the kernels in the MPE kernel library do not contain jump instructions. The two levels of iteration provided by HOF instructions and one level of hardware iteration is enough to implement most kernels. The MPE is not designed to handle application control flow, which instead is performed by the CCC. Because of this, the MPE does not implement branch prediction. However, the MPE allows any instruction to enable or disable condition flag generation. By doing so, it is possible to separate the instruction used to set the processor's condition flags from the instruction that uses the flags, to hide flag generation latency.

\subsection{Register file addressing}

Since most iterative HOF instructions typically operate directly on memory, the MPE register file is purposely made small to reduce hardware cost. The HOF concept tries to avoid unnecessary copying between mem- 
Listing 11.2: Basic implementation of the vector operation $d=a \cdot b-c$.

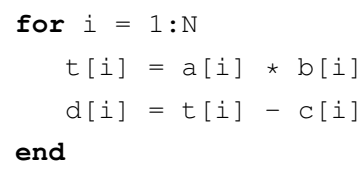

ory and register file to reduce energy consumption. However, the register file may sometimes be useful for temporary storage of data or memory addresses. Most architectures explicitly state which register to be used in the instruction word. This limits the number of registers because the instruction word should be kept short. Various ways of increasing the number of registers for such architectures have been proposed, such as using windowed register files or by addressing the register file in a similar way as memory $[116,117,118]$.

The MPE implements register file addressing, not to increase the size of the register file, but rather to be able to exploit multi-cycle HOF instructions when the register file is used as an instruction operand. One common use case is to be able to perform loop unrolling to hide datapath latency, in which case register file addressing can avoid increases in code size and instruction fetching.

\subsection{Element-based hardware repeat}

Element-based hardware repeat is a feature that allows the register file to be used for loop unrolling without associated drawbacks. It's characteristics will be demonstrated by the following example:

Consider the code in Listing 11.2. It represents the element-wise vector operation $d=a \cdot b-c$, which is frequently used in matrix operations [119]. Since the operation requires three input operand vectors $(a, b$ and $c$ ), the operation must be split into two instructions and a temporary (vector) variable $t$ is created. This code has a major performance issue: the data dependency between the two statements in the loop. 
Listing 11.3: Unrolled implementation, unroll factor $=2$

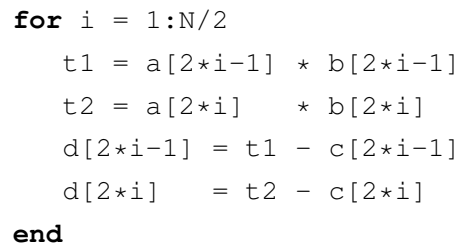

Listing 11.4: Pseudocode after loop distribution. By splitting the original loop in two, each new loop can be directly translated into a single HOF instruction, for a very simple HOF implementation.

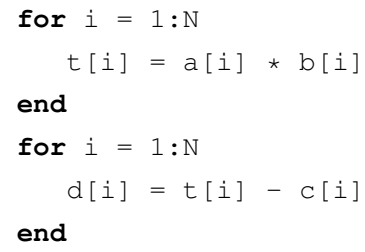

Performance can be improved in several possible ways. One typical approach is to use loop unrolling, as shown in Listing 11.3. The temporary variables $t 1$ and $t 2$ could be placed in registers to avoid memory operations and this approach is easily extended to SIMD instructions. Drawbacks include an increased code size and higher register utilization. An additional problem is that the code only operates correctly if the value $N$, the length of the vectors, is a multiple of the unrolling factor (and SIMD parallelism if the code is SIMDized). To handle cases when this is not true, additional code has to be inserted ${ }^{1}$.

A straight-forward solution that can better utilize HOF instructions is to use loop distribution (fission) [67], as shown in Listing 11.4. Each for-loop can now be implemented with a single HOF instruction. However, unless the vectors are short, the temporary vector $t$ has to be stored

\footnotetext{
${ }^{1}$ We assume that $N$ is not a compile-time-known constant. This is commonly occuring, since many kernels can be made more general if the number of elements to process can be passed at run-time.
} 
Listing 11.5: Pseudocode for the behavior of the loop implementation when using element-based hardware repeat.

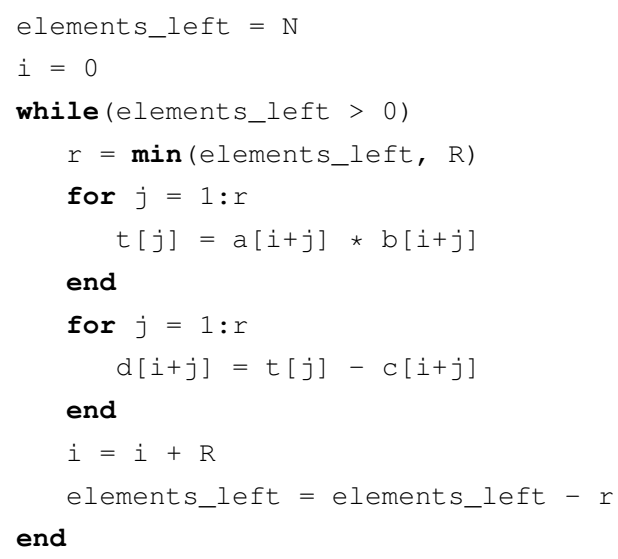

in memory, which increases the number of memory operations and thus data memory energy consumption. Furthermore, it assumes that the processor can perform three (vector) memory accesses every cycle to avoid a memory bottleneck.

To avoid the memory issue, but still allow the use of HOF instructions, a special hardware repeat mode can be implemented, that considers the number of elements to process, rather than the number of loop iterations to perform. Instructions within the loop get access to the number of elements to process via a special purpose register. Pseudocode for this operation is shown in Listing 11.5. The processor hardware would behave as follows: The element-based repeat instruction is passed $N$ (the number of elements to process) and $R$ (the block length). The processor exposes how many elements to process in the current iteration, $r$, through a special purpose register. The value of $r$ is equal to $R$, except in the final loop iteration, where $r$ may be different from $R$ if $R$ doesn't evenly divide $N$. Each of the two inner for-loops can be implemented with a single HOF instruction, which uses the value of $r$ to decide how many elements to process. Since the vectors now how been split into chunks of $R$ elements, the temporary vector $t$ may be stored in the reg- 
Listing 11.6: MPE assembly implementation of the example loop for 16-bit fixedpoint data and a 64 element block size. Special-purpose register el0 exposes the number of elements to process in each loop iteration. The syntax $v r++=0$ is used to set a vector register file pointer to 0 and then auto-increments the pointer each clock cycle for the duration of the instruction.

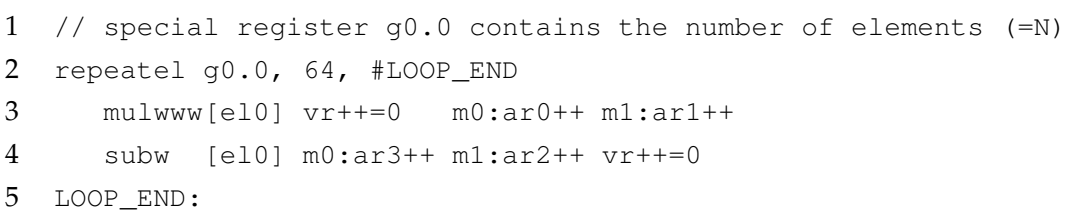

ister file, if $R$ is selected appropriately. Compared to memory-only HOF instructions, the vector length becomes quite limited due to the small size of the register file, which will increase instruction issuing. On the other hand this may be well compensated energy-wise by fewer data memory accesses. The register file of the basic MPE configuration with 8 vector registers is still enough to let a $\mathrm{HOF}$ instruction execute for at least 8 cycles, and the instructions of a small loop are easily cached by an instruction buffer/loop cache.

The assembly code corresponding to the pseudocode from Listing 11.5 is given in Listing 11.6. It demonstrates both the use of the elementbased repeat instruction, as well as register file addressing. In contrast to memory addressing, the register file address register can be set immediately using the $v r++=X X$ syntax, which sets the address to $X X$. Both memory and register file accesses use the ++-syntax, which indicates consecutive addressing of element over possibly many cycles.

It is of course of high interest to see the practical effect of elementbased hardware repeat and register file addressing. The example code has been implemented in several variants which have then been simulated in gate-level simulation, to gather performance and energy statistics. Relatively large vectors were used, with $N=4096$, to minimize the effect of the setup- and kernel termination code that was used in the implementations. Table 11.3 compares the different kernel implemen- 
Table 11.3: Multiply- and subtract kernel implementation comparison, where $N$ is the number of elements of a vector and $R$ is the element-wise repeat block factor. All versions perform 8-way SIMD/vector processing.

\begin{tabular}{l|cccc}
\hline \hline Implementation & LVM & $\begin{array}{c}\text { Insn. } \\
\text { fetches }\end{array}$ & $\begin{array}{c}\text { Code size } \\
\text { [bytes] }\end{array}$ & Constr. \\
\hline Basic & $6 N$ & $\sim N / 4$ & 96 & $\mathrm{~N} / 8=0$ \\
Unrolled, factor=1 & $4 N$ & $\sim N / 4$ & 64 & $\mathrm{~N} / 8=0$ \\
Unrolled, factor=2 & $4 N$ & $\sim N / 4$ & 80 & $\mathrm{~N} / 16=0$ \\
Unrolled, factor=4 & $4 N$ & $\sim N / 4$ & 112 & $\mathrm{~N} / 32=0$ \\
Unrolled, factor=8 & $4 N$ & $\sim N / 4$ & 176 & $\mathrm{~N} / 64=0$ \\
HOF-simple & $6 N$ & $\sim 2$ & 96 & None \\
HOF+HW.repeat & $4 N$ & $\sim N /(R / 2)$ & 80 & None \\
\hline \hline
\end{tabular}

tations, where Basic is the implementation from 11.2, Unrolled is based on Listing 11.3, HOF-simple is based on 11.4 and HOF + HW.repeat executes the code of 11.6. Note however that compared to the listings, the Basic and Unrolled implementations have been SIMDized, whereas the HOF-versions are naturally executed in SIMD fashion by the hardware. The SIMDization of Basic and Unrolled puts constraints on how many elements can be processed without additional code, where $N$ must be a multiple of both SIMD-width and the possible unroll factor.

Figure 11.3 compares the relative energy consumption, cycle time and code size of the different implementations. The kernel implementations Unrolled-8, HOF-simple and HOF-HW.repeat all reach approximately $98.3 \%$ of theoretical peak performance. The multiplications and subtractions should each consume 4096/8 = 512 clock cycles when using 8-way processing, for a total of 1024 cycles. The overhead is due to some setup code and pipeline latency at kernel termination. Data dependencies reduce the performance of the remaining implementations. Energy consumption is dominated by LVM memory consumption in all implementations, which is above $60 \%$ for all kernels. The two main competitors, Unrolled-8 and HOF $+H W$.repeat, represent the best implementations of 


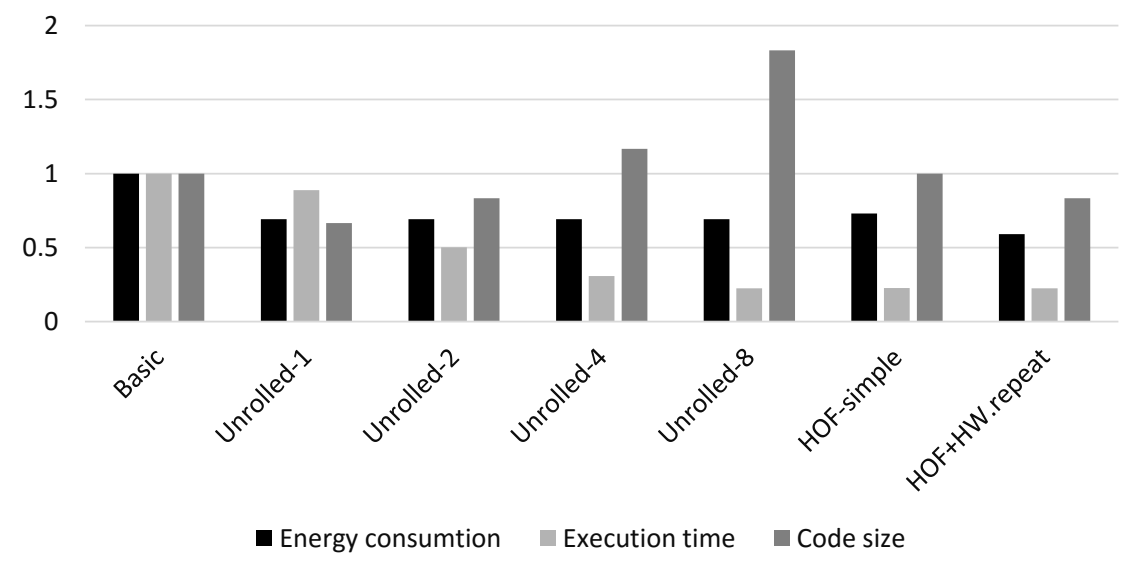

Figure 11.3: Comparison of relative energy consumption, cycle time and code size of the different kernel implementations.

SIMD execution and HOF execution respectively. Their performance is identical, however the HOF implementation reduces non-LVM energy consumption by $36 \%$ and total energy consumption by $15 \%$ compared to Unrolled-8. Additional, the code size is reduced by $55 \%$ and there are no restrictions on how many elements can be processed, whereas Unrolled-8 requires that $N$ can be evenly divided by 64 .

\subsection{MPE control}

The final topic for discussion is how to control the MPE. We typically assume that kernels can be executed independently on the MPE once they have been started. In this way, the MPE can be seen as a black-box, which simply returns a result when finished. This means that the performance of communication is of little importance for time-consuming kernels. However, for shorter kernel executions it is vital that the communication overhead between an MPE and the cluster main processor is as small as possible.

Figure 11.4 depicts the hardware that is shared between a CCC and 


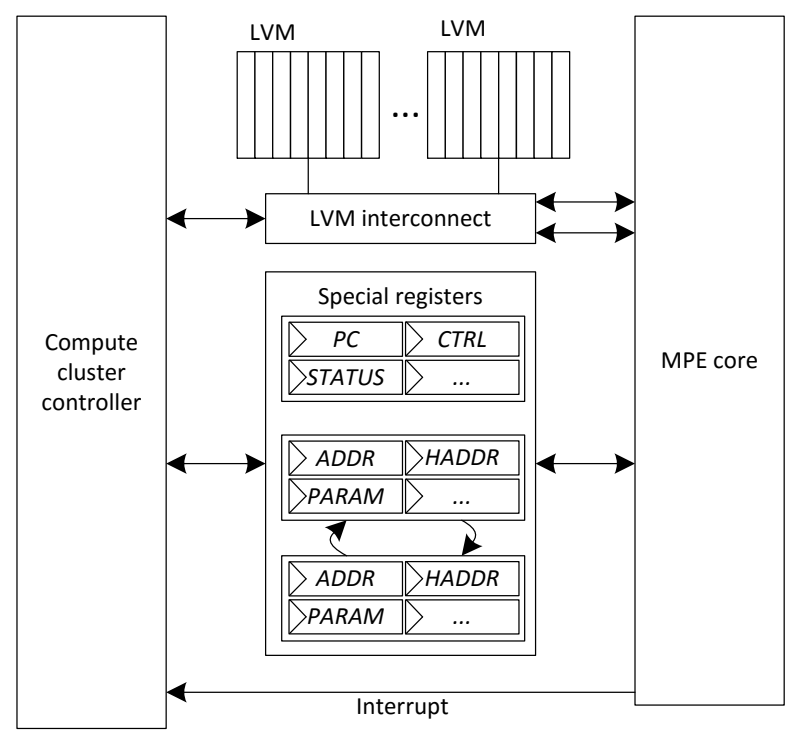

Figure 11.4: Cluster controller (CCC) and MPE communications hardware.

an MPE. Since the LVMs are shared resource, data exchange can be done through memory. However, an LVM is exclusive to whatever execution resource it has been assigned to and can only be reassigned by the CCC. Reassignment during MPE kernel execution may lead to race conditions and should not be used. The LVMs are therefore not suitable for direct communication. Instead, the main method of communication is to read/write the shared special purpose registers. Additionally, the MPE can generate interrupts to the CCC if configured appropriately.

Many of the internal registers in the MPE are mapped to the special register file that is visible to the CCC. It includes parameter registers, address registers, addressing help registers, status registers, configuration registers, the program counter and other registers. By sharing these registers, the CCC can pass parameters, setup pointers to LVM memory and deliver the address of the program code of the desired kernel. At the same time as the program code address is delivered, the CCC can set the kernel execution mode, select which memory consistency mode to use, 
enable or disable interrupt generation and decide if permutation- and constant data caches should be flushed.

A subset of the special registers, most notably the parameter registers and addressing registers, may be used in a rotating fashion. This means that while some registers are used by an active kernel, the CCC can simultaneously setup new address register for later kernel executions. This can be used to minimize the potential inactive time of an MPE.

During kernel execution, the CCC may poll arbitrary registers, but most importantly the status register, which indicates whether or not kernel execution has terminated. However, modifying a register which is actively used by an active kernel may have unexpected consequences and must be done with care.

The interrupt capability may be used if desired. It can be used to signal kernel termination or be triggered arbitrarily by the kernel software through interrupt instructions. Interrupts can also be used for error handling. The MPE tracks several sources of possible errors, such as illegal memory addresses, bank conflicts, floating point exceptions and more.

\subsection{Summary}

This chapter described the architecture of the ePUMA MPE, a practical HOF-based architecture with the proposed memory subsystem and datapath structure. Its specification was given and the hardware characteristics were presented. Many practical aspects of a HOF implementation was discussed, such as the non-atomicity of HOF instruction execution and the memory consistency model. To be able to use the register file for multi-cycle HOF execution, register file addressing and element-based hardware repeat was introduced, which may help save energy and reduce code size. The impact of all features in relation to the kernel library will be discussed in Chapter 15 . 


\section{Part IV}

\section{System configuration and programming}





\section{Chapter 12}

\section{Platform generation}

This chapter will cover the topic of how to generate a customized ePUMA platform. Many parts of the ePUMA platform creation process have been automated, which helps to reduce manual labor, but also simplifies design space exploration, since we can easily generate multiple platforms and compare them. However, before any platform can be generated, one should first decide what will be required by the final platform, such as an approximate compute capability and memory requirements. This is a non-trivial step, since the ePUMA platform has many configurable parameters and virtually any kind of custom accelerator can be added. We will start this chapter with some general discussions about different applications and what we may expect from a final platform.

\subsection{Usage scenarios}

A complete DSP platform may look quite different depending on the application requirements. This section will try to divide applications into a few different categories, based on their overall computing behavior. Based on these categories, we can make some interesting observations that can be guide the platform selection process. 


\subsubsection{Massively parallel processing}

The ePUMA platform has been designed to allow massively parallel processing. It can be configured with multiple compute clusters, each with multiple processors and such a platform can efficiently execute massively parallel computing tasks. The compute clusters may be homogeneously configured to allow the same kind of task to run on any core. Input and output data is probably stored in off-chip memory. Such a platform is well suited for applications such as image processing [40].

One of the main challenges to solve in massively parallel processing scenarios is how to manage off-chip main memory bandwidth. If the compute-to-bandwidth ratio is low, main memory bandwidth will be the limiting factor of the platform. One possibility is to carefully adapt the platform based on the compute-to-bandwidth ratio of the target application, which is to choose the number of cores based on the available bandwidth or scale the bandwidth. If the compute-to-bandwidth ratio varies significantly, one may use a larger number of cores when appropriate and power-gate cores when all cores cannot be used. Managing bandwidth will probably be one of the most challenging aspects for the software developer and one may greatly benefit from bandwidth-friendly algorithms.

\subsubsection{Dataflow DSP}

Many DSP applications can be described with the dataflow programming paradigm, where the application is described as a directed graph of processing steps [120,121]. For our purposes we can consider each processing step or node in such a graph to be a compute kernel. This kind of processing can be implemented in several ways. If the kernels are diverse and/or some computing steps are best implemented with accelerators, one can heterogeneously configure compute clusters for their specific task(s) in the processing chain. The ePUMA ring network, described in Section 5.2.3, has been designed with this scenario in mind and allows high throughput data transfers between compute clusters. Inside 
the clusters, data can be exchanged between processors and accelerators with a simple memory reassignment. By doing so, one can avoid intermediate data storage in main memory, which reduces the main memory load and reduces energy consumption. If the tasks instead are more homogeneous, smaller chunks of input data could possibly be assigned to different clusters in a round-robin fashion, to avoid inter-cluster data transfers.

\subsubsection{Application-specific DSP}

ePUMA can be used as a main compute resource for applications with tasks of very different needs. A compute cluster can be dedicated to a particular task and task-specific accelerators can be attached to the cluster. The instruction set for MPEs on different clusters could differ. The programmers would still benefit from a streamlined programming model common to all clusters. All clusters and applications would be programmed in the same way, while still having application-specific hardware. Only a single tool-chain for application development has to be learned. Also, common kernels only have to be developed once. This allows for good code reuse, simplified development and less overall kernel development and verification time.

\subsubsection{General DSP}

Although ePUMA is primarily designed as an application-specific DSP, there is nothing preventing ePUMA from being used in a more general setting. A general platform in this sense is a platform that is not designtime optimized for any given application. Such platforms are quite common and general DSP platforms are still much more energy-efficient for DSP computing than e.g. general-purpose CPUs or GPUs.

A general DSP platform is likely to be homogeneously configured. It may lack application-specific accelerators, but on the other hand it could possibly include more memory, a broader instruction set for many data types, homogeneous compute clusters and MPEs and more general in- 
terconnection networks. It may use more chip area and consume more power, but should be able to target a wide range of applications with decent performance and energy efficiency. This configuration is probably desired for applications which have a wider range of workloads, or when the desired hardware capabilities are not known at design time.

\subsection{Architecture selection}

With a large set of tunable parameters, one must ask the question: How should the parameters be selected to achieve the most suitable architecture for a given application? The following process sub-divides the task into smaller and more manageable steps:

1. High-level application modelling: The application is modelled at a high-level to identify critical subtasks and give an initial estimate on an appropriate task allocation.

2. Algorithm selection/adaptation: Select the appropriate algorithms to implement the application and kernels, by taking the capabilities of the target platform into account.

3. Task allocation: Decide how and where to run each algorithm, that is if it should be performed by an accelerator, a single core or multiple cores. Existing kernels in a kernel library may be greatly aid this process.

4. Software/accelerator implementation: Implement the system-level software, compute kernels and possible accelerators.

5. Benchmarking: Benchmark the application! This may be done on a simulated platform with very generous system parameters. Determine the actual instruction usage, memory usage, core usage, cluster internal communication patterns and NoC-level communication patterns. Estimate requirements for possible future expansion. Based on this, the hardware requirements for the final platform are derived. If the performance is not satisfactory at this stage, 
one may have to move back to Algorithm selection, to tune the implementation.

6. Generate final platform: When the benchmarking phase is completed, we have derived our platform specification. The final platform can then generated.

\subsection{Platform creation process}

The ePUMA platform creation process is summarized by Figure 12.1. The dotted nodes represent automated steps, which are performed the ePUMA platform builder tools. These tools take a large set of platform parameters and simply generates the hardware platform and programming tools, including simulators, RTL code, assemblers and more. The process starts from a platform specification, which can be derived as described in Section 12.2. The platform specification can be broken down into a system specification, one or multiple MPE specifications and accelerator specifications. This allows parallel component creation, kernel/accelerator development and evaluation. The components can then be integrated into the full system, as soon as they become available.

An MPE is created by choosing an appropriate hardware setup, including instruction set selection and possible creation of custom instructions. The MPE datapath is optimized as described in Section 10.4. Once the datapath is finalized, the MPE builder tool generates tools such as an MPE simulator, an assembler, a code optimizer and C-files for system/kernel-interaction. Finally, the MPE RTL code is generated.

Accelerator implementation primarily involves the creation of an accelerator simulator and RTL code. Since this is fully custom hardware, there are no strict rules about how this should be done. However, to integrate the accelerator with the ePUMA system simulator and RTL code, the accelerator should be compatible with the ePUMA accelerator interface.

Typical ePUMA system configuration parameters were already introduced in Tables 5.1 and 5.2. It includes parameters such as the number of 


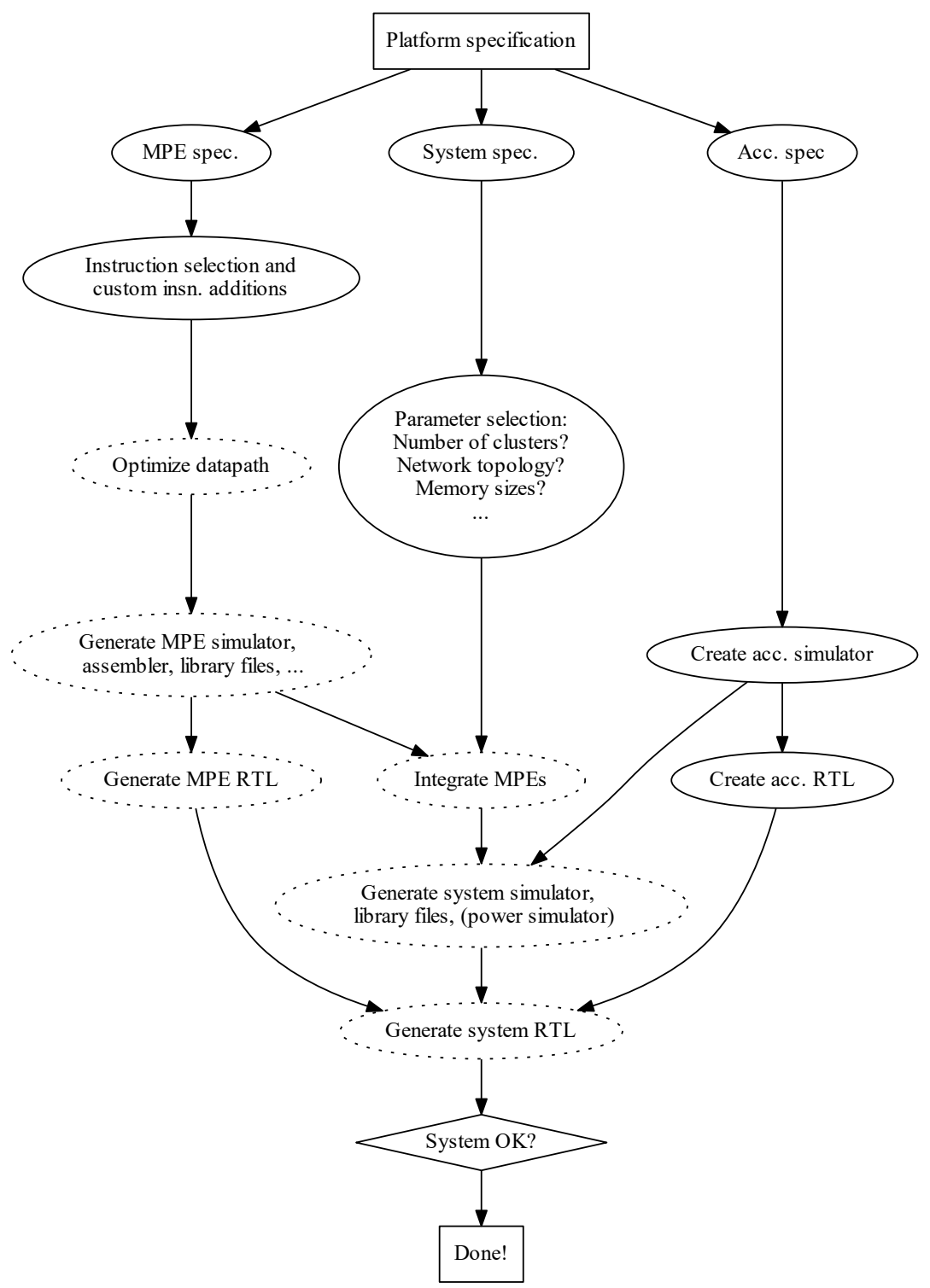

Figure 12.1: ePUMA system creation flow. Dotted nodes represent automated steps in the creation process. 
clusters, where each MPE configuration should be used, network topologies and more. The ePUMA platform builder uses code generated by the MPE builder to integrate MPEs into the ePUMA design and then finally generates the system tools and final RTL code.

Once all these steps are complete, a platform is fully generated. One must then decide if the generated platform is satisfactory or not, and possibly make modifications. At this stage one will also want to perform hardware synthesis, to make sure the platform has a satisfactory hardware cost and can reach the desired clock frequency.

\subsection{Summary}

This chapter briefly described some different system configuration scenarios and how to select and generate an ePUMA platform. The critical part is platform selection, which may have to be iterated multiple times during design space exploration. Once a suitable platform specification has been decided, the ePUMA platform builder tools automate the generation of simulators, programming tools and RTL code. The platform is made application-specific through its configuration and instruction set selection, while the rest of the platform generation is conveniently automated. 


\section{Chapter 13}

\section{Kernel programming}

This chapter will discuss kernel programming in general and specifically the implementation of compute kernels for the ePUMA MPE. Kernel programming is not only the process of creating the actual code, but also about adapting a high-level behavioral description of some algorithm to execution under limited data word length and limited memory space. Finally, the eOctave language will be introduced, a kernel programming language that has been co-designed with the architecture of the ePUMA MPE.

\subsection{Goals}

The target applications of ePUMA are embedded applications with extreme efficiency requirements. This is not specific to ePUMA, but to most DSP processors. In this domain, an increased programming effort may be acceptable to meet application requirements. Assembly programming is frequently used to meet such goals, at least for the most critical compute kernels. Architectures that claim to be $\mathrm{C}$ programmable often do not produce high quality code without the use of a significant amount of compiler directives, intrinsics or other architecture-specific functions, which brings the level of programmer-provided detail close to assembly programming anyway. We would like to explore the possibilities of eliminating assembly programming by other means. With high-level machine 


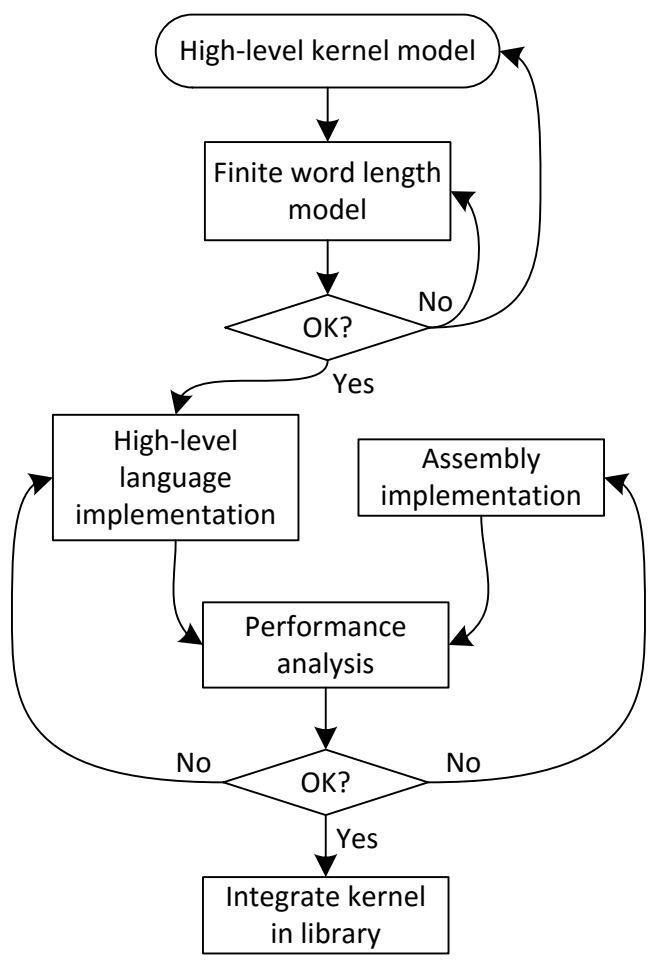

Figure 13.1: A simple DSP kernel implementation flow.

instructions such as HOF instructions, one can ask oneself: Is it possible to generate high quality machine code through the use of high-level code constructs and simple programming guidelines?

\subsection{Kernel programming flow}

In the best case, a DSP platform is shipped with all required kernels of any application, kernels which have been optimized by a platform expert. Since this often may not be the case, additional kernels have to be implemented. A simple kernel programming flow that can be applied to any DSP processor is shown in Figure 13.1. One of the most important steps is to transfer a high-level description of an algorithm into a finite word length model. Although many DSP processors support long word 
length computing, the extra time spent to adapt an algorithm to a limited word length will improve energy efficiency, processing speed and storage density. Some algorithms are easily converted, while others require significantly more effort. Once a suitable finite word length model has been derived, one may use a high-level language to implement the kernel. If performance is good enough, one may keep this kernel implementation, but in practice one may want to optimize the kernel in assembly for even better results. The main drawbacks of assembly are the increased programming effort and the non-portable code. Even if two processors share the same instruction set, an optimal assembly implementation for one of them may not be the best implementation for the other.

\subsection{Related work}

To avoid assembly programming, the best approach is possibly to codesign a compiler with the target architecture or target application domain. This has been attempted by many before and may generally produce better results than other approaches [70, 122, 123].

Previous work regarding kernel programming of the ePUMA MPE include the FT language and its compiler [8]. The main purpose was to demonstrate how the 2D addressing features of the MPE could be applied automatically, through the use of dataflow analysis [124]. There are also several publications which deal with the issue of finding conflict-free parallel memory access patterns for the multi-banked architecture of the LVM memories [46, 47].

Other previous work related to the ePUMA MPE relates to the problem of instruction selection, specifically to see if it is feasible to automatically apply complex MPE instructions [125]. Although possible, in general this may be complicated and time consuming. There is also ongoing research which deals with code generation for the multi-bank memory architecture of the MPE, using constraint programming [126]. 


\subsection{The eOctave kernel programming language}

This research introduces the eOctave programming language, a simple demonstration of how a high-level language may be co-designed with the concepts of HOF instructions and conflict-free parallel memory access of multi-bank memory.

\subsubsection{Motivation}

The $C$ programming language is popular in many areas computing, including DSP computing. However, the level of abstraction is very low. Furthermore, the language leaves the behavior of much potential code undefined, and other potential problems such as aliased pointers may prevent the use of static analysis or automatic vectorization/parallelization. In order to provide stronger guarantees, some other approach is desirable.

An additional problem with the low-level programming of $C$ is that it may hide the original intent of the programmer. It makes little sense to let the programmer express common operations such as dot products, convolutions and even FFTs using only basic operations such as addition and multiplication, only to let the compiler try to find the programmer's original intent through complex compiler analysis. This process may not even succeed [24] and leads to an unnecessarily complex compiler implementation. Instead we would like to assume that the programmer is fully aware of what operations he/she intends to implement, but is less eager to learn too much about architectural details. The hope is that only a few simple programming rules should be required in order to produce high-quality machine code.

DSP programmers are typically well-familiar with algorithm prototyping in languages such as Matlab, Octave or Scilab. A programming rule that has well served programmers of these languages over the years, is to express code as much as possible using vector/matrix operations, principally the larger the better, and to use built-in functions instead of manual implementation for common operations. By doing so, the per- 
formance is likely to be good. In contrast, loops and scalar operations may perform very poorly. Performance may not always be significant for programs in these languages, since the main purpose of such program is typically prototyping. However, the same programming guideline makes very much sense when compiling for a vector architecture, because it may simplify the translation into vector instructions. The main idea behind eOctave is then that an algorithm prototype written in Matlab/Octave should be easily translated into eOctave, using very much of the same syntax, and if the guideline of using vector/matrix operations and built-in functions is followed, performance should be good.

\subsubsection{Design goals}

The main design principles that directed the implementation of eOctave are the following:

- Syntax: Use a syntax similar to Matlab/Octave, to create a sense of familiarity and to make manual code translation from Matlab/Octave to eOctave relatively straight-forward.

- Explicit typing: Since the use of appropriate data types is important to DSP computing, data types should be declared explicitly.

- Data placement: The possibility to specify the data distribution over multiple memories is required. An additional required feature is the possibility to describe patterns of conflict-free parallel memory access, such as the use of padding.

- Built-in high-performance functions: Provide built-in functions that translate into high performance code. Built-in functions such as FIR-filtering or FFT may be implemented in the language itself or assembly programming as desired.

- Vector/matrix operations: To produce high performance code the use of vector/matrix operations and built-in functions is required, in much the same way as Matlab/Octave programmers may expect. 
- C->eOctave interface: Make it possible to specify the required information so that a C-program can call the kernel appropriately, by generating a $\mathrm{C}$ kernel wrapper. In case of ePUMA, this wrapper will execute on the cluster controller, which will then initiate execution on an MPE.

These principles by no means guarantee that assembly programming is eliminated. In fact, the implementation of built-in functions may still be performed using assembly, although the functions are provided by a platform expert. Such functions may hide multiple assembly-level implementations for different target architectures. By gradually expanding the language with more built-in functions and other functionality, the goal is instead to gradually hide assembly programming from the system designer.

\subsubsection{Design methdology}

The design of eOctave has followed a procedure which is very much based on the kernels that we wish to implement. Many MPE kernels were originally implemented in assembly. The translation into high-level kernel implementations followed the following procedure:

1. Choose an already existing assembly kernel for high-level implementation.

2. Add the necessary features to eOctave to allow a high-level implementation.

3. Optimize the compiler code generation process until the same performance is achieved by both the assembly and the high-level implementation.

The last step is the most important one. It is considered of extreme importance that the compiler principally does not add any overhead compared to a hand-tuned assembly implementation. If this is not the case, the original assembly implementation may simply be wrapped in a built-in function for better results. The development methodology above 
Listing 13.1: A simple eOctave kernel.

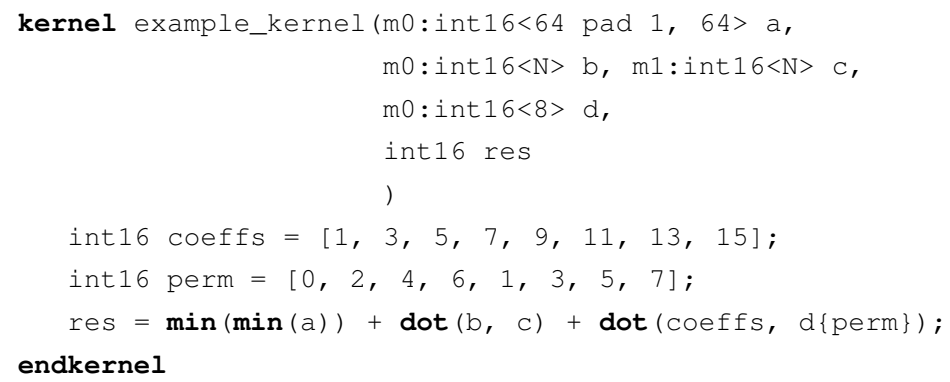

clearly means that the language and the compiler are implemented iteratively. This has allowed us to concentrate on solving the code generation issues of the real-world kernels of most importance.

\subsection{4 eOctave kernel example}

Since a full language specification and all details of the code generation process is outside the scope of this thesis, the most important aspects will instead be demonstrated through a kernel example. The example is shown in 13.1. The kernel starts with the kernel keyword, followed by the kernel name and kernel arguments. Multiple input data vectors are specified, where $m 0 / m 1$ specifies which input memory the data is located in, int16 represents the datatype (16 bit integers), after which the number of elements is given. As shown in the example, the number of elements must not be fixed and can instead be given as a parameter. However, the compiler performs static analysis to check that operations are legal. Since input vectors $b$ and $c$ are combined in a dot product, they must be of equal length, and consequently both must be specified with the same input parameter $N$, representing the number of input elements. Input data structure $a$ is a $64 \times 64$ matrix. The use of pad 1 indicates that each row has been padded with a single dummy element.

The kernel example defines two constant vectors, coeffs and perm, where the first is used as constant data and the other as a permutation 


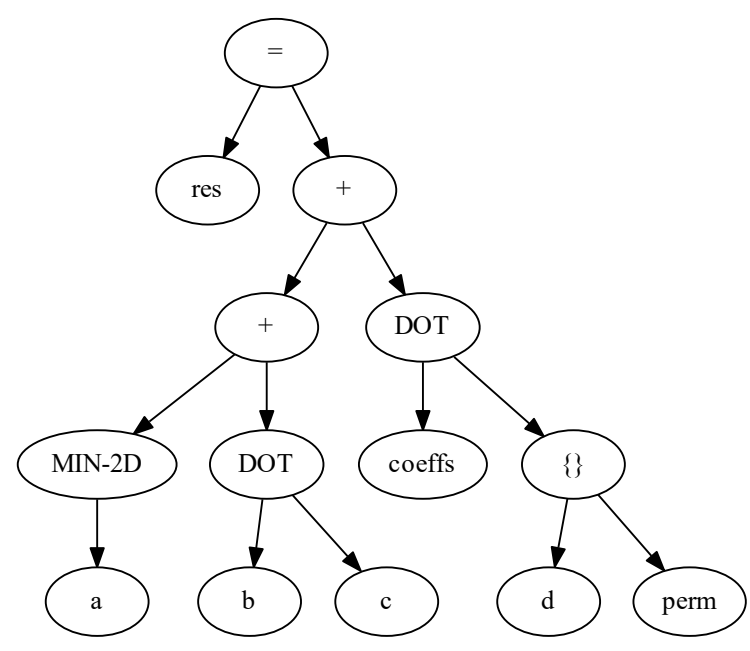

Figure 13.2: Intermediate representation of the main computation in the example kernel.

vector, both of which will be allocated to the MPE local store. The kernel then continues by specifying a very simple computation, that is to compute the minimum value of matrix $a$, the dot product of the two vectors $b$ and $c$, followed by the dot product of the constant coefficient vector coeffs and input vector $d$, to which a permutation pattern is applied. The use of curly brackets instead of normal parenthesis for the indexing operation $d\{$ perm $\}$ is used to separate indexing operations from function calls.

The intermediate representation (IR) used in the compiler is shown in Figure 13.2. Due to space limitations, only the main computation is shown. The IR is not lowered beyond this point, because the same operations are available in the MPE instruction set. The use of $\min (\min ())$ is identified as a two-dimensional minimum operation, since this can be described by a single HOF instruction. Other similar code such as $\max (\max (a)), \operatorname{dot}(a, \operatorname{conj}(b))$ and $a b s(a) . * b$ may also be identified and translated into single HOF instructions.

The MPE assembly code generated by compiling the kernel exam- 
ple is given in Listing 13.2. The code starts with a section between labels KERNEL_TEST and KERNEL_TEST_END. This code is only used to setup kernel input parameters, so that the MPE can be simulated in a standalone environment. In a full system however, this is instead performed by a compute cluster controller and this code can then be discarded. Likewise, the labels which include KERNEL_DATA are used by the test code to define where the input data is located in LVM memories for standalone simulation.

The main kernel code is located between the labels KERNEL_MAIN and KERNEL_MAIN_END (lines 11 to 20). Line 12 performs the operation $\min (\min (a))$. The syntax $(+=1)$ indicates that one element is skipped after each block has been processed, to account for the padding of matrix $a$. Line 13 performs the dot product of vectors $b$ and $c$, using the instruction value register ivr 0 to supply the number of elements to process. Line 17 performs the last dot product using constant data and a permutation vector from the local store. Just before the final dot product is performed, address pointers to the local store are setup. Lines 14 and 18 perform the summation of all three sub-results and the output value res is placed in a general-purpose register $g 0.0$, which is shared with the CCC.

The code generation process is currently rather simple, as it simply expands the intermediate representation into the final assembly code with few optimizations. This results in code which is poorly scheduled. For example, the local store address pointers can be set earlier to potentially avoid an address register data dependency. A separate tool, an assembly code optimizer, is used to reschedule the code in a postprocessing step ${ }^{1}$. Proper scheduling of instructions is naturally important, but its effect on performance is rather small as long as long vector instructions are generated.

In addition to the MPE assembly code, a C kernel wrapper is generated, which is shown in Listing 13.3. The kernel wrapper, executed by a CCC, sets up the necessary memory address pointers and starts execu-

\footnotetext{
${ }^{1}$ The assembly code optimizer tool was implemented earlier than the compiler and has been reused simply to reduce work effort. This processing step should eventually be integrated into the compiler itself.
} 
Listing 13.2: Generated MPE kernel assembly.

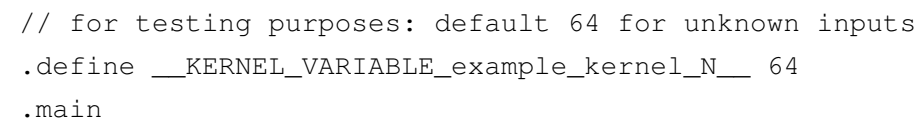


Listing 13.3: Kernel wrapper for the example kernel.

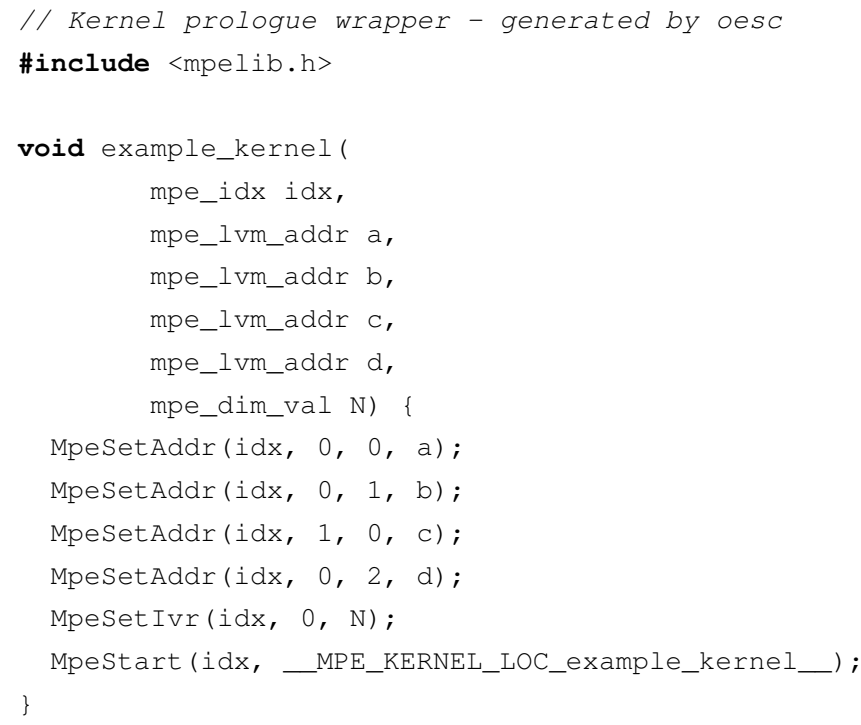

tion on an MPE. After starting the MPE, the function returns and MPE execution will continue in the background. To use the kernel, an application program simply calls the kernel wrapper, but some conditions must be fulfilled to ensure correct operation, such as

- The chosen MPE must be configured with the appropriate instruction set and memory configuration to be functional.

- The kernel code must have been loaded to the MPE local store.

- The MPE must have been assigned an appropriate number of LVM memories.

- All input data structures must be non-overlapping. The compiler assumes this is the case during code generation. This must be guaranteed by the system programmer. 


\subsubsection{Compiler implementation}

The compiler translates kernel code into a high-level intermediate representation (IR) which allows simple translation into corresponding machine instructions. Since most kernels operate directly on memory, register allocation is much less critical and instruction schedule optimization is performed in a post-processing step, currently by a separate tool.

The current implementation assumes that unused LVM space may be used arbitrarily, to e.g. allocate temporary storage buffers. Alternatively the kernel must be provided with pointers to memory which can be used for intermediate storage. This approach is common in shared memory DSP kernel libraries. Special attention must be given to input data structures of statically unknown size. Another important aspect which affects performance, is to determine which LVM memory a temporary data structure should be placed in for best performance. This can be determined with a simple heuristic or through optimization [8]. The current implementation simplistically assumes that best performance is achieved if the two input vectors to a binary operation are placed in different memories. For unary operations, known placement of either the input or the output data will decide the placement of the other potentially unknown operand. The placement of the input data structures are given by the kernel parameter list, which may resolve many placement decisions. If placement cannot be determined by these rules, a random choice is made. The programmer may explicitly decide the placement as well, if desired.

\subsubsection{MPE kernel library implementation}

Although the MPE kernel library originally has been implemented using assembly, a majority of it has now been transferred to eOctave. Because of the complex instruction set operations of the MPE, many common DSP kernels become single-instruction kernels and others are implemented by a smaller set of long vector operations. Some more complicated kernels are kept in assembly and may be used through function calls. Ma- 
trix decompositions, such as QRD, LUD or SVD, are a few such examples. These require significant optimizations to perform well and may even have to be optimized differently depending on matrix size. Likewise, kernels which use specific addressing modes, such as histogram generation, are still programmed in assembly. Implementation of more optimizations and more advanced addressing mode selection are topics for future research.

\subsection{Summary}

This chapter described the kernel programming process for DSP kernels, and specifically the creation of kernels for the ePUMA MPE. The eOctave kernel programming language and the eOctave compiler for the MPE were introduced through a simple kernel example. While still far from complete, the reasoning behind the language design was given. By keeping the level of abstraction of the intermediate representation at the level of HOF instructions, simple translation into machine instruction is possible without complicated analysis. If the kernel programmer implements kernels using large vector/matrix operations and built-in functions, kernel performance may approach that of kernels implemented in assembly language. 


\section{Chapter 14 System programming}

This chapter aims to briefly review system programming models for parallel processor architectures. Specific focus will be devoted to architectures with distributed memory such as ePUMA. The work with the system programming model for ePUMA touches almost every aspect of the ePUMA system, both core-level hardware, system-level hardware as well as the corresponding software tools. The topic has been given attention in previous work and the efforts have been collective $[7,8]$. This chapter thus summarizes all work so far and presents a simple ePUMA system application example.

\subsection{Parallel programming frameworks}

Since the introduction of multi-core processors, many different frameworks and APIs have been introduced to allow easier exploitation of multi-core and multi-processor systems. Although a lot of progress has been made, there is still much left to do. A parallel programmer faces many challenges, such as multi-core synchronization, estimating communication overhead and optimizing the use of the limited shared main memory bandwidth [127].

OpenMP is a parallel programming framework for shared memory multiprocessor systems, primarily for $\mathrm{C}, \mathrm{C}++$ and Fortran programming [128]. It allows parallel processing through pre-processor directives, by 
allowing the programmer to control which parts of the code should be executed in parallel or not. It is the programmer's responsibility to guarantee what code can be executed in parallel safely, which introduces a potential error source. It can still be considered as one of the simpler methods of parallelizing a program, but since it is primarily designed for homogeneous shared memory systems, it is not useful for distributed memory architectures such as ePUMA.

For GPU programming, CUDA [73] and OpenCL [129] are popular approaches. CUDA is exclusive to NVIDIA devices, whereas OpenCL can be used for multiple platforms [130]. Parallel processing is heavily influenced by the GPU architectures, which divides the workload between threads and thread blocks. Explicit synchronization or atomic operations have to be used by the programmer to ensure correct operation. Although these frameworks are interesting, and early investigations have been to use OpenCL on ePUMA, this approach has discontinued.

The bulk-synchronous programming (BSP) model could be useful for many parallel platforms [131]. The Cell Broadband Engine from STI is similar to ePUMA from several perspectives and the BSP model has successfully been used for this platform [132]. The main problem is that the BSP model may not work well for any application. Still, this model requires further investigation.

Since heterogeneous platforms are becoming increasingly popular, several frameworks address these architectures specifically. OpenACC (Open Accelerators) is one such initiative [133]. It has some similarity to OpenMP, including the use of compiler directives to guide code generation.

One of the most popular parallel programming systems which is similar to the current ePUMA programming methodology is MPI (MessagePassing Interface) [134]. It uses explicit send/receive-operations, which gives the programmer high control over data movement. It is suitable for both multi-processor systems and distributed systems. However, MPI uses a single program - multiple data (SPMD) programming style, which 
is not directly applicable to ePUMA. The SPMD style implies that the complete program code is available everywhere in the system, although only a smaller fraction of the total program may be executed on every node in the system. The current ePUMA platform requires that programs are moved explicitly, which does not translate directly to MPI. However, by doing some modifications to MPI, such as dividing the program into smaller tasks, MPI could still be applicable [135].

There are many software libraries available that can aid parallel programming in specific application domains, such as OpenCV [41] and TensorFlow [136]. The mapping of TensorFlow to ePUMA is currently under investigation. A TensorFlow application expresses the computations as a dataflow graph of kernels, which should excellently fit ePUMA. The kernel set is also similar to the ePUMA MPE kernel set. One possible use of this approach is to use ePUMA for machine intelligence in batterypowered vehicles, such as UAVs.

CAL is an interesting concept that has been investigated in collaboration with external parties within this research project $[137,138]$. This is still ongoing research. In CAL, actors are used to transform input data streams to output data streams for dataflow applications. The language does not directly specify whether it should be used for system or kernel programming, but the most promising approach is to let actors be MPE kernels and use CAL to schedule kernels over the ePUMA system.

Programming using algorithmic skeletons is a useful technique for distributed memory architectures [139, 140]. The idea is to implement general data-level parallel and task-level parallel computational patterns that can be used over many applications. An application programmer then simply uses the pre-defined patterns to create applications, while the architectural details of communication, synchronization and loadbalancing are hidden by the skeleton implementation. Algorithmic skeletons may also be used together with auto-tuning to optimize for execution time [141] or even energy consumption [142]. A common skeleton is the map skeleton, which is similar to the map pattern of HOF instructions. It allows data parallel execution over a complete system. The best use of 
such a pattern is not to apply a function to single elements, but rather to larger blocks of data, similar to the HOF block-map pattern. In this way one may find more work to do per loaded main memory data element and significant amounts of main memory traffic can be avoided. A library of ePUMA skeletons have been implemented for this purpose. The total utilization of all MPEs in an ePUMA system can approach $100 \%$ if the compute-to-bandwidth ratio is high enough and main memory doesn't limit processing speed. This is mainly because memory transfers can be performed in parallel with computing, and the master processor and cluster controllers can perform most of the control operations. One such successful experiment is the implementation of an MJPEG-based web camera [143].

\subsection{Kernel-based programming}

Many parallel programming frameworks claim that the main focus is kernel-based programming. This typically means that the most frequently executed code or innermost loops of an application are given the most attention. It makes sense to specifically target such code, since this is where most of the execution time is typically spent. In some contexts, kernelbased programming implies that compute kernels are offloaded to some specific hardware accelerator for faster execution, e.g. a GPU. This is also the case for ePUMA.

Kernel-based programming in the ePUMA context specifically means that application programming is based on the use of compute kernels, specifically MPE kernels. They are not necessarily the most frequently executed code or the innermost loop of some algorithm, although this is typically the case. Whatever code has been mapped to an MPE is called a compute kernel and the purpose of application programming is to exploit such code maximally, since execution should be faster and more energy efficient. Regardless of whether appropriate kernels are already available in a kernel library or if they have been implemented specifically for the application, a system programmer may regard a compute 
kernel as a black box, and principally only has to care about the kernel's applicability and execution time.

\section{3 ePUMA programming flow}

A simplistic top-down ePUMA programming flow is shown in Figure 14.1. This is the approach that has been used most often within the ePUMA project. It starts from golden application model, which is either already existing reference code or an application model written specifically for the ePUMA project. The application is then analyzed manually, in order to identify possible compute kernels. The complexity of this step varies significantly with the application and may have to be iterated several times before a satisfactory kernel set has been identified.

When appropriate kernels have been identified, kernels must be mapped over MPE cores and compute clusters. An automatic scheduler may aid this process, especially if the kernels have fixed execution times and communication overhead can be modeled appropriately [8]. Systemlevel code may then be written or generated, and kernels are introduced by inserting appropriate kernel calls.

It is often possible to reverse the programming flow, especially if no golden model exists. In this case, one builds an application bottom-up from already existing kernels by simply calling kernels from CCC code and finally setup the master code to schedule appropriate data transfers and overall control. In practice, the top-down and bottom-up approaches may be combined, which allows overlapped kernel-level and systemlevel software development.

\subsection{Programming tools}

For many DSP platforms, access to physical hardware is not required for application development. A system simulator can often simulate a platform fully or approximately. Depending on the level of detail, the simulator may give even more detailed statistics about performance be- 


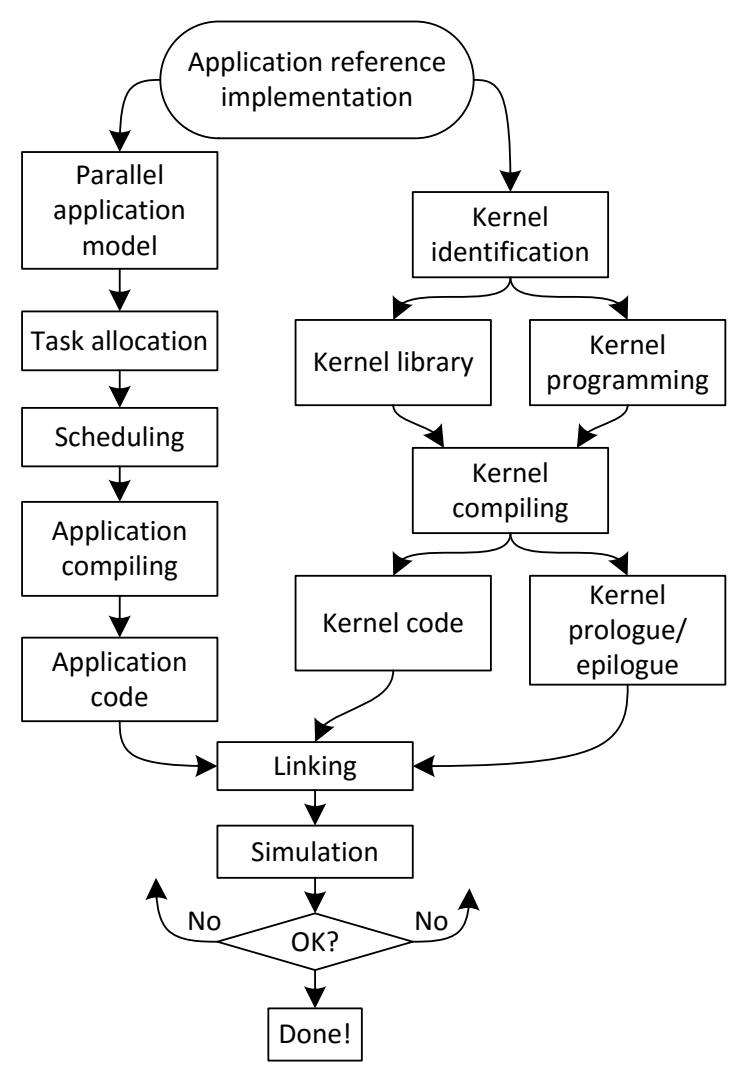

Figure 14.1: Top-down system programming flow.

havior than the hardware platform. The full ePUMA toolset includes a MPE kernel simulator, a system simulator, assemblers for all processors, $\mathrm{C}$ compilers for the master and compute cluster cores, an eOctave compiler for the MPE, power simulators for the MPE core and the complete system, a multi-core task scheduler and some other minor tools. The complete tool-chain set is given in Appendix A.

\subsection{Operating system support}

In this particular work, the ePUMA system is used baremetal. However, a simple light-weight operating system has also been implemented and 
may be used if desired [7]. The current master processor is also able to boot a Linux operating system without memory management. This creates many interesting opportunities, but it will not be further investigated in this work.

\section{6 ePUMA application example}

This section demonstrates how to use the current ePUMA system using the low-level ePUMA API. It is exemplified by running one FIR-filtering task on each available MPE in the system. This example system uses 8 compute cluster, each configured with 8 MPEs for a total of 64 MPEs. Listing 14.1 shows the code running on the master processor. It broadcasts the same compute cluster controller (CCC) program and MPE kernels to all CCCs and MPEs respectively, after which CCC execution commences. Input data is then loaded to all MPEs. After loading 64 input data sets, the master simply issues 64 store operations. In this case, each call to ClusterToMM will automatically synchronize with the corresponding compute cluster by halting until the compute cluster is ready to store results. This functionality is provided by the on-chip network.

The CCC code in Listing 14.2 is responsible for storing incoming data to LVMs, invoking kernels, waiting for kernel completion and then initiating writeback of filtering results to main memory. It controls data allocation over LVM memories and the data's location within each memory. In this particular example, it assigns two LVM memories to each MPE, one of which should contain newly arrived input data and the other which can be used to store output data.

\subsection{Summary}

This chapter discussed parallel programming frameworks and the current parallel programming methodology used for ePUMA. Although the main method for application development so far has been to use a lowlevel API, there are plenty of opportunities for further improvement. 
Listing 14.1: Master processor program.

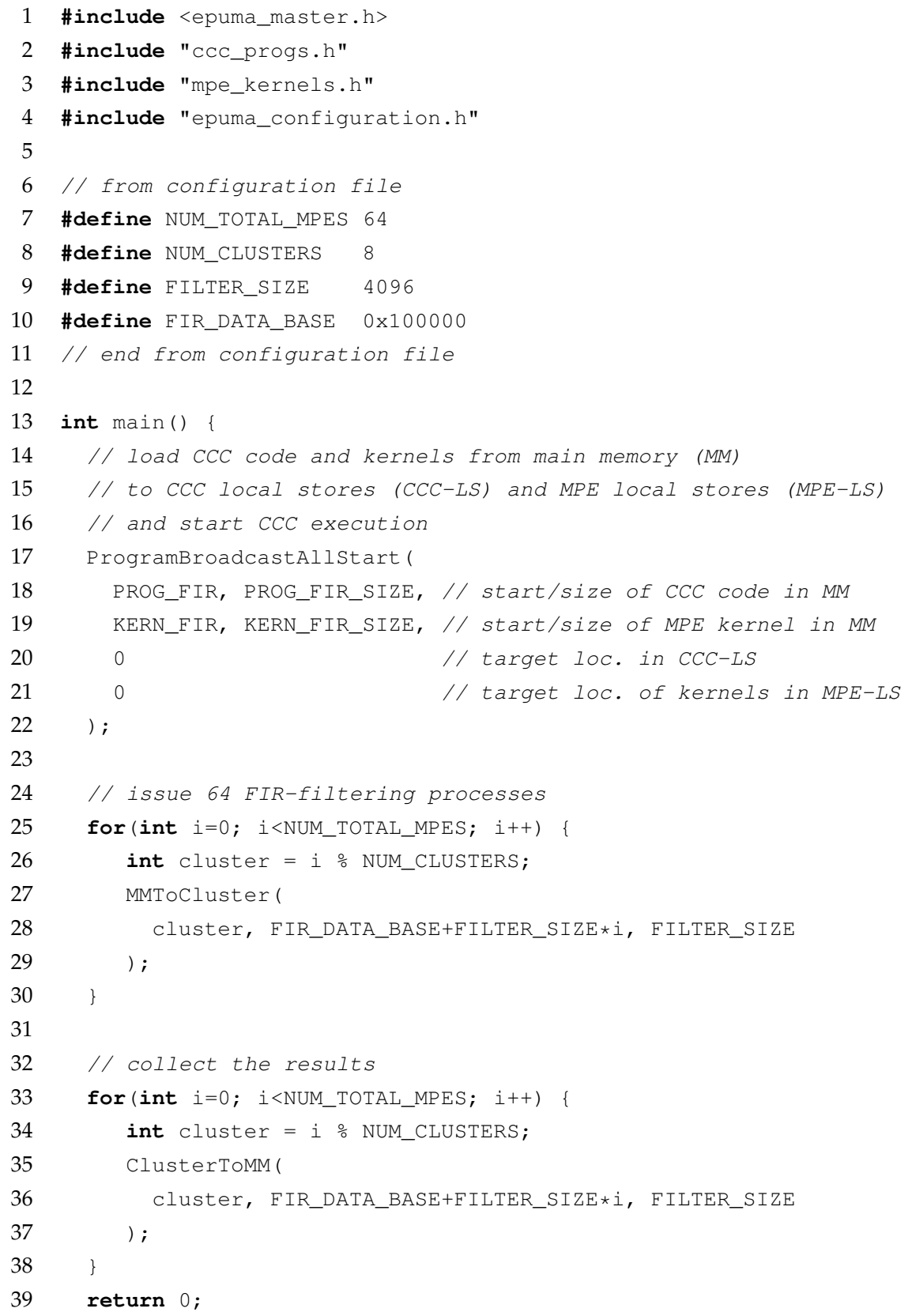


Listing 14.2: Compute cluster controller program.

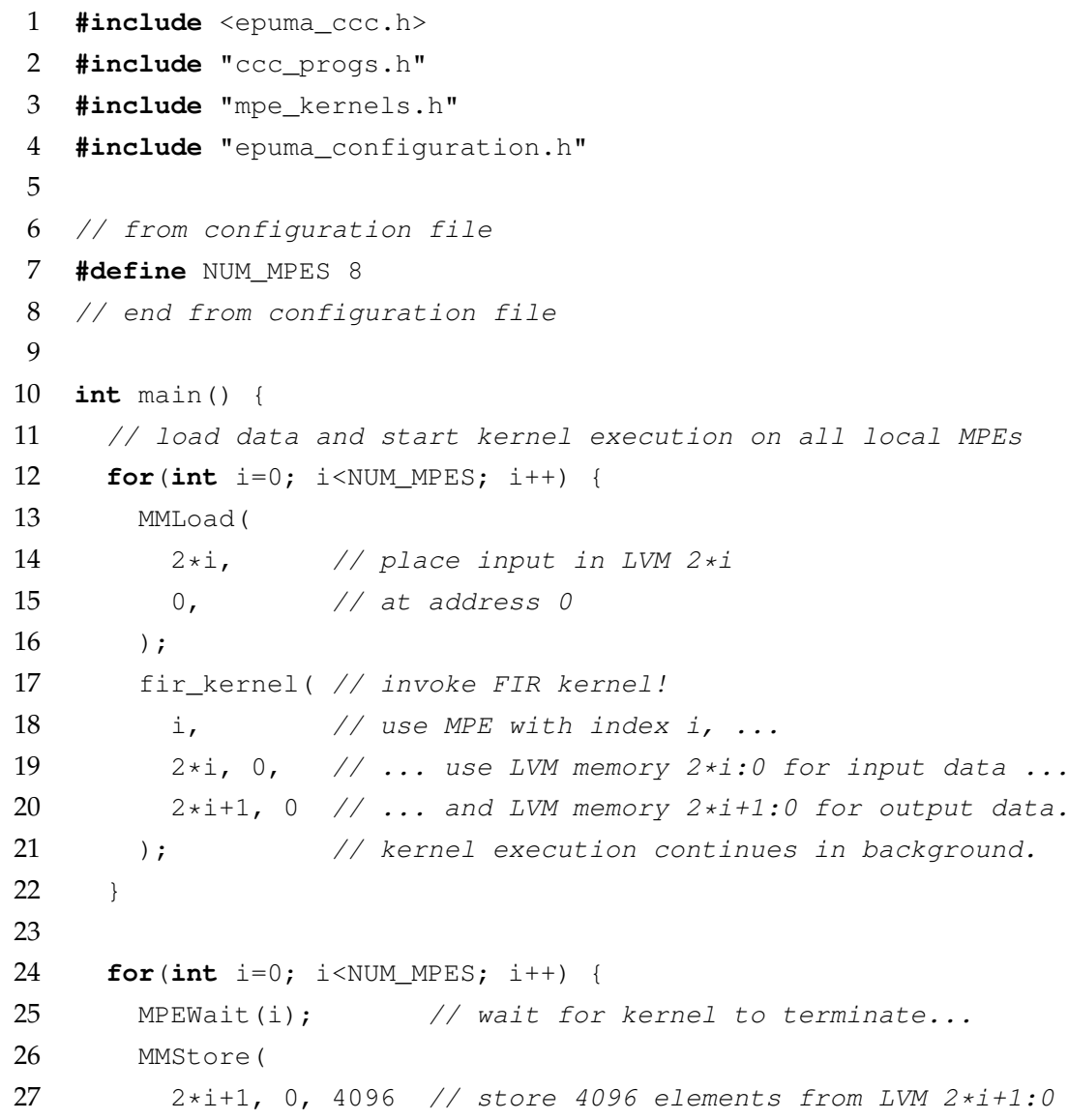


Still, since ePUMA targets applications with extreme efficiency requirements, any high-level methodology may not be appropriate because of high overhead. Reaching an acceptable compromise between programming simplicity and execution time overhead is still a challenge to be faced. Several interesting possibilities have been highlighted and will be addressed in future research. 


\section{Part V}

\section{Evaluation}





\section{Chapter 15}

\section{MPE evaluation}

This chapter will evaluate the ePUMA MPE and the hardware concepts proposed in this thesis. The evaluation will be based on benchmarking of the implemented MPE kernel library, a large library of common DSP kernels that have been optimized for the architecture. The impact of different hardware features will be discussed and the overall results will be compared with other architectures.

\subsection{Methodology}

There are many ways to estimate or evaluate the performance of a DSP processor [4]. The easiest way is possibly to simply look at the stated theoretical number of integer or floating-point operations that the processor can perform per cycle. This gives a hint of the processor's performance, but since real workloads seldom reach $100 \%$ utilization of the processor, the accuracy of such a performance prediction is rather poor. In contrast, measuring the performance of a complete application is a good way of evaluating that particular application, but it is generally hard to extrapolate the performance of other applications based on such data. Additionally, a large application may be very complex and other factors, such as the programmer's skill, may greatly influence the results. Measuring the performance of common compute kernels may be the best trade-off between these two extremes. It represents real workloads common to 
many applications and the complexity of each kernel is not too great, which makes it feasible to cover many applications with a reasonable workload. Since many DSP applications will be dominated by kernel execution time, relatively good application performance approximations can be made from the kernel execution times only, although this is certainly not always the case.

To evaluate an architecture based on kernels, one must of course select which algorithms to implement. Research projects tend to use a small number of representatives, which may or may not reflect the performance of other kernels. Kernels of simple structure are commonly used, e.g. matrix multiplication. Such kernels are simple to implement and will generate optimistic results, but the most interesting results are probably obtained from more complex kernels.

In this research the choice has been to implement as many kernels as possible during the project timeframe. The main purpose is to show that the proposed concepts and architecture do not only work well for a few kernel examples, but that they generally work well within the DSP domain. A problem with this approach is that there are very few other relevant architectures with extensive benchmarking data publically available. One exception is the Texas Instruments (TI) DSP kernel libraries which include many common transforms, matrix operations, math functions, filters and image processing kernels [53, 144, 145]. The same kernels have been implemented for the MPE, which allows a direct comparison between a VLIW-based DSP and a HOF-based DSP. Data for the Freescale SC3850 is also available, but the kernel set is much smaller.

The hardware characteristics of the TI c66x DSP, the Freescale SC3850 and the ePUMA MPE are compared in Table 15.1. The c66x is the most powerful design in the TI DSP family. The c66x and the MPE with a large instruction set configuration have similar capabilities and support similar data types. The c66x additionally supports double-precision floatingpoint operations, but the MPE has more application-specific instructions. The possibly most important difference is that the c66x uses a shared memory cache-based architecture compared to the distributed memory 
Table 15.1: Comparison - Single-core TI c66x, Freescale SC3850 and ePUMA MPE. The power consumption for the SC3850 is estimated from a full system including peripherals $\left({ }^{*}\right)$.

\begin{tabular}{|c|c|c|c|}
\hline & $\begin{array}{c}\text { TI c66x } \\
{[59](40 \mathrm{~nm})}\end{array}$ & $\begin{array}{c}\text { Freescale SC3850 } \\
{[146](45 \mathrm{~nm})}\end{array}$ & $\begin{array}{c}\text { ePUMA MPE } \\
(28 \mathrm{~nm})\end{array}$ \\
\hline Clock freq. & $1.25 \mathrm{GHz}$ & $1 G H z$ & $500 M h z$ \\
\hline 16-bit multipliers & 32 & 8 & 16 \\
\hline Float ops/cyc & 8 & - & 4 \\
\hline General mem. & $1 M B \mathrm{~L} 2$ & $512 k B$ L2 & - \\
\hline Data mem. & - & - & $2 x 64 k B$ \\
\hline Program mem. & - & - & $16 k B$ \\
\hline Memory area & $3.24 \mathrm{~mm}^{2}$ & $?$ & $0.29 \mathrm{~mm}^{2}$ \\
\hline Core area & $3.24 \mathrm{~mm}^{2}$ & $?$ & $0.14 m m^{2}$ \\
\hline Total power & $1180 m W$ & $620 m W^{*}$ & $70 m W$ \\
\hline
\end{tabular}

architecture of ePUMA. The c66x is, at least on paper, a much more powerful DSP than the MPE. The difference can easily be explained by that the sum of all datapaths results in a two times wider datapath for the c66x and a 2.5 times higher clock frequency. On the other hand, the power consumption is much higher. The shared memory system has a high cost in terms of hardware, with approximately half of the core area spent on memory controllers and other memory-supporting functions. Less information is available about the SC3850. The power consumption of a processor core is estimated as follows: A full system including peripherals consumes $2.9 \mathrm{~W}$ in a 1-core configuration and $6 \mathrm{~W}$ in a 6-core configuration [146]. The addition of 5 cores thus adds $3.1 \mathrm{~W}$ of power consumption, or $0.62 \mathrm{~W}$ per core.

In addition to comparing the MPE with other architectures, it is also interesting to compare it to itself. For example, it would be unfair to compare the SIMD concept with the HOF concept using two different architectures, because many other factors would influence the results. However, a HOF-based processor is compatible with SIMD-style execution by 
only issuing instructions that process vectors of length equal to datapath width. In this way the concepts can be compared without implementing another processor. Finally, we can compare actual kernel performance with the theoretical performance limit of a kernel only considering compute work. This indicates how close a kernel is to the computing limit of the architecture and how well computing, data access and control operations have been orthogonalized.

Since the MPE is configurable in terms of hardware features, the evaluation will be affected by e.g. the choice of available processor instructions. In practice, the kernel implementation process has not been made with a constant pre-defined instruction set. Whenever a new kernel has been considered, the potential for hardware modifications have been investigated. In some cases this has meant that a new instruction was mapped to the pre-existing datapath structure. Other features, such as the hardware support for unpredictable memory access that was introduced in Section 8.6, was added as a direct consequence of a few specific kernels. Each additional hardware feature must be evaluated by considering its cost, its potential use, its performance impact and more. Although very specific datapath hardware could have huge benefits in some cases, we've constrained ourselves to only allow new instructions that can be mapped to the datapath structure from Figure 10.4 with only very minor additions.

Another aspect that greatly influences kernel performance is the data distribution over LVM memories and the number of LVMs that can be used during kernel execution. Most kernels accept input data in one LVM memory. The output data may be produced in the same memory as the input data, or in another memory if performance is improved by doing so. However, there are exceptions. Some kernels, such as matrix multiplication, require two input data sets (two matrices) which are best placed in two different memories. Other kernels, such as QR decomposition, only require one input matrix, but produce two, which may or may not be placed in the same memory. By default we use two LVM memories for kernel implementation and distribute the data over these mem- 
ories for best performance. Two LVM memories are enough to avoid a memory bottleneck for most kernels. Note however that an optimal placement of data from a kernel's perspective may not be optimal from the system's perspective when the whole application is considered. It all depends on how the input is produced and how the output data is later used.

Kernel implementation and evaluation is greatly aided by the MPE simulator, a simulator which allows an MPE to be simulated in a standalone environment. The MPE simulator simulates all aspects of the MPE, which means that the cycle times reported by the simulator will exactly match those of real hardware. Since a VLSI-chip has not yet been manufactured, hardware evaluation has primarily been done using an FPGA prototype. As described in Chapter 11, a 28nm MPE layout has been produced and gate-level simulations of the VLSI hardware have been performed to check correctness and estimate energy consumption.

The kernel benchmarking procedure that has been used in this work can be summarized as:

1. Prepare appropriate kernel input data and select kernel parameters.

2. Run the kernel on the MPE simulator and FPGA prototype, with input data available in an LVM memory.

3. Compare simulator and FPGA prototype results. Make sure that the output data and measured cycles times are the same.

4. Perform a gate-level simulation of the kernel on $28 \mathrm{~nm}$ synthesized VLSI hardware, to check final results and estimate the kernel's energy consumption.

This benchmarking procedure ignores how input data ends up in an LVM memory and what happens with the output data. Benchmarks of other DSP architectures typically make the same assumptions, such as a warm cache. If the control flow is predictable and the input/output bandwidth is sufficient, we should be able to come close to these values for real applications as well. 
The remainder of this chapter will be devoted to case studies of different kernel implementations. Data types are denoted as follows: intXX represents XX-bit integer/fixed-point. $\mathbf{u X X}$ and $\mathbf{s X X}$ is used to specifically denote unsigned and signed integer/fixed-point formats. fp32 is used for 32-bit floating-point kernels. To denote the use of complexvalued arithmetic, a c is prepended to any other data type, e.g. cint16 (for 16+16-bit fixed-point) or cfp32 (for complex-valued 32+32-bit floatingpoint).

\subsection{Case study: Basic vector/matrix element- wise operations}

Although conceptually very simple, basic kernels which principally only perform a single operation per input data element, are challenging for most architectures. This is because temporal data locality is poor (every element is only used once) and the memory bandwidth requirements are very high. Many architectures do not even provide enough local memory bandwidth to perform these operations at the performance limit of the datapath. When these types of operations are used at the system level, it may be impossible to use more than one processing core, since the main memory is fully utilized.

The TI c66x DSP kernel library implements a few element-wise kernels that we will use for comparison [53, 145]. Their performance is reported as performance formulas which express the required cycle time as a function of the number of elements to process. All MPE kernels have been implemented in eOctave and the main compute work translates into a single assembly instruction in most cases. For this particular experiment, results for an MPE with 2 respectively 3 LVM memories are included. The results are shown in Table 15.2. Although the c66x is theoretically more powerful, the MPE performs better. This is primarily because the c66x lacks in local memory bandwidth. The c66x uses dual 64-bit data busses, whereas the MPE can retrieve 128-bits from each LVM, which gives the MPE 2 or 3 times more data memory bandwidth depending on 
Table 15.2: Performance of simple element-wise kernels. The formulas represents the number of cycles it takes to process $N$ elements. For the c66x, the number of elements $N$ must typically be a multiple of 4 or 8 , depending on the kernel. The MPE can handle any value of $N$, but the cycle times must be integer and ceiling operations have been omitted for simplicity.

\begin{tabular}{l|ccc}
\hline \hline & \multicolumn{3}{|c}{ Cycle formula [cycs] } \\
Kernel & TI c66x [53, 145] & MPE 2LVM & MPE 3LVM \\
\hline Addition int16 & $3 / 8 \mathrm{~N}+19$ & $2 / 8 \mathrm{~N}+7$ & $1 / 8 \mathrm{~N}+7$ \\
Addition int32 & $3 / 4 \mathrm{~N}+20$ & $2 / 4 \mathrm{~N}+7$ & $1 / 4 \mathrm{~N}+7$ \\
Addition fp32 & $3 / 4 \mathrm{~N}+22$ & $2 / 4 \mathrm{~N}+10$ & $1 / 4 \mathrm{~N}+10$ \\
Multiplication int32 & $3 / 4 \mathrm{~N}+25$ & $2 / 4 \mathrm{~N}+10$ & $1 / 4 \mathrm{~N}+10$ \\
Multiplication fp32 & $3 / 4 \mathrm{~N}+24$ & $2 / 4 \mathrm{~N}+10$ & $1 / 4 \mathrm{~N}+10$ \\
Negation int32 & $1 / 2 \mathrm{~N}+20$ & $1 / 4 \mathrm{~N}+7$ & $1 / 4 \mathrm{~N}+7$ \\
Reciprocal int16 & $8 \mathrm{~N}+44$ & $5 / 4 \mathrm{~N}+34$ & $5 / 4 \mathrm{~N}+34$ \\
Reciprocal fp32 & $3 / 2 \mathrm{~N}+36$ & $3 / 4 \mathrm{~N}+22$ & $3 / 4 \mathrm{~N}+22$ \\
Convert int16 to fp32 & $1 / 2 \mathrm{~N}+34$ & $1 / 4 \mathrm{~N}+7$ & $1 / 4 \mathrm{~N}+7$ \\
Convert fp32 to int16 & $1 / 2 \mathrm{~N}+33$ & $1 / 4 \mathrm{~N}+7$ & $1 / 4 \mathrm{~N}+7$ \\
Clipping int16 & $1 / 4 \mathrm{~N}+32$ & $1 / 8 \mathrm{~N}+11$ & $1 / 8 \mathrm{~N}+11$ \\
\hline \hline
\end{tabular}

configuration. This can be compared with instruction fetching capability: the c66x uses a 256-bit interface to its instruction cache whereas the MPE uses 128-bits to its local store. The ratio instruction-to-data-bandwidth ratio is therefore at least 4 times higher for the VLIW DSP, which clearly indicates that it is much more instruction hungry. The MPE on the other hand, often doesn't even use the local store due to multi-cycle execution, which allows the local store to be used for other purposes or simply idle to reduce energy consumption.

The results in Table 15.2 also show that the constant overhead with each kernel invocation is lower for the MPE than the c66x. The MPE simply issues its compute instruction and it then runs until the entire vector/matrix has been processed. A VLIW processor on the other hand has to setup a software pipeline, which requires several instructions before 
Table 15.3: Code size for the simple element-wise kernels.

\begin{tabular}{l|cc}
\hline \hline & \multicolumn{2}{|c}{ Code size [bytes] } \\
Kernel & TI c66x [53, 145] & MPE \\
\hline Addition int16 & 96 & 8 \\
Addition int32 & 96 & 8 \\
Addition fp32 & 96 & 8 \\
Multiplication int32 & 96 & 8 \\
Multiplication fp32 & 96 & 8 \\
Negation int32 & 96 & 8 \\
Reciprocal int16 & 256 & 40 \\
Reciprocal fp32 & 192 & 16 \\
Convert int16 to fp32 & 128 & 8 \\
Convert fp32 to int16 & 128 & 8 \\
Clipping int16 & 128 & 12 \\
\hline \hline
\end{tabular}

the processing reaches full efficiency. The overhead on the MPE only involves pipeline latency. This overhead is not particularly important for larger data sets, but could represent a significant overhead for smaller data sets. An additional advantage of the MPE kernels is that most kernels for the c66x require that the number of elements $N$ is a multiple of 4 or 8 , depending on the kernel.

Table 15.3 compares the code size of the kernels. The MPE demonstrates an order of magnitude of improvement. This is to be expected, since most of the MPE kernels are single-instruction kernels.

\subsection{Case study: Reductions}

Reduction kernels are not affected by the use of 2 or 3 LVM memories, since the destination is only written once after all memory reads have been performed. The performance formulas and code size of some kernels are given in Table 15.4. Once again, the MPE shows better cycle times and a lower constant overhead than the c66x. However, there is 
Table 15.4: Cycle times and code size of some reduction kernels. The formulas represents the number of cycles it takes to process $N$ elements.

\begin{tabular}{l|cc|cc}
\hline \hline & \multicolumn{2}{|c|}{ Cycle time [cycs] } & \multicolumn{2}{c}{ Code size [bytes] } \\
Kernel & TI c66x & MPE & TI c66x & MPE \\
\hline Block exponent int32 & $1 / 2 \mathrm{~N}+31$ & $1 / 2 \mathrm{~N}+14$ & 224 & 8 \\
Dot product int16 & $1 / 4 \mathrm{~N}+31$ & $1 / 8 \mathrm{~N}+10$ & 96 & 8 \\
Dot product fp32 & $1 / 2 \mathrm{~N}+35$ & $1 / 4 \mathrm{~N}+12$ & 256 & 8 \\
Dot product cfp32 & $1 / 2 \mathrm{~N}+50$ & $1 \mathrm{~N}+11$ & 288 & 8 \\
Dot prod. sq int16 & $1 / 2 \mathrm{~N}+33$ & $1 / 8 \mathrm{~N}+10$ & 128 & 8 \\
Max (index) int16 & $3 / 16 \mathrm{~N}+72$ & $1 / 8 \mathrm{~N}+10$ & 256 & 8 \\
Max (index) fp32 & $3 / 4 \mathrm{~N}+27$ & $1 / 4 \mathrm{~N}+10$ & 192 & 8 \\
Max (value) int16 & $1 / 8 \mathrm{~N}+25$ & $1 / 8 \mathrm{~N}+10$ & 128 & 8 \\
Min (value) int16 & $1 / 8 \mathrm{~N}+28$ & $1 / 8 \mathrm{~N}+10$ & 128 & 8 \\
Min (value) fp32 & $1 / 2 \mathrm{~N}+26$ & $1 / 4 \mathrm{~N}+10$ & 128 & 8 \\
\hline \hline
\end{tabular}

one exception: the complex floating-point dot product kernel. The c66x has two times the number of floating-point units than the MPE and in this particular kernel they are fully utilized which results in better cycle times. Once again, MPE kernels are much smaller than c66x kernels.

\subsection{Case study: Data rearrangement}

Although data rearrangement operations (e.g. matrix transpose) perform no actual compute work, such operations can be useful since subsequent processing steps may be done more efficiently. The need for explicit data rearrangement is very much dependent on the memory addressing flexibility of the processor. Because the MPE provides very flexible memory access, rearrangement can often be avoided. This is not the case for many other architectures.

In case explicit matrix transpose is required, the MPE can implement it as shown in Figure 15.1. We assume that the original matrix is stored as rows of consecutive elements. Permutation tables can be constructed 


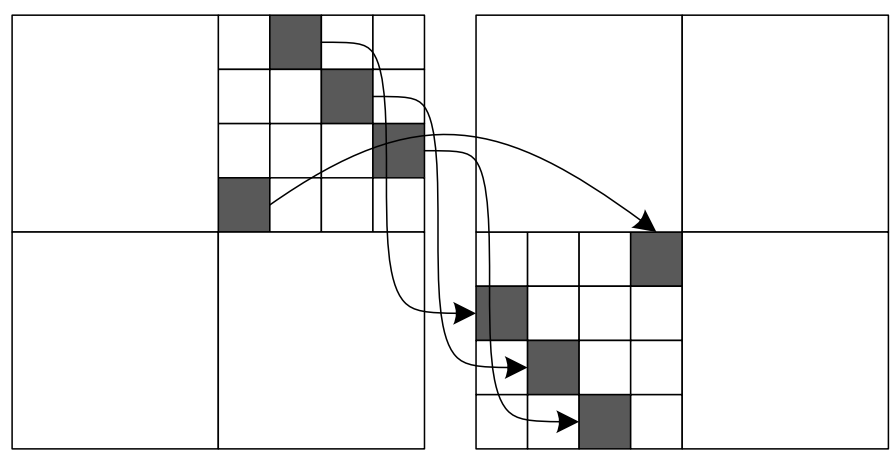

Figure 15.1: Matrix transpose on the MPE. Due to space limitations, 4-way transpose is shown instead of the actual 8-way transpose.

Table 15.5: Performance of matrix transpose for some different architectures.

\begin{tabular}{l|c}
\hline \hline Architecture & Cycles per element \\
\hline Core2 [147] & $0.41-1$ \\
Cell SPU [147] & $1.38-3.75$ \\
Ardbeg [61] & 0.19 \\
TI c66x [53] & 0.66 \\
MPE & 0.16 \\
\hline \hline
\end{tabular}

to perform direct transpose in a single step, although some restrictions apply. The number of cycles required per element is shown in Table 15.5 for some different architectures. The Core2 and the Cell SPU represent two typical examples of architectures which perform register file shuffles for data rearrangement. This approach requires multiple passes over the input data, which reduces performance. The Ardbeg processor reaches performance similar to the MPE. On the other hand, its SIMD vector width of 512 bits is much wider than all other architectures.

The LUT-based addressing of the MPE is suitable for much more complex data rearrangement operations than matrix transpose. The complexity of address generation for some operations, such as interleaving, cause too much run-time overhead if implemented using processor instructions only. By precalculating memory addressing tables, this overhead 
can be reduced or eliminated. It allows streaming data reordering from one input data LVM memory, directly to an output LVM memory at peak memory bandwidth. The drawback is that the size of permutation tables may be big. However, by combining AGU functionality and lookup tables, it may be possible to generate all required memory addresses for a rearrangement operation from a much smaller lookup table.

\subsubsection{QPP interleaving}

The implementation of QPP interleaving/de-interleaving for LTE on the MPE was studied in [75]. The QPP interleaver relates the output index $x$ to input index $\pi(x)$ according to

$$
\pi(x)=\left(f_{1} x+f_{2} x^{2}\right) \bmod L, \quad 0 \leq x<L
$$

where L is the data block size. $f_{1}$ and $f_{2}$ are constant coefficients dependent on $L$. These constants are defined by the LTE standard and have been selected to properly to define a valid permutation [148]. To avoid the multiplications and modulo operation, address generation can be described recursively as follows:

$$
\begin{aligned}
\pi(x+n) & =\left(f_{1} x+f_{2} x^{2}+f_{1} n+f_{2} n^{2}+2 f_{2} n x\right) \bmod L \\
& =\left(\pi(x)+\delta_{n}(x)\right) \bmod L \\
\delta_{n}(x) & =\left(f_{1} n+f_{2} n^{2}+2 f_{2} n x\right) \bmod L
\end{aligned}
$$

The function $\delta_{n}(x)$ can also be recursively generated as

$$
\delta_{n}(x+k)=\left(\delta_{n}(x)+2 f_{2} n k\right) \bmod L
$$

Since $\delta_{n}(x)<L, \pi(x)<L$ and $\left(2 f_{2} n k\right) \bmod L$ is a constant $<L$, the modulo operations can be simplified into subtract-and-compare operations.

Instead of using the above-mentioned formulas to calculate memory addresses, one could simply store the complete data access table for every possible block size. There are 188 possible block sizes ranging from $L=40$ to $L=6144$ elements. This would require a LUT with at least $\sum_{i} L_{i}=355248$ entries, which would require approximately $700 \mathrm{kB}$ of 


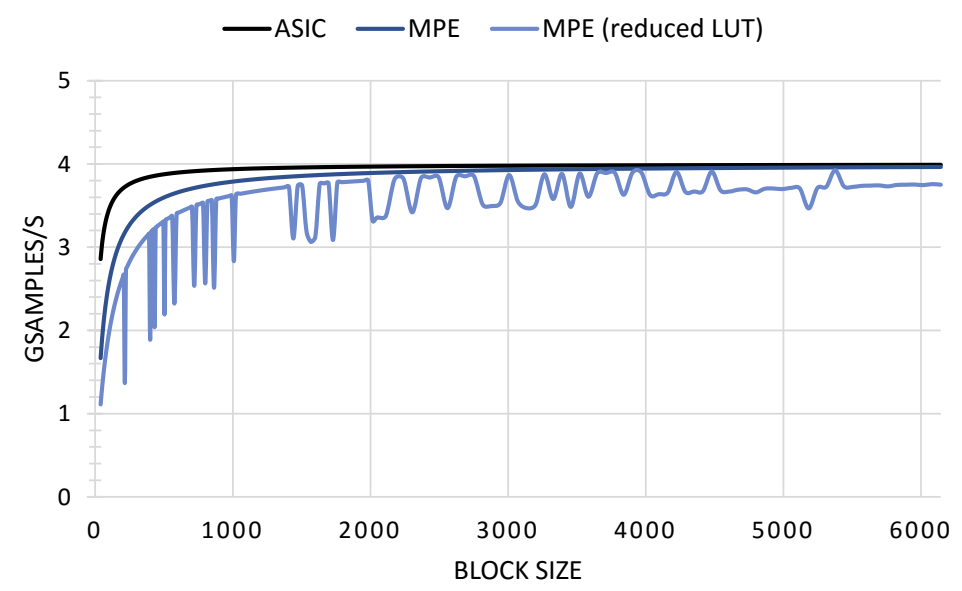

Figure 15.2: QPP interleaving performance of the MPE compared to an 8-way QPP-ASIC.

memory on the MPE, which is not feasible keep on-chip. However, by using subtract-and-compare functionality in the LVM AGU, one can combine AGU functionality with LUTs and compress the required LUTs to roughly $7 \mathrm{kB}$. The details are given in [75].

A QPP-ASIC implementation is given in [36] which we use for comparison. Since our LVM memories support up to 8 parallel memory accesses, the QPP-ASIC is extended to an 8-way ASIC implementation. There is little point in comparing energy efficiency and hardware area of the ASIC and the MPE: the area and energy consumption of the ASIC is in the order of an LVM AGU. The main question is rather whether the MPE can approach the performance of the ASIC or not, without additional QPP-specific hardware.

Figure 15.2 shows the performance of the ASIC compared to the MPE. The main difference is the fact that the MPE has to execute some setup code and the overall pipeline latency is longer. However, the actual interleaving is performed by a single long copy instruction, which processes eight elements per clock cycle. This translates to a rate of 4 GSamples/s 
at 500MHz. It is possible to further reduce the LUT size. In the implementation MPE (LUT reduced), the LUT size has been reduced from $7 \mathrm{kB}$ to $3 \mathrm{kB}$. The cost is additional pre-processing. It is possible to reduce LUT size even further, however it is not possible to completely get rid of LUTs. At the very least, the interleaving constants $f_{1}$ and $f_{2}$ must be tabulated.

The size of the 8-way ASIC interleaving circuit is 75\% larger than an LVM AGU, and can only be used for QPP interleaving. Adding such an interleaving circuit as part of an LVM AGU would princially not improve performance. By using the local store to store LUTs, we reuse the already available hardware and keep software programmability.

\subsection{Case study: Linear filters}

Filtering is one of the most typical DSP processing jobs and linear filters are the most common. The MPE kernel library implements many types of linear filters, including general and symmetrical FIR filters, IIR filters, interpolation/decimation filters and two-dimensional filters for e.g. image processing. In this section we will investigate the impact of the proposed HOF concept in detail, by implementing multiple versions of general FIR-filtering. Similar results can be obtained for most other similar filter types. We will also investigate the energy efficiency of the MPE compared to a similar an FIR ASIC implementation. Finally, we will investigate the implementation of 2D convolution.

\subsubsection{Implementation of a general FIR-filter}

The inner computation of an FIR-filter can be written as

$$
y[n]=\sum_{i=0}^{T-1} c_{i} x[n-i],
$$

which produces a single output sample $y[n] . N$ such computations can be performed to produce $N$ output samples for consecutive values of $n$. The basic data access pattern is very simple. However, in some situations 
the input data may be organized in some non-obvious way, or alternatively, one may want to avoid multiplications with zero, when $c_{i}=0$. These situations can be handled without a performance penalty using permutation vectors, but these situations will not be further discussed.

Multiple versions of general FIR-filtering have been implemented for the MPE. The main purpose is to evaluate different implementation strategies and compare HOF processing to SIMD processing. The implemented kernels can be divided into the three following categories:

- HOF-2D: Kernels which may use the full feature set of the MPE, including 2D block patterns and 2D memory addressing.

- HOF-1D/vector: Kernels which are limited to use only non-block HOF instructions and 1D memory addressing features. This effectively makes the MPE similar to a vector processor.

- SIMD: Kernels which are limited to use only non-block HOF instructions which only process vectors of exactly datapath width and 1D memory addressing. This makes the MPE similar to a SIMD processor.

Note that the SIMD category may be similar to some other SIMD architectures, but still it allows operations directly on memory, unaligned memory access, AGU memory pointer incrementation features and reduction instructions (which technically are not traditional SIMD), features which many other SIMD architectures lack. This means that SIMD in this context is likely to be more flexible and energy-efficient than many other SIMD architectures. This evaluation instead focuses on comparing the multi-dimensional execution style of HOF instructions, with the fixed-size vector execution style of SIMD instructions.

All evaluated kernels implement an FIR filter according to Listing 15.1. The inner for-loop is translated into one or multiple MAC reduction instructions. The list of all implementations is:

- SIMD: Basic: A basic SIMD implementation using MAC reduction. 
Listing 15.1: A simple FIR-filter implementation.

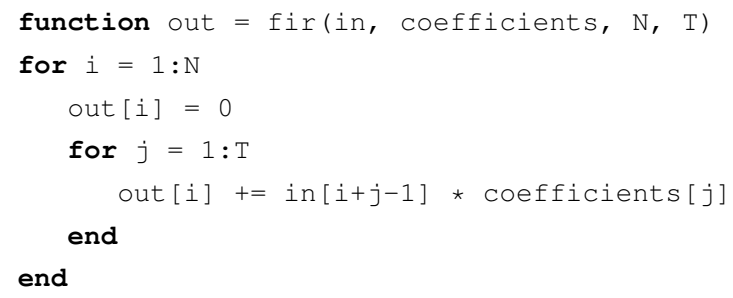

- SIMD: Unroll-inner-2: A basic SIMD implementation with inner loop unrolling, with a loop unrolling factor of 2 .

- SIMD: Unroll-inner-FULL(8): Same as the previous version, but with fully unrolled inner loop.

- SIMD: + Hardware repeat: Same as the previous version, but the outer loop is implemented using hardware repeat rather than conditional jump instructions.

- SIMD: + Unroll-outer-4: Same as the previous version, but the outer loop is also unrolled a factor of 4 , which eliminates some execution time overhead from bookkeeping instructions.

- HOF-1D: Basic: A basic HOF implementation. Compared to the SIMD implementation, the inner loop can be implemented with a single compute instruction plus additional control instructions.

- HOF-1D: + Hardware repeat: HOF-1D version of SIMD: + Hardware repeat.

- HOF-1D: + Unroll-outer-4: HOF-1D version of SIMD: + Unrollouter-4.

- HOF-2D: The entire compute operation can be expressed with a single compute instruction and some additional setup code.

All implementations assume that the input data is read from one LVM memory and the output is stored in another memory. The filter coeffi- 
cients are fixed and part of the kernel program in this case, which means that they are originally stored in the local store. Two alternatives are investigated: either the filter coefficients remain in the local store, or alternatively, the kernel starts by copying the coefficients to the register file. All kernels use complex-valued fixed-point data, 16 bits for the real and imaginary parts respectively. The filter size is set to $N=4096$ and $T=32$. The value of $N$ is rather insignificant, but the value of $T$ (the number of coefficients) is selected so that the filter coefficients can fit in the MPE register file.

The results are shown in Table 15.6. The theoretical lower bound on execution time counting only compute work is $t_{\geq c y c}=N \cdot T / P$. The MPE has a processing width $P=4$ for $16+16$-bit complex data, which results in $t_{\geq c y c}=4096 \cdot 32 / 4=32768$ clock cycles. The table indicates the percentage of this peak performance achieved. Additionally, normalized energy, code size and the number of instruction vectors fetched from the local store are shown ${ }^{1}$.

The best SIMD-based implementation reaches $82 \%$ of peak performance, with the filter coefficients stored in the register file. By keeping the coefficients in the local store, performance is lower, because filter coefficient fetching and instruction fetching compete for the local store, since the constant data cache is not large enough to hold all constants. If the constant data cache size is increased, this implementation can reach the same level of performance as the register file-based approach. It is possible to improve the performance of the register file-based approach further, by using more loop unrolling, but only at a significant cost in code size.

The best HOF-1D implementation also reach $82 \%$ of peak performance. This is simply because the only difference between the best SIMD implementation and the best HOF-1D implementation is that the inner loop

\footnotetext{
${ }^{1}$ Instruction vector fetches are the actual number of 128-bit vectors fetched from the local store by the instruction fetching hardware. Each 128-bit vector may hold one or multiple instructions, thus the value does not exactly correspond to the number of executed instructions. Additionally, it includes speculative buffering of instructions, instructions which may be discarded due to non-linear control flow.
} 
Table 15.6: Evaluation of general FIR-filter implementations on the ePUMA MPE. The best result in each category is underlined.

\begin{tabular}{|c|c|c|c|c|}
\hline Implementation & 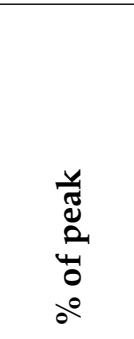 & 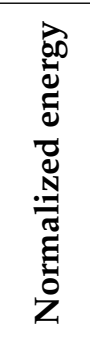 & 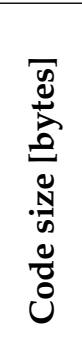 & 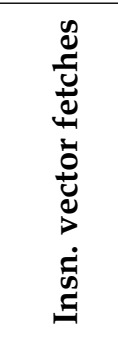 \\
\hline \multicolumn{5}{|c|}{ Coefficients in local store } \\
\hline SIMD: Basic & $9.5 \%$ & 5.23 & 128 & 131075 \\
\hline SIMD: Unroll-inner-2 & $17.8 \%$ & 3.64 & 144 & 77827 \\
\hline SIMD: Unroll-inner-FULL(8) & $38.1 \%$ & 2.35 & 160 & 32773 \\
\hline SIMD: + Hardware repeat & $44.4 \%$ & 2.09 & 128 & 24581 \\
\hline SIMD: + Unroll-outer-4 & $47.1 \%$ & 2.05 & 400 & 23557 \\
\hline HOF-1D: Basic & $44.4 \%$ & 2.06 & 96 & 20484 \\
\hline HOF-1D: + Hardware repeat & $53.3 \%$ & 1.80 & 80 & 12293 \\
\hline HOF-1D: + Unroll-outer-4 & $60.4 \%$ & 1.71 & 176 & 9221 \\
\hline HOF-2D & $99.93 \%$ & 1.41 & $\underline{64}$ & $\underline{7}$ \\
\hline \multicolumn{5}{|c|}{ Coefficients in register file } \\
\hline SIMD: Basic & $9.5 \%$ & 4.82 & 192 & 131079 \\
\hline SIMD: Unroll-2 & $17.8 \%$ & 3.33 & 208 & 77831 \\
\hline SIMD: Unroll-inner-FULL(8) & $50.0 \%$ & 2.00 & 224 & 36872 \\
\hline SIMD: + Hardware repeat & $66.6 \%$ & 1.56 & 192 & 20490 \\
\hline SIMD: + Unroll-outer-4 & $82.0 \%$ & 1.53 & 432 & 20490 \\
\hline HOF-1D: Basic & $44.4 \%$ & 1.72 & 112 & 24580 \\
\hline HOF-1D: + Hardware repeat & $66.6 \%$ & 1.28 & 96 & 8199 \\
\hline + Unroll-outer-4 & $82.0 \%$ & 1.21 & 160 & 6151 \\
\hline HOF-2D & $99.91 \%$ & $\underline{1.00}$ & 80 & 8 \\
\hline
\end{tabular}

compute instructions can be written as one single vector instruction instead of several SIMD-like instructions. The only practical difference this makes is that the code size is decreased and energy efficiency is improved 
Table 15.7: Hardware configurations for the MPE and an FIR-ASIC.

\begin{tabular}{l|cc}
\hline \hline Component & MPE & ASIC \\
\hline Input sample memory & $64 \mathrm{kB}$ & $64 \mathrm{kB}$ \\
Output sample memory & $64 \mathrm{kB}$ & $64 \mathrm{kB}$ \\
Coefficient storage & 32-entry RF & 32-entry RF \\
Local store & $16 \mathrm{kB}$ & None \\
Datapath & Full MPE datapath & MAC-reduction tree \\
AGU & Full MPE AGU & FIR-specific AGU \\
Performance & 4 samples/cyc & 4 samples/cyc \\
\hline \hline
\end{tabular}

because of fewer instruction fetches.

The two HOF-2D implementations reach $99.93 \%$ and $99.91 \%$ of the architectural performance limit. The main work of the FIR processing is performed by a single compute instruction. Since the local store is principally unused for instruction fetches, it can be used freely to provide filter coefficients. This is also the reason why the local store approach is slightly faster in this case, since it takes 8 additional cycles to copy the filter coefficients from the local store to the register file in case of the register file-based approach. The local store-based approach is best in terms of code size as well, but in practice the most important difference between these two approaches is the fact that the register file-based approach is noticeably more energy-efficient. This is simply because register file read operations are more energy-efficient than memory read operations in the MPE.

\subsubsection{Comparison with an FIR-filtering ASIC}

The HOF-2D solutions just presented consume extremely little energy on instruction fetching and these solutions should therefore be able to approach the energy efficiency of an FIR-ASIC solution. This section will investigate the matter.

Table 15.7 shows hardware setups for the MPE and an FIR-ASIC with 
Table 15.8: The difference in energy consumption between the MPE and an FIRASIC implementation.

\begin{tabular}{l|cc}
\hline \hline & \multicolumn{2}{|c}{ Relative energy [\%] } \\
Unit & MPE & ASIC \\
\hline Data memory & $58 \%$ & $58 \%$ \\
Local store & $0 \%$ & - \\
Other data access & $7 \%$ & $6 \%$ \\
Datapath & $31 \%$ & $22 \%$ \\
Other & $3 \%$ & $1 \%$ \\
\hline Total & $\mathbf{1 0 0} \%$ & $\mathbf{8 8 \%}$ \\
\hline \hline
\end{tabular}

input- and output sample memories. Both implementations perform MAC-reduction, 4 complex values at a time. Both designs support an arbitrary programmable number of input samples and coefficients, limited only by the available memory. The input samples are stored in $64 \mathrm{kB}$ memories and the coefficients are stored in a 32-entry register file (for a maximum of 32 coefficients). We use a single MPE in a 2-LVM setup and an otherwise full default configuration. The ASIC uses an applicationspecific 4-way MAC reduction tree datapath and FIR-specific AGUs. The FIR-ASIC lacks a local store, since it doesn't use an instruction flow and is designed for a single function only.

Table 15.8 compares the relative energy consumption of the MPE with the FIR-ASIC. The sample memories consume the most energy and since these are the same for both designs, the energy consumption is also the same. The ASIC solution consumes no local store energy since it doesn't have one, but the MPE local store consumes very little energy as well, due to the very low local store activity. The main difference between the two implementations is the datapath energy consumption. The data has to pass several more layers of multiplexing in the MPE datapath compared to the ASIC, which is one of the most important reasons for the higher energy consumption. It is likely possible to energy-optimize the MPE datapath further, but some level of energy overhead is to be 
expected. Overall, the complete ASIC with sample memories only reduces energy consumption by $12 \%$ compared to the MPE. The best result known to us for another processor architecture is the ELM architecture, which consumes $50 \%$ additional energy compared to an ASIC solution [29]. The MPE design clearly has less energy overhead than this.

The implemented ASIC is certainly not the most energy-efficient implementation. It is instead intended to similar to the MPE in terms of FIRprogrammability and performance, to quantify the energy overheads of a generalized datapath and the programmability of the MPE. For example, the input samples could be kept in an input sample shift register instead of memory and the datapath could be increased to a 32-way datapath, to generate one output sample per clock cycle. Also, if we have more information about filter structure we can apply many additional optimizations to improve its performance and energy efficiency. However, if filtering is critical to some application, similar modifications could be done to the MPE as well, which would let the MPE follow the ASIC in terms of energy efficiency.

\subsubsection{D convolution}

Convolution in two dimensions is commonly used to perform linear filtering on 2D objects such as images [40]. The operation is similar to FIRfiltering or 1D convolution, but the coefficients are instead organized in a two-dimensional $n \times n$ mask. If the object to filter is an image, each pixel in the resulting image will be a weighted sum of an $n \times n$ neighborhood defined by the filtering mask, as shown in Figure 15.3. Each output pixel will require $n^{2}$ multiplications and $n^{2}-1$ additions for its production.

The complexity of many practical filter implementations can be reduced. If the filter mask can be written as the convolution of two onedimensional masks, one row-mask and one column-mask, the computational complexity is reduced from $n^{2}$ multiplications to $2 n$ multiplications. The commonly used (horizontal) Sobel kernel can be separated 


\begin{tabular}{|c|c|c|c|}
\hline$x_{0}$ & $x_{1}$ & $x_{2}$ & $x_{3}$ \\
\hline$x_{W}$ & $\underline{x}_{w+1}$ & $\underline{x}_{w+2}$ & $\underline{\underline{x}} \underline{w+3}$ \\
\hline$x_{2 W}$ & $\underline{x}_{2 W+1}$ & $\underline{x_{2 W+2}}$ & $\underline{X}_{2 W+3}$ \\
\hline$x_{3 W}$ & $\underline{x}_{3 W+1}$ & $\underline{X}_{3 W+2}$ & $\underline{x}_{3 w+3}$ \\
\hline
\end{tabular}

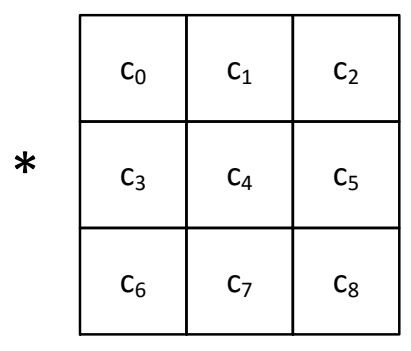

$\mathrm{y}_{2 \mathrm{~W}+2}=\mathrm{x}_{\mathrm{w}+1} \mathrm{C}_{0}+\mathrm{x}_{\mathrm{W}+2} \mathrm{C}_{1+} \mathrm{x}_{\mathrm{W}+3} \mathrm{C}_{2+}$

$\mathrm{X}_{2 \mathrm{~W}+1} \mathrm{C}_{3+} \mathrm{X}_{2 \mathrm{~W}+2} \mathrm{C}_{4+} \mathrm{X}_{2 \mathrm{~W}+3} \mathrm{C}_{5+}$

$\mathrm{X}_{3 \mathrm{~W}+1} \mathrm{C}_{6}+\mathrm{x}_{3 \mathrm{~W}+2} \mathrm{C}_{7}+\mathrm{x}_{3 \mathrm{~W}+3} \mathrm{C}_{8}$

Figure 15.3: 2D convolution.

as

$$
\left[\begin{array}{lll}
1 & 0 & -1 \\
2 & 0 & -2 \\
1 & 0 & -1
\end{array}\right]=\left[\begin{array}{l}
1 \\
2 \\
1
\end{array}\right] *\left[\begin{array}{lll}
-1 & 0 & 1
\end{array}\right]
$$

The row-mask and column-mask will each contain $n$ elements and the filtering of the image can performed by first filtering all image rows using the row-mask, then followed by all columns or vice versa. Multiplications with 0 can also be eliminated if desired.

2D convolution is easily implemented in the MPE using parallel MAC reduction. Pixels offsets can be stored in a lookup-table. Each output pixel can be computed in $n^{2} / 8$ cycles for a general filter kernel and $2 n / 8$ cycles for a separable kernel (16-bit processing). Processing can be accelerated further, as the standard real-valued MAC-operation only uses 8 of the 16 available multipliers. For example, when loading a row/column of 8 consecutive pixels, we can exploit the overlap between the filtering operations to perform even more work.

Table 15.9 shows the performance achieved as cycles per pixel. The performance of the c66x is included for comparison. For the MPE we include both the results for general convolution kernels, as well as separable kernels. The potential benefits of adding special instructions is also 
Table 15.9: Compute performance for $2 \mathrm{D}$ convolution (16-bit data). The performance is shown as cycles per pixel, excluding some constant overhead which is $<64$ for the $c 66 x$ and $<34$ for the MPE.

\begin{tabular}{l|cccc}
\hline \hline & \multicolumn{4}{|c}{ Kernel size } \\
Architecture & $3 \times 3$ & $5 \times 5$ & $7 \times 7$ & $11 \times 11$ \\
\hline TI c66x (General) [145] & 2.0 & 5.5 & 13.25 & 39.0 \\
MPE (General, MAC) & 1.125 & 3.125 & 6.125 & 15.125 \\
MPE (General, special insn.) & 0.6 & 1.67 & 3.5 & - \\
MPE (Separable, MAC) & 0.75 & 1.25 & 1.75 & 2.75 \\
MPE (Separable, special insn.) & 0.4 & 0.67 & 1.0 & - \\
\hline \hline
\end{tabular}

investigated, instructions which reutilize the already available multipliers and arithmetic units for a very small additional hardware cost. Using special instructions, the MPE can outperform the c66x even in absolute time, despite the lower clock frequency. For the normal MAC implementations, the MPE performs 8 multiplications/cycle, which is increased to 15 multiplications / cycle for $3 \times 3$ and $5 \times 5$ kernels and 14 multiplication$\mathrm{s} /$ cycle for $7 \times 7$ kernels using application-specific instructions. The c66x performs an average of 3.7-4.5 multiplications/cycle, despite having 32 multipliers.

One issue with a very fast kernel-level implementation is that main memory bandwidth may limit compute performance, especially if we consider multi-core execution. Using a general $3 \times 3$ convolution kernel, special instructions and 16-bit pixels, a full ePUMA system would be limited by main memory beyond 2 MPE cores, considering a main memory bandwidth of 128 bits/cycle. If we instead use 8-bit pixels, we double the pixel rate over the main memory interface. The existing kernels and instructions can be reutilized by converting the pixels to 16 bits during processing. The data type conversions and final truncation to 8 bit data (including saturation) adds 0.25 cycles of processing per pixel. This allows the use of up to $6 \mathrm{MPE}$ cores. With an image size of $1920 \times 1080$ pixels and an ePUMA system running at 500MHz, $6 \mathrm{MPE}$ cores can filter 
images at a rate of approximately 1700 images per second. Unused MPE cores could potentially be power-gated to reduce energy consumption.

\subsection{Case study: Discrete Fourier Transform}

The Fourier transform is another example of an extremely important DSP kernel. In this section we shall investigate the implementation of Fast Fourier Transform (FFT) for 16+16-bit fixed-point complex data. The MPE implementations for other data types will be omitted, as there is less data to compare with. We start by considering the implementation of $2^{x}$-FFTs and then continue with other mixed-radix FFTs.

The implementation of $2^{x}$-FFTs for the MPE is a straight-forward process. This topic was already discussed in Section 7.6.3. The datapath contains both a dual complex radix-2 butterfly instruction and a single complex radix-4 butterfly instruction. The latter was shown in Figure 10.1. Both instructions include pre-multiplication with twiddle factors, which means that twiddle factor multiplication does not consume extra cycles.

Each FFT layer can be implemented with a single vector butterfly instruction. Input data and twiddle factors are typically placed in an LVM and the local store respectively. Depending on the size of the local store and the size of the FFT, one may alternatively place twiddle factors in an additional LVM memory. Two LVM memories act as a ping-pong buffer during processing. Input/output data addressing is achieved using at least one unique permutation vector per processing layer. However, due to the low permutation vector fetching requirements, the local store is virtually free to fetch twiddle factors without conflicts.

The benchmark results for some different architectures are shown in Table 15.10. These performance values assume that input data and twiddle factors are already loaded to local memory. The MPE butterfly instructions allow the MPE to reach better cycle times than all commercial competitors. In addition, the code is much less complex compared to the fine-grained VLIW implementations. The BBP2 is an application-specific 
Table 15.10: Cycle times for 16-bit complex-valued fixed-point $2^{x}$-FFT.

\begin{tabular}{l|ccccc}
\hline \hline & \multicolumn{5}{|c}{ Number of points } \\
Architecture & $\mathbf{2 5 6}$ & $\mathbf{5 1 2}$ & $\mathbf{1 0 2 4}$ & $\mathbf{2 0 4 8}$ & $\mathbf{4 0 9 6}$ \\
\hline ADSP-TS201S [149] & 585 & & & & \\
SandBlaster SB3500 [150] & 445 & & 2198 & 4736 & \\
TI c66x [53] & 743 & & & & \\
StarCore SC3850 [151] & 777 & 2179 & 3759 & 10227 & 17973 \\
BBP2 [9] & & & & & 6186 \\
MPE & 306 & 700 & 1340 & 3142 & 6214 \\
\hline \hline
\end{tabular}

processor for baseband processing, which has been implemented at our research group. This design has similar datapath capability to the MPE but also additional optimizations towards FFT such as FFT-specific addressing features and a shorter pipeline. This allows it to reach slightly better performance than the MPE. This is the cost the MPE pays to maintain generality, e.g. by instead using programmable addressing through LUT-based addressing.

\subsubsection{Non-power-of-2 transforms}

Modern mobile communication standards use other factors than 2 for DFT computation, including LTE which uses factors 2, 3 and 5. The structures for 3- and 5-point DFT are more complex than 2- and 4-point DFT, because they are not multiplication-free. A popular structure is the Winograd structure [152]. Its structure is simple to implement in custom hardware, but the structure is highly irregular, at least compared to typical processor datapaths. It is possible to merge 2, 3, 4 and 5-point DFT structures to a unified datapath [153] which reduces total hardware cost, but it is still not directly suitable for implementation in a general processor datapath if existing hardware should be reutilized. Others have used this structure for processor-based implementations, but the target is then an FFT-specific processor [154]. For a more general datapath, there are 
many additional constraints.

In case of 16-bit complex-valued FFT processing, the MPE becomes a 4-way complex-valued processor. The mapping of 3- and 5-point DFT to a 4-way datapath is non-trivial. The 5-point DFT will not even receive all input values in a single clock cycle, which means that it has to be executed over multiple cycles. Since all output values from the DFT depend on all input values and the output values also need 2 cycles to be written to memory, we can easily deduce that an isolated 5-point DFT will require at least 3 clock cycles to execute.

The mapping of 3- and 5-point butterflies to a 4-way datapath is discussed by the author of this work in [155]. The proposed butterflies map well to our generalized datapath structure and can reuse the hardware of our radix-4 butterfly implementation. The signal flow graphs (SFG) for the 3- and 5-point DFTs are shown in Figure 15.4(a) and Figure 15.4(b). The required constants are:

$$
\begin{array}{ll}
C_{31}=\cos (-2 \pi / 3)-1 & C_{32}=\sin (-2 \pi / 3) \\
C_{51}=\cos (-2 \pi / 5)-1 & C_{52}=\cos (-4 \pi / 5)-1 \\
C_{53}=\sin (-2 \pi / 5) & C_{54}=\sin (-4 \pi / 5)
\end{array}
$$

Note that the radix- 5 butterfly cannot be implemented as a single instruction. The dotted lines represents how the implementation can be split into three different instructions so that it fits a 4-way datapath and so that it doesn't require any additional arithmetic units besides the ones already present in the MPE datapath. One important difference between the 3point and 5-point DFT is that there is not enough arithmetic units available to include twiddle factor multiplication in the 3-point implementation. This is however included in the 5-point implementation, which is shown as multiplications with factors $W_{1}, \ldots, W_{5}$. For the 3-point DFT, twiddle factor pre-multiplication must be performed using a separate normal complex multiplication instruction whenever required.

The cycle times for some sizes of FFT are shown in Table 15.11. The cycle times for the SandBlaster and the StarCore are estimated from diagrams, since the exact cycle times are not reported. The cycle times are 


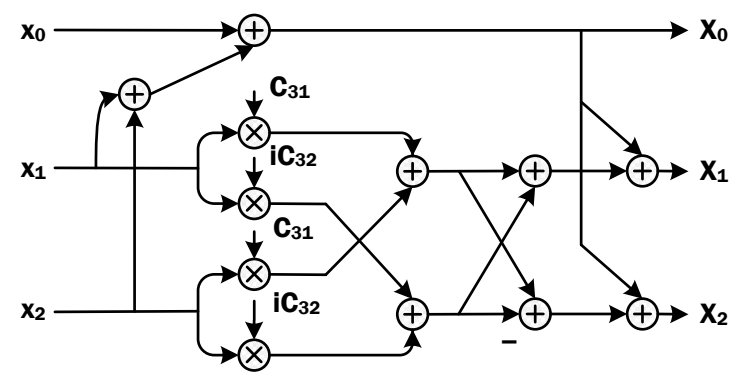

(a) 3-point SFG.

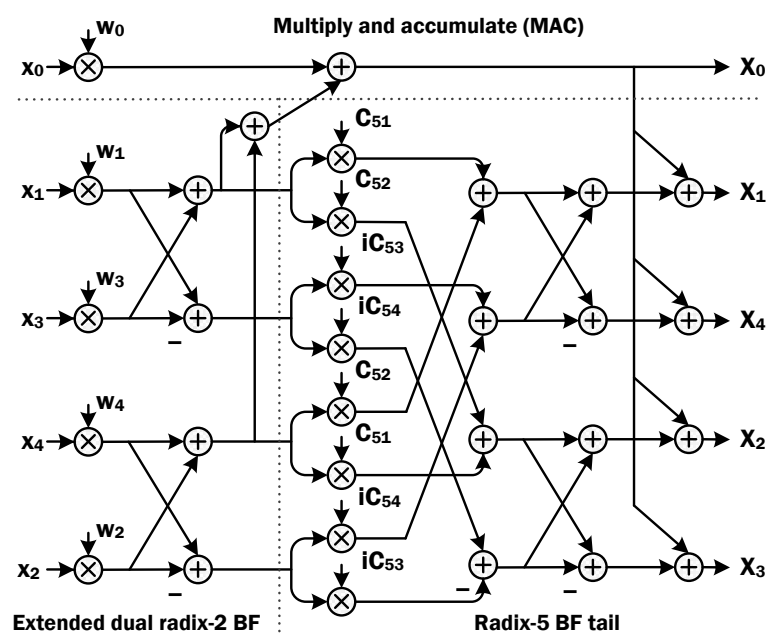

(b) 5-point SFG.

Figure 15.4: Signal flow graphs (SFG) for 3- and 5-point DFT implementations on the MPE.

very much affected by how efficiently each of the 3- and 5-point DFTs have been implemented on the architectures and how many times each butterfly type must be performed. As an example, the 480-point FFT runs faster than the 324-point FFT on the MPE simply because it contains more factors of 2, which results in more use of the faster radix-4 butterfly. 
Table 15.11: Cycle times for 16-bit complex-valued fixed-point mixed-radix FFT.

\begin{tabular}{l|cccccc}
\hline \hline & \multicolumn{6}{|c}{ Number of points } \\
Architecture & $\mathbf{3 2 4}$ & $\mathbf{4 8 0}$ & $\mathbf{6 0 0}$ & $\mathbf{7 6 8}$ & $\mathbf{1 0 8 0}$ & $\mathbf{1 2 0 0}$ \\
\hline SandBlaster SB3500 [150] & 1700 & 3100 & 4200 & 2700 & 5200 & 6700 \\
StarCore SC3850 [156] & 3500 & 3900 & 5700 & 4700 & 11300 & 11600 \\
MPE & 897 & 868 & 1280 & 1070 & 3058 & 2500 \\
\hline \hline
\end{tabular}

Listing 15.2: Histogram generation pseudocode.

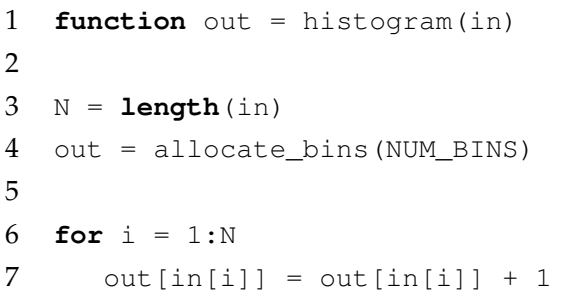

\subsection{Case study: Histogram generation}

Histogram generation represents a kernel that performs unpredictable memory access. It counts the number of occurrences of each possible value or a range of values. It can be used for many different types of statistical operations, such as histogram equalization [40].

Pseudocode for histogram generation is shown in Listing 15.2. If the input is 8-bit image data, there will be $N U M_{-} B I N S=2^{8}=256$ accumulation bins. Data access to the array out is dependent on the input data in array in and the access is a read-modify-write access. Since out is relatively large, it cannot be stored in the register file and must instead be stored in LVM memory. If the main loop is SIMDized by a single HOF instruction, there may be bank conflicts but also possible race conditions, as discussed in Section 11.5. If only an approximate histogram is necessary, one may ignore this issue and accept that there may be errors. However, we shall now only consider an exact histogram calculation. 


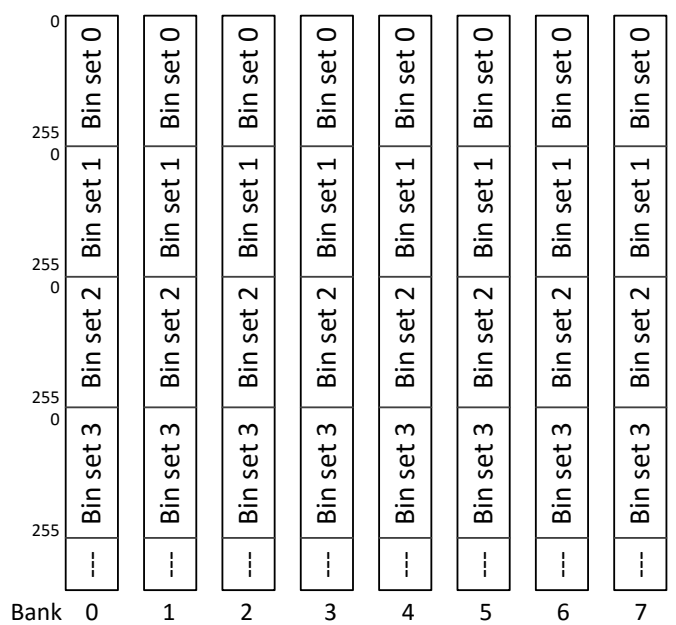

Figure 15.5: Histogram bin distribution over an LVM memory.

To guarantee correct histogram generation, the MPE implementation uses separate bins for each processing lane, as provided by the special addressing features for unpredictable access described in Section 8.6. However, this is not enough to avoid race conditions. Even though each lane keeps its own private bin, two consecutive histogram updates in the same lane may want to modify the same bin. Pipeline latency will determine whether a previous update has had time to be committed to memory or not. As discussed in Section 11.5, the MPE employs a simplified form of stall logic for MPE LVM which resolves this issue. If a memory read operation is initiated and write operations are already in progress, the processor will stall until the write operations are fully committed. This ensures the expected behavior, but for this particular kernel it leads to unsatisfactory performance.

To solve this issue, one may introduce multiple bin sets per processing lane, as shown in Figure 15.5. We start the kernel with the weak memory consistency mode, which essentially disables the LVM stall logic. We introduce enough bins to hide pipeline latency and rotate the use of the bin sets so that no collisions occur. By doing so we use considerably more 
local memory. On the other hand we can guarantee completely conflictfree memory access and data independent execution time. During the processing of a complete image, the bins may be kept over multiple kernel executions and only combined in a single final processing step.

The MPE histogram implementation has been compared to implementations on a GeForce 580GTX and the TI c66x. We compare a single MPE with a single core c66x and the complete GPU. We use only a single MPE and c66x since this is supposed to be kernel-level benchmark and at the same time we avoid potential system-level overheads in this analysis. By only using single cores, main memory bandwidth and communication overheads should not be bottlenecks in the implementations.

Since we don't have access to the other platforms, their energy consumption is estimated. The c66x power estimate of $1180 \mathrm{~mW}$ from [59] is used and the performance estimation is based on the stated number of clock cycles per pixel according to [145]. In practice there will be additional overheads. The GPU has a maximum power consumption of 244W [157]. We use a value of $170 \mathrm{~W}$ for our comparison, which guesses the actual power consumption to $70 \%$ of maximum. Note however that this includes the complete GPU card, including off-chip memories and peripherals. The performance data for the GPU is gathered from [158]. We use the same resolution of 2560x1920 as proposed in this work. The performance of the GPU is an average over multiple images. The execution time on the MPE on the other hand is data oblivious.

The results are shown in Table 15.12. The GPU is by far the fastest, which is not surprising. For the MPE, the performance using both the sequential memory consistency mode and the weaker memory consistency mode is shown, which clearly shows the benefits of using weaker memory consistency in this particular case. When using weak consistency, the MPE is twice as fast as the c66x, despite the lower clock frequency. The code size is relatively large for the MPE in this case. This is because multi-cycle HOF instructions are not applicable and the histogram generation loop must be unrolled. Still, the c66x' code is more than 3 times larger. In terms of energy efficiency, the MPE is superior by far. 
Table 15.12: Benchmarking results for grayscale image histogram generation. The image resolution is $2560 \times 1920$.

\begin{tabular}{|c|c|c|c|}
\hline Architecture & 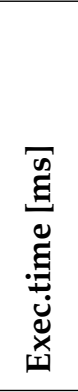 & 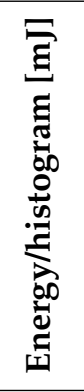 & 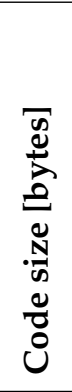 \\
\hline NVIDIA 580GTX & 0.13 & 22.1 & - \\
\hline TI c66x & 4.10 & 3.28 & 608 \\
\hline MPE (seq. cons.) & 8.21 & 0.23 & 192 \\
\hline MPE (weak cons.) & 2.06 & 0.11 & 192 \\
\hline
\end{tabular}

\subsection{Case study: Sorting and non-linear filters}

Sorting is a very common benchmark, even though it is perhaps not the most common DSP kernel. However, it has applications in the DSP domain as well, such as non-linear filtering. This section will investigate how sorting operations can be accelerated on the MPE. We will only consider data sets that can be fit in an LVM memory. Chapter 16 will discuss large-scale sorting of data in off-chip memory and a complete multi-core ePUMA platform. The local sorting procedure introduced here will act as an important building block in the large-scale implementation. In addition we will investigate the implementation of a common non-linear filter: the median filter.

The implementation of sorting on the MPE is based on the observation that the MPE datapath structure can be used to implement small sorting networks [97], as already shown in Figure 10.2(b). Repeated use of such instructions can be used to implement larger sorting networks in software or to implement merge sort [6]. The arithmetic unit network in the MPE datapath primarily supports integer arithmetic. However, the 


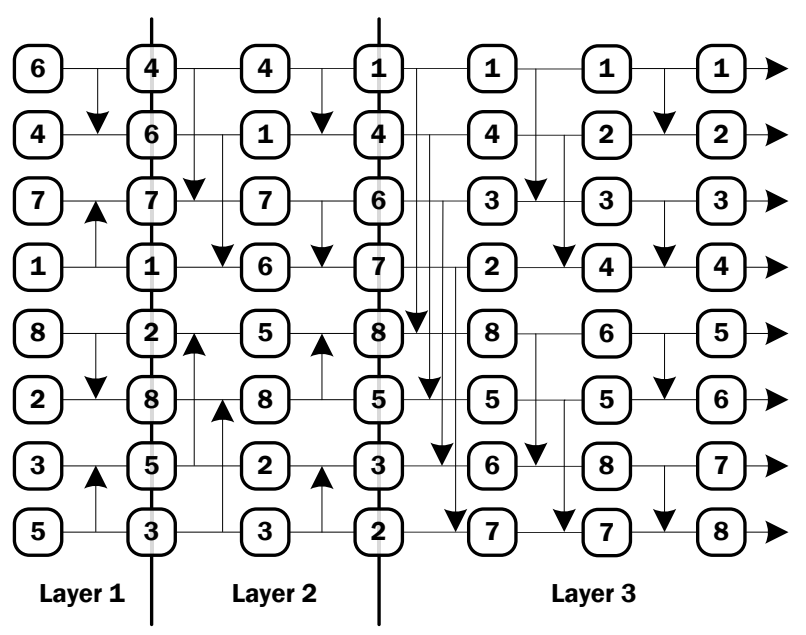

Figure 15.6: An 8-input bitonic sorting network.

IEEE-754 floating-point format lets us easily compare two floating-point values with integer arithmetic, if some minor additional logic is added. By doing so, we can sort both integers and floating-point values on the datapath with the same performance.

Bitonic sorting networks are commonly implemented to accelerate sorting on SIMD architectures [159]. Such a network is shown in Figure 15.6. Sorting networks have a fixed structure of comparators, which is suitable for hardware implementation, but also the innermost critical code in software implementations because no control code is required to determine which comparisons to perform. Furthermore, processing can be performed in blocks of SIMD width to avoid unaligned memory accesses, which would otherwise degrade performance on some SIMD architectures. However, the computational complexity is proportional to $\mathcal{O}\left(N \cdot \log _{2}(N)^{2}\right)$, which is worse than the average-case complexity of $\mathcal{O}\left(N \cdot \log _{2}(N)\right)$ for merge sort. This is the main reason why sorting networks are typically only applied to small-scale sorting in the fastest sorting implementations.

To implement a sorting network on a typical SIMD processor, one typically uses SIMD minimum/maximum instructions to do compar- 


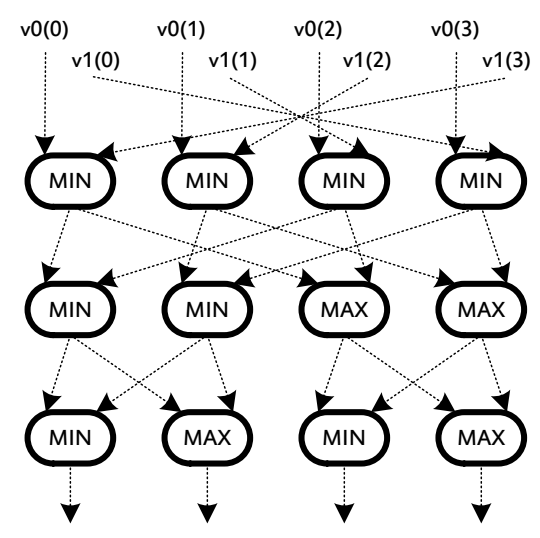

Figure 15.7: A bitonic merge instruction (minmerge) on the MPE datapath.

isons, and shuffle instructions to rearrange data in between comparison layers. One such implementation is given for the x86 in [159], where $6 \mathrm{~min} / \mathrm{max}$ instructions and 7 shuffle instructions are used to implement an 8-way sorting network for 32-bit data. This is equivalent to $13 / 8=1.625$ cycles per element, ignoring other overheads. The MPE equipped with sorting instructions can do the same work in 4 cycles or $4 / 8=0.5$ cycles per element.

Merge sort and bitonic sort both work by repeatedly merging sorted subsequences of $L$ elements into sorted subsequences of length $2 L$, until the whole array of elements is sorted. Merge sort typically works by comparing two elements pointed to by two individual pointers, one for each incoming sorted sublist, and determines which element is smallest and thus is next in line to be put in the sorted output. However, it is possible to compare more than two elements at the same time. Figure 15.7 shows the implementation of an MPE instruction for this purpose: minmerge. It assumes two sorted four element input vectors, $v 0$ and $v 1$, and returns the four smallest elements in sorted order. Two special registers, $n 0$ and $n 1$ (not shown), keep track of how many elements in the output vector originated from $v 0$ and $v 1$ respectively, with the relationship $n 0+n 1=4$. A similar instruction for returning the 4 maximum 


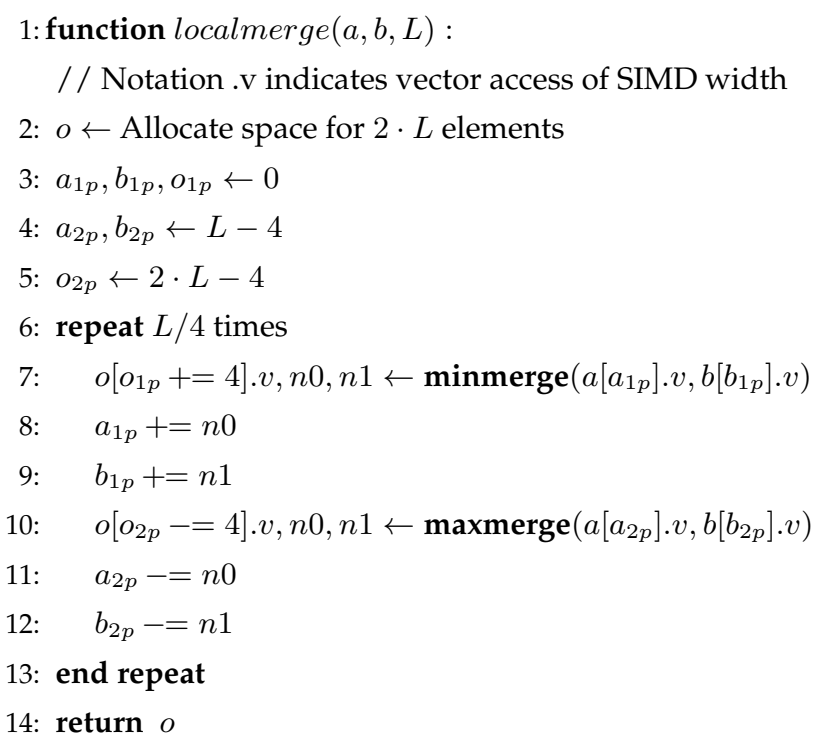

Algorithm 1: Local merging with special merging instructions.

elements (maxmerge) is also useful.

Algorithm 1 shows the algorithm for merging sublists using merge sort with the proposed instructions. In order to avoid going out of bounds on any on the lists, merging is performed both forwards from the start of both lists, and backwards from the ends of both lists. This is also useful to hide pipeline latency. In order to fully hide pipeline latency and to use SIMD instructions for list pointer updates, one can perform multiple merge processes at a time in an interleaved fashion. The proposed algorithm will generally cause unaligned memory accesses and will therefore require a multi-bank memory subsystem or similar to run efficiently.

Multiple sorting implementations have been evaluated for the MPE, to find the most suitable algorithm. This includes the SIMD implementation proposed for the $x 86$ in [159], which is used as our base reference. In addition to this, the use of pure bitonic sorting, pure merge sorting and hybrid bitonic+merge sort have been investigated. It turns out that after the sorted sublists are longer than 64 elements, merge sort performs the best, and which then is the appropriate time to switch algorithm. 
Table 15.13: Relative performance of different sorting implementations on the MPE. The results are normalized to a basic SIMD implementation. The data set is 8192 32-bit integers or floating-point values. ${ }^{*}=$ worst-case/sorting time is input data dependent.

\begin{tabular}{l|ccc}
\hline \hline Kernel & \#LVM & No multi-bank & Multi-bank \\
\hline Reference SIMD min/max & 1 & 1 & 1 \\
with aligned access only & 2 & 1 & 1 \\
\hline Bitonic sort only & 1 & 0.70 & 2.29 \\
& 2 & 1.38 & 4.50 \\
\hline Merge sort only & 1 & $2.58^{*}$ & 4.10 \\
& 2 & $4.26^{*}$ & 6.15 \\
\hline Bitonic + merge sort & 1 & $2.41^{*}$ & 4.28 \\
& 2 & $4.10^{*}$ & 6.70 \\
\hline \hline
\end{tabular}

The relative performance of all implementations is shown in Table 15.13. An LVM memory is large enough to sort up to 8192 elements, which is the data set size used. The effect of using multi-bank memory and the number of LVM memories is investigated. Sorting 8192 32-bit integers or floating-point values takes 314721 cycles using the reference SIMD implementation, regardless of input data. This implementation avoids complex memory access patterns and operates mostly on registers and is therefore not affected by the number of memories or multibank memory. The bitonic sort implementation performs poorly without multi-bank memory, because of the $2^{x}$-strided accesses required to implement the sorting network. However, when using multi-bank memory the performance is up to 4.5 times better than the reference SIMD implementation. Merge sort performs even better with up to 6.15 times better performance than the reference. Note however that without the use of multi-bank memory, sorting time is not predictable. Merge sort accesses multiple consecutive elements, but the input data determines which accesses will span two memory vectors or not. If bitonic sort and merge sort are combined, we achieve up to 6.7 times better performance than 
the reference SIMD implementation. This is equivalent to a cycle time of 46968 cycles.

The presented benchmark clearly shows the impact of multi-bank memory and memory parallelism. However, there is very little benchmark data available for other architectures that sort data sets of this size. We shall return to the topic of general sorting in Chapter 16, where sorting of much larger data sets will be discussed.

\subsubsection{Median filtering}

Non-linear filters are important in image processing, because they can remove image noise while preserving edges and avoid blurring the image [40]. Here we shall investigate the implementation of the median filter.

The median filter replaces each pixel with the median pixel value of an $n \times n$ neighborhood around the pixel. Computing the median requires partial sorting of elements and is noticeably slower than linear filtering on most architectures. The filter kernel must be kept relatively small to achieve good performance. Due to its complexity, it is sometimes implemented in dedicated hardware on custom platforms [160].

The naive way of implementing a median filter is to simply sort each $n \times n$ pixel neighborhood and select the median. It is possible to improve performance considerably only partially sort the data and exploit the overlap between pixel neighborhoods of adjacent pixels. One way of obtaining the $3 \times 3$-median, proposed by [161], is shown in Figure 15.8. It works by first sorting the columns of a pixel block, then sorting the rows and finally sorting the diagonal. The median is then available as the middle element of the sorted diagonal. This algorithm lets us reuse computations, since e.g. the sorted columns is also required for the neighboring blocks. Also, note that we don't need to completely sort the rows, since we are only interested in the maximum, median and minimum value of the upper, middle and lower rows respectively.

Column sorting is easily performed on a SIMD architecture using SIMD min/max instructions, as shown in Algorithm 2. Assuming a SIMD width of 8 and disregarding potential overheads, we have sorted 


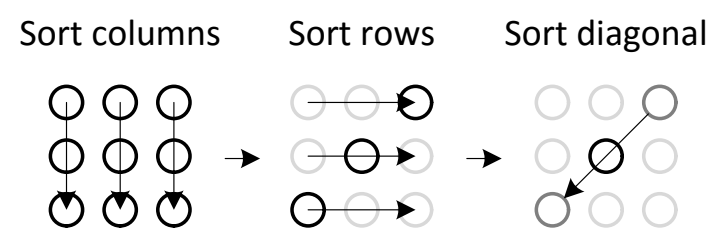

Figure 15.8: Median filtering procedure for a $3 \times 3$ window.
1: $t 1 \min \leftarrow \min ($ row 0, row 1$)$
2: $t 1 \max \leftarrow \max ($ row 0, row 1$)$
3: $t 2 \min \leftarrow \min (t 1 \max$, row 2$)$
4: out $\leftarrow \leftarrow \min (t 1 \min , t 2 \min ) / / \min$ values
5: out $1 \leftarrow \max (t 1 \mathrm{~min}, t 2 \min ) / /$ median values
6: out $2 \leftarrow \max (t 1 \max$, row 2$) / /$ max values

Algorithm 2: SIMD algorithm for column sorting $(3 \times 3$ median kernel).

8 columns after 6 cycles. This is equivalent to $4 / 3$ columns per cycle. If we instead use the already proposed sorting instructions that sort 2 vectors of 4 elements every clock cycle, we reach an equivalent of 2 columns per cycle. In this case we need to utilize the vector masking feature described in Section 11.6.3, to discard 1 of the 4 inputs, since we only sort 3 elements. Still, even if we don't utilize the datapath fully, this represents a speedup of $50 \%$ compared to the SIMD implementation. To complete the kernel implementation, we require the use of permutation vectors to support the required access patterns. Without this feature, the MPE cannot perform better than a standard SIMD implementation.

The median filtering rate for some different architectures is shown in Table 15.14. The main competitors are a SIMD-based architecture, the PowerPC with AltiVec extensions, and the VLIW-based c66x. Even though the data set sizes are odd-numbered and the MPE datapath is not fully utilized, the MPE implementation manages to stay ahead of both these implementations, as well as the low-performance Blackfin. Given the low power consumption of the MPE, we can expect exceptional energy efficiency as well. 
Table 15.14: Comparison of median filtering implementations for 16-bit data.

\begin{tabular}{l|c|c}
\hline \hline Architecture & Filter size & Performance [cycs/pixel] \\
\hline Blackfin [162] & $3 \times 3$ & $\sim 100$ \\
\hline PowerPC [161] & $3 \times 3$ & 2.3 \\
& $5 \times 5$ & 13.2 \\
\hline TI c66x [145] & $3 \times 3$ & 2 (signed) / 3.5 (unsigned) \\
\hline MPE & $3 \times 3$ & 1.583 \\
& $5 \times 5$ & 8.5 \\
& $7 \times 7$ & 20.5 \\
\hline \hline
\end{tabular}

\subsection{General evaluation}

The case studies described in this thesis only cover a small set of the implemented MPE kernels. Since covering the implementation of the complete kernel set is not feasible, this section will try to summarize average results over the kernel set, to draw general conclusions about the MPE, the HOF concept and the proposed local memory subsystem.

In [163] the MPE is compared with the c66x, to draw general conclusions. This was done by implementing over 100 different DSP kernels. The average improvement over all implemented kernels, as well as the results for some common important individual kernels are given in Table 15.15 for 16-bit fixed-point kernels and Table 15.16 for 32-bit floatingpoint kernels. Over all implemented kernels, the MPE reaches an average of 1.7-2.4 times better performance per clock cycle (depending on input data size) than the c66x. This nearly eliminates the 2.5 times clock frequency of the c66x, when the MPE is clocked at $500 \mathrm{MHz}$. Considering that c66 $x^{\prime}$ power consumption is more than one order of magnitude higher than the MPE's, this translates to roughly 10 times better energy efficiency. At the same time, the code size is reduced by $91 \%$ on average.

There are many fine details that can explain the difference in efficiency between the MPE and the c66x. Some of the most important problems with the c66x is the insufficient local memory bandwidth and the 
Table 15.15: Kernel comparison - MPE vs TI c66x for 16-bit fixed-point data. The ranges specified in the Perf./MHz category represent the fact that the relative performance depends on the number of input elements. The ranges assume that the number of input elements is at least 64 .

\begin{tabular}{l|cccc}
\hline \hline Kernel & $\frac{\text { Perf. }}{\text { MHz }}$ & $\frac{\text { Perf. }}{\text { Area }}$ & $\frac{\text { Perf. }}{\text { Watt }}$ & $\begin{array}{c}\text { Code size } \\
\text { reduction }\end{array}$ \\
\hline Autocorr & $1.0-1.7$ & $7-12$ & $8-13$ & $95 \%$ \\
Block move & $2.0-2.3$ & $14-16$ & $15-18$ & $88 \%$ \\
Dot product & $2.0-2.6$ & $14-18$ & $15-20$ & $92 \%$ \\
FIR & $1.5-3.2$ & $10-22$ & $12-25$ & $98 \%$ \\
FIR complex & $1.5-3.0$ & $10-21$ & $12-23$ & $95 \%$ \\
FFT & $1.7-2.4$ & $12-17$ & $13-18$ & $61 \%$ \\
Matrix mul & $2.0-4.2$ & $14-29$ & $15-32$ & $92 \%$ \\
Matrix mul complex & $1.0-4.0$ & $7-28$ & $8-31$ & $92 \%$ \\
Matrix transpose & $3.6-4.0$ & $25-28$ & $28-31$ & $66 \%$ \\
Vector add & $1.5-1.9$ & $10-13$ & $12-15$ & $92 \%$ \\
Vector min/max & $1.0-2.0$ & $7-14$ & $8-15$ & $94 \%$ \\
Vector sum-sq & $2.0-2.3$ & $14-16$ & $15-18$ & $88 \%$ \\
Vector reciprocal & $4.9-6.4$ & $34-45$ & $38-49$ & $84 \%$ \\
\hline \hline
\end{tabular}

fact that the final instruction schedules are not able to fully utilize all resources of the architecture. VLIW schedules can fail practical implementation for many reasons, e.g. insufficient number of registers or simply because all datapaths do not provide the necessary arithmetic capability required for the kernel. In addition, the VLIW architecture has a higher constant overhead with each kernel invocation, primarily because it can take many cycles before some inner-loop software pipeline operates at peak performance. A high constant overhead can reduce efficiency significantly if the input data sets are small.

The advantage of the MPE is not isolated to comparisons with the c66x. Similar results are obtained when the MPE is compared to the Freescale SC3850. The energy consumed per operation is investigated in Table 15.17. The fact that the MPE is implemented with slightly newer 
Table 15.16: Kernel comparison - MPE vs TI c66x for 32-bit floating-point data. The ranges specified in the Perf./MHz category represents the fact that the relative performance depends on the number of input elements. The range assumes that the number of input elements is at least 64 .

\begin{tabular}{l|cccc}
\hline \hline \multirow{2}{*}{ Kernel } & $\frac{\text { Perf. }}{\text { MHz }}$ & $\frac{\text { Perf. }}{\text { Area }}$ & $\frac{\text { Perf. }}{\text { Watt }}$ & $\begin{array}{c}\text { Code size } \\
\text { reduction }\end{array}$ \\
\hline Autocorr & $1.0-1.5$ & $7-10$ & $8-12$ & $95 \%$ \\
Dot product & $2.0-2.1$ & $14-15$ & $15-16$ & $91 \%$ \\
Dot product complex & $0.5-1.0$ & $3-7$ & $4-8$ & $86 \%$ \\
FIR & $1.0-3.2$ & $7-22$ & $8-25$ & $90 \%$ \\
FIR complex & $0.5-1.5$ & $3-10$ & $4-12$ & $89 \%$ \\
Matrix mul & $1.5-3.5$ & $10-24$ & $12-27$ & $90 \%$ \\
Matrix mul complex & $0.6-1.7$ & $4-12$ & $5-13$ & $92 \%$ \\
Matrix transpose & $2.0-3.1$ & $10-24$ & $12-27$ & $38 \%$ \\
Vector add & $1.5-1.7$ & $10-12$ & $12-13$ & $92 \%$ \\
Vector min/max & $1.5-2.0$ & $10-14$ & $12-15$ & $81 \%$ \\
Vector reciprocal & $1.9-2.0$ & $13-14$ & $12-15$ & $96 \%$ \\
\hline \hline
\end{tabular}

Table 15.17: Comparison of energy per operation.

\begin{tabular}{l|ccc}
\hline \hline & \multicolumn{3}{|c}{ Energy consumption $[\mu J]$} \\
Kernel & TI c66x & Freescale SC3850 & ePUMA MPE \\
\hline 1024x32-tap FIR-filter & 11.7 & 10.2 & 0.8 \\
256-point FFT & 0.70 & 0.48 & 0.04 \\
$4 \times 4$ matrix inversion & - & 0.18 & 0.01 \\
\hline \hline
\end{tabular}

technology is not enough to explain the differences in efficiency. One of the most important reasons is naturally that the MPE design can often eliminate the overhead energy of instruction delivery.

The MPE has a large advantage in code size for common DSP kernels. The code size of some common kernels is investigated in [164]. The comparison includes both a vector architecture, a CISC architecture, two RISC architectures and VLIW/SIMD combinations. In addition we add 
Table 15.18: Code size in relation to other types of architectures.

\begin{tabular}{l|cccc}
\hline \hline & \multicolumn{4}{|c}{ Code size [bytes] } \\
Architecture & Filter & RGB2CMYK & Autocorr. & FFT \\
\hline VIRAM (vector) & 708 & 272 & 328 & 720 \\
x86 (CISC) & 944 & 720 & 544 & 15670 \\
PowerPC (RISC) & 1188 & 484 & 1276 & 5868 \\
MIPS (RISC) & 1997 & 1782 & 1137 & 5468 \\
Trimedia (VLIW) & 3584 & 2560 & - & - \\
TI c66x (VLIW) & - & - & 512 & 1504 \\
MPE (HOF) & 96 & 96 & 24 & 288 \\
\hline \hline
\end{tabular}

the size of c66x kernels from [53]. The results are shown in Table 15.18.

The design of the MPE has been guided by our definition of the platform limit, which is to be able to approach the full utilization of the datapath width regardless of data access pattern. The utilization of the datapath for actual compute operations is depicted in Figure 15.9, which is the ratio of compute cycles to total cycles [165]. The utilization is calculated as a function of the number of input elements, or in case of the matrix multiplication and matrix inversion kernels, the number of input matrices. All operations are complex-valued operations. These results demonstrate that the datapath utilization can often approach $100 \%$ as the input data set grows. The auto-correlation kernel is one exception, which is limited by the fact that we need access to the same input data vector two times per cycle (most often at different locations), which is most easily solved by creating a copy of the input vector in a different memory. The matrix inversion kernels are limited by their general implementation complexity and could possibly be improved.

\subsection{Alternative MPE versions}

This section will briefly discuss some alternative versions of the MPE, to demonstrate some practical issues that arise when scaling the architec- 


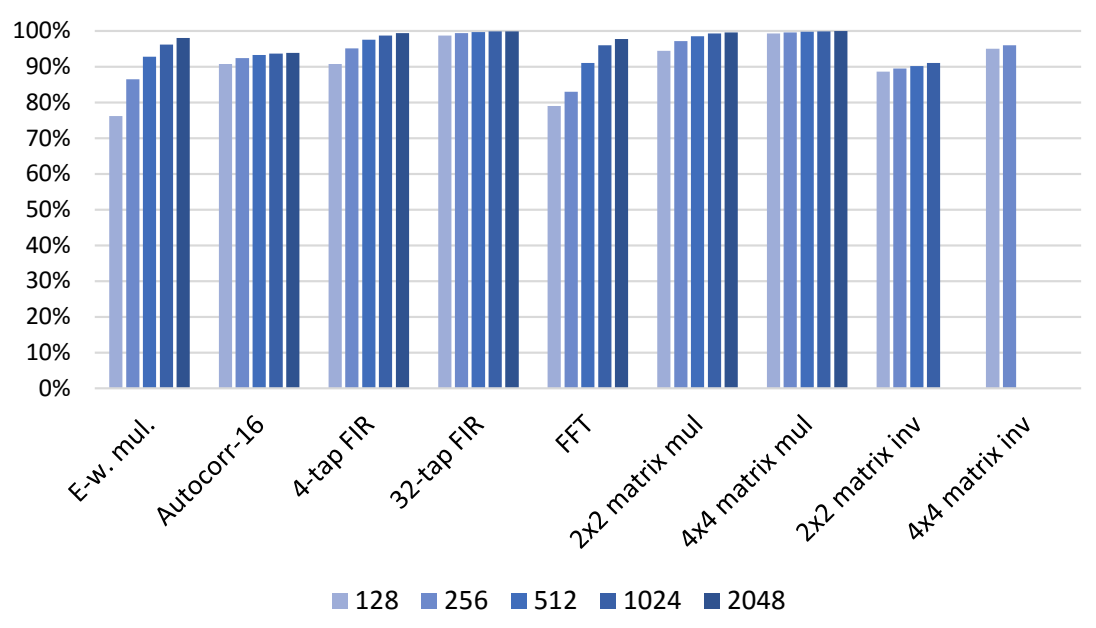

Figure 15.9: MPE efficiency as a function of the number of elements to process (or in case of the matrix operations, the number of matrices to process). Efficiency is defined as the ratio of actual compute cycles to total cycles.

ture, but also to demonstrate the potential benefits.

\subsubsection{Increasing processing width}

The implementation of an MPE with a wider processing width, called MPE-WIDE, has been investigated. This implies that the datapath and memory subsystem are scaled from 128 bit width to some wider width, in this case 256 bits. The intention is to increase the performance without scaling the sizes of memory. This means that the LVM memories instead consist of 16 16-bit banks, but each bank is half of the original size to keep the total amount of memory constant. This modification increases the size of LVM memories by approximately $20 \%$.

The core logic of the MPE becomes approximately $110 \%$ larger with the wider design. The size of the datapath more than doubles. This is because we cannot simply duplicate the original datapath two times, be- 
Table 15.19: Comparison between the 8-way MPE and the 16-way MPE-WIDE.

\begin{tabular}{|c|c|c|}
\hline \multirow[b]{2}{*}{ Parameter } & \multicolumn{2}{|c|}{ Architecture } \\
\hline & MPE & MPE-WIDE \\
\hline Natural vector width & 128 bits & 256 bits \\
\hline Banks per LVM & 8 & 16 \\
\hline Bank size & $8 \mathrm{kB}$ & $4 \mathrm{kB}$ \\
\hline Total size per LVM & $64 \mathrm{kB}$ & $64 \mathrm{kB}$ \\
\hline Total area & $0.43 m m^{2}$ & $0.64 m m^{2}$ \\
\hline Power (estimate) & $70 m W$ & $150 \mathrm{~mW}$ \\
\hline \multicolumn{3}{|c|}{ Benchmarks / Cycle times } \\
\hline Addition int16 & $1 / 8 \mathrm{~N}+7$ & $1 / 16 \mathrm{~N}+8$ \\
\hline Dot product int16 & $1 / 8 \mathrm{~N}+10$ & $1 / 16 \mathrm{~N}+11$ \\
\hline FIR filter cint 16 & $N \cdot\lceil 1 / 4 T\rceil+22$ & $N \cdot\lceil 1 / 8 T\rceil+23$ \\
\hline
\end{tabular}

cause of the reduction instructions. Since we need one additional level of arithmetic units to implement full reduction, one additional level of datapath pipelining may be useful. Other components, such as the register file and AGUs also double in size, while components such as the program flow controller and instruction decoder are less affected.

A few simple performance benchmarks are shown in Table 15.19, together with a comparison of the MPE's and MPE-WIDE's physical characteristics. Quite naturally the performance of most single-instruction kernels doubles, except for one additional cycle of constant overhead due to the prolonged pipeline. Many kernels run without any modifications to the binary kernel code but there are also many exceptions. Kernels that use loop unrolling over the register file may require additional unrolling to perform optimally, since the register file data is consumed in half the number of clock cycles. Kernels that use permutation vectors may need modifications, because the original permutation vector was only 8 elements long or because the permutation table under 16-way processing is not conflict-free. Also, if the inner compute dimension of a HOF instruction is not a multiple of 16 , there may be some additional overhead com- 
Table 15.20: Comparison of the normal MPE with 4 floating-point MAC units and MPE-enhanced with 8 floating-point MAC units.

\begin{tabular}{l|cc}
\hline \hline & \multicolumn{2}{|c}{ Architecture } \\
Parameter & MPE & MPE-enhanced \\
\hline Natural vector width & 128 bits & 128 bits \\
FP-MAC units & 4 & 8 \\
Total area & $0.43 \mathrm{~mm}^{2}$ & $0.48 \mathrm{~mm}^{2}$ \\
\hline \multicolumn{2}{|c}{ Benchmarks / Cycle times } \\
\hline Dot product cfp32 & $1 \mathrm{~N}+11$ & $1 / 2 \mathrm{~N}+12$ \\
FIR filter cfp32 & $N \cdot(T+1)+26$ & $N \cdot(\lceil 1 / 2 T\rceil+2)+27$ \\
\hline \hline
\end{tabular}

pared to 8-way processing. Because 16-way processing was not considered specifically when implementing most kernels in the kernel library, some kernels are not directly compatible, but could be modified with relative ease. Although scaling in this way is not completely free from problems, this can still be considered as a relatively good result, since most other types of architectures would require all kernels to be re-written/recompiled in order to benefit from a wider datapath. This is not the case with the MPE.

\subsubsection{Improved floating-point performance}

The MPE outperforms the c66x in terms of performance per $\mathrm{MHz}$ in most benchmarks. However, there are a few exceptions, namely some complex-valued floating-point benchmarks. As mentioned earlier, this is because the c66x is equipped with 8 FP-MAC units compared to the MPE's 4 . This deficiency can be corrected. By adding 4 separate floatingpoint MAC units, the clock cycle performance of the MPE can surpass the c66x once again. The results are shown in Table 15.20. The cost in terms of hardware area is not too high and the performance is nearly doubled. If complex-valued floating-point processing is critical to the application, this enhancement makes perfect sense. No code modifications were required for these benchmarks. 
Table 15.21: Comparison of the normal MPE and the MPE with advanced floating-point operations support.

\begin{tabular}{l|cc}
\hline \hline & \multicolumn{2}{|c}{ Architecture } \\
Parameter & MPE & MPE-advanced-FP \\
\hline Natural vector width & $128 \mathrm{bits}$ & $256 \mathrm{bits}$ \\
Floating-point ops/cyc & 8 & 40 \\
Complex FP-mults/cyc & 1 & 4 \\
LVM size & $64 \mathrm{kB}$ & $128 \mathrm{kB}$ \\
Total area & $0.43 \mathrm{~mm}^{2}$ & $0.93 \mathrm{~mm}^{2}$ \\
Power (estimate) & $70 \mathrm{~mW}^{2}$ & $200 \mathrm{~mW}$ \\
\hline
\end{tabular}

\section{Benchmarks / Cycle times}

\begin{tabular}{l|cc}
\hline Dot product cfp32 & $1 N+11$ & $N / 4+12$ \\
FIR filter cfp32 & $N \cdot(T+1)+26$ & $N \cdot\lceil T / 4\rceil+27$ \\
\hline \hline
\end{tabular}

\subsubsection{Advanced floating-point MPE}

The MPE versions discussed so far implement only relatively simple SIMD floating-point operations, with the exception of instructions for accelerated sorting. However, it is certainly feasible to implement floatingpoint operations of similar complexity to the fixed-point operations described in this thesis. By replacing the multipliers and arithmetic units in the datapath with 32-bit floating-point multipliers and arithmetic units, we can execute one complex floating-point radix-4 butterfly per cycle. This naturally increases the processing width to 256 bits per cycle.

A floating-point optimized version of the MPE has been investigated. Most of the fixed-point support is removed in favor of floating-point hardware. The structure of the datapath is mostly the same as the fixedpoint version, but some post-processing options are removed. The width of a memory bank is increased from 16 bits to 32 bits, since 16 bit granularity is not required for 32 bit floating-point values. The size of LVM memories are doubled to allow the implementation of the previously implemented 16-bit fixed-point kernels in floating-point without running out of memory. 
Table 15.22: Cycle times for 32-bit complex-valued floating-point FFT.

\begin{tabular}{l|cc}
\hline \hline & \multicolumn{2}{|c}{ Cycle time } \\
Architecture & TI c66x [53] & MPE-advanced-FP \\
\hline 128-point FFT & 590 & 190 \\
256-point FFT & 1144 & 318 \\
\hline \hline
\end{tabular}

Some benchmarks and approximate physical characteristics of the hardware are shown in Table 15.21. Since we haven't had access to appropriate memory macros for the LVMs, we've had to estimate the cost of memory from data sheet data. The design has not been evaluated in layout and the core logic cost is estimated from synthesis. The performance achieved by this version for 32-bit floating-point is naturally quite similar to the performance achieved by the regular MPE for 16 bit fixed-point. The differences are mainly a result of a longer pipeline. For kernels that primarily use SIMD operations, the performance is similar to MPE-WIDE, the MPE with 256 bit vector processing. However, for kernels such as FFT, the performance is significantly improved.

Table 15.22 lists cycle times for 32-bit complex-valued floating-point FFT. The results are as expected given the platform's theoretical peak performance values. The MPE-advanced-FP outperforms the c66x by more than 3 times in terms of performance per clock cycle, which more than enough eliminates the 2.5 times high clock frequency of the c66x. The code of the c66x is roughly 8 times larger than the code of the MPE.

\subsection{Summary}

This chapter evaluated the ePUMA Matrix Processing Element, the HOF concept and the proposed local memory subsystem. The MPE often manages to reach better clock cycle times than competing processor designs and thereby reduces or eliminates their possible advantage in clock frequency. The lower power consumption and hardware area results in very high energy- and area efficiency. The code size reduction com- 
pared to other designs is exceptional, as exemplified by the $91 \%$ average code size reduction over the TI c66x VLIW-DSP. The main reasons for the MPE's success are the efficient encoding of typical computation patterns through HOF instructions, a complex DSP datapath instruction set, a pipeline structure that allows streaming of compute data directly from memory, LUT-based memory access and direct memory permutation on multi-bank memory. This set of features allows us to most often reach our goal: to approach $100 \%$ of the processor's compute capability. 


\section{Chapter 16}

\section{System evaluation}

This chapter will evaluate the complete ePUMA system. Although this is not the main work of the author, it is still important to demonstrate that high efficiency can be maintained at the system level. The main example that will be demonstrated is the implementation of large-scale sorting over the complete platform, using a configuration with 8 MPE processors. Finally, we review the complete system cost of various ePUMA configurations.

\subsection{Sorting on ePUMA}

Sorting is a fundamental operation in computing with many possible uses. In-core sorting can be relatively compute intensive, but when the data set does not fit the local memory or cache of a processor, sorting may instead become limited by communication and memory bandwidth. Because of this, sorting is a benchmark that measures both compute performance, data access performance and other overheads. For best possible performance, the sorting algorithm must be carefully adapted to the target architecture. This section will therefore both discuss design considerations for sorting on distributed memory architectures in general, as well as measure the actual performance achieved by ePUMA.

The possibly most complex design choice when implementing sorting is what sorting algorithm to use. Chapter 15 has already demon- 
strated how core-level sorting may be accelerated by using applicationspecific sorting instructions and multi-bank memory. In this implementation, bitonic sort and merge sort were combined for best results. At the system-level, bitonic sorting is not a good choice, because it leads to high communication overhead $[62,166]$. A popular alternative choice is radix sort [167]. This works well for general-purpose CPUs and GPUs, but the scattered writes over main memory makes it less suitable for ePUMA. The system-level sorting algorithm for ePUMA is therefore based on merge sort.

An important aspect to consider for distributed memory architectures is that data transfers must be handled explicitly. In addition, to hide memory transfer latency we would like to know what data to load long before the data is actually needed. For best utilization of main memory, it is preferable to request data in larger batches. At the same time, we should ensure that we are not limited by memory bandwidth.

The system-level sorting algorithm for ePUMA is shown in Algorithm 3. The input parameters are:

- Array A: The array of elements to sort.

- Scalar N: The total number of elements to sort. We assume that $N \in\left\{2^{x}: x \in \mathbb{N}\right\}$.

- Scalar M: In-core phase block size.

- Scalar S: Out-of-core phase block size.

The sorting procedure is divided in two phases: the in-core phase and the out-of-core phase. This approach is virtually the same as many other merge sort implementations. The in-core phase divides the total set of $N$ elements into smaller chunks of $M$ elements, which fit in processor local memories. All $N / M$ chunks are processed individually using the function MPESort, which is the in-core sorting kernel from Chapter 15. This phase is massively data parallel and can be implemented using a blockmap algorithmic skeleton. Data transfers can be overlapped with sorting, since new input data can be loaded to a spare LVM memory while a previously loaded data set is sorted. The out-of-core phase merges the $N / M$ 


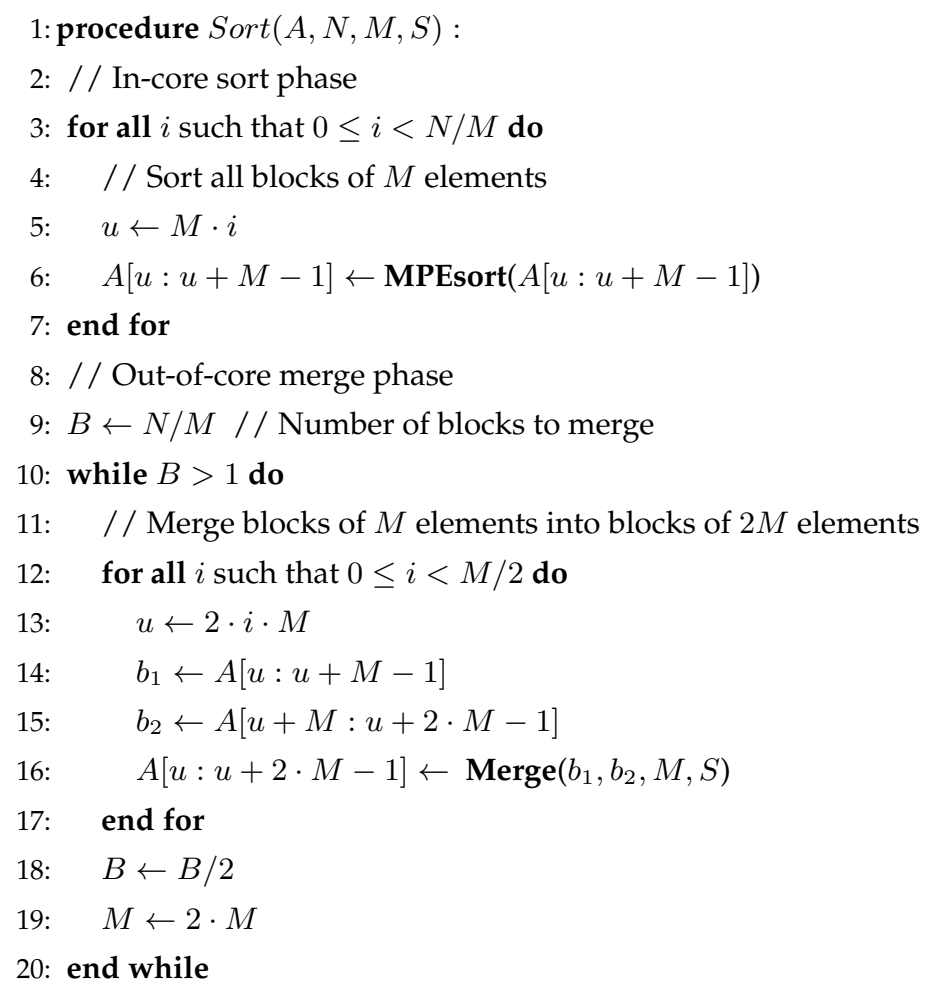

Algorithm 3: The proposed system-level sorting algorithm. The input parameters are: $A$ - the list to sort, $N$ - the size of list $A, M$ - in-core phase block size and $S$ - out-of-core phase block size.

sorted chunks into the final sorted list. However, since complete merge sets can no longer fit into a single LVM memory, special care must be taken.

Algorithm 3 describes the high-level out-of-core procedure using 2way merging, but the important details are hidden within the Merge function. The implementation of Merge is shown in Algorithm 4. The algorithm merges two lists in main memory by repeatedly merging $S$ elements from one of the two lists with a temporary list of $S$ maximum values $l_{\text {max }}$. Two pointers, $b_{1 p}$ and $b_{2 p}$, keep track of the merging process. $S$ new elements are selected for merging based on the value of the 


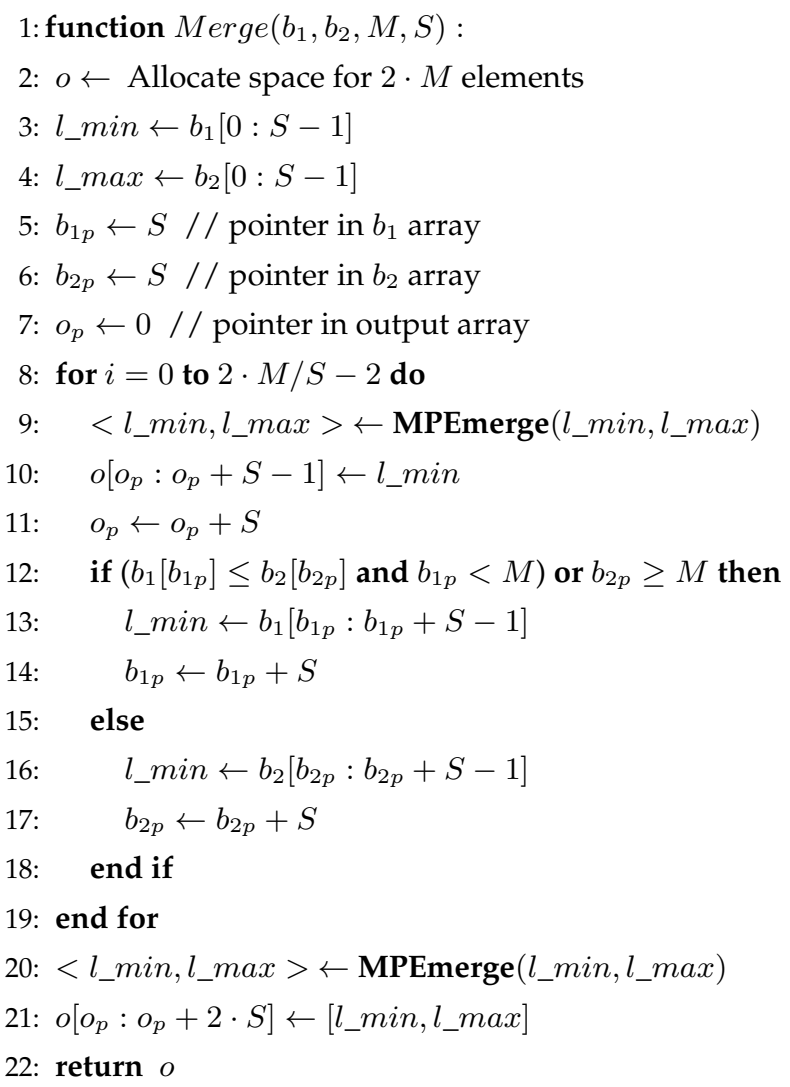

Algorithm 4: The system-level merge algorithm.

elements the pointers are currently pointing to. The kernel MPEMerge from Chapter 15 is used to merge a new input list with the temporary list. Note how line 9 represents local merging, lines 10-11 stores the results of a merge phase back to main memory and lines 12-18 represent loading of new input data from main memory. By inserting temporary buffers, these processes can be overlapped.

Algorithm 4 solves the problem of finding large fixed-size data blocks to transfer between LVM memories and main memory. The selection of the next set of elements to merge is based on comparisons (line 12), which in some sense represents partial sorting. However, if $S$ is selected to be 
at least hundreds of elements, this partial sorting is small compared to the total sorting. It is worth noting that similar blockwise sorting processes have been suggested before [168, 169]. In these cases $S$ has been selected to be very small, typically 4 or 8 , which is used for the purpose of avoiding unaligned memory accesses in SIMD implementations.

\subsubsection{Multi-way merging}

The most straight-forward approach to out-of-core merging is to merge two lists at a time. However, this means that memory bandwidth requirements are high. By replacing 2-way merging with multi-way merging, bandwidth requirements are reduced [159]. In addition, since main memory accesses are expensive in terms of energy, energy efficiency may be improved. Architectures with efficient inter-processor communication capabilities can do multi-way merging over multiple cores as proposed for the Cell BE architecture [166]. This approach is applied in the ePUMA sorting implementation as well, but only for the last few stages of sorting, when there are only a few large lists left to merge. Otherwise, independent multi-way merging on multiple cores turns out to be faster because all cores are easily utilized and no inter-processor communication overheads are present.

Algorithm 4 is easily extended into multi-way merging as follows: Consider the 4-way merger in Figure 16.1. At the bottom merge node, one of the two input lists should be merged with the current local maximums, based on a comparison between the first element of both input lists. Once an input list has been selected, a predecessor merge node loses its previously generated output list and may produce a new one. This procedure is repeated until the top of the tree is reached, where a new input list must be loaded from main memory. If appropriate buffering is inserted, all merging processes can run simultaneously. This is used to make sure that the processor pipeline is filled with work. Additionally, merging can be performed both from the beginning of both lists as well as the ends to find even more work. The same procedure is used in combination with on-chip pipelining for the final merging layers. 


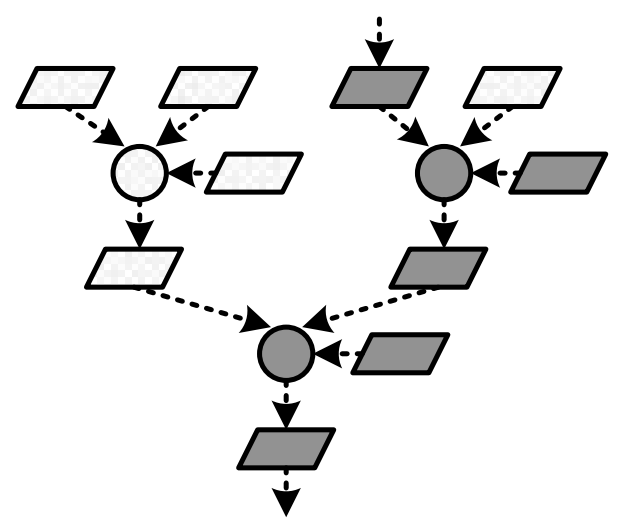

Figure 16.1: A 4-way merging setup.

Choosing the correct multi-way merging factor is a trade-off between processing efficiency and main memory bandwidth usage. A higher value means less overall main memory data transfers. However, this means that the value of $S$ has to be small so that all intermediate data can fit in an LVM. The consequence is that each kernel invocation has less data to process, which means that we must call the kernel more times and each call has a certain overhead. For ePUMA, it turns out that 16way merging performs the best given the available memory bandwidth and a total of 8 MPEs.

\subsubsection{Evaluation}

Due to the lack of an ePUMA VLSI chip, the system evaluation has been done on an ePUMA FPGA prototype. The prototype uses 4 compute clusters, each equipped with 2 MPEs. This happened to be the maximum number of MPEs that could fit on the FPGA, but this also turns out the maximum number of cores that can be efficiently exploited using 16-way multi-way merging and the available memory bandwidth. The sorting times achieved on the FPGA board have then been scaled to the VLSI design frequency of $500 \mathrm{MHz}$ and the power consumption of the chip was estimated from the VLSI layout. In practice there will be dif- 
Table 16.1: Hardware parameters for the investigated systems.

\begin{tabular}{l|ccccc}
\hline \hline System & $\begin{array}{c}\text { Ops/s } \\
\text { [GFLOPS] }\end{array}$ & $\begin{array}{c}\text { MemBW } \\
{[\text { GB/s] }}\end{array}$ & $\begin{array}{c}\text { Tech. } \\
{[\mathbf{n m}]}\end{array}$ & $\begin{array}{c}\text { Area } \\
{\left[\mathbf{m m}^{2}\right]}\end{array}$ & $\begin{array}{c}\text { Power } \\
{[\mathbf{W}]}\end{array}$ \\
\hline ePUMA & 32 & 8 & 28 & 5.6 & 0.6 \\
Core i7 & 102.4 & 30 & 45 & 263 & 104 \\
Xeon Phi & 2020 & 320 & 22 & 350 & 180 \\
GeForce Titan & 4500 & 288 & 28 & 561 & 200 \\
Cell BE & 204.8 & 25.6 & 45 & 115 & 40 \\
\hline \hline
\end{tabular}

ferences in main memory characteristics between the FPGA implementation and a VLSI chip. However, given that the sorting implementation is latency-tolerant by design and that the rest of the systems are identical, the results should not differ significantly.

Table 16.1 lists the systems we compare our implementation with. Since neither the sorting implementations nor the actual platforms have been available to us, the power consumption values given are very rough estimates of actual system power consumption, based on manufacturerprovided maximum power or TDP values [170, 171, 62]. It should also be noted that the power consumption for the GPU includes all components on the GPU's PCB, while the other systems only measure chip power consumption. It is clear from the stated specifications that all competing systems are theoretically much more powerful than ePUMA. On the other hand, ePUMA is a much smaller design and has a much lower power consumption.

Figure 16.2 shows the sorting rate in millions of keys sorted per second for various sorting implementations. The data type and sorting algorithm used are indicated. All implementations sort 32-bit integers or 32-bit floating-point values. The performance of ePUMA is virtually the same regardless of data type, because of the sorting network instructions. The GeForce Titan is clearly the fastest, followed by the Xeon Phi. ePUMA demonstrates performance similar to the Core i7. Given the much higher theoretical performance values of the competing platforms, 


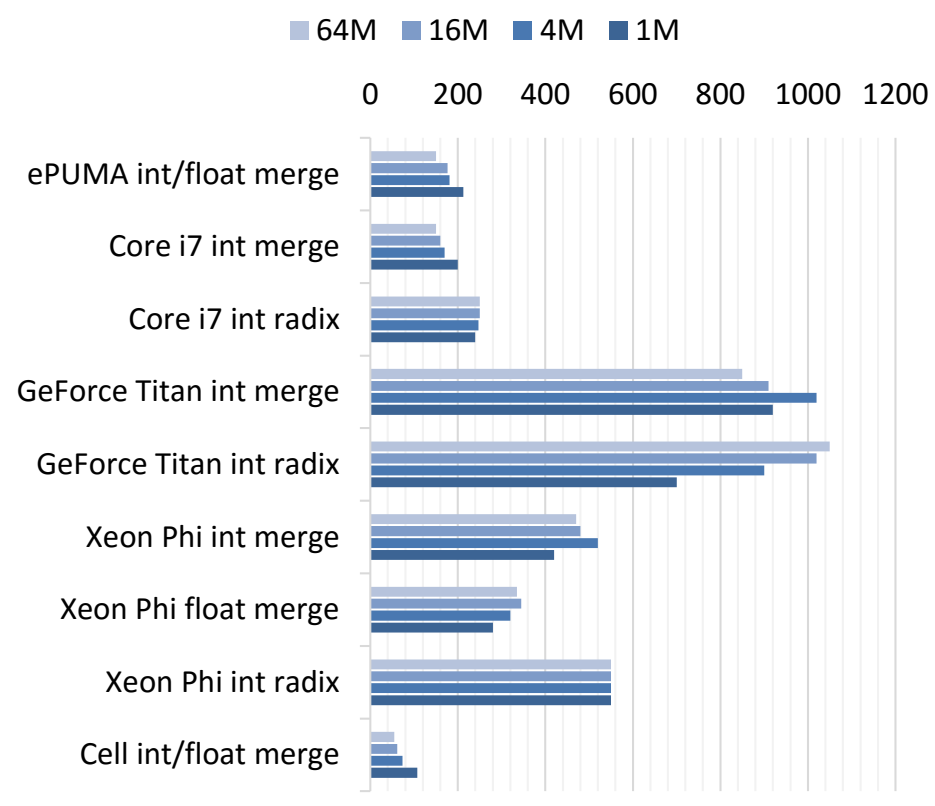

Figure 16.2: Sorting rate in millions of keys sorted per second, as a function of the total number of elements to sort.

we can clearly see that the ePUMA implementation uses its hardware more effectively.

Table 16.2 lists the sorting rate over hardware area and power. The results are shown both at each system's native technology, as well as scaled to $28 \mathrm{~nm}$, which is the technology used for ePUMA. Area is scaled quadratically, while power is scaled quadratically for older technologies and linearly for newer technologies, simply to avoid overestimating the results of ePUMA. Since some of the competing implementations use relatively old platforms, the performance of some newer more energy efficient systems have been estimated, including the ultra-low-power Core $\mathrm{M}$, with a maximum rated power consumption of $4.5 \mathrm{~W}$. Although these results are largely based on estimated power consumption of all systems, ePUMA is clearly superior in Mkeys/s sorted per Watt by roughly two 
Table 16.2: Average sorting rate over area and power for the investigated systems. Types int and $f p$ represent integer and floating-point sorting respectively. Algorithm $b+m$ represents algorithms based on bitonic sort and merge sort, while radix represents radix sort.

\begin{tabular}{|c|c|c|c|c|}
\hline \multirow[b]{2}{*}{ System } & \multirow[b]{2}{*}{ Type } & \multirow[b]{2}{*}{ Alg. } & \multicolumn{2}{|c|}{ Native tech./Scaled to $28 \mathrm{~nm}$} \\
\hline & & & {$\left[\mathrm{Mkeys} / \mathrm{s} / \mathrm{mm}^{2}\right]$} & [Mkeys/s/Watt] \\
\hline $\begin{array}{l}\text { ePUMA } \\
\text { [6] }\end{array}$ & int/fp & $\mathrm{b}+\mathrm{m}$ & 33 & 305 \\
\hline Core i7 & int & $b+m$ & $0.64(45 \mathrm{~nm}) / 1.66$ & $1.64 / 4.20$ \\
\hline [159] & int & radix & $0.94(45 \mathrm{~nm}) / 2.42$ & $2.37 / 6.11$ \\
\hline GeForce Titan & int & $\mathrm{b}+\mathrm{m}$ & 1.65 & 4.64 \\
\hline [172] & int & radix & 1.65 & 4.61 \\
\hline Xeon Phi & int & $b+m$ & $1.37(22 \mathrm{~nm}) / 0.84$ & $2.65 / 1.64$ \\
\hline [172] & $\mathrm{fp}$ & $b+m$ & $0.92(22 \mathrm{~nm}) / 0.57$ & $1.79 / 1.41$ \\
\hline & int & radix & $1.57(22 \mathrm{~nm}) / 0.97$ & $3.06 / 2.40$ \\
\hline $\begin{array}{l}\text { Cell BE } \\
{[62,166]}\end{array}$ & int/fp & $\mathrm{b}+\mathrm{m}$ & $0.65(45 \mathrm{~nm}) / 1.67$ & $1.86 / 4.81$ \\
\hline $\begin{array}{l}\text { Core M-5Y70 } \\
\text { (estimated) }\end{array}$ & int & $\mathrm{b}+\mathrm{m}$ & $3.00(14 \mathrm{~nm}) / 0.75$ & $34.17 / 17.08$ \\
\hline $\begin{array}{l}\text { GeForce 980M } \\
\text { (estimated) }\end{array}$ & int & $\mathrm{b}+\mathrm{m}$ & 1.64 & 6.54 \\
\hline
\end{tabular}

orders of magnitude. These results are by far the best sorting performance over area and power from a programmable processor known by the author.

\subsection{Other algorithms and applications}

In addition to the implementation of sorting, many other system-level algorithms and applications have been implemented. The author has been directly or indirectly involved in other implementations, such as motionJPEG encoding [143], MPEG decoding [173], H.264 encoding [98], Long 
Term Evolution (LTE) processing [7], Euclidian distance transform calculation [174] and ePUMA for low-end graphics acceleration [175, 176, 177]. In addition, the author has implemented system-level code for sparse matrix operations, image filtering, 2D-FFT and feature detection. This work remains to be published.

\subsection{System hardware evaluation}

We conclude this chapter by estimating the characteristics of some physical ePUMA implementations. By being a configurable platform, the specifications can vary greatly. Table 16.3 lists three possible configurations of very different performance. All configurations use a complete MPE instruction set for general-purpose processing. We simply scale the clock frequency, the number of compute clusters, the number of MPEs per compute cluster and the size of memories. The configuration High-perf. uses a clock frequency of $800 \mathrm{MHz}$ which hasn't been verified in layout, but synthesis suggests that it possible to run the system at $800 \mathrm{MHz}$ and beyond.

It is worth noting that the total main memory bandwidth is only scaled with the system clock frequency, since an off-chip main memory bandwidth of 128-bits per clock cycle is assumed. This means that as the number cores is scaled, there is less memory bandwidth per core. In order to fully utilize a configuration such as High-perf., the application must have a very high compute-to-bandwidth ratio, otherwise the main memory bandwidth must be scaled further. It should however be noted that a DSP system may have many other interface ports in addition to the main memory interface, which means that the actual total I/O bandwidth of the chip could be significantly higher.

\subsection{Summary}

This chapter evaluated the efficiency of a complete ePUMA through an implementation of large scale sorting. The implementation suggests that 
Table 16.3: Hardware specifications for some possible homogeneous ePUMA configurations, including approximate hardware cost and power consumption.

\begin{tabular}{|c|c|c|c|c|}
\hline Parameter & $\begin{array}{l}0 \\
0 \\
0 \\
0 \\
0 \\
0 \\
0 \\
0\end{array}$ & $\begin{array}{l}\text { 로 } \\
\text { ఏू̉ } \\
\text { Z }\end{array}$ & 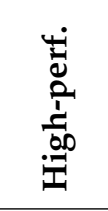 & Unit \\
\hline Clock freq. & 200 & 500 & 800 & $M H z$ \\
\hline Master I-cache & 16 & 64 & 1024 & $k B$ \\
\hline Master D-cache & 16 & 64 & 1024 & $k B$ \\
\hline LVM size & 8 & 64 & 64 & $k B$ \\
\hline eCCC-LS size & 8 & 32 & 64 & $k B$ \\
\hline MPE-LS size & 4 & 16 & 32 & $k B$ \\
\hline \# CCs & 2 & 4 & 8 & \\
\hline \# LVMs/CC & 3 & 6 & 24 & \\
\hline \# MPEs/CC & 1 & 2 & 8 & \\
\hline Int.ops/cyc & 16 & 160 & 2048 & GIOPS \\
\hline Float.ops/cyc & 3.2 & 32 & 409.6 & GFLOPS \\
\hline Total LVM bandwidth & 18.75 & 187.5 & 2400 & $G B / s$ \\
\hline Total MM bandwidth & 3.125 & 7.8 & 12.5 & $G B / s$ \\
\hline Total LVM memory & 48 & 1536 & 12288 & $k B$ \\
\hline Total memory & 104 & 2048 & 16896 & $k B$ \\
\hline Core area & 0.8 & 1.9 & 11 & $m m^{2}$ \\
\hline Memory area & 1 & 3.7 & 34 & $m m^{2}$ \\
\hline Total area & 1.8 & 5.6 & 45 & $m m^{2}$ \\
\hline Total power (estimate) & 130 & 825 & 7700 & $m W$ \\
\hline
\end{tabular}


the high efficiency of kernels can be maintained at the system level as well, if the system-level application is designed appropriately. The energy efficiency of ePUMA compared to contemporary high-performance general-purpose CPUs and GPUs suggests an advantage of up to two orders of magnitude.

In addition to the implementation of sorting, other important work on ePUMA was highlighted. Finally, the characteristics of a few different ePUMA configurations were given, showing the high potential scalability of the platform. 


\section{Part VI}

\section{Conclusions and future work}





\section{Chapter 17}

\section{Conclusions}

The purpose of the ePUMA project was to investigate how to design energy-efficient configurable DSP platforms for predictable DSP computing. The most important design criteria were to approach the computing limit of the datapath by avoiding data access conflicts and at the same time maintain high energy efficiency and performance predictability. These criteria lead to the introduction of HOF instructions and distributed multi-bank memory with permutation-based memory access. Compared to contemporary DSP designs, our approach is to introduce increasingly powerful datapath operations, rather than exploiting more instruction-level parallelism. By doing so we can increase computing performance with low energy overhead, instead of increasing the energy consumption of instruction fetching, decoding and pipeline control.

This research, which primarily focuses on core-level issues, demonstrates the benefits of the presented approaches through compute kernel implementation. This implies that the research can truly never be complete: there are certainly more interesting kernels to implement, which may still affect core design issues. However, given the relatively large kernel library that has been implemented, the generality of the presented approaches seems to hold well within the domain of DSP processing. 


\subsection{Computing- and energy efficiency}

Increasing the performance capability by adding more powerful instructions seems to be a good approach for any computing area and is by no means unique for this research. By encoding more work per delivered instruction, the hardware designer can optimize the execution of such computing patterns in ways software implementations may not be able to. The MPE kernel library indicates that the compute kernel library of a HOF-based processor can more often reach the platform limit than e.g. a VLIW-based processor. Since this research implements the same kernels that VLIW processor manufacturers consider important, this is a very encouraging result. A VLIW architecture may be more flexible, but this research indicates that such high flexibility is unnecessary for the implementation of compute kernels. Improvements in computing efficiency directly translates into potential energy reduction, such as a reduced clock frequency. The potential reductions in code size is an added bonus, which makes it possible to keep more kernels in local memory and reduce traffic to the very critical main memory.

\subsection{Predictability}

The ePUMA design has put much focus on performance predictability. The use of program-controlled distributed memory means that data can be prepared before use to eliminate global data access overhead. When data is available locally close to processor cores, kernel execution time is predictable or even fixed, and most often data oblivious. For some applications, predictability may be of extreme importance and many current cache-based designs are hard to use under such a constraint, or require a programming effort similar to low-level ePUMA programming anyway. Compared to many other designs, the ePUMA design can generally provide much stronger guarantees, at least as long as off-chip main memory is not the bottleneck. This may simplify the use in hard real-time systems. 


\subsection{Final remarks}

It has certainly been a rewarding experience to work on a full DSP system, both from a hardware perspective and a software perspective. Since the goal has been to produce a fully working system and tool-chain, no problems could be pushed aside. A quick solution to a hardware problem can easily result in a very complex software implementation or vice versa. Careful co-design of all components is required, as well as very active collaboration within the design team. Although there is still much work left to do, the main goals of this particular research have been reached and many interesting opportunities for future work have been identified. 


\section{Chapter 18}

\section{Future work}

This chapter will summarize the most interesting directions for future work in the opinion of the author.

\subsection{Hardware aspects}

There are plenty of opportunities for further investigation of hardware platforms. One such opportunity is to apply the HOF concept in a shared memory system. This work has already been initiated by implementing a shared memory version of ePUMA in simulation. By using shared memory, programming may be simplified and more programming models may be applicable. At the same time, distributed LVMs may be used as temporary computing buffers closely attached to the MPE processors. In this way, one may combine the benefits of shared memory and distributed memory. The cost is a larger hardware area and reduced predictability, but for many applications this trade-off is a reasonable one to make.

The ePUMA platform covers predictable DSP computing including coarse-grained control by control processors. However, with new emerging DSP applications there may be needs for fast execution of fine-grained control structures at the kernel-level. Merging such structures into complex multi-cycle instructions, in a similar way as HOF instructions encode well-behaved compute structures, is most likely much more in- 
volved. Reducing energy consumption overhead from e.g. instruction fetching may prove very challenging in this case.

Another direction for increasing the flexibility of a HOF-based architecture, is to combine the $\mathrm{HOF}$ concept with e.g. superscalar processing, or simply let HOF instructions act as an extension to some other instruction paradigm. One may for instance continue to fetch and issue independent instructions, while a many-cycle HOF instruction is already executing. In this way, it may be possible to hide the overheads of more complex control structures and even let a HOF-based processor act as a control processor.

The current ePUMA hardware has very limited power management, besides the fine-grained clock gating applied by the hardware synthesis tool. A real system will require additional features. However, this work has primarily focused on online energy optimizations at the architectural level. Fine-grained gate-level optimizations have not been investigated. Reducing offline energy consumption and dynamic voltage and frequency scaling is left as future research.

To fully prove the energy benefits of the demonstrated hardware concepts, a chip tape-out would be required. The energy estimates from simulation has had to suffice and the FPGA prototype has demonstrated hardware correctness. Although absolute energy values may not be perfectly accurate from simulation, at least relative ones should provide good enough predictions of real hardware.

\subsection{System tool-chain}

It is the personal belief of the author that the most challenging problems yet to solve are not directly related to hardware, but rather to the parallel programming methodology and tools. The eOctave DSL and compiler for the MPE is not complete and needs further work. HOF instructions introduce new compiler optimization problems, which currently are solved with simple heuristics. Currently, many problems are left for the programmer to solve, such as arranging kernel computations for long 
vector execution. The current kernel compiler is unable to utilize all potential addressing capabilities of the MPE, which sometimes forces the use of assembly programming for best performance. These problems are still under investigation.

A system-level programming model with a higher level of abstraction would be very useful to the ePUMA system. The investigations of TensorFlow and CAL is ongoing, but the end results remain unclear. Hopefully the results of these efforts can be presented in the near future.

\subsection{Application development}

Although there are still many interesting kernels left to implement, application development is now the main priority. Several applications have been implemented on various versions of the ePUMA hardware, which must be updated to the latest design for full evaluation. Additionally, we hope to be able to map more applications in the future, especially when the tool-chain becomes more mature.

\subsection{Release of ePUMA}

The hardware platform, the simulators, the $\mathrm{C}$ compilers for the master processor and cluster controllers and the MPE kernel library have now reached such a stable state that they may be used by others. There are plans to release ePUMA to the general public, after some final documentation is ready. In this way, the ePUMA platform can continue to exist and be improved, even after the conclusion of the presented work. 


\section{References}

[1] G. E. Moore, "Cramming more components onto integrated circuits," Electronics, vol. 38, no. 8, pp. 114-117, 1965.

[2] R. H. Dennard, F. H. Gaensslen, V. L. Rideout, E. Bassous, and A. R. LeBlanc, "Design of ion-implanted MOSFET's with very small physical dimensions," IEEE Journal of Solid-State Circuits, vol. 9, no. 5, pp. 256-268, Oct. 1974.

[3] International Technology Roadmap for Semiconductors, "ITRS Executive Summary," Tech. Rep., 2013. http:/ /www.itrs2.net/

[4] D. Liu, Embedded DSP Processor Design: Application Specific Instruction Set Processors. San Francisco, CA, USA: Elsevier Science, 2008.

[5] D. Liu, A. Karlsson, J. Sohl, J. Wang, M. Petersson, and W. Zhou, "ePUMA embedded parallel DSP processor with unique memory access," in $8^{\text {th }}$ International Conference on Information, Communications \& Signal Processing, Dec. 2011, pp. 1-5.

[6] A. Karlsson, J. Sohl, and D. Liu, "Energy-efficient sorting with the distributed memory architecture ePUMA," in IEEE Trustcom/BigDataSE/ISPA, Aug. 2015.

[7] J. Wang, "Low overhead memory subsystem design for a multicore parallel DSP processor," Ph.D. dissertation, Linköping University, 2014. 
[8] J. Sohl, "Efficient compilation for application specific instruction set DSP processors with multi-bank memories," Ph.D. dissertation, Linköping University, 2015.

[9] A. Nilsson, "Design of programmable multi-standard baseband processors," Ph.D. dissertation, Linköping University, Sweden, 2007.

[10] E. Tell, "Design of programmable baseband processors," Ph.D. dissertation, Linköping University, Sweden, 2005.

[11] Research Group at Div. of Computer Engineering, ISY, Linköping University, "ePUMA reference manual," Tech. Rep., 2014. http://www.da.isy.liu.se/research/ePUMA/

[12] Research Group at Div. of Computer Engineering, ISY, Linköping University, "ePUMA MPE: Kernel library reference," Tech. Rep., 2015. http://www.da.isy.liu.se/research/ePUMA/

[13] Research Group at Div. of Computer Engineering, ISY, Linköping University, "ePUMA MPE: Instruction set reference," Tech. Rep., 2015. http://www.da.isy.liu.se/research/ePUMA/

[14] J. M. Rabaey, A. P. Chandrakasan, and B. Nikolic, Digital Integrated Circuits: A Design Perspective. Pearson Education, 2003.

[15] I. L. Markov, "Limits on fundamental limits to computation," $\mathrm{Na}$ ture, vol. 512, no. 7513, pp. 147-154, Aug. 2014.

[16] S. W. Keckler, W. J. Dally, B. Khailany, M. Garland, and D. Glasco, "GPUs and the future of parallel computing," IEEE Micro, vol. 31, no. 5, pp. 7-17, Sep. 2011.

[17] S. Borkar and A. A. Chien, "The future of microprocessors," Communications of the ACM, vol. 54, no. 5, pp. 67-77, May 2011.

[18] H. Esmaeilzadeh, E. Blem, R. St. Amant, K. Sankaralingam, and D. Burger, "Dark silicon and the end of multicore scaling," in Pro- 
ceedings of the $38^{\text {th }}$ Annual International Symposium on Computer Architecture, San Jose, CA, USA, 2011, pp. 365-376.

[19] M. B. Taylor, "A landscape of the new dark silicon design regime," IEEE Micro, vol. 33, no. 5, pp. 8-19, 2013.

[20] M. Johnsson, Superscalar Microprocessor Design. Prentice Hall, 1990.

[21] ARM, “NEON programmer's guide, version 1.0,” Tech. Rep., 2015.

[22] Intel, "Intel 64 and IA-32 architectures, instruction set reference, A-Z," Tech. Rep.

[23] D. Talla, L. K. John, and D. Burger, "Bottlenecks in multimedia processing with SIMD style extensions and architectural enhancements," IEEE Transactions on Computers, vol. 52, no. 8, pp. 10151031, 2003.

[24] S. Maleki, Y. Gao, M. J. Garzarán, T. Wong, and D. A. Padua, "An evaluation of vectorizing compilers," in Proceedings of the International Conference on Parallel Architectures and Compilation Techniques, 2011, pp. 372-382.

[25] C. H. K. van Berkel, "Multi-core for mobile phones," in Design, Automation \& Test in Europe Conference \& Exhibition, Nice, France, Apr. 2009, pp. 1260-1265.

[26] R. Kumar, K. I. Farkas, N. P. Jouppi, P. Ranganathan, and D. M. Tullsen, "Single-ISA heterogeneous multi-core architectures: The potential for processor power reduction," in Proceedings of the $36^{\text {th }}$ Annual IEEE/ACM International Symposium on Microarchitecture, 2003, pp. 81-92.

[27] N. Goulding-Hotta, J. Sampson et al., "GreenDroid: An architecture for the dark silicon age," in $17^{\text {th }}$ Asia and South Pacific Design Automation Conference, Jan. 2012, pp. 100-105. 
[28] D. Sorin, M. Hill, and D. Wood, A Primer on Memory Consistency and Cache Coherence. Morgan and Claypool, 2011.

[29] J. D. Balfour, "Efficient embedded computing," Ph.D. dissertation, Stanford University, 2010.

[30] R. Hameed, W. Qadeer et al., "Understanding sources of inefficiency in general-purpose chips," in Proceedings of the $37^{\text {th }}$ Annual International Symposium on Computer Architecture, Saint-Malo, France, 2010, pp. 37-47.

[31] C. Isci, A. Buyuktosunoglu, C.-y. Cher, P. Bose, and M. Martonosi, "An analysis of efficient multi-core global power management policies: Maximizing performance for a given power budget," in $39^{\text {th }}$ Annual IEEE/ACM International Symposium on Microarchitecture, Dec. 2006, pp. 347-358.

[32] H. Kawaguchi and T. Sakurai, "A reduced clock-swing flip-flop (RCSFF) for 63\% power reduction," IEEE Journal of Solid-State Circuits, vol. 33, no. 5, pp. 807-811, May 1998.

[33] M. Kondo, H. Kobyashi et al., "Design and evaluation of finegrained power-gating for embedded microprocessors," in Design, Automation \& Test in Europe Conference \& Exhibition, Mar. 2014, pp. $1-6$.

[34] D. Liu, "Baseband ASIP design for SDR," China Communications, vol. 12, no. 7, pp. 60-72, Jul. 2015.

[35] D. Wu, J. Eilert, D. Liu, A. Nilsson, E. Tell, and E. Alfredsson, "System architecture for 3GPP LTE modem using a programmable baseband processor," in International Symposium on System-on-Chip, Oct. 2009, pp. 132-137.

[36] Y. Sun and J. R. Cavallaro, "Efficient hardware implementation of a highly-parallel 3GPP LTE/LTE-advance turbo decoder," Integration, the VLSI Journal, vol. 44, no. 4, pp. 305-315, Sep. 2011. 
[37] FFmpeg, “FFmpeg," 2010. http://www.ffmpeg.org

[38] H. Chang, J. Cho, and W. Sung, "Compiler-based performance evaluation of an SIMD processor with a multi-bank memory unit," Journal of Signal Processing Systems, vol. 56, no. 2-3, pp. 249-260, Jun. 2009.

[39] G. J. Sullivan, J.-R. Ohm, W.-J. Han, and T. Wiegand, "Overview of the high efficiency video coding (HEVC) standard," IEEE Transactions on Circuits and Systems for Video Technology, vol. 22, no. 12, pp. 1649-1668, Dec. 2012.

[40] R. C. Gonzalez and R. E. Woods, Digital Image Processing, 3rd ed. Upper Saddle River, NJ, USA: Prentice-Hall, Inc., 2006.

[41] OpenCV reference manual 3.0. http://www.opencv.org

[42] BDTI. BDTI benchmarks. http://www.bdti.org

[43] R. Banakar, S. Steinke, B.-S. Lee, M. Balakrishnan, and P. Marwedel, "Scratchpad memory: Design alternative for cache on-chip memory in embedded systems," in Proceedings of the 10th International Symposium on Hardware/Software Codesign, Estes Park, Colorado, 2002, pp. 73-78.

[44] W. Dally, J. Balfour et al., "Efficient embedded computing," Computer, vol. 41, no. 7, pp. 27-32, Jul. 2008.

[45] D. T. Wang, "Modern DRAM memory systems: Performance analysis and scheduling algorithm," Ph.D. dissertation, University of Maryland, College Park, MD, USA, 2005, aAI3178628.

[46] J. Sohl, J. Wang, A. Karlsson, and D. Liu, "Automatic permutation for arbitrary static access patterns," in IEEE $10^{\text {th }}$ International Symposium on Parallel and Distributed Processing with Applications, Jul. 2012, pp. 215-222. 
[47] J. Sohl, J. Wang, A. Karlsson, and D. Liu, “Conflict-free data access for multi-bank memory architectures using padding," in $20^{\text {th }} \mathrm{An}$ nual International Conference on High Performance Computing, Dec. 2013, pp. 425-432.

[48] M. Kistler, M. Perrone, and F. Petrini, "Cell multiprocessor communication network: Built for speed," IEEE Micro, vol. 26, no. 3, pp. 10-23, May 2006.

[49] J. Wang, J. Sohl, A. Karlsson, and D. Liu, “An efficient streaming star network for multi-core parallel DSP processor," in 2nd International Conference on Networking and Computing, Nov. 2011, pp. 332336.

[50] J. Wang, A. Karlsson, J. Sohl, M. Pettersson, and D. Liu, “A multilevel arbitration and topology free streaming network for chip multiprocessor," in $9^{\text {th }}$ IEEE International Conference on ASIC, Oct. 2011, pp. 153-158.

[51] IBM Systems and Technology Group, "Cell Broadband Engine architecture. version 1.02," Tech. Rep., 2007.

[52] Intel, "Reference manual for Intel math kernel library 11.3," Tech. Rep., 2011.

[53] Texas Instruments. DSPLIB 3.0.0.8 for C66x. http://software-dl.ti.com/sdoemb/sdoemb_public_sw/dsplib/ 3_0_0_8/index_FDS.html

[54] Y. Tang, Y. Wang, J.-G. Chung, S. Song, and M. Lim, "High-speed assembly FFT implementation with memory reference reduction on DSP processors," in Proceedings of the $11^{\text {th }}$ IEEE International Conference on Electronics, Circuits and Systems, 2004, pp. 547-550.

[55] P. A. Karlström, “NoGAP: Novel generator of accelerators and processors," Ph.D. dissertation, Linköping University, Sweden, 2010. 
[56] S. Pees, A. Hoffmann, V. Zivojnovic, and H. Meyr, "LISA-machine description language for cycle-accurate models of programmable DSP architectures," in Proceedings Design Automation Conference, 1999, pp. 933-938.

[57] "Synopsys ASIP Designer." http://www.synopsys.com

[58] Texas Instruments, “TMS320C64x+ DSP little-endian DSP library programmer's reference," Tech. Rep., 2008.

[59] R. Damodaran, T. Anderson et al., "A 1.25GHz 0.8W C66x DSP core in 40nm CMOS," in $25^{\text {th }}$ International Conference on VLSI Design, Jan. 2012, pp. 286-291.

[60] D. Liu, A. Nilsson, E. Tell, D. Wu, and J. Eilert, "Bridging dream and reality: Programmable baseband processors for softwaredefined radio," IEEE Communications Magazine, vol. 47, no. 9, pp. 134-140, Sep. 2009.

[61] M. Woh, Y. Lin et al., "From SODA to Scotch: The evolution of a wireless baseband processor," in $41^{\text {st }}$ Annual IEEE/ACM International Symposium on Microarchitecture, Lake Como, Italy, Nov. 2008, pp. 152-163.

[62] B. Gedik, R. R. Bordawekar, and P. S. Yu, "CellSort: High performance sorting on the Cell processor," in Proceedings of the $33^{\text {rd }}$ International Conference on Very Large Data Bases, Vienna, Austria, 2007, pp. 1286-1297.

[63] Freescale Semiconductor, "AltiVec technology programming interface manual," Tech. Rep., 1999.

[64] "F\# Programming Language," http:/ /www.fsharp.org.

[65] Haskell programming language. http://www.haskell.org

[66] J. W. Cooley and J. W. Tukey, "An algorithm for the machine calculation of complex Fourier series," Mathematics of Computation, vol. 19, no. 90, pp. 297-301, May 1965. 
[67] S. S. Muchnick, Advanced Compiler Design and Implementation. San Francisco, CA, USA: Morgan Kaufmann Publishers Inc., 1997.

[68] Texas Instruments, “TMS320C6000 programmer's guide," Tech. Rep., 2000.

[69] D. Talla and L. K. John, "MediaBreeze: A decoupled architecture for accelerating multimedia applications," ACM SIGARCH Computer Architecture News, vol. 29, no. 5, pp. 62-67, Dec. 2001.

[70] M. I. Soliman and A. F. Al-Junaid, "Codevelopment of multi-level ISA and hardware for an efficient matrix processor," in International Conference on Computer Engineering \& Systems, Dec. 2009, pp. 211-217.

[71] A. Pedram, A. Gerstlauer, and R. A. van de Geijn, "A highperformance, low-power linear algebra core," in $22^{\text {nd }}$ IEEE International Conference on Application-specific Systems, Architectures and Processors, Sep. 2011, pp. 35-42.

[72] K. Mizumoto, T. Tanizaki et al., "A multi matrix-processor core architecture for real-time image processing SoC," in IEEE Asian SolidState Circuits Conference, Nov. 2007, pp. 180-183.

[73] NVIDIA, “CUDA C programming guide. version 7.5," Tech. Rep., 2015.

[74] M. Gossel, B. Rebel, and R. Creutzburg, Memory Architecture and Parallel Access. New York, NY, USA: Elsevier Science Inc., 1994.

[75] A. Karlsson, J. Sohl, and D. Liu, "Software-based QPP interleaving for baseband DSPs with LUT-accelerated addressing," in IEEE International Conference on Digital Signal Processing, Jul. 2015.

[76] D. H. Lawrie and C. R. Vora, "The prime memory system for array access," IEEE Transactions on Computers, vol. C-31, no. 5, pp. 435442, May 1982. 
[77] W. B. Pennebaker and J. L. Mitchell, JPEG Still Image Data Compression Standard, 1st ed. Norwell, MA, USA: Kluwer Academic Publishers, 1992.

[78] "ITU-T Recommendation H.264 : Advanced video coding for generic audiovisual services," International Telecommunications Union, Nov. 2007. http://www.itu.int/rec/T-REC-H. 264-200711-I/en

[79] A. Tarable, S. Benedetto, and G. Montorsi, "Mapping interleaving laws to parallel turbo and LDPC decoder architectures," IEEE Transactions on Information Theory, vol. 50, no. 9, pp. 2002-2009, Sep. 2004.

[80] A. Karlsson, "Algorithm adaptation and optimization of a novel DSP vector co-processor," Master's thesis, Linköping University, Computer Engineering, 2010.

[81] K. D. Cooper, L. T. Simpson, and C. A. Vick, "Operator strength reduction," ACM Transactions on Programming Languages and Systems, vol. 23, no. 5, pp. 603-625, Sep. 2001.

[82] J.-L. Cruz, A. González, M. Valero, and N. P. Topham, "Multiplebanked register file architectures," in Proceedings of the $27^{\text {th }}$ Annual International Symposium on Computer Architecture, 2000, pp. 316325.

[83] R. Shioya, K. Horio, M. Goshima, and S. Sakai, "Register cache system not for latency reduction purpose," in $43^{\text {rd }}$ Annual IEEE/ACM International Symposium on Microarchitecture, Dec. 2010, pp. 301312.

[84] L. Huang, L. Shen, Z. Wang, W. Shi, N. Xiao, and S. Ma, "SIF: Overcoming the limitations of SIMD devices via implicit permutation," in 16th International Symposium on High-Performance Computer Architecture, Jan. 2010, pp. 1-12. 
[85] G. Ren, P. Wu, and D. Padua, "Optimizing data permutations for SIMD devices," ACM SIGPLAN Notices, vol. 41, no. 6, pp. 118-131, Jun. 2006.

[86] M. Püschel, P. A. Milder, and J. C. Hoe, "Permuting streaming data using RAMs," Journal of the ACM, vol. 56, no. 2, pp. 1-34, Apr. 2009.

[87] R. Chen and V. K. Prasanna, "Automatic generation of high throughput energy efficient streaming architectures for arbitrary fixed permutations," in $25^{\text {th }}$ International Conference on Field Programmable Logic and Applications, Sep. 2015, pp. 1-8.

[88] G. Chen and M. Kandemir, "Optimizing address code generation for array-intensive DSP applications," in International Symposium on Code Generation and Optimization, Los Alamitos, CA, USA, 2005, pp. 141-152.

[89] P. Hulina, L. Coraor, L. Kurian, and E. John, “Design and VLSI implementation of an address generation coprocessor," IEEE Proceedings - Computers and Digital Techniques, vol. 142, no. 2, pp. 145-151, Mar. 1995.

[90] Intel, "Intel 64 and IA-32 architectures software developer's manual - volume 1: Basic architecture," Tech. Rep., 2015.

[91] J. Kin, M. Gupta, and W. Mangione-Smith, “The filter cache: an energy efficient memory structure," in Proceedings of $30^{\text {th }}$ Annual International Symposium on Microarchitecture, Dec. 1997, pp. 184-193.

[92] W. Tang, R. Gupta, and A. Nicolau, "Power savings in embedded processors through decode filter cache," in Proceedings Design, Automation and Test in Europe Conference and Exhibition, 2002, pp. 443448.

[93] L. H. Lee, B. Moyer, and J. Arends, "Instruction fetch energy reduction using loop caches for embedded applications with small tight loops," in Proceedings International Symposium on Low Power Electronics and Design, Aug. 1999, pp. 267-269. 
[94] A. J. Smith, "Cache memories," ACM Comput. Surv., vol. 14, no. 3, pp. 473-530, Sep. 1982.

[95] D. Grund and J. Reineke, "Estimating the performance of cache replacement policies," in $6^{\text {th }}$ ACM/IEEE International Conference on Formal Methods and Models for Co-Design, Jun. 2008, pp. 101-112.

[96] A. S. Nery, N. Nedjah, F. M. G. Franca, L. Jozwiak, and H. Corporaal, "Automatic complex instruction identification for efficient application mapping onto ASIPs," in IEEE $5^{\text {th }}$ Latin American Symposium on Circuits and Systems, Feb. 2014.

[97] K. E. Batcher, "Sorting networks and their applications," in Proceedings of the Spring Joint Computer Conference, Atlantic City, New Jersey, Apr. 1968, pp. 307-314.

[98] J. Einemo and M. Lundqvist, "A selection of H.264 encoder components implemented and benchmarked on a multi-core DSP processor," Master's thesis, Linköping University, Computer Engineering, 2010.

[99] “IEEE standard for floating-point arithmetic," pp. 1-70, Aug. 2008.

[100] L. A. Wolsey, Integer Programming. Wiley, 1998.

[101] C. Galuzzi, E. M. Panainte, Y. Yankova, K. Bertels, and S. Vassiliadis, "Automatic selection of application-specific instruction-set extensions," in Proceedings of the $4^{\text {th }}$ International Conference on Hardware/Software Codesign and System Synthesis, Oct. 2006, pp. 160-165.

[102] H. Choi, J.-S. Kim, C.-W. Yoon, I.-C. Park, S. H. Hwang, and C.-M. Kyung, "Synthesis of application specific instructions for embedded DSP software," IEEE Transactions on Computers, vol. 48, no. 6, pp. 603-614, Jun. 1999.

[103] N. Clark, M. Kudlur, H. Park, S. Mahlke, and K. Flautner, "Application-specific processing on a general-purpose core via transparent instruction set customization," in International Symposium on Microarchitecture, Dec. 2004, pp. 30-40. 
[104] A. Alomary, T. Nakata, Y. Honma, M. Imai, and N. Hikichi, "An ASIP instruction set optimization algorithm with functional module sharing constraint," in Proceedings of International Conference on Computer Aided Design, Nov. 1993, pp. 526-532.

[105] T. C. Wilson, M. K. Garg, R. Deadman, B. Halley, and D. K. Banerji, "MinMux: a new approach for global minimization of multiplexers in interconnect synthesis," in Proceedings 3rd Great Lakes Symposium on VLSI-Design Automation of High Performance VLSI Systems, Mar. 1993, pp. 132-138.

[106] Gurobi optimizer 6.00. http://www.gurobi.com/

[107] G. Estrin, “Organization of computer systems: The fixed plus variable structure computer," in Papers Presented at the Western Joint IRE-AIEE-ACM Computer Conference, San Francisco, California, May 1960, pp. 33-40.

[108] M. A. Shami and A. Hemani, "Classification of massively parallel computer architectures," in IEEE $26^{\text {th }}$ International Parallel and Distributed Processing Symposium Workshops \& PhD Forum, May 2012, pp. 344-351.

[109] M. Woh, S. Seo, S. Mahlke, T. Mudge, C. Chakrabarti, and K. Flautner, "AnySP: Anytime anywhere anyway signal processing," in Proceedings of the $36^{\text {th }}$ Annual International Symposium on Computer Architecture, Austin, TX, USA, 2009, pp. 128-139.

[110] J. Meng, J. W. Sheaffer, and K. Skadron, "Robust SIMD: Dynamically adapted SIMD width and multi-threading depth," in IEEE $26^{\text {th }}$ International Parallel and Distributed Processing Symposium, May 2012, pp. 107-118.

[111] L. Lamport, "How to make a multiprocessor computer that correctly executes multiprocess programs," IEEE Transactions on Computers, vol. 28, no. 9, pp. 690-691, Sep. 1979. 
[112] H.-J. Cheng, Y.-S. Hwang, R.-G. Chang, and C.-W. Chen, “Trading conditional execution for more registers on ARM processors," in IEEE/IFIP International Conference on Embedded and Ubiquitous Computing, Dec. 2010, pp. 53-59.

[113] S. A. Mahlke, R. E. Hank, J. E. McCormick, D. I. August, and W.M. W. Hwu, "A comparison of full and partial predicated execution support for ILP processors," in Proceedings of the $22^{\text {nd }} A n$ nual International Symposium on Computer Architecture, Jun. 1995, pp. 138-149.

[114] H. Kim, O. Mutlu, J. Stark, and Y. N. Patt, “Wish branches: Combining conditional branching and predication for adaptive predicated execution," in $38^{\text {th }}$ Annual IEEE/ACM International Symposium on Microarchitecture, Barcelona, Spain, Nov. 2005, pp. 43-54.

[115] R. M. Russell, "The CRAY-1 computer system," Communications of the ACM, vol. 21, no. 1, pp. 63-72, Jan. 1978.

[116] J. C. Dehnert, P. Y.-T. Hsu, and J. P. Bratt, “Overlapped loop support in the Cydra 5," ACM SIGARCH Computer Architecture News, vol. 17, no. 2, pp. 26-38, Apr. 1989.

[117] R. A. Ravindran, R. M. Senger et al., "Increasing the number of effective registers in a low-power processor using a windowed register file," in Proceedings of the International Conference on Compilers, Architecture and Synthesis for Embedded Systems, San Jose, California, USA, 2003, pp. 125-136.

[118] J. Park, S.-B. Park, J. D. Balfour, D. Black-Schaffer, C. Kozyrakis, and W. J. Dally, "Register pointer architecture for efficient embedded processors," in Design, Automation \& Test in Europe Conference E Exhibition, Apr. 2007, pp. 1-6.

[119] G. H. Golub and C. F. Van Loan, Matrix Computations, 3rd ed. Baltimore, MD, USA: Johns Hopkins University Press, 1996. 
[120] B. Bhattacharya and S. S. Bhattacharyya, "Parameterized dataflow modeling for DSP systems," IEEE Transactions on Signal Processing, vol. 49, no. 10, pp. 2408-2421, Oct. 2001.

[121] E. A. Lee and T. M. Parks, “Dataflow process networks," Proceedings of the IEEE, vol. 83, no. 5, pp. 773-801, May 1995.

[122] F. Franchetti and M. Püschel, "A SIMD vectorizing compiler for digital signal processing algorithms," in Proceedings $16^{\text {th }}$ International Parallel and Distributed Processing Symposium, Apr. 2002.

[123] C. Kozyrakis, D. Judd, J. Gebis, S. Williams, D. Patterson, and K. Yelick, "Hardware/compiler codevelopment for an embedded media processor," Proceedings of the IEEE, vol. 89, no. 11, pp. 16941709, Nov. 2001.

[124] A. V. Aho, R. Sethi, and J. D. Ullman, Compilers: Principles, Techniques, and Tools. Boston, MA, USA: Addison-Wesley Longman Publishing Co., Inc., 1986.

[125] M. A. Arslan and K. Kuchcinski, "Instruction selection and scheduling for DSP kernels on custom architectures," in Euromicro Conference on Digital System Design, Sep. 2013, pp. 821-828.

[126] M. A. Arslan, F. Gruian, K. Kuchcinski, and A. Karlsson, "Code generation for a SIMD architecture with custom memory organization," Rennes, France, 2016.

[127] I. Foster, Designing and Building Parallel Programs. Addison-Wesley Reading, 1995, vol. 95.

[128] OpenMP. http://www.openmp.org/

[129] Khronos. (2013) Open CL - the open standard for parallel programming of heterogeneous systems. http://www.khronos. org/opencl/

[130] G. Jo, W. J. Jeon, W. Jung, G. Taft, and J. Lee, “OpenCL framework for ARM processors with NEON support," in Proceedings of 
the Workshop on Programming Models for SIMD/Vector Processing, Orlando, Florida, USA, 2014, pp. 33-40.

[131] L. G. Valiant, "A bridging model for parallel computation," Communications of the ACM, vol. 33, no. 8, pp. 103-111, Aug. 1990.

[132] D. Johansson, M. Eriksson, and C. Kessler, "Bulk-synchronous parallel computing on the CELL processor," PARS, vol. 7, p. 21, 2007.

[133] OpenACC-Standard.org, "The OpenACC application programming interface," Tech. Rep., 2015.

[134] W. Gropp, E. Lusk, and A. Skjellum, Using MPI: Portable Parallel Programming with the Message-passing Interface, 2nd ed. Cambridge, MA, USA: MIT Press, 1999.

[135] M. Ohara, H. Inoue, Y. Sohda, H. Komatsu, and T. Nakatani, “MPI microtask for programming the Cell Broadband Engine ${ }^{\mathrm{TM}}$ processor," IBM Systems Journal, vol. 45, no. 1, pp. 85-102, 2006.

[136] M. Abadi, A. Agarwal et al., "TensorFlow: Large-scale machine learning on heterogeneous distributed systems," CoRR, 2016.

[137] J. Eker and J. W. Janneck, "CAL language report specification of the CAL actor language," EECS Department, University of California, Berkeley, Tech. Rep. UCB/ERL M03/48, 2003.

[138] E. Gebrewahid, M. A. Arslan, A. Karlsson, and Z. Ul-Abdin, "Support for data parallelism in the CAL actor language," in Proceedings of the $3^{\text {rd }}$ Workshop on Programming Models for SIMD/Vector Processing, Barcelona, Spain, 2016, pp. 21-28.

[139] M. Cole, Algorithmic Skeletons: Structured Management of Parallel Computation. Cambridge, MA, USA: MIT Press, 1991.

[140] M. Ålind, M. V. Eriksson, and C. W. Kessler, “BlockLib: A skeleton library for Cell broadband engine," in Proceedings of the $1^{\text {st }}$ International Workshop on Multicore Software Engineering, Leipzig, Germany, 2008. 
[141] U. Dastgeer, J. Enmyren, and C. W. Kessler, “Auto-tuning SkePU: A multi-backend skeleton programming framework for multi-GPU systems," in Proceedings of the $4^{\text {th }}$ International Workshop on Multicore Software Engineering, Waikiki, Honolulu, HI, USA, 2011, pp. 25-32.

[142] L. Li and C. W. Kessler, "MeterPU: A generic measurement abstraction API enabling energy-tuned skeleton backend selection," in IEEE Trustcom/BigDataSE/ISPA, Aug. 2015.

[143] Y. Zhang, W. Zhou, Z. Liu, S. Wang, and D. Liu, "A high definition motion JPEG encoder based on ePUMA platform," Procedia Engineering, vol. 29, pp. 2371-2375, 2012.

[144] Texas Instruments. MATHLIB 3.0.0.0 for c66x. http://software-dl.ti.com/sdoemb/sdoemb_public_sw / mathlib/3_0_0_0/index_FDS.html

[145] Texas Instruments. IMGLIB 3.0.1.0 for C66x. http://software-dl.ti.com/sdoemb/sdoemb_public_sw/imglib/ 3_0_1_0/index_FDS.html

[146] S. Aylor, "StarCore high-performance DSP product roadmap," Tech. Rep., 2010.

[147] F. Franchetti and M. Püschel, "Generating SIMD vectorized permutations," in International Conference on Compiler Construction, vol. 4959, 2008, pp. 116-131.

[148] J. Sun and O. Takeshita, "Interleavers for turbo codes using permutation polynomials over integer rings," IEEE Transactions on Information Theory, vol. 51, no. 1, pp. 101-119, Jan. 2005.

[149] Analog Devices, "TigerSHARC embedded processor - ADSPTS201S," Tech. Rep., 2006.

[150] B. Beheshti, “On performance of LTE UE DFT and FFT implementations in flexible software based baseband processors," in IEEE Long Island Systems, Applications and Technology Conference, May 2009, pp. 1-4. 
[151] Freescale Semiconductor, "MSC8156EVM Kernels Starting Guide," http://www.freescale.com/.

[152] S. Winograd, "On computing the discrete Fourier transform," Mathematics of Computation, vol. 32, no. 141, pp. 175-199, 1978.

[153] F. Qureshi, M. Garrido, and O. Gustafsson, “Unified architecture for 2, 3, 4, 5, and 7-point DFTs based on Winograd Fourier transform algorithm," Electronics Letters, vol. 49, no. 5, pp. 348-349, Feb. 2013.

[154] G. Wang, B. Yin, I. Cho, J. R. Cavallaro, S. Bhattacharyya, and J. Takala, "Efficient architecture mapping of FFT/IFFT for cognitive radio networks," in IEEE International Conference on Acoustics, Speech and Signal Processing, May 2014, pp. 3933-3937.

[155] A. Karlsson, J. Sohl, and D. Liu, "Cost-efficient mapping of 3- and 5-point DFTs to general baseband processors," in IEEE International Conference on Digital Signal Processing, Jul. 2015.

[156] Freescale Semiconductor, "Software optimization of DFTs and IDFTs using the StarCore SC3850 DSP core," Tech. Rep., 2010.

[157] NVIDIA, “GeForce GTX 580 specifications," http:/ / www.geforce. com/hardware/desktop-gpus/geforce-gtx-580/specifications.

[158] J. Gómez-Luna, J. M. González-Linares, J. I. Benavides, and N. Guil, "An optimized approach to histogram computation on GPU," Machine Vision and Applications, vol. 24, no. 5, pp. 899-908, Jul. 2013.

[159] N. Satish, C. Kim et al., "Fast sort on CPUs and GPUs: A case for bandwidth oblivious SIMD sort," in Proceedings of the ACM SIG$M O D$ International Conference on Management of Data, Indianapolis, Indiana, USA, 2010, pp. 351-362.

[160] S. A. Fahmy, P. Y. K. Cheung, and W. Luk, "High-throughput onedimensional median and weighted median filters on FPGA," IET Computers $\mathcal{E}$ Digital Techniques, vol. 3, no. 4, pp. 384-394, 2009. 
[161] P. Kolte, R. Smith, and W. Su, "A fast median filter using AltiVec," in Proceedings of the IEEE International Conference on Computer Design, Washington, DC, USA, 1999, pp. 384-391.

[162] A. Stefan and S. Zoican, “The evaluation of image noise reduction filter implementation with the Blackfin microcomputer," in $8^{\text {th }} I n$ ternational Conference on Communications, Jun. 2010, pp. 181-184.

[163] A. Karlsson, J. Sohl, and D. Liu, "ePUMA: A processor architecture for future DSP," in IEEE International Conference on Digital Signal Processing, Jul. 2015.

[164] C. E. Kozyrakis and D. A. Patterson, "Vector vs. superscalar and VLIW architectures for embedded multimedia benchmarks," in Proceedings of the $35^{\text {th }}$ Annual IEEE/ACM International Symposium on Microarchitecture, 2002, pp. 283-293.

[165] A. Karlsson, J. Sohl, W. J., and D. Liu, "ePUMA: A unique memory access based parallel DSP processor for SDR and CR," in IEEE Global Conference on Signal and Information Processing, Dec. 2013, pp. 1234-1237.

[166] R. Hultén, C. W. Kessler, and J. Keller, “Optimized on-chippipelined mergesort on the Cell/B.E." in Euro-Par - Parallel Processing, 2010, vol. 6272, pp. 187-198.

[167] N. Satish, C. Kim et al., "Fast sort on CPUs, GPUs and Intel MIC architectures," Intel Labs, Technical Report, 2010.

[168] H. Inoue, T. Moriyama, H. Komatsu, and T. Nakatani, “AA-sort: A new parallel sorting algorithm for multi-core SIMD processors," in Proceedings of the $16^{\text {th }}$ International Conference on Parallel Architecture and Compilation Techniques, Washington, DC, USA, Sep. 2007, pp. 189-198.

[169] J. Chhugani, A. D. Nguyen et al., "Efficient implementation of sorting on multi-core SIMD CPU architecture," Proc. VLDB Endow., vol. 1, no. 2, pp. 1313-1324, Aug. 2008. 
[170] Intel Corporation. Intel ARK. http://ark.intel.com/

[171] NVIDIA, “GeForce GTX Titan specifications," http://www. geforce.com/hardware/desktop-gpus/geforce-gtx-titan/ specifications.

[172] T. Xiaochen, K. Rocki, and R. Suda, "Register level sort algorithm on multi-core SIMD processors," in Proceedings of the $3^{\text {rd }}$ Workshop on Irregular Applications Architectures and Algorithms, Denver, Colorado, 2013, pp. 91-98.

[173] P. Xiaoyi, "Benchmark of MPEG-2 video decoding on ePUMA multi-core DSP processor," Master's thesis, Linköping University, Computer Engineering, 2011.

[174] I. Ragnemalm and A. Karlsson, "Computing the Euclidean distance transform on the ePUMA parallel hardware," in Computer Graphics, Visualization, Computer Vision and Image Processing, 2011, pp. 228-232.

[175] I. Ragnemalm and D. Liu, "Adapting the ePUMA architecture for hand-held video games," International Journal of Computer Information Systems and Industrial Management Applications, vol. 4, pp. 153160, 2012.

[176] J. Ogniewski, A. Karlsson, and I. Ragnemalm, “Texture compression in memory and performance-constrained embedded systems," in Computer Graphics, Visualization, Computer Vision and Image Processing, 2011, pp. 19-26.

[177] J. Tolunay, "Parallel gaming related algorithms for an embedded media processor," Master's thesis, Linköping University, Information Coding, 2012. 


\section{Part VII}

\section{Appendix}





\section{Appendix A}

ePUMA hardware/software

\section{components}

The ePUMA system has been developed by several people over the course of several years. This list of hardware- and software-components tries to summarize the work and current state of the project, as well as acknowledge the main responsible/contributor of each of the components.

Hardware components:

- ePUMA system architecture (By Jian Wang) The hardware architecture of the ePUMA system.

- ePUMA memory subsystem (By Jian Wang) The ePUMA Network-on-Chip and associated memory.

- ePUMA control core architecture (By Jian Wang/Andréas Karlsson) The base architecture used for both the ePUMA master processor and ePUMA Computer Cluster Controller.

- ePUMA Compute Cluster architecture (By Andréas Karlsson) The architecture of the ePUMA compute cluster.

- ePUMA Matrix Processing Element (By Andréas Karlsson) The hardware architecture of the ePUMA Matrix Processing Element. 
Software components:

- ePUMA System Simulator (By Jian Wang)

A cycle-accurate simulator for the ePUMA system.

- ePUMA Multi-Threaded System Simulator (By Andréas Karlsson) An extension of the ePUMA System Simulator for improved simulation performance.

- ePUMA Power Simulator (By Andréas Karlsson)

An extension of the ePUMA Multi-Threaded System Simulator that tracks processor events to estimate the system energy/power consumption. The energy model is based on gate-level energy simulation.

- ePUMA Simple OS (By Jian Wang)

A light-weight operating system kernel for ePUMA, including simple task scheduling.

- ePUMA Platform Builder (By Andréas Karlsson)

A tool for generating an ePUMA system from a given specification. The tool will generate RTL code and definition files for system simulation.

- ePUMA Skeleton Library (By Andréas Karlsson)

A library of algorithmic skeletons for simple implementation of common parallel computing patterns.

- ePUMA Task Scheduler (By Joar Sohl)

A tool for multi-core task scheduling of static data flow graphs for ePUMA.

- ePUMA Linker/Loader (By Joar Sohl)

A linker/loader for the ePUMA system.

- ePUMA Build System (By Jian Wang)

A build system for ePUMA software projects, which invokes necessary tools such as compilers and assemblers, to generate a full ePUMA software image. 
- MPE Kernel Simulator (By Andréas Karlsson)

A compute kernel simulator for the ePUMA MPE.

- MPE Kernel Library (By Andréas Karlsson)

An extensive set of optimized compute kernels for the ePUMA MPE.

- MPE Macro Assembler (By Joar Sohl)

An assembler for the ePUMA MPE, which includes more advanced features such as permutation table compaction, data conflict resolving and more.

- MPE Assembly Optimizer (By Andréas Karlsson)

A tool for optimizing assembly code, such as instruction rescheduling, simple code transformations and some other optimizations. This tool is scheduled for integration with the MPE Macro Assembler and/or the eOctave compiler.

- MPE Disassembler (By Andréas Karlsson)

A disassembler for the ePUMA MPE.

- MPE Core Builder (By Andréas Karlsson)

A tool for generating the RTL code and simulator definitions for an ePUMA MPE.

- MPE Datapath Builder (By Andréas Karlsson)

A tool for generating an ePUMA MPE datapath, including instruction set selection and datapath network optimization using ILP.

- FT Compiler (By Joar Sohl)

A compiler for the prototype FT-language, for implementation of kernels for the ePUMA MPE.

- eOctave Compiler (By Andréas Karlsson)

An eOctave-compiler for the ePUMA MPE, for implementation of compute kernels. 


\section{Appendix B \\ ePUMA development timeline}

The ePUMA project has gone through several revisions and redesigns through the years. Also, the name of certain components have been changed in the redesign process. This chapter tries to summarize the different revisions. It may be helpful when reading older ePUMA material.

\section{B.1 ePUMA version 1.0}

The first generation ePUMA processor was based on the Senior master processor and eight SIMD processors, called Sleipnir. The eight SIMD processors only contained traditional SIMD instructions enhanced iteration, but it was based around the conflict-free parallel memory access concept and LUT-based addressing. The SIMD processor pipelines were statically scheduled. This platform was used during the kernel implementation of several matrix operation kernels and early baseband kernels. It was also used for the first ePUMA demo - the Motion-JPEG-based web camera.

The Senior processor, originally designed at the Computer Engineering group for teaching purposes, wasn't appropriate for use in ePUMA 
in the long run. Specifically, the compiler was immature and it was designed to be a simple DSP with local memory only. The project needed a general processor for application control, preferably also with cache support. Several problems with the Sleipnir SIMD processors had also been identified which required further improvement. It lacked some address generation support that could be of good use, but the pipeline structure sometimes prevented efficient instruction scheduling. Last but not least, we learned the hard way that programmability aspects should not be underestimated. Sleipnir was considered too hard/time-consuming to program and no compiler was ever developed.

\section{B.2 ePUMA version 1.5}

The intermediate version between the 1.0 and 2.0 releases modified the Sleipnir SIMD processor into a vector processor and increased the AGU functionality. At the same time, the Sleipnir SIMD processor was renamed the ePUMA Vector Processing Element (VPE). The Senior master processor was replaced with a cache-based processor, the ePUMA Application Processing Element (APE) and a C compiler was now in working order.

\section{B.3 ePUMA version 2.0}

ePUMA version 2.0 is the version presented in this thesis. Compared to the older 1.5 architecture, the compute cluster and compute cluster controller were introduced, which effectively made a node in the NoC a multi-core processor. In addition, the VPE was redesigned based on a compiler/hardware co-design approach. Earlier, the focus had only been on compute-performance and minimal hardware cost. The new version added some hardware to simplify some aspects of the compiler, while still keeping the high-performance profile of the processor. The compiler/hardware co-design process was highly influenced by both functional programming and numerical computing. Instead of starting from 
a hardware-view, many design choices started from some example language DSP code in our own programming language and design choices were made to minimize both compiler and hardware complexity. This resulted in the ePUMA Matrix Processing Element (MPE). While it still retained many aspects of the older VPE, many changes were done. The pipeline also got full stall logic. The first generation MPE compiler was the FT compiler and the second generation compiler was the eOctave compiler.

\section{B.4 ePUMA version 3.0}

The next generation ePUMA (in development) investigates how to bring the concepts proposed in this thesis into the shared memory programming world. Program memories throughout the architecture are replaced with caches to remove the need for explicit program code movement. At least one LVM per MPE is replaced with a cache. Still, one or multiple scratchpad LVMs per MPE are kept for high-performance predictable kernel execution. This approach may simplify the implementation of many applications, while still keeping most of the benefits of the ePUMA version 2.0 platform. This architecture is currently implemented in a simulator, but not yet in RTL. 


\section{Appendix C \\ MPE kernel library}

This appendix lists the complete list of kernels the MPE evaluation in this thesis is based on. All functions may be used stand-alone (in which case we refer to them as kernels) or as building blocks to create more complex kernels.

Most kernels accept multiple input parameters, such as the number of elements/blocks/matrices to process or instruction options such as scaling, rounding mode or whether or not to use saturation arithmetic. Most kernels exist in multiple versions, e.g. one for each reasonable data type. A few kernels exist in multiple versions due to different data distribution of input/output data. In some cases there are also multiple versions which have been optimized differently for different input data sizes.

Single element-wise operations

Basic arithmetic

Rounding

Scale (floating-point)

Logarithm

Exponentiation

Trigonometric

Reciprocals

Square-root (reciprocal)

Bitwise logical

$$
\begin{aligned}
& -x,|x|, z^{*}(\text { complex conjugate) } \\
& \operatorname{round}(x), \text { floor }(x), \operatorname{ceil}(x) \\
& x \cdot 2^{y} \\
& \log _{2}(x), \ln (x) \\
& e^{x}, 2^{x} \\
& \sin (x), \cos (x), \tan (x) \\
& \sin ^{-1}(x), \cos ^{-1}(x), \tan ^{-1}(x) \\
& 1 / x, 1 /|x|, 1 /|x|^{2} \\
& \sqrt{x}, 1 / \sqrt{x}
\end{aligned}
$$

INVERT 
Shift operations with const.

$x>>C, x<<C, x>>>C$

Random value generation

\section{Dual element-wise operations}

Basic arithmetic

$$
\begin{aligned}
& x+y, x-y, x * y, x / y \\
& |x|+|y|,|x|-|y|,|x-y| \\
& -x * y,-|x| * y \\
& \min / \max (x, y), \min / \max (|x|,|y|) \\
& a=a+b * c \\
& x \bmod L \\
& x \bmod L: 0 \leq x<2 L \\
& \text { AND/OR/XOR } \\
& x<<y, x>>y, x>>>y \\
& z=\operatorname{copysign}(x, y)
\end{aligned}
$$

Multiply and accumulate $\quad a=a+b * c$

General modulo $\quad x \bmod L$

Fast modulo

Bitwise logical

Shift operations

Copy sign (floating-point only)

\section{Single vector operations}

Sum

$$
\begin{aligned}
& \operatorname{sum}\left(\forall x_{i}\right), \operatorname{sum}\left(\forall\left|x_{i}\right|\right), \\
& \operatorname{sum}\left(\forall x_{i}^{2}\right), \operatorname{sum}\left(\forall\left|x_{i}\right|^{2}\right)
\end{aligned}
$$

Average

Minimum/maximum

$$
\min / \max \left(\forall x_{i}\right)
$$$$
\min / \max \left(\forall\left|x_{i}\right|\right), \min / \max \left(\forall\left|x_{i}\right|^{2}\right)
$$

Value search

$x_{i}=$ value?, $\min / \max \left(\forall x_{i}\right)$

$$
\begin{aligned}
& \min / \max \left(\forall\left|x_{i}\right|\right) \\
& \min / \max \left(\forall\left|x_{i}\right|^{2}\right)
\end{aligned}
$$

Sequence/string-search

Inclusive/exclusive prefix ops. $\mathrm{sum} / \mathrm{min} / \max$

\section{Dual vector operations}

Dot product

Cross product

Convolution

Auto-correlation

Correlation 
Compact

Complex radix butterfly

\section{Matrix operations}

Multiplication

Transpose

Decompositions

Determinant

Matrix inversion

\section{Pack}

Pack high/low bytes

\section{Filters}

FIR-filter

IIR-filter

Comb filter

Interpolation filter

Decimation filter

2D-convolution

\section{Transforms}

FFT/IFFT $2^{x}$-point

Mixed-radix LTE-FFT/IFFT

DCT/IDCT

2D-DCT

Wavelet transform

Walsh-Hadamard transform data + predicate

2, 3, 4, 5, 7-point

dense and sparse

normal and conjugate

Cholesky, LUD, QRD, SVD high/low(u/s16) -> packed bytes

general and symmetrical
Based on radix-2/4 butterflies

General,

8-point for MPEG/JPEG, H.264

8x8-point for MPEG/JPEG

\section{Lookup}

Parallel lookup

Histogram 


\section{Image}

Morphological dilation/erosion

Order-filtering

Saturation operations

Sobel filter

Sum of absolute difference

Color pack/unpack/convert

FAST corner detection

\section{Forward Error Correction}

General (de)interleaving

QPP (de)interleaving

Row-column interleaving

Turbo-encoding

\section{Datatype conversion}

uYY: YY-bit unsigned fixed-point

sYY: $Y Y$-bit signed fixed-point

fp32 : 32-bit floating-point general and median

threshold and clipping

$3 \times 3,5 \times 5,7 \times 7$ windows

(with pruning)

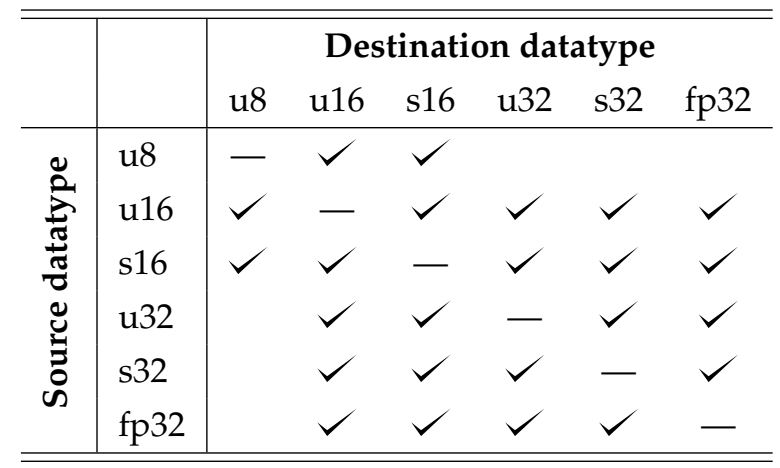

